
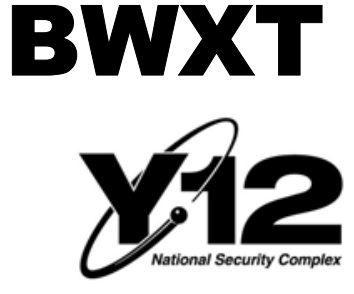

A BWXT/Bechtel Enterprise
Report No. Y/LB-16,144

Fission Multiplicity Detection with Temporal Gamma-Neutron Discrimination from Higher-Order Time Correlation Statistics

\author{
R. B. Oberer
}

Y-12

National

Security

Complex Readiness in Technical Base and Facilities

MANAGED BY BWXT Y-12, L.L.C. FOR THE UNITED STATES DEPARTMENT OF ENERGY
Material Stewardship Program

Prepared by the Y-12 National Security Complex

Oak Ridge, Tennessee 37831 managed by BWXT Y-12, L.L.C. for the

November 12, 2002

$$
\text { for the }
$$

U.S. DEPARTMENT OF ENERGY under contract DE-AC05-00OR22800 


\section{DISCLAIMER}

This report was prepared as an account of work sponsored by an agency of the United States government. Neither the United States Government nor any agency thereof, nor any of their employees, makes any warranty, express or implied, or assumes any legal liability or responsibility for the accuracy, completeness, or usefulness of any information, apparatus, product, or process disclosed, or represents that its use would not infringe privately owned rights. Reference herein to any specific commercial product, process, or service by trade name, trademark, manufacturer, or otherwise, does not necessarily constitute or imply its endorsement, recommendation, or favoring by the United States Government or any agency thereof. The views and opinions of authors expressed herein do not necessarily state or reflect those of the United States Government or any agency thereof. 


\title{
Fission Multiplicity Detection with Temporal Gamma-Neutron Discrimination from Higher-Order Time Correlation Statistics
}

\author{
A Thesis \\ Presented to \\ The Academic Faculty \\ by \\ Richard B. Oberer \\ In Partial Fulfillment \\ of the Requirements for the Degree
}

Doctor of Philosophy in the School of Mechanical Engineering 
Fission Multiplicity Detection with Temporal Gamma-Neutron Discrimination from Higher-Order Time Correlation Statistics

Approved:

C. K. Chris Wang, Committee Chair

John T. Mihalczo, Advisor

Farzad Rahnema

Weston M. Stacey, Jr.

Erik Verriest

Date Approved 


\section{ACKNOWLEDGMENTS}

Without John Mihalczo this thesis would certainly not have been possible. He and his coworkers invented the Nuclear Materials Identification System (NMIS) on which this thesis is based. This system, which grew out of the source-driven subcritical noise analysis method, is comprised of a ${ }^{252} C f$ ion-chamber source, plastic scintillating detectors and a five channel, all-two-and-three coincidence time analyzer. All of these elements are novel in NDA. He also proposed source correlation for the Random Driver (one of the Fission Multiplicity Detection (FMD) systems.[34]

Without my wife, Lisa Chiang, this thesis would also not have come about. Not only was getting the Ph.D. her idea, she joined the NMIS group two years before I did. It was from her thesis that I got the idea for mine.

C-K Chris Wang was my thesis advisor. He introduced Lisa and me to the NMIS group. One of his other students John Zino wanted to do a subcritical measurement for his thesis. He and one of Farzhad Rhanama's students Michelle Pitts had arranged to work with John Mihalczo and Tim Valentine on a subcritical measurement of reactor fuel from Missouri University Research Reactor.[50] Lisa and I went along on a field trip to ORNL for that project. Who would have guessed where that field trip would lead?

Jim Mullens, John Mattingly and Tim Valentine were the NMIS team when Lisa and I arrived at ORNL. They helped make the transition from source-driven subcritical noise analysis to NMIS at least as it currently exists. The previous version of the hardware filled a semi-trailer.[36]

John Mattingly was influential early on in the direction of both Lisa's thesis and mine. Jim Mullens had the deepest understanding of NMIS and was the most willing to share that knowledge. Jim of course deserves special recognition for goading John Mattingly. ${ }^{1}$

\footnotetext{
${ }^{1}$ See Reference $[29]$ at page iv.
} 
David Holcomb gave me my first assignment at ORNL. After two years and the money had run out, I had to give up the dream of inventing a solid-state thermal neutron detector.

Joe Williams provided the $\mathrm{Pu}-\mathrm{Be}$ source for the measurement of the correlations produced from an alpha-n reaction. That measurement was performed practically as the $\mathrm{Pu}-\mathrm{Be}$ source was being hauled away, never to be seen again. Joe's lab was a museum of ionizingradiation detection.

Sara Pozzi created the MCNP-POLIMI code which was necessary for the inelastic scattering simulation. Ficaro's KENO-NR[17], the first Monte Carlo code produced under the direction of John Mihalczo did not handle gamma rays at all. The second attempt at a Monte Carlo code for NMIS was MCNP-DSP by Tim Valentine. Although it tracked gamma rays, it did not preserve the multiplicity of gamma rays produced by the inelastic scatter of neutrons. It could therefore not predict the detector-detector correlations produced by that interaction.

The NMIS lab, in room D21 in Building 3500 at ORNL, was located next to Joe Williams' lab and across the hall from John Mihalczo's office. Tim Valentine had helped Sara Pozzi and Lisa set up a time-to-amplitude converter (TAC) with two plastic scintillating detectors and a small ${ }^{252} \mathrm{Cf}$ pellet source. One detector went to the start and the other went to the stop of the TAC. It was puzzling to us how the gamma rays were detected by the stop detector before the neutrons were detected in the start detector yet the arrival time at the TAC was reversed with a difference in cable length. The discussions about that simple experiment among the three of us were the first steps on the journey toward three Ph.D. theses. 


\section{TABLE OF CONTENTS}

ACKNOWLEDGMENTS

LIST OF TABLES viii

LIST OF FIGURES ix

LIST OF SYMBOLS - xii

SUMMARY

\section{CHAPTERS}

I INTRODUCTION 1

II CURRENT AND PAST PRACTICES OF PASSIVE NEUTRON AND FISSION MULTIPLICITY COUNTING TECHNIQUES FOR NDA 4

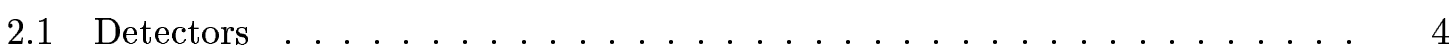

2.2 Thermal well and shift register techniques . . . . . . . . . . . 5

2.3 Time Interval Analysis $(\mathrm{TIA}) \ldots \ldots \ldots \ldots \ldots$

2.4 Fission Multiplicity Detectors (FMD) . . . . . . . . . . . . . 14

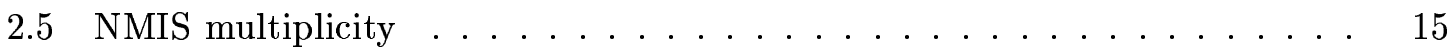

III TIME ANALYSIS AND DEFINITION OF TIME CORRELATION 17

3.1 NMIS Correlations - simple examples . . . . . . . . . . . . . . . 18

3.2 Definition of the correlation function . . . . . . . . . . . . . 21

3.3 Correlation function in a thermal neutron system . . . . . . . . . . . . 22

3.4 Uncorrelated background and accidental correlations . . . . . . . . . . . 23

3.5 Correlation function in a fast neutron system . . . . . . . . . . 25

3.5.1 Detector efficiency in a fast neutron detector $\ldots \ldots \ldots 27$ 
3.6 Error Analysis . . . . . . . . . . . . . . . . . . . 28

IV ANALYTICAL EXPRESSIONS AND TEMPORAL SEPARATION OF NEUTRON AND GAMMA COINCIDENCES FROM FISSION AND $\begin{array}{ll}\text { OTHER SOURCES } & 31\end{array}$

4.1 Source and detector correlations from a point fission source . . . . . . . . 32

4.2 Ratios of source-detector correlations . . . . . . . . . . . . . . . . . 42

4.3 Ratios of detector-detector correlations in passive measurement . . . . . . . 44

4.4 Multiplicity data . . . . . . . . . . . . . . . . 46

4.4 .1 Neutron multiplicity . . . . . . . . . . . . . . . 46

4.4 .2 Gamma-ray multiplicity . . . . . . . . . . . . . . . 46

4.5 Factors affecting time distribution of correlations . . . . . . . . . . . 50

4.5.1 Spatial distribution of material . . . . . . . . . . . 50

4.5.2 Scattering in matrix and surrounding materials . . . . . . . . . 51

4.5.3 Neutron multiplication . . . . . . . . . . . . . . . 51

4.6 Correlated detections from non-fission reactions . . . . . . . . . . . 51

$4.6 .1 \quad$ Nuclear decay . . . . . . . . . . . . . . . . . . 52

$4.6 .2 \quad$ Elastic scattering . . . . . . . . . . . . . . . 54

$4.6 .3 \quad$ Inelastic scattering . . . . . . . . . . . . . . . . . 54

4.6 .4 Alpha-n $(\alpha, n)$ reactions $\ldots \ldots \ldots \ldots \ldots \ldots$

4.6 .5 Combined reactions $\ldots \ldots \ldots \ldots \ldots$

V NEUTRON MULTIPLICATION

5.1 The time evolution of the multiplication process $\ldots \ldots \ldots 2$

5.2 Neutron singles, doubles and triples $\ldots \ldots \ldots \ldots$. . . . . . . 65

5.3 Gamma singles, doubles and triples . . . . . . . . . . . . . . . 69

5.4 Gamma-neutron combinations . . . . . . . . . . . . . . . 71 
$\begin{array}{lll}\text { VII EXPERIMENTAL DESCRIPTION } & 80\end{array}$

7.1 Functional block diagram . . . . . . . . . . . . . . . . . . 80

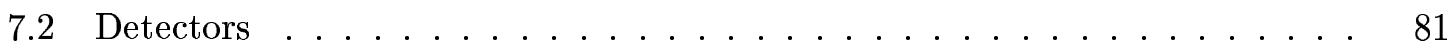

$7.3 \quad{ }^{252} C f$ ionization chamber source $\ldots \ldots \ldots \ldots \ldots$

VIII EXPERIMENTAL RESULTS

8.1 Source correlations . . . . . . . . . . . . . . . . . . . 87

8.2 Passive detector correlations . . . . . . . . . . . . . . . . 89

8.2 .1 Time dependent $\operatorname{ratios} \ldots \ldots \ldots \ldots . \ldots \ldots$

8.3 Comparison between 3 -inch and 4 -inch detectors $\ldots \ldots \ldots$. . . . . . 94

8.4 Determination of mass from gamma-neutron-neutron triplets . . . . . . . 101

$\begin{array}{ll}\text { IX CONCLUSIONS } & 106\end{array}$

APPENDIX A - FACTORIAL MOMENTS FROM PROBABILITY GEN$\begin{array}{ll}\text { ERATING FUNCTIONS } & 109\end{array}$

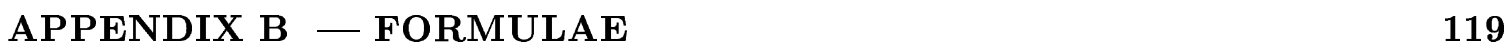

REFERENCES

$\begin{array}{ll}\text { VITA } & 127\end{array}$ 


\section{LIST OF TABLES}

$1 \quad$ Spontaneous fission data $\ldots \ldots \ldots \ldots \ldots \ldots$

$2 \quad$ Correlation data accumulated by NMIS . . . . . . . . . . . . 18

$3 \quad$ Temporal bounds for $C_{123}\left(\tau_{12}, \tau_{13}\right)$ and $C_{23}\left(\tau_{23}\right) \ldots \ldots \ldots$

$4 \quad$ Temporal bounds for $C_{234}\left(\tau_{23}, \tau_{24}\right) \ldots \ldots \ldots \ldots \ldots \ldots$

5 Neutron multiplicities and factorial moments . . . . . . . . . . . 47

6 Gamma-ray multiplicities and factorial moments . . . . . . . . . . . . 49

$7 \quad$ Multiplicity moments for time correlations . . . . . . . . . . . . . 53

8 Isotopic composition of the $\mathrm{Pu}-\mathrm{Be}$ source $\ldots \ldots \ldots \ldots$

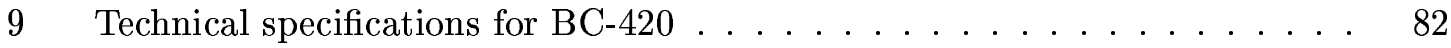

10 Parameters for 4 -inch detectors . . . . . . . . . . . . . . . . 83

11 Spontaneous Decay of ${ }^{252} C f \ldots \ldots \ldots \ldots \ldots$. . . . . . . . 84

12 Correlation data for 3 -inch and 4 -inch detectors . . . . . . . . . . . 86

13 Passive ratios for 3 -inch detectors . . . . . . . . . . . . . . . . . 91

14 Passive ratios for 4 -inch detectors . . . . . . . . . . . . . . . . 91

15 Gamma-ray detection efficiency for detector $k \ldots \ldots \ldots$. . . . . . 92

16 Neutron detection efficiency for detector $k \ldots \ldots \ldots \ldots$. . . . . . . 92

17 Ratio of $\left(C_{g n}\right)^{2}$ with $C_{g n \mid g}$ and $C_{g n \mid n} \ldots \ldots \ldots \ldots \ldots$

18 Comparison of 3 -inch and 4 -inch detector efficiencies. . . . . . . . . . 100

19 Determination of the number of spontaneous fissions . . . . . . . . . . . . 104

20 Summary of the ${ }^{252} C f$ mass determination . . . . . . . . . . . . . 107 


\section{LIST OF FIGURES}

1 Shift register functional diagram. . . . . . . . . . . . . . . . 7

2 Single block correlations. . . . . . . . . . . . . . . . . . . . 19

$3 \quad$ Independent correlation. . . . . . . . . . . . . . . . . . . . . . 20

4 Dependent correlation. . . . . . . . . . . . . . . . . . . 21

$5 \quad$ Window bias. . . . . . . . . . . . . . . . . . 21

6 Source-detector correlations for a thermal detector rate of $\frac{1}{100 \Delta t}$. . . . . 24

$7 \quad$ Source-detector correlations for a thermal detector rate of $\frac{1}{10 \Delta t} \ldots \ldots \ldots 25$

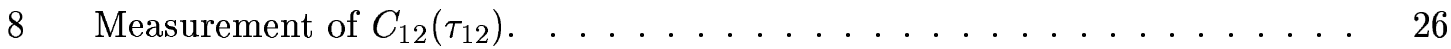

$9 \quad$ Intrinsic neutron detection efficiency. . . . . . . . . . . . . . . 28

10 Measurement of $C_{12}\left(\tau_{12}\right) \ldots \ldots \ldots \ldots \ldots \ldots \ldots$

11 Measurement of $C_{123}\left(\tau_{12}, \tau_{13}\right) \ldots \ldots \ldots \ldots \ldots \ldots \ldots \ldots \ldots$

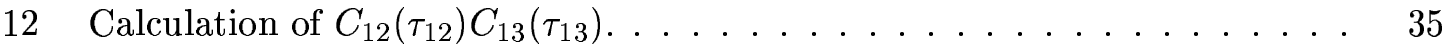

13 Measurement of $C_{23}\left(\tau_{23}\right) \ldots \ldots \ldots \ldots \ldots \ldots \ldots$

14 Measurement of $C_{234}\left(\tau_{23}, \tau_{24}\right) \ldots \ldots \ldots \ldots \ldots \ldots \ldots$

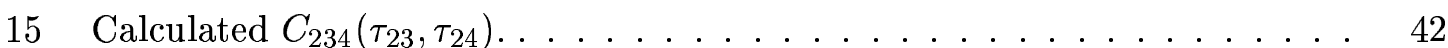

16 Neutron multiplicities for ${ }^{252} C f$ and ${ }^{240} \mathrm{Pu} . \ldots \ldots \ldots . \ldots . \ldots 4$

17 Gamma ray multiplicities for ${ }^{252} C f$ and ${ }^{240} \mathrm{Pu} \ldots \ldots \ldots \ldots$

18 Cross correlations from inelastic scattering. . . . . . . . . . . . 56

19 Experimental setup for the $\mathrm{Pu}$-Be experiment. . . . . . . . . . . . 57

20 Cross correlations from a $\mathrm{Pu}$-Be source. . . . . . . . . . . . . . . 59

21 Fission followed by an inelastic scatter of a neutron. . . . . . . . . . . 59

22 Probability of induced fission. . . . . . . . . . . . . . . 63

23 Theoretical $p_{12}\left(\tau_{12}\right)$ convolved with exponential fission. . . . . . . . . 65

24 Comparison of theoretical $p_{23}\left(\tau_{23}\right)$ with and without multiplication. . . . 66

25 Theoretical $p_{234}\left(\tau_{23}, \tau_{24}\right)$ without multiplication. . . . . . . . . . 67

26 Theoretical $p_{234}\left(\tau_{23}, \tau_{24}\right)$ with multiplication. . . . . . . . . . . 67 
27 Neutron singles. . . . . . . . . . . . . . . . . . . . . . 68

28 Neutron doubles. . . . . . . . . . . . . . . . . . 68

29 Neutron Triples. . . . . . . . . . . . . . . . . . . . . . 68

30 Gamma singles. . . . . . . . . . . . . . . . . . . . . 69

31 Gamma pairs. . . . . . . . . . . . . . . . . . 70

32 Gamma triples. . . . . . . . . . . . . . . . . . 70

33 Gamma-neutron detection pairs. . . . . . . . . . . . . . . . 71

34 Gamma-gamma-neutron detection triples. . . . . . . . . . . . . . . . 71

35 Gamma-neutron-neutron detection triples. . . . . . . . . . . . . . . 72

36 Probability of dead-time loss. . . . . . . . . . . . . . . . . 74

37 Experimental setup. . . . . . . . . . . . . . . . . . . 81

38 Block diagram of NMIS. . . . . . . . . . . . . . . . . 82

39 Schematic of ${ }^{252} C f$ ionization chamber. . . . . . . . . . . . . 84

40 Experimental source-detector correlations $C_{1 j}\left(\tau_{1 j}\right) \ldots \ldots \ldots \ldots$

41 Experimental detector-detector correlations $C_{i j}\left(\tau_{i j}\right) \ldots \ldots \ldots$. . . . 86

$42 \quad$ Ratio of $C_{i j \mid 1}\left(\tau_{i j}\right)$ with $C_{i j}\left(\tau_{i j}\right) \ldots \ldots \ldots \ldots \ldots \ldots \ldots$

$43 \quad$ Ratio of $\int_{0}^{\infty} C_{1 i}\left(\tau_{1 i}\right) C_{1 j}\left(\tau_{i j}+\tau_{1 i}\right) d \tau_{1 i}$ with $\int_{0}^{\infty} C_{1 i j}\left(\tau_{1 i}, \tau_{i j}+\tau_{1 i}\right) d \tau_{1 i} \ldots \ldots \quad 89$

44 Ratio of $C_{n g \mid n}\left(\tau_{23}\right)$ and $C_{n g \mid g}\left(\tau_{23}\right)$ with $C_{n g}\left(\tau_{23}\right)$; and ratio of $C_{g n \mid n}\left(\tau_{23}\right)$ and

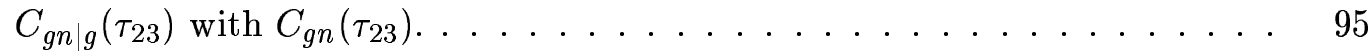

45 Ratio of $C_{n g \mid n}\left(\tau_{24}\right)$ and $C_{n g \mid g}\left(\tau_{24}\right)$ with $C_{n g}\left(\tau_{24}\right)$; and ratio of $C_{g n \mid n}\left(\tau_{24}\right)$ and

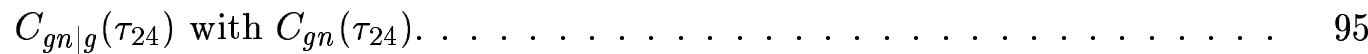

46 Ratio of $C_{n g \mid n}\left(\tau_{34}\right)$ and $C_{n g \mid g}\left(\tau_{34}\right)$ with $C_{n g}\left(\tau_{34}\right)$; and ratio of $C_{g n \mid n}\left(\tau_{34}\right)$ and $C_{g n \mid g}\left(\tau_{34}\right)$ with $C_{g n}\left(\tau_{34}\right) \ldots \ldots \ldots \ldots \ldots \ldots \ldots \ldots \ldots \ldots \ldots \ldots \ldots \ldots$

47 Ratio of $C_{n g}\left(\tau_{23}\right)^{2}$ with $C_{n g \mid n}\left(\tau_{23}\right)$ and $C_{n g \mid g}\left(\tau_{23}\right)$; and ratio of $C_{g n}\left(\tau_{23}\right)^{2}$ with $C_{g n \mid n}\left(\tau_{23}\right)$ and $C_{g n \mid g}\left(\tau_{23}\right) \ldots \ldots \ldots \ldots \ldots \ldots \ldots$

48 Ratio of $C_{n g}\left(\tau_{24}\right)^{2}$ with $C_{n g \mid n}\left(\tau_{24}\right)$ and $C_{n g \mid g}\left(\tau_{24}\right)$; and ratio of $C_{g n}\left(\tau_{24}\right)^{2}$ with $C_{g n \mid n}\left(\tau_{24}\right)$ and $C_{g n \mid g}\left(\tau_{24}\right)$. 
49 Ratio of $C_{n g}\left(\tau_{34}\right)^{2}$ with $C_{n g \mid n}\left(\tau_{34}\right)$ and $C_{n g \mid g}\left(\tau_{34}\right)$; and ratio of $C_{g n}\left(\tau_{34}\right)^{2}$ with $C_{g n \mid n}\left(\tau_{34}\right)$ and $C_{g n \mid g}\left(\tau_{34}\right) \ldots \ldots \ldots \ldots \ldots \ldots \ldots \ldots \ldots \ldots$

50 Ratio of $C_{n g \mid g}\left(\tau_{23}\right)$ with $C_{n g}\left(\tau_{23}\right)$; and ratio of $C_{g n \mid g}\left(\tau_{23}\right)$ with $C_{g n}\left(\tau_{23}\right) . \quad \ldots \quad 98$

51 Ratio of $C_{n g \mid n}\left(\tau_{23}\right)$ with $C_{n g}\left(\tau_{23}\right)$; and ratio of $C_{g n \mid n}\left(\tau_{23}\right)$ with $C_{g n}\left(\tau_{23}\right) . \quad . \quad 99$

52 Comparison of the source-detector correlations $C_{1 j}\left(\tau_{1 j}\right)$ between the 3-inch and 4 -inch detectors. . . . . . . . . . . . . . . . . 100

53 Comparison of the gamma peak of the source-detector correlations $C_{1 j}\left(\tau_{1 j}\right)$ between the 3 -inch and 4 -inch detectors. . . . . . . . . . . . . . . . 101

54 Comparison of the detector-detector correlations $C_{i j}\left(\tau_{i j}\right)$ between the 3-inch and 4 -inch detectors. . . . . . . . . . . . . . . . 102

55 Comparison of the gamma peak of the detector-detector correlations $C_{i j}\left(\tau_{i j}\right)$ between the 3 -inch and 4 -inch detectors. . . . . . . . . . . . . 102

56 Gamma-neutron-neutron triplets as a function of delay time $\tau_{i j} \ldots \ldots \ldots$. . 104 


\section{LIST OF SYMBOLS}

\section{Symbols from standard NDA in Chapter 2}

$\alpha$

$\lambda$

A

$D$

$f_{d}, f_{t}$

$f_{x}$

$m_{\text {Total }}$

$p(n)$

$q(n)$

$R+A$

$r(n)$

$S$

$T$

$W$

\section{General symbols}

ratio of $(\alpha, n)$ to spontaneous fission neutrons. (not to be confused with the Rossi-alpha as used below)

average singles detection rate

Accidental gate in a shift register

Doubles rate

doubles and triples gate fraction

mass fraction of isotope $x$ such as 235

total mass of plutonium

probability distribution of $n$ counts in the $R+A$ gate of the shift register

probability distribution of $n$ counts in the $A$ gate of the shift register

Reals plus accidental gate in a shift register

probability distribution of $n$ correlated counts in the $R+A$ gate of the shift register

Singles rate

Triples rate

shift register gate window in units of time

Rossi alpha. Related to $M$ and $k_{e f f}$ The symbol is also used to indicate the rate of $(\alpha, \mathrm{n})$ reactions in neutron multiplicity counting. 
$\chi(\tau)$

$\chi\left(E_{n}\right)$

$\Delta t$

$\delta(\tau)$

$\epsilon_{f}$

$\epsilon_{n}$

$\epsilon_{n}\left(E_{n}\right)$

$\epsilon_{g}$

$\epsilon_{n}(\tau) \chi(\tau)$

$\gamma$

$\gamma_{n i}$

$\gamma_{n s}$

$\gamma_{x}$

$\bar{\gamma}$ or $\gamma_{1}$

$\nu$

$\bar{\nu}$ or $\nu_{1}$

$\overline{\nu(\nu-1)}$ or $\nu_{2}$ fission spectrum of prompt neutrons as a function of detection time

fission spectrum of prompt neutrons as a function of energy time bin width

gamma-ray response of fast detector, approximated by a delta function

fission detection efficiency in the instrumented source

average neutron detection efficiency (neutrons detected per neutron emitted from a source)

neutron detection efficiency as a function of energy

average gamma detection efficiency (gamma rays detected per gamma rays emitted by a source)

time distribution of the detection of prompt neutrons from a spontaneous fission source

number of prompt gamma rays emitted from a fission

$\mathrm{n}$ th moment of the number of prompt gamma rays emitted from induced fission

$\mathrm{n}$ th moment of the number of prompt gamma rays emitted from spontaneous fission

number of gamma rays emitted from reaction $\mathrm{x}$. $\mathrm{i}$ - inelastic scatter, $\mathrm{r}$ - radioactive decay, $\alpha$ - alpha-n reaction

first moment or average number of prompt gamma rays emitted from a fission

number of prompt neutrons emitted from a fission

first moment or average number of prompt neutrons emitted from a fission

second moment of the number of prompt neutrons emitted from a fission 
$\overline{\nu(\nu-1)(\nu-2)}$ or $\nu_{3}$

$\overline{\nu_{i}}$ or $\nu_{i 1}$

$\nu_{n}, \nu_{s n}$ or $\nu_{i n}$

$\overline{\nu_{s}}$ or $\nu_{s 1}$

$\tau_{12}$

$\tau_{23}$

$\tau_{D}$

$\tau_{d}$

$\tau_{1 j}$

$c$

$C_{i j}\left(\tau_{i j}\right)$

$C_{i j k}\left(\tau_{i j}, \tau_{i k}\right)$

$C_{i j \mid k}\left(\tau_{i j}\right)$

$C_{i j}$

$C_{x y \mid z}\left(\tau_{i j}\right)$

$C_{x y}\left(\tau_{i j}\right)$ third moment of the number of prompt neutrons emitted from a fission

first moment of the number of prompt neutrons emitted from induced fission

$\mathrm{n}$ th moment of the number of prompt neutrons emitted from a fission

first moment of the number of prompt neutrons emitted from spontaneous fission

delay from fission to detection in detector 2

delay between detection in detector 2 and detector 3

detector die away time

detector dead time

delay from fission to detection in detector $j$

speed of light in a vacuum

time correlation between detectors $i$ and $j$

time bicorrelation between detectors $i, k$ and $k$

time correlation between detectors $i, j$ and $k$ with the time variable $\tau_{i k}$ integrated out

time correlation between detectors $i$ and $j$ with the time variable integrated out

time correlation between radiation $x$ in detector $i, y$ in detector $j$ and $z$ in detector $k$ with the time variable $\tau_{i k}$ integrated out

Correlation with radiation $x$ detected in detector $i$ and radiation $y$ detected in detector $j$, e.g., $C_{g n}\left(\tau_{i j}\right)$. Symbol $g$ represents a gamma, $n$ a neutron and $f$ represent fissions. 


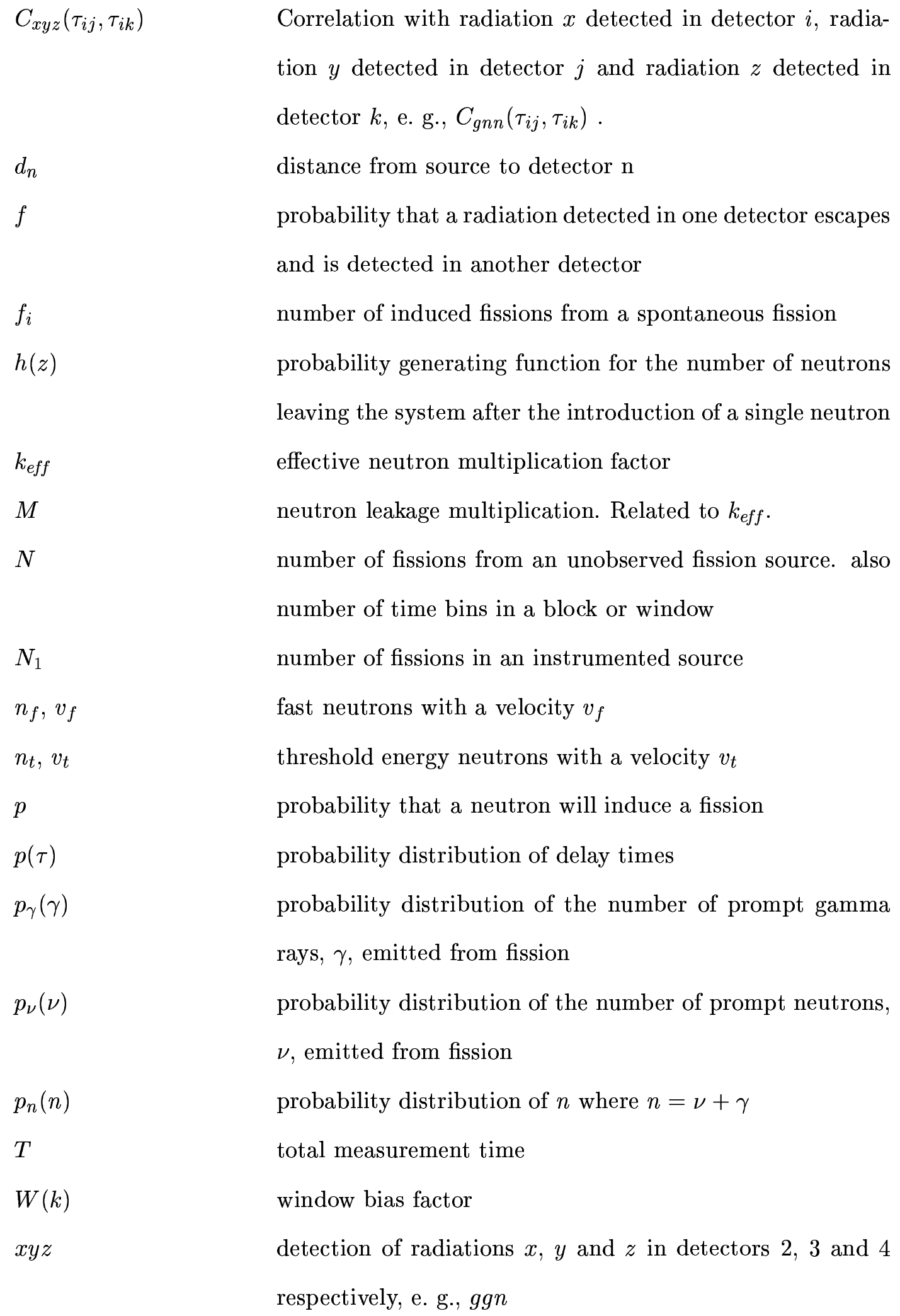




\section{SUMMARY}

The current practice of nondestructive assay (NDA) of fissile materials using neutrons is dominated by the ${ }^{3} \mathrm{He}$ detector. This has been the case since the mid 1980 s when Fission Multiplicity Detection (FMD) was replaced with thermal well counters and neutron multiplicity counting (NMC). The thermal well counters detect neutrons by neutron capture in the ${ }^{3} \mathrm{He}$ detector subsequent to moderation. The process of detection requires from 30 to $60 \mu \mathrm{s}$. As will be explained in Section 3.3 the rate of detecting correlated neutrons (signal) from the same fission are independent of this time but the rate of accidental correlations (noise) are proportional to this time. The well counters are at a distinct disadvantage when there is a large source of uncorrelated neutrons present from $(\alpha, \mathrm{n})$ reactions for example.

Plastic scintillating detectors, as were used in FMD, require only about 20 ns to detect neutrons from fission. One thousandth as many accidental coincidences are therefore accumulated. The major problem with the use of fast-plastic scintillation detectors, however, is that both neutrons and gamma rays are detected. The pulses from the two are indistinguishable in these detectors.

For this thesis, a new technique was developed to use higher-order time correlation statistics to distinguish combinations of neutron and gamma ray detections in fast-plastic scintillation detectors. A system of analysis to describe these correlations was developed based on simple physical principles. Other sources of correlations from non-fission events are identified and integrated into the analysis developed for fission events. A number of ratios and metrics are identified to determine physical properties of the source from the correlations. It is possible to determine both the quantity being measured and detection efficiency from these ratios from a single measurement without a separate calibration. To account for detector dead-time, an alternative analytical technique was also developed. 
As a demonstration of this technique, an experiment based on a ${ }^{252} C f$ source and three plastic scintillating detectors was conducted. The Nuclear Materials Identification System (NMIS), a system developed at ORNL, was used to analyze the time correlations for the experiment.

The mass of the ${ }^{252} \mathrm{Cf}$ source was estimated from three methods. The results of these methods are compared to an independent measurement of the ${ }^{252} C f$ mass at ORNL. The three methods include 1) the count rate of the ion-chamber containing the source; 2) ratios of the square of $g n$ pairs to either $g n \mid g$ or $g n \mid n$ combinations; and 3) finally the $C_{g n \mid n}$ correlations.

The number of fissions detected in the ion-chamber containing the ${ }^{252} C f$, adjusted for the fission detection-efficiency $\epsilon_{f}$, is the most reliable of these methods. The result from the ion-chamber method was about $0.13 \mu \mathrm{g} \pm 10 \%$. Of the two passive methods, the $C_{g n \mid n}$ correlation proved to be more accurate with an adjustment for neutron triplets. The ratio of the $g n$ correlation squared suffered from both $n n$ contamination in $C_{g n}$ as well as $n n n$ contamination in $C_{g n \mid g}$ and $C_{g n \mid n}$. The result from the $C_{g n \mid n}$ correlation was about $0.117 \mu \mathrm{g}$ $\pm 2 \%$ for the 3 -inch detector experiment and $0.115 \mu \mathrm{g} \pm 1 \%$ for the 4 -inch experiment. ${ }^{2}$ The independent measurement at ORNL resulted in values of $0.115 \mu \mathrm{g} \pm 3 \%$ and $0.114 \mu \mathrm{g}$ $\pm 3 \%$ at the time the 3 -inch and 4 -inch experiments were performed.

The main weakness of this technique as implemented in these experiments is low detection efficiency. In these experiments the detection efficiency was less than $2 \times 10^{-3}$. This detection efficiency can be improved with larger detectors. The advantage is the shorter correlation window. A window as short as $32 \mathrm{~ns}$ could be used. This is 1000 times shorter than that needed by well counters. The combination of low detection efficiency and a short correlation window would provide a great advantage in the case of a small amount of fissile material in the presence of a large $(\alpha, \mathrm{n})$ background.

\footnotetext{
${ }^{2} 3$-inch and 4-inch are the nominal sizes of the detectors used. The actual dimensions are given in the description of the experiment in Section 7.2.
} 



\title{
CHAPTER I
}

\section{INTRODUCTION}

"their material possessed the wonderful quality of being invisible to any man who was unfit for his office or unpardonably stupid."

\author{
Hans Christian Andersen \\ Fairy Tales and Stories (1837) \\ English Translation: H. P. Paull (1872)
}

Nondestructive assay (NDA) of nuclear materials is made possible by the fact that fissile and fertile transuranic (TRU) isotopes emit characteristic radiations. TRU isotopes typically decay by alpha decay. The even numbered isotopes also have significant spontaneous fission rates. NDA is generally divided into active and passive techniques. Passive techniques take advantage of the spontaneous radiations emitted by these isotopes. These techniques can be further subdivided by the type of radiation measured. These categories are gamma-ray assay, neutron assay, and calorimetric assay.

Fission Multiplicity Detection (FMD) is the name of the standard technique used in NDA of nuclear materials in the 1960s and 1970s. It was characterized by the use of fast plastic scintillating detectors. These systems were used in both active and passive mode. These FMD systems were eventually replaced by thermal well counters as the standard NDA technique. The thermal well counters use ${ }^{3} \mathrm{He}$ detectors embedded in a moderator. Among the passive neutron assay techniques, neutron multiplicity counting (NMC) in a thermal well counter is a preferred technique for the determination of fissile mass when spontaneous fission yields are significant. It is used in conjunction with gamma-ray spectroscopy to determine the isotopic composition of a sample. From the isotopic composition, NMC 
determines the fissile mass.

The major problem with the use of fast-plastic detectors as used in FMD is that both neutrons and gamma rays are detected. The pulses from the two are indistinguishable.[44] Recently, however, there is a renewed interest in using plastic scintillating detectors for FMD due to the higher count rates possible and the lower accidental coincidence rate. The availability of fast time analyzers make the distinguishability of neutron and gamma-ray events possible in fast-plastic detectors.

For this thesis, a new technique was developed to use higher-order time correlation statistics to distinguish combinations of neutron and gamma ray detections in fast plastic detectors. A system of analysis to describe these correlations from fission events was developed based on simple physical principles. Other sources of correlations from nonfission events were identified and integrated into the analysis developed for fission events. A number of ratios and metrics were identified to determine physical properties of the fissile material from the correlations. The analysis does not explicitly includes combinations of events such as fissions followed by inelastic scattering of the neutrons in the matrix materials. The thesis, instead provides qualitative insight into the interpretation of empirical data which include these effects.

Fissions followed by further fissions in the multiplication process, on the other hand, is explicitly included in the analysis. This analysis will be an extension of the early treatment of the multiplication process proposed by Böhnel which is still used in NMC today.[6]

As a demonstration of this technique, an experiment based on a ${ }^{252} C f$ source and three plastic scintillating detectors was conducted. The Nuclear Materials Identification System (NMIS)[35], a system developed at ORNL, was used to analyze the time correlations for the experiment. The new technique was first used with an instrumented ${ }^{252} \mathrm{Cf}$ source, and then extended to a non-instrumented source to demonstrate the relation between the two configurations. Various data about the source were extracted and analyzed from the time correlation statistics. 
The current and previous techniques of passive neutron NDA are first reviewed in Chapter 2. How the time correlations are calculated are described by example in Chapter 3 . The mathematics necessary to represent the time correlations is then reviewed in that chapter. An analytical representation for the correlations between the source and a detector, between two detectors, between the source and two detectors, and finally between three detectors resulting from a fission event is developed in Chapter 4. From this analysis, the temporal separation of the gamma and neutron combinations becomes apparent. Various ratios of these correlations are presented. In addition various non-fission sources of correlated detections are described and related to the analysis of the correlations from fission. To account for detector dead-time, an alternative analytical technique will be presented in Chapter 6 . The experimental setup for the ${ }^{252} C f$ measurement will be presented in Chapter 7 . The results of the experiment will then be presented in Chapter 8, followed by conclusions in Chapter 9. A derivation of the factorial moments for neutron and gamma-ray coincident detections from multiplying material using probability generating functions is given in Appendix A. 


\section{CHAPTER II}

\section{CURRENT AND PAST PRACTICES OF PASSIVE NEUTRON AND FISSION MULTIPLICITY COUNTING TECHNIQUES FOR NDA}

This chapter will describe the current techniques of passive neutron counting for NDA with thermal well counters including the more recent innovation of Time Interval Analysis (TIA). Fission multiplicity detection (FMD) will then be briefly described along with the previous NMIS implementation of multiplicity.

In $\mathrm{NDA}^{1}$ it is the quantity of an unknown fissile mass that is of interest. In addition to this unknown, the chemical composition, mixture and environment of the mass may also be unknown. Because of these other unknowns, neutrons may be generated from $(\alpha, n)$ reactions rather than fission, and the neutron detection efficiency may not be well known. The techniques used to overcome these difficulties will be described.

\section{$\underline{2.1 \quad \text { Detectors }}$}

The nature of the detectors used in the current practice of passive neutron counting for NDA must be contrasted with the fast, plastic-scintillating detectors used in this research. The detectors used in current techniques are neutron well counters. These well counters are comprised of ${ }^{3} \mathrm{He}$ tubes embedded in an annular polyethylene moderator. The sample being measured is placed in the well of the annulus which is lined with cadmium to decouple the sample from the moderator. ${ }^{3} \mathrm{He}$ detectors operate through the ${ }^{3} \mathrm{He}(n, p){ }^{3} \mathrm{H}$ thermal capture reaction. As a consequence these detectors do not detect gamma rays. Furthermore, once a neutron is detected it is removed from the system.

\footnotetext{
${ }^{1}$ See References [45] and [46] for a good overview of NDA.
} 
The fast, plastic-scintillating detectors operate on proton recoil for neutron detection. Gamma rays are detected as well as neutrons, and a detection of a neutron or gamma ray does not remove it from the system. The neutron detection efficiency for the well counters can be as high as $50 \%$ or more. By contrast the efficiency of the 4-inch detectors used in this research is less than $1 \%$ when the solid angle is considered. The detection efficiency of the well counter is greatest at about $1 \mathrm{MeV}$. Relatively high detection efficiency extends down to several $\mathrm{keV}$ or lower. The fast plastic scintillators are blind below the threshold setting which was set at $1 \mathrm{MeV}$ in this research.

The time response between the two types of detectors is also entirely different. The time response of the well counters is characterized by a single exponential die-away with a time constant on the order of $50 \mu \mathrm{s}$. The detector die-away time is a characteristic of the thermal detector moderator. The time response of the fast plastic detectors is dominated by the time of flight of the radiation. For the threshold neutrons with a velocity of $1.4 \mathrm{~cm} / \mathrm{ns}$ this time is $17 \mathrm{~ns}$ at a distance of $23 \mathrm{~cm}$.

\subsection{Thermal well and shift register techniques}

Total neutron counting, coincidence counting and neutron multiplicity counting (NMC) are all used in NDA. Although NMC in a sense encompasses the other two methods, the other methods are still used and can produce better results. The choice of method depends on the nature of four unknowns: total mass, $(\alpha, n)$ rate, multiplication, and detection efficiency. It is the total mass of plutonium $m_{\text {Total }}$ that is determined from a passive neutron measurement. The isotopic composition of the plutonium is determined by another method. This other method would typically be gamma spectroscopy. The composition is expressed as mass ratios $\left(f_{238}, f_{240}, f_{242}\right)$. One might observe that the total mass in milligrams could be determined from the total neutron count rate per second $S$ using the data in Table $1 .^{2} \quad$ The symbol $S$ is used because this count rate is identical to the singles rate in $\mathrm{NMC}$.

\footnotetext{
${ }^{2}$ This data is from Reference [45].
} 


$$
m_{\text {Total }}(m g)=\frac{S}{\epsilon_{n}\left[2.59\left(\frac{n}{m g \cdot s}\right) f_{238}+1.02\left(\frac{n}{m g \cdot s}\right) f_{240}+1.72\left(\frac{n}{m g \cdot s}\right) f_{242}\right]}
$$

Equation (1) assumes that there are no $(\alpha, n)$ reactions, and no multiplication. Suppose however that some of the plutonium is in oxide form. The neutron yield from $(\alpha, n)$ reactions can be considerable. Neutron coincidence counting was developed to measure $m_{\text {Total }}$ when the $(\alpha, n)$ reaction rate was unknown. Neutrons from the $(\alpha, n)$ reaction are always generated in singles. Neutrons from spontaneous fission, on the other hand, are generated in multiplets $\nu$ with a probability density of $p(\nu)$. The effect of multiplication is generally estimated through calibration standards.

Coincidence counting is essentially identical to determining the doubles rate $D$ in multiplicity counting. The explanation of coincidence counting will therefore be treated along with multiplicity counting. ${ }^{3} \quad$ Although multiplicity counting can determine the multiplication directly, coincidence counting with a calibration standard can produce better results.

Table 1: Spontaneous fission data

\begin{tabular}{ccc}
\hline Isotope & $\begin{array}{c}\text { Spontaneous } \\
\text { fission yield } \\
\left(\frac{n}{m g \cdot s}\right)\end{array}$ & $\bar{\nu}$ \\
\hline & & \\
${ }^{238} \mathrm{Pu}$ & 2.59 & 2.21 \\
${ }^{240} \mathrm{Pu}$ & 1.02 & 2.16 \\
${ }^{242} \mathrm{Pu}$ & 1.72 & 2.15 \\
${ }^{252} \mathrm{Cf}$ & $2.34 \times 10^{9}$ & 3.757 \\
\hline
\end{tabular}

The calculation of $m_{\text {Total }}$ in Equation 1 is based on spontaneous fission yields. It was assumed that there was no multiplication in the plutonium, i. e., that the spontaneous fissions did not induce more fissions. It also assumes that the detection efficiency $\epsilon_{n}$ is known. This efficiency can be affected by the composition of the sample which might not

\footnotetext{
${ }^{3} \mathrm{~A}$ more comprehensive introduction to multiplicity counting can be found in reference [16]. In addition, reference [1] provides insight and historical perspective.
} 
be well known. One of these additional unknowns can be determined if, in addition to the doubles rate $D$ in coincident counting, the triples rate $T$ is also determined. This extension is in essence multiplicity counting. Both the doubles $D$ and triples rate $T$ are determined from shift register statistics.

A typical shift register as shown in Figure 1 might have 128 bits and operate on a 4 $\mathrm{MHz}$ clock. Each bit would therefore represent $0.25 \mu$ s. The gate window $W$ would be 32 $\mu$ s which is the total time required for a bit to travel through the shift register. A pulse from the detector sets the first bit in the shift register. This bit is shifted one bit at a time down the register every $0.25 \mu \mathrm{s}$. A counter increments by one for each bit that enters the shift register and decrements by one for each bit that exits the shift register $32 \mu$ s later. This counter therefore contains the number of pulses in the shift register at any time which represents the number of detected pulses which occurred in the previous $32 \mu \mathrm{s}$.

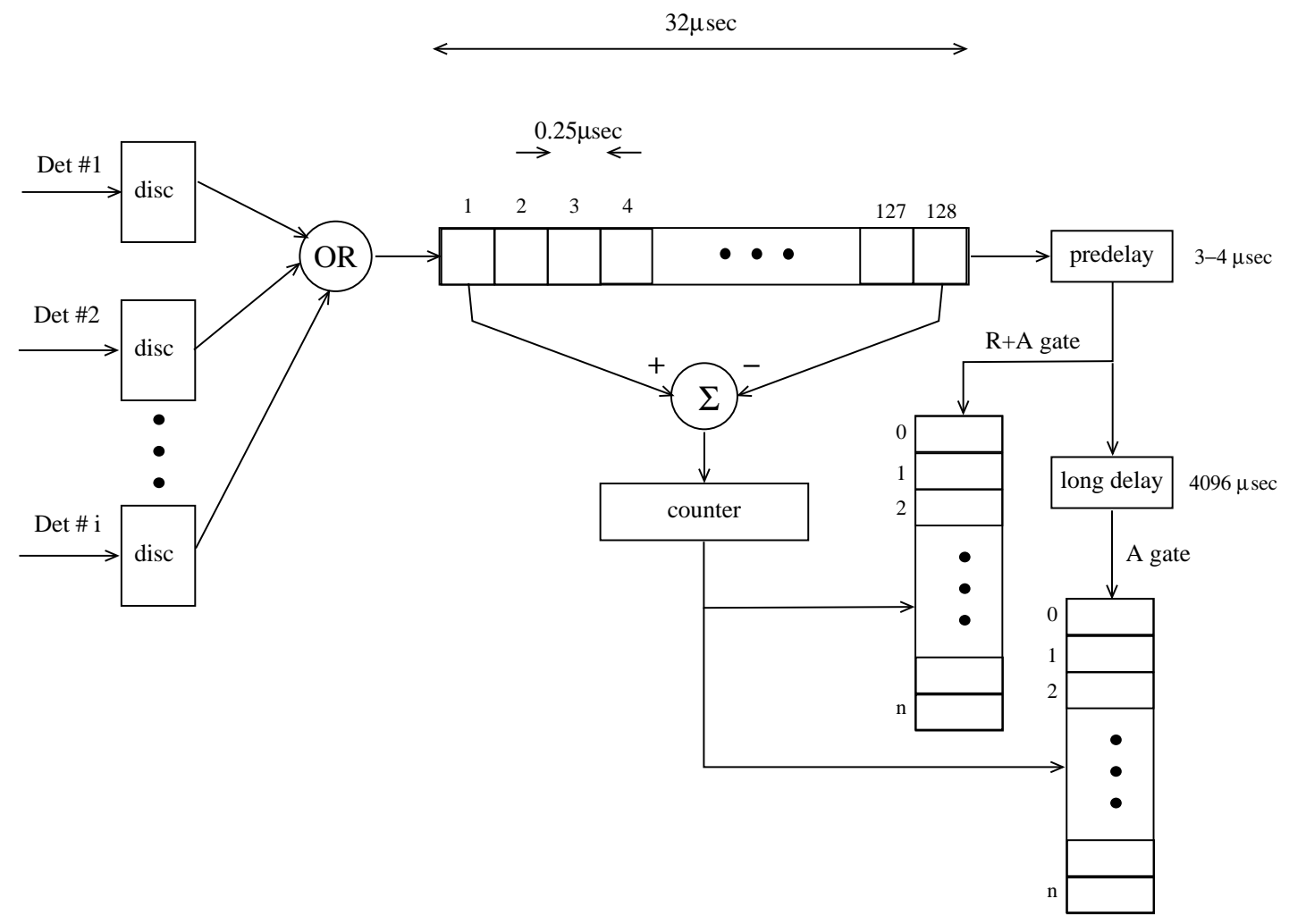

Figure 1: Shift register functional diagram. 
The content of the counter must be tallied at various times. Tallying the shift register is often referred to as triggering the gate. There are essentially three methods for choosing this time. These approaches are referred to as 1) signal-triggered, 2) randomly-triggered, and 3) combined-triggered. In the signal-triggered method, the content of the shift register is tallied when each pulse exits the shift register. The result is the number of pulses following every pulse within the gate window $W$. Typically the tally is triggered after a short delay of 3 to $4.5 \mu$ s called a pre-delay. This pre-delay results in shifting the closest pulses off the shift register before the tally is made. These pulses tend to be biased by detector dead time. The purpose of the pre-delay is to remove this bias. A gate triggered in this manner is called the $R+A$ or reals plus accidentals gate. For pulses occurring at an average rate $\lambda$, the shift register will contain $\lambda W$ pulses on average. If $\lambda W$ is small and the pulses are random, the number of pulses $n$ in the shift register will follow Poisson statistics. When pulses arise from fission neutrons however, they tend to be clustered in time because of the multiplicity of neutron emission. This clustering gives rise to the real coincidences in the $R+A$ gate. The average number of pulses in the shift register will then be greater than $\lambda W$.

Alternatively, the gate could be triggered either periodically or randomly (independent of the pulse times). This approach is the randomly-triggered method. In either case, the gate is triggered randomly in time from the detector pulses. The average number of pulses will be $\lambda W$ which is the accidental rate. The actual distribution of $n$ pulses in the register will not follow Poisson statistics if the pulses tend to be clustered in time because of fission multiplicity. The real coincidences can also be determined from this approach from the deviation from Poisson statistics. This method comes from the Feynman variance method.

As the name implies, the combined approach has both signal and random triggering. Typically however, the random triggering is accomplished by triggering the $A$ gate after a long but constant delay after the $R+A$ gate. The delay for the $A$ gate is set sufficiently long to allow all correlated events to die away. A typical value for this delay might be $4096 \mu \mathrm{s}$. Although in reality the $A$ gate is signal triggered, it is assumed that all the real correlated 
events have died away.

Once the measurement is accomplished in the combined approach, there will be two distributions of shift register counts. One distribution is for the $R+A$ gate, the other is for the $A$ gate. The distributions contain tallies of the number of times that $0,1,2, \ldots n$ bits were set in the shift register at the time it was triggered. Properly normalized by the total counts, we can call these distributions $p(n)$ from the $R+A$ gate and $q(n)$ for the $A$ gate. These distributions represent the probability of finding $0,1,2, \ldots$ counts in the shift register at the appropriate trigger times. From these distributions, the singles, doubles, and triplets are computed.

The distribution that we want is the probability that $n$ correlated neutrons are in the shift register. This probability is usually called $r(n)$. The probability of finding no counts in the $R+A$ gate is $p(0)=r(0) q(0)$. That is, the probability of finding no correlated counts and no accidental counts. One count in the $R+A$ shift register can come from one correlated count and no accidentals or from one accidental and no correlated counts. The probability of this occurring is therefore $p(1)=r(0) q(1)+r(1) q(0)$. The probability of two counts is $p(2)=r(0) q(2)+r(1) q(1)+r(2) q(0)$ and so on. These probabilities are related in general by

$$
p(n)=\sum_{i=0}^{n} r(i) q(n-i) .
$$

The probability distribution $r(n)$ can be solved recursively. From the preceding paragraph, we know that $r(0)=\frac{p(0)}{q(0)}$. The probability of one real can then be solved from $r(0)$ as $r(1)=\frac{p(1)-r(0) q(1)}{q(0)}$. Continuing the process results in the recursive formula for $r(n)$ :

$$
r(n)=\frac{1}{q(0)}\left[p(n)-\sum_{i=0}^{n-1} r(i) q(n-i)\right] .
$$

Of course when $n$ is zero, no correlated counts are in the shift register when it is triggered. A doublet results when one pulse in the shift register is correlated with the trigger. Therefore $n=1$ is indicative of a doublet or correlated pair.

The singles, doubles and triples $(S, D, T)$ are then computed from the moments of the 
$r(n)$ distribution.[25] These moments are defined as

$$
r_{n}=\sum_{i=n}^{\infty}\left(\begin{array}{c}
i \\
n
\end{array}\right) r(i)=\sum_{i=n}^{\infty} \frac{i !}{n !(i-n) !} r(i) .
$$

The first three of these moments are

$$
\begin{aligned}
& r_{0}=\sum_{i=0}^{\infty} r(i)=1 \\
& r_{1}=\sum_{i=1}^{\infty} i r(i)=\bar{i} \\
& r_{2}=\sum_{i=2}^{\infty} i(i-1) r(i)=\frac{\overline{i(i-1)}}{2} .
\end{aligned}
$$

If we let $R$ be the total number of gate triggers in the shift register or this total divided by measurement time for a rate, the singles, doubles and triples are

$$
\begin{aligned}
& S=R r_{0}=R \\
& D=S r_{1} \\
& T=S r_{2} .
\end{aligned}
$$

These values can be and typically are computed directly from the moments of the $p(n)$ and $q(n)$ distributions. In addition it is also possible to compute $S, D$ and $T$ from the background $q(n)$ alone using the Feynman variance technique.

The singles, doublets and triplets can then be related to the actual physical parameters of the sample by the neutron multiplicity point equations.[21]

$$
\begin{aligned}
& S=N \epsilon_{n} M \nu_{s 1}(1+\alpha) \\
& D=\frac{N \epsilon_{n}^{2} f_{d} M^{2}}{2}\left[\nu_{s 2}+\left(\frac{M-1}{\nu_{i 1}-1}\right) \nu_{s 1}(1+\alpha) \nu_{i 2}\right] \\
& T=\frac{N \epsilon_{n}^{3} f_{t} M^{3}}{6}\left[\nu_{s 3}+\left(\frac{M-1}{\nu_{i 1}-1}\right)\left[3 \nu_{s 2} \nu_{i 2}+\nu_{s 1}(1+\alpha) \nu_{i 3}\right]\right. \\
& \left.+3\left(\frac{M-1}{\nu_{i 1}-1}\right)^{2} \nu_{s 1}(1+\alpha) \nu_{i 2}^{2}\right]
\end{aligned}
$$

where

$N$ is the spontaneous fission rate of ${ }^{240} \mathrm{Pu}$, 
$\epsilon_{n}$ is the neutron detection efficiency,

$M$ is the neutron leakage multiplication defined as $M=\frac{1-p}{1-k_{e f f}}$, where $k_{e f f}=p \overline{\nu_{i}}$ is the neutron multiplication factor,

$\alpha$ is the $(\alpha, n)$ to spontaneous fission neutron ratio, ${ }^{4}$

$f_{d}, f_{t}$ are the doubles and triples gate fraction respectively, (See Section 2.3 for further discussion on these factors) and

$\nu_{s n}, \nu_{i n}$ are the $\mathrm{n}$ th reduced factorial moments of the spontaneous and induced fission distributions respectively. These reduced moments are defined similar to Equation 4 but without the $n$ ! in the denominator, which then appears explicitly in the equations.

Usually three of the four variables, $N, \epsilon_{n}, M$, or $\alpha$ are solved from $S, D$, and $T$. The effective mass of ${ }^{240} \mathrm{Pu}$ is then calculated from the fission rate $N$ and the specific fission rate of ${ }^{240} \mathrm{Pu}$. Finally the total mass of $\mathrm{Pu}$ is

$$
m_{\text {Total }}=\frac{m_{240}}{\left(2.54 f_{238}+f_{240}+1.69 f_{242}\right)} .
$$

The final value of $m_{\text {Total }}$ may still be biased if the sample being measured does not meet the assumptions of the point equations. Calibration procedures may be needed to remove this bias. In addition, corrections may also be needed to account for detector dead-time.

\section{$\underline{2.3}$ Time Interval Analysis (TIA)}

A new approach to neutron multiplicity analysis has been recently proposed by Baeten.[1, $7,2,3,4,5]$ It departs from the traditional shift register approach in favor of time interval analysis (TIA). In this sense it is similar to the time correlations produced by NMIS. In terms of NMIS signatures, it is based on the detector autocorrelation for doublets and a detector auto bicorrelation for triplets. It differs from NMIS in that the detector is a thermal well used in the traditional multiplicity techniques as described above.

\footnotetext{
${ }^{4}$ The symbol $\alpha$ is also used in Chapter 5 for the Rossi-alpha. Both uses of $\alpha$ are so well established that the conflict will be tolerated.
} 
Although TIA is a new approach to multiplicity analysis, virtually identical equations were derived by Hansen in 1968.[22] Baeten uses the same definition of the delay variables, i. e., $\tau_{1}=t_{2}-t_{1}$ and $\tau_{2}=t_{3}-t_{2}$. An arguably more natural definition for $\tau_{2}$ would refer it back to the first detection also, which would make $\tau_{2}=t_{3}-t_{1}$. One difference, however, is that Hansen casts his equations in terms of the Rossi-alpha and includes multiplication terms. Baeten's equations are in terms of the detector die-away $\tau_{D}$ and does not include multiplication. Baeten's treatment of multiplication will be revisited briefly in Section 5.1.

The probability density of detecting a neutron introduced into the system at time zero by such a thermal detector as a function of time, given that it is detected, is $p(t)=\frac{e^{-\frac{t}{\tau_{D}}}}{\tau_{D}}$. The variable $\tau_{D}$ is the detector die-away time. The unobserved source-detector correlation would then be

$$
C_{12}(t)=\lambda+\frac{N \epsilon_{n} \bar{\nu}}{\tau_{D}} e^{-\frac{t}{\tau_{D}}}
$$

where $\lambda$ is the average background counts rate. If we assume that the only source of this background is uncorrelated accidentals arising from spontaneous fission and neutrons detected from $(\alpha, n)$ reactions, $\lambda=N \epsilon_{n} \bar{\nu}+N_{\alpha}$. The correlation $C_{12}(t)$ can be integrated over the measurement time to determine the number of singles $S$

$$
\begin{aligned}
S & =\frac{\int_{0}^{t_{m}} C_{12}(t) d t}{t_{m}}=N \epsilon_{n} \bar{\nu}+N_{\alpha}+\frac{N \epsilon_{n} \bar{\nu}}{t_{m}} \\
& \approx N \epsilon_{n} \bar{\nu}(1+\alpha)
\end{aligned}
$$

where $\frac{N \epsilon_{n} \bar{\nu}}{t_{m}}$ is virtually zero and $\alpha=\frac{N_{\alpha}}{N}$.

In such a system, the detector autocorrelation will be

$$
C_{22}\left(\tau_{1}=t_{2}-t_{1}\right)=\lambda^{2}+\frac{N \epsilon_{n}^{2} \overline{\nu(\nu-1)}}{2 \tau_{D}} e^{-\frac{\tau_{1}}{\tau_{D}}}
$$

where $t_{2}$ and $t_{1}$ are the times of two distinct correlated detections. In TIA this autocorrelation is called the one dimensional Rossi-alpha. This is somewhat of a misnomer because the die-away time $\tau_{D}$ is purely a characteristic of the detector. It has little to do with the reactivity of the sample being measured.

This autocorrelation can be related to the shift register statistics by integrating the time variable $\tau_{1}$ over the shift register gate window $W$. The doubles rate is $\frac{N \epsilon_{n}^{2} \overline{\nu(\nu-1)}}{2}$ provided 
$W>>\tau_{D}$. If this condition is not valid, a factor of $\left(1-e^{\frac{W}{\tau_{D}}}\right)$ remains. This factor is $f_{d}$ in Equation 6.

The auto-bicorrelation can also be computed for the thermal-well detector. It is a function of two delay variables $\tau_{1}=t_{2}-t_{1}$ and $\tau_{2}=t_{3}-t_{2}$. It will be comprised of accidental triplets, correlated pairs and one accidental, and correlated triplets.

First, the correlated triplet is

$$
\int_{0}^{\infty} \frac{N \epsilon_{n}^{3} \overline{\nu(\nu-1)(\nu-2)}}{\tau_{D}^{3}} e^{-\frac{3 t_{1}+\tau_{1}+\tau_{2}}{\tau_{D}}} d t_{1}=\frac{N \epsilon_{n}^{3} \overline{\nu(\nu-1)(\nu-2)}}{3 \tau_{D}^{2}} e^{-\frac{\tau_{1}+\tau_{2}}{\tau_{D}}} .
$$

There are three accidentals, at times $t_{3}, t_{2}$ or $t_{1}$, which can form triplets with correlated pairs. These correlated pair, accidental triplets are

$$
\lambda \frac{N \epsilon_{n}^{2} \overline{\nu(\nu-1)}}{2 \tau_{D}}\left(e^{-\frac{\tau_{1}}{\tau_{D}}}+e^{-\frac{\tau_{1}+\tau_{2}}{\tau_{D}}}+e^{-\frac{\tau_{2}}{\tau_{D}}}\right)
$$

respectively. The accidental triplet rate is then $\lambda^{3}$.

Combining these three components yields

$$
\begin{aligned}
C_{222}\left(\tau_{1}, \tau_{2}\right) & =\lambda^{3}+\frac{N \epsilon_{n}^{2}}{\tau_{D}}\left[\frac{\lambda \overline{\nu(\nu-1)}}{2}\left(e^{-\frac{\tau_{1}}{\tau_{D}}}+e^{-\frac{\tau_{1}+\tau_{2}}{\tau_{D}}}+e^{-\frac{\tau_{2}}{\tau_{D}}}\right)\right. \\
& \left.+\frac{\epsilon_{n} \overline{\nu(\nu-1)(\nu-2)}}{3 \tau_{D}} e^{-\frac{\left(2 t_{1}+t_{2}\right)}{\tau_{D}}}\right] .
\end{aligned}
$$

The triplets rate from the shift register approach is related to the correlated triplets and can be found by integrating over both delay variables, $\tau_{1}$ and $\tau_{2}$, from 0 to $W$. With the provision that $W>>\tau_{1}, \tau_{2}$, the triplet rate becomes

$$
T=\frac{N \epsilon_{n}^{3} \overline{\nu(\nu-1)(\nu-2)}}{6} .
$$

The other quantities from shift register analysis having already been found are

$$
\begin{aligned}
& S=N \epsilon_{n} \bar{\nu}(1+\alpha) \\
& D=\frac{N \epsilon_{n}^{2} \overline{\nu(\nu-1)}}{2} .
\end{aligned}
$$

Compare these equations for $S, D$ and $T$ to those of Equation 6 . These equations were derived under the assumption that there was no multiplication. Therefore $M=1$. It was 
assumed that $W>>\tau_{D}$ so that $f_{d}=f_{t}=1$ also. Substituting these values into Equation 6 reconciles the equations. The treatment of multiplication in TIA will be continued in the discussion in Chapter 5.

\section{$2.4 \quad$ Fission Multiplicity Detectors (FMD)}

Previous to the current practice of counting in thermal well counters, fast plastic-scintillation detectors were used in a technique called fission multiplicity detection (FMD). ${ }^{5}$ These systems were popular in the 1960s and 1970s. Several of these systems were commercially available. One of the earliest of these was the Isotopic Source Assay System (ISAS) developed by Gulf General Atomics. It was used in passive mode for the assay of plutonium or with a neutron source for the assay of uranium. Another commercial system was the Isotopic Source Adjustable Fissionometer (ISAF) designed by IRT Corporation formerly Gulf Radiation Technology. It used an Am-Li source in active mode. There were two versions of the Random Driver. One was from Los Alamos Scientific Laboratory. It used an Am-Li source. The other was from National Nuclear Corporation and used a Pu-Li source in active mode.

These systems all used two or four fast plastic detectors sensitive to both fast neutrons and gamma rays. Coincidences were counted between detectors. The analysis used was very much like that found in Chapters 4 and 6 of this thesis. Gamma rays were not explicitly treated however. The analysis was restricted to neutrons. In the case of LANL's Random Driver, the detectors were shielded with $5 \mathrm{~cm}$ of lead to suppress gamma detections. As will be shown in Section 4.6.2, the lead shields will produce correlations through inelastic scattering. In addition the gamma-gamma peak in the detector-detector cross correlation was blocked.

Multiplication was not considered in the analysis for FMD. Apparently there was heavy reliance on calibration standards to take multiplication into account. The large plastic scintillators used in FMD must have increased multiplication through reflection back into

\footnotetext{
${ }^{5}$ For a description of FMD see [46] pages 174 to 178 and [20] at paragraphs 8.6, 8.7 and 9.5.
} 
the sample. Cadmium is used in the thermal well counter to decouple the detector from the sample.

In a comparison with the active well the major disadvantage of the Random Driver was its bulk. It weighed a metric ton, which was ten times heavier than active well.

\section{$\underline{2.5}$ NMIS multiplicity}

A kind of multiplicity capability has been previously incorporated into NMIS.[37, 41, 51] This section will describe this implementation. In passive mode, a selectable number of the five detector channels can be treated as if it were one channel. An $R+A$ register is simulated with each detection being a trigger. The window length and pre-delay are definable in the software. In the multiplicity mode the block ${ }^{6}$ is disregarded as a barrier. The window can cross between blocks. For the $A$ register the beginning of each block is treated as the trigger. This approach is equivalent to periodic triggering. From the $R+A$ and $A$ gates the $p(n)$ and $q(n)$ distributions are generated. The distribution of reals $r(n)$ is generated from these recursively and the factorial moments of $r(n)$ computed directly from the distribution. NMIS essentially mimics a traditional shift register albeit capable of $1 \mathrm{~ns}$ time bins. If it were hooked up to a well counter, one would have an ordinary multiplicity system.

An active mode has also been implemented. In this mode only channel 1 acts as the trigger rather than all channels. In this mode the ${ }^{252} \mathrm{Cf}$ ion-chamber is connected to this channel. This differs from current implementations of active multiplicity in which the active source is not observed and therefore cannot act as a trigger.

The fast plastic scintillating detectors used in NMIS make these multiplicities entirely different from traditional multiplicity counting. Unlike the ${ }^{3} \mathrm{He}$ detectors used in traditional multiplicity, the plastic scintillators also detect gamma rays. So the multiplicity is a combined neutron and gamma multiplicity. In addition, the fast detectors are blind to neutrons below the threshold energy for neutrons, usually set at $1 \mathrm{MeV}$. Neutrons are not removed from the system on detection. This can cause a single radiation to be counted more

\footnotetext{
${ }^{6} \mathrm{~A}$ block is the term used for a periodic window.
} 
than once or to be reflected back into the sample. A more complete comparison between the detectors is described previously in Section 2.2 .

Why not simply use a combined gamma-neutron multiplicity as the assay measurement? First, there is nothing analogous to the point equations (Equation 6) used in the traditional multiplicity approach to relate the combined multiplicity to effective mass, $(\alpha$, n) rate, multiplication and detector efficiency. Second, although the fast plastic scintillators detect copious gamma rays, they are not especially well suited for that purpose. Because of the low $\mathrm{Z}$ of the detectors, the probability of interaction is low and furthermore the interactions that do occur are primarily Compton scattering interactions. So for a Compton scattering event, there is a substantial probability that the energy deposited will be below the threshold for gamma rays. Third, the gamma multiplicities are not well known. Finally, gamma multiplicities have numerous ill-behaved characteristics which make consistent measurements difficult. Gamma multiplicities are more energy dependent than neutrons.[8] Therefore small changes in the discriminator threshold will change the apparent multiplicity. Attenuation of the gamma rays, which is also energy dependent, will change the observed multiplicities. Many other events besides fission produce multiplicities of gamma rays. The beta decay of the fission products typically emit a cascade of several gamma rays. Not all of these problems are eliminated by temporal neutron-gamma separation, but they can at least be isolated and treated separately. 


\section{CHAPTER III}

\section{TIME ANALYSIS AND DEFINITION OF TIME CORRELATION}

The purpose of this chapter is to bridge the gap between the experiment and the mathematical analysis. First the algorithm used by the NMIS time-analyzer will be described with simple examples. The mathematics needed to represent the correlation data will then be described.

The input to the NMIS time-analyzer electronics comes from the output of a discriminator, a constant fraction discriminator (CFD). For now, assume that the discriminator signals the time of a detection event with a 0 to 1 logic transition. At this point, the signal is a continuous variable of time. The NMIS time-analyzer then effectively converts this continuous time signal into a discrete time variable by assigning it to a time bin of width $\Delta t$ in a block of data with $N$ time bins. Both $\Delta t$ and the block size $N$ are adjustable. Typically $\Delta \mathrm{t}=1 \mathrm{~ns}$ and $N=512$. The length of a block is then $512 \mathrm{~ns}$ or a little over a half a microsecond. Compare this time to the $\Delta \mathrm{t}$ of $0.25 \mu$ s for the typical shift-register. There are five input channels numbered 1 through 5 . The fundamental datum of NMIS is the time bin or bins in a block which contains a detection, if any, in each of five parallel channels. The blocks are parallel in the sense that they all begin and end at the same time. To put these parameters in perspective, a typical ${ }^{252} \mathrm{Cf}$ ionization chamber source will count $3 \times 10^{5}$ fissions per second. That means on average, one in 6.5 blocks will see a source fission. The detector count rates are much lower. At one meter from such a source, a 4-inch detector might register 1,000 counts per second. The detection rate is then less than one in a thousand blocks. The probability that a block from this detector will contain two uncorrelated detections (assuming no dead-time) is about $10^{-7}$.

The NMIS time-analyzer then computes and tallies the time between detections in 
all pairs and triplets of detectors. These are the correlations. They include blocks with themselves for the autocorrelations. All of the pairs and triplets are shown in Table 2.

Table 2: Correlation data accumulated by NMIS

\begin{tabular}{|c|c|c|}
\cline { 2 - 3 } \multicolumn{1}{c|}{} & correlation & bicorrelation \\
\hline \multirow{5}{*}{ Auto- } & 11 & 111 \\
& 22 & 222 \\
& 33 & 333 \\
& 44 & 444 \\
& 55 & 555 \\
\hline Cross- & 12 & 123 \\
& 13 & 124 \\
& 14 & 125 \\
& 15 & 134 \\
& 23 & 135 \\
& 24 & 145 \\
& 25 & 234 \\
& 34 & 235 \\
& 35 & 245 \\
& 45 & 345 \\
\hline
\end{tabular}

\subsection{NMIS Correlations - simple examples}

A few simple examples of how correlations are computed will now be described with a five bin block. Figure 2 shows channels 1,2 and 3 . Also shown is $C_{11}, C_{12}, C_{13}$ and $C_{123}$. For the autocorrelations, every detection is tallied in bin zero. All pairs of detections in a single block of the channel are tallied in the bin corresponding to the time difference between the two detections. The correlation $C_{13}$ is shown vertically beside $C_{123}$ to show how $C_{123}$ is related to $C_{12}$ and $C_{13}$. Notice that in the first case (a), $C_{13}$ has no detections. Therefore there are no triplets and $C_{123}$ will contain all zeros. The second case (b) shows two detections in channel 1 and one detection each in channels 2 and 3 . Notice that in $C_{123}$ two triplets are tallied. All of the doubles and triples are tallied block by block and accumulated.

Could the triples be computed as an outer product of the doubles correlations after all 


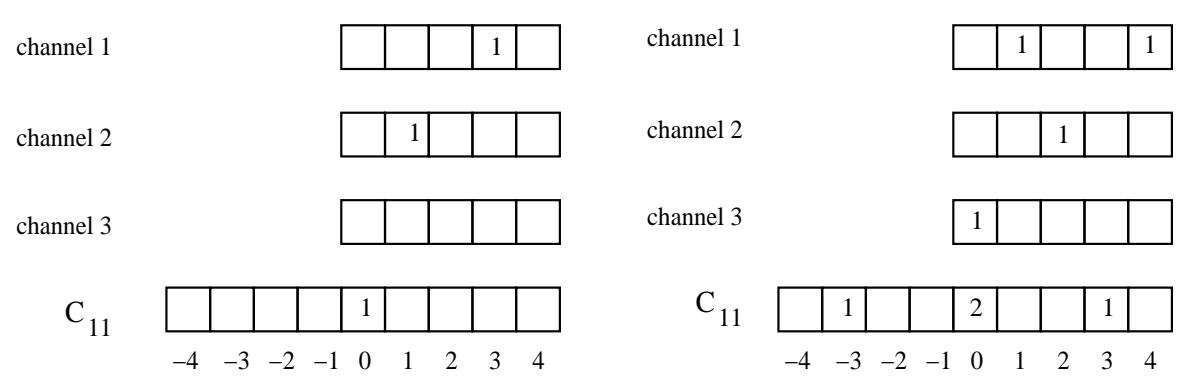

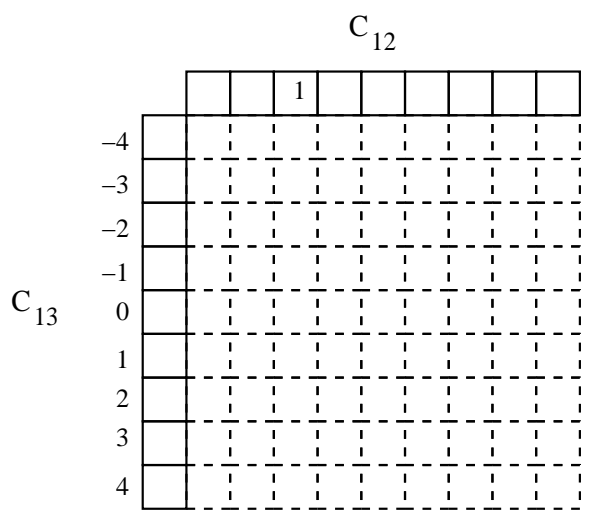

(a)

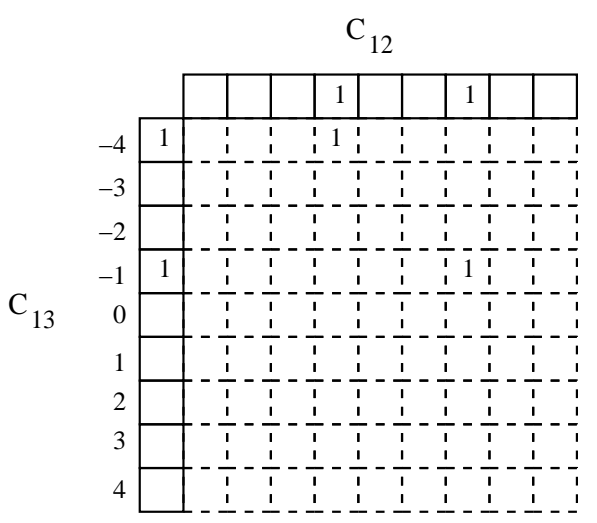

(b)

Figure 2: Single block correlations.

the blocks are tallied? The answer is only if $C_{12}$ and $C_{13}$ are independent. An example will demonstrate this property. Suppose the bin in channel 1 is chosen by the roll of a five-sided die, numbered 0 to 4 . The bins in channel 2 and 3 are then chosen by flipping a coin. One is added to the die for heads and one subtracted for tails. Correlations $C_{12}$ and $C_{13}$ are shown in Figure 3 normalized by the number of trials. These are the correlations whether the bin in channel 2 and 3 is chosen by a single flip for both or independent flips for each. The correlation $C_{123}$, however, changes depending on the independence of channel 2 and 3. These two cases are shown in Figure 3 and Figure 4. If channels 2 and 3 are chosen independently, $C_{123}$ is the outer product of $C_{12}$ and $C_{13}$ as shown in Figure 4 (b). If channels 2 and 3 are chosen by the same flip, $C_{123}$ is as shown in Figure 4.

Why don't the cross correlations in Figures 3 (a) and 4 (a) add up to one? This is the result of window bias which will be explained in the next paragraph. The deficiency in the 
probability comes from the combinations of a zero on the die along with a tails and a 4 on the die combined with a heads. In either case the result is not tallied.

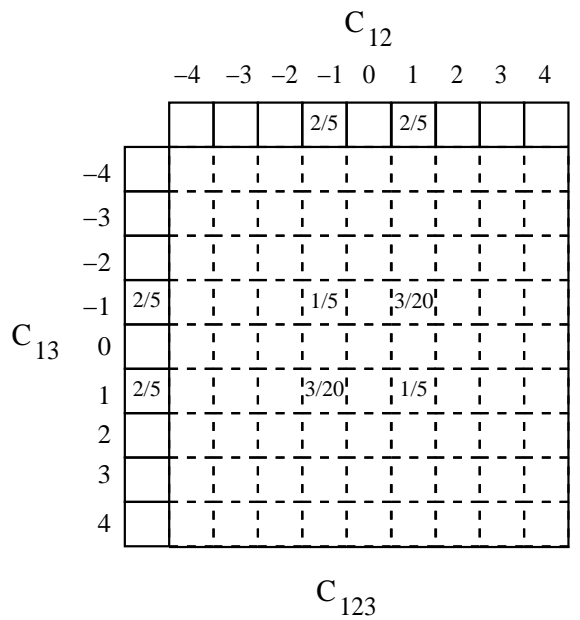

(a)

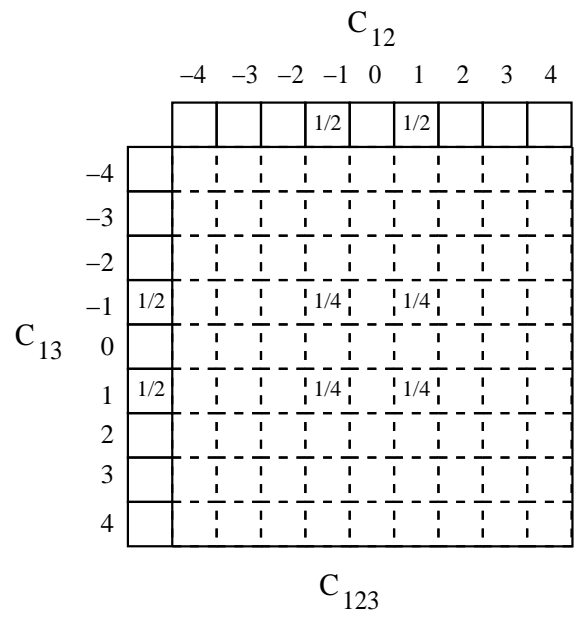

(b)

Figure 3: Independent correlation: (a) window biased and (b) unbiased.

Typically the ${ }^{252} C f$ source is input in channel 1 in NMIS. The blocks begin periodically. Blocks are not triggered on the events by NMIS. This approach causes a window bias in the cross-correlations. ${ }^{1} \quad$ The nature of the bias can be seen by returning to the dice examples. Suppose the bin in channel 1 is chosen by the throw of a die as in the preceding example. The bin in channel 2 is then chosen by throwing the same die and adding the value to that used for channel 1. If the number exceeds the number of bins it is discarded. The resulting normalized $C_{12}$ correlation is shown in Figure 5 (a). When bin one is chosen in channel 1 , delays of 1 to 4 are equally probable in channel 2 . When bin 2 is chosen, delays of 1,2 and 3 are equally likely in channel two, and so on. The window-biased cross correlation $C_{12}$ is shown in Figure 5 (a). If on the other hand we always assign the event in channel 1 to bin zero, $C_{12}$ there would be no window bias as shown in Figure 5 (b).

\footnotetext{
${ }^{1}$ For the algorithm to correct window bias in HOS correlations see[29] at page 130.
} 


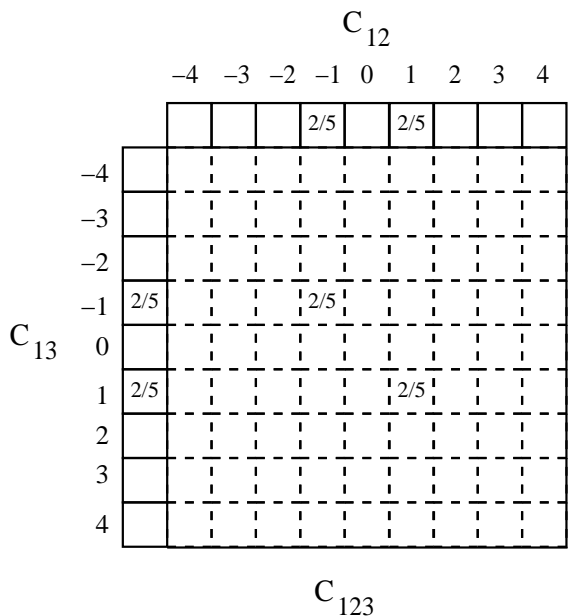

(a)

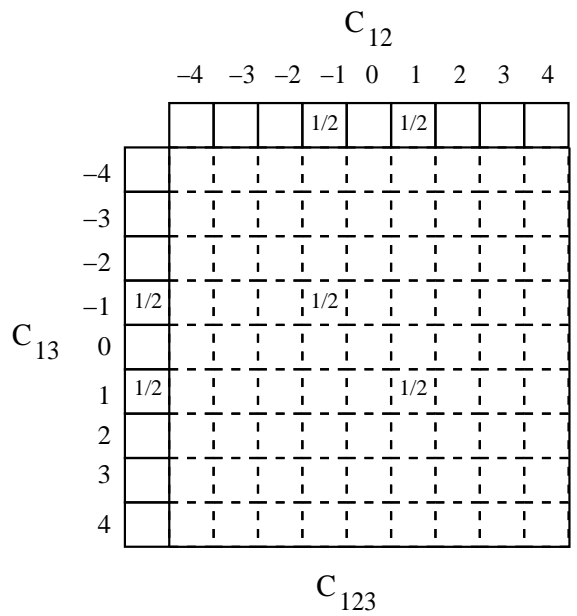

(b)

Figure 4: Dependent correlation: (a) window biased and (b) unbiased.

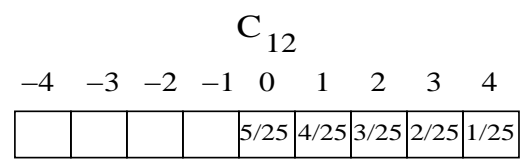

(a)

\begin{tabular}{|l|l|l|l|l|l|l|l|l|}
-4 & -3 & -2 & -1 & 0 & 1 & 2 & 3 & 4 \\
\hline & & & & $1 / 5$ & $1 / 5$ & $1 / 5$ & $1 / 5$ & $1 / 5$ \\
\hline
\end{tabular}

(b)

Figure 5: Window bias: (a) biased and (b) unbiased correlations.

\subsection{Definition of the correlation function}

The correlation of the functions $x_{1}\left(t_{1}\right)$ and $x_{2}\left(t_{2}\right)$ of the random variables $t_{1}$ and $t_{2}$ for a stationary process is defined as

$$
C_{12}(\tau)=E\left[x_{1}\left(t_{1}\right) x_{2}\left(t_{2}\right)\right]=E\left[x_{1}(t) x_{2}(t-\tau)\right] .
$$

For the time intervals of interest, the NMIS signals can be treated as stationary. That is, the correlation function depends only on the time difference between the signals, $\tau=t_{1}-t_{2}$. The random variables are the times $t_{1}$ and $t_{2}$ of detection events. The function $\mathrm{x}(\mathrm{t})$ is a tally of the number of detections in the interval $t, t+d t$. The expectation operator $E[x]$ is 
defined as

$$
E\left[x_{1}\left(t_{1}\right) x_{2}\left(t_{2}\right)\right]=\int_{-\infty}^{\infty} \int_{-\infty}^{\infty} x_{1} x_{2} p\left(x_{1}, x_{2}, t_{1}, t_{2}\right) d x_{1} d x_{2} d t_{1} d t_{2} .
$$

Assuming that the random variables $x_{1}$ and $x_{2}$ are independent of $t_{1}$ and $t_{2}$, the joint probability density can be factored so that

$$
\begin{aligned}
E\left[x_{1}\left(t_{1}\right) x_{2}\left(t_{2}\right)\right] & =\int_{-\infty}^{\infty} \int_{-\infty}^{\infty} x_{1} p\left(x_{1}\right) x_{2}, p\left(x_{2} \mid x_{1}\right) d x_{1} d x_{2} p\left(t_{1}, t_{2}\right) d t_{1} d t_{2} \\
& =\overline{x_{1} x_{2}} p\left(t_{1}, t_{2}\right) d t_{1} d t_{2} .
\end{aligned}
$$

The random variables $x_{1}$ and $x_{2}$ are counts, following binomial or Poisson probability distributions.

Focusing now on the time-delay dependent probability distribution, it can be factored and a delay variable substituted

$$
\begin{aligned}
p\left(t_{1}, t_{2}\right) d t_{1} d t_{2} & =p\left(t_{1}\right) p\left(t_{2} \mid t_{1}\right) d t_{1} d t_{2} \\
\tau & =t_{2}-t_{1} \\
d \tau & =d t_{2} \\
p(\tau) d \tau & =p\left(t_{1}\right) p\left(t_{1}+\tau \mid t_{1}\right) d t_{1} d \tau .
\end{aligned}
$$

Then integrating over the remaining time variable leaves $C_{12}(\tau)$ a function of the delay variable $\tau$ alone,

$$
\begin{aligned}
C_{12}(\tau) & =E\left[x_{1}(t) x_{2}(t-\tau)\right] \\
& =\overline{x_{1} x_{2}} \int_{-\infty}^{\infty} p\left(t_{1}\right) p\left(t_{1}+\tau \mid t_{1}\right) d t_{1} \\
& =\overline{x_{1} x_{2}} p(\tau) .
\end{aligned}
$$

\subsection{Correlation function in a thermal neutron system}

At this point an example will make clear how this analysis is applied to an actual experiment. Suppose we have a source which decays at random with an average rate $r_{1}$. The times of these decays will be designated by the random variable $t_{1}$. A detector will then detect the emission from this decay at a time $t_{2}$. The detection efficiency is unity. The emission is always detected. The multiplicity of the emission is also unity. The decay always results 
in one emission. Now suppose that the time of detection after emission, $\tau=t_{2}-t_{1}$, is a random variable with the probability density $p(\tau)=\frac{e^{-\frac{\tau}{\tau_{D}}}}{\tau_{D}}$. This probability density is typical of thermal neutron detectors such as those used in traditional multiplicity counting for NDA. The parameter $\tau_{D}$ is called the detector die-away time.

Equation 20 can be used in this example. Because $x_{1}\left(t_{1}\right)$ is random, there will be accidental correlations with $x_{2}\left(t_{2}\right)$. The accidental correlations occur at a rate of $r_{2}$ Because every emission is detected, the number of correlations $\overline{x_{1} x_{2}}$ over a measurement time of $t_{m}$ is $r_{1} t_{m}$.

From these, the correlation function is calculated:

$$
C_{12}(\tau)=r_{1} t_{m}\left(r_{2}+\frac{1}{\tau_{D}} e^{-\frac{\tau}{\tau_{D}}}\right)
$$

If detections are tallied in bins of width $\Delta t$ and we let $\tau=k \Delta t$, the correlation becomes

$$
\begin{aligned}
C_{12}(k) & =\int_{(k-1) \Delta t}^{k \Delta t} r_{1} t_{m}\left(r_{2}+\frac{1}{\tau_{D}} e^{-\frac{\tau}{\tau_{D}}}\right) d \tau \\
& =r_{1} t_{m}\left[r_{2} \Delta t+\left(1-e^{-\frac{\Delta t}{\tau_{D}}}\right) e^{-\frac{k \Delta t}{\tau_{D}}}\right] .
\end{aligned}
$$

The correlation $C_{12}(k)$ represents the expected number of detections accumulated in bin $k$ during a measurement time $t_{m}$. To demonstrate this result, the source-detector was simulated with 500 decays. The decay rate was set at $r_{1}=\frac{1}{100 \Delta t}$, and the detector die-away time was set at $\tau_{D}=4 \Delta t$. The results are shown in Figure 6 along with Equation 22 .

\subsection{Uncorrelated background and accidental correlations}

In the previous discussion because $x_{1}$ was a random process, the accidental rate $r_{1} r_{2}$ was included in the correlation. This accidental rate occurs even though the two channels were perfectly correlated. In the previous example, the source rate was 1 per 100 time bins on average. The detection rate was the same. The accidental count rate was therefore $r_{1} r_{2} \Delta t$ per time bin. These rates produced 5 counts per time bin for the 500 source counts in the example. If the source rate in this example is increased to 1 per 10 time bins and the measurement time decreased so that the total source counts is still 500 counts, the accidental count rate will be 50 counts per time bin as shown in Figure 7. 


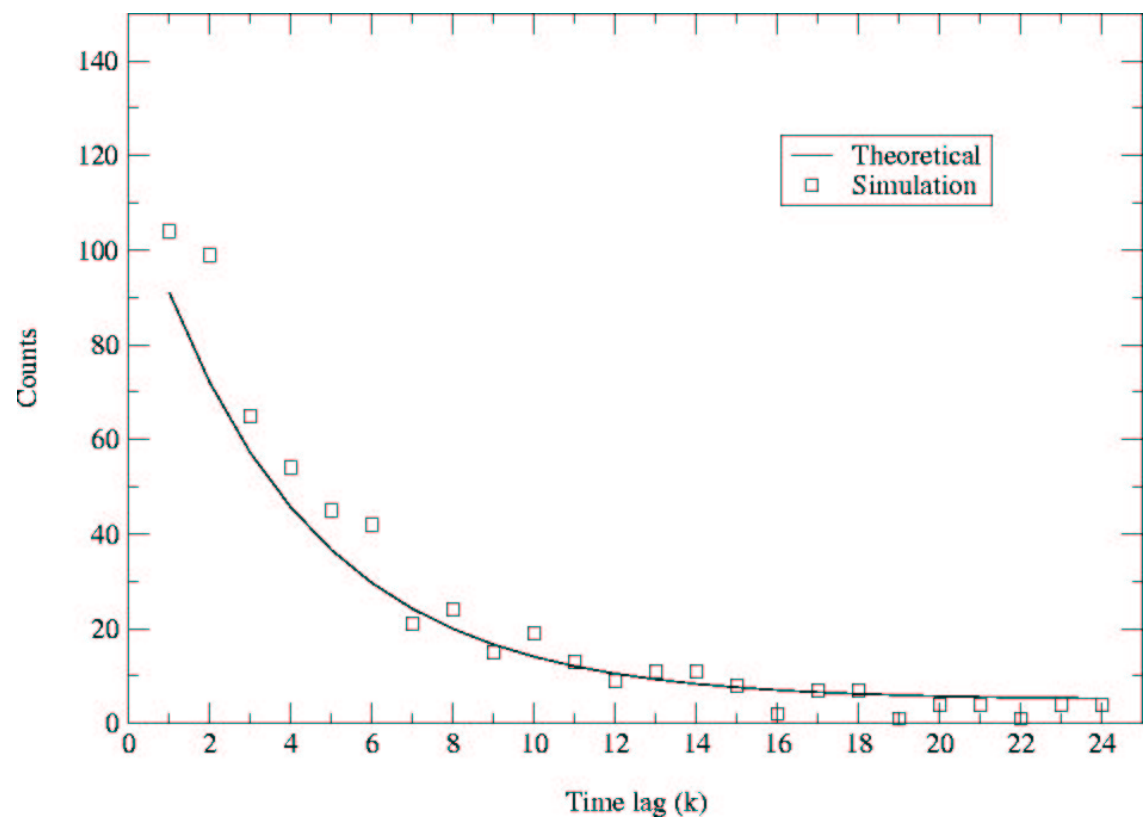

Figure 6: Source-detector correlations for a thermal detector for a source rate of $\frac{1}{100 \Delta t}$. The theoretical curve is from Equation (22).

Throughout most of this thesis it is assumed that the background and accidentals have been subtracted out. For bicorrelations, ${ }^{2}$ the accidentals include an accidental correlation with a correlated pair in addition to the uncorrelated triplet $r_{1} r_{2} r_{3}$.

$$
\begin{aligned}
C_{123 a c c i d e n t a l}\left(\tau_{12}, \tau_{13}\right) & =r_{1} C_{23}\left(\tau_{3}-\tau_{2}\right) \\
& +r_{2} C_{13}\left(\tau_{13}\right) \\
& +r_{3} C_{12}\left(\tau_{12}\right) \\
& +r_{1} r_{2} r_{3}
\end{aligned}
$$

It should be understood that the doubles correlations $C_{i j}$ in Equation 23 also have the background $r_{i} r_{j}$ subtracted out. In addition to accidental coincidences, uncorrelated background radiation is included in the accidentals in Equation 23.

\footnotetext{
${ }^{2} \mathrm{~A}$ more detailed treatment of the accidental coincidences and the relation to the covariance can be found in references $[29,30]$
} 


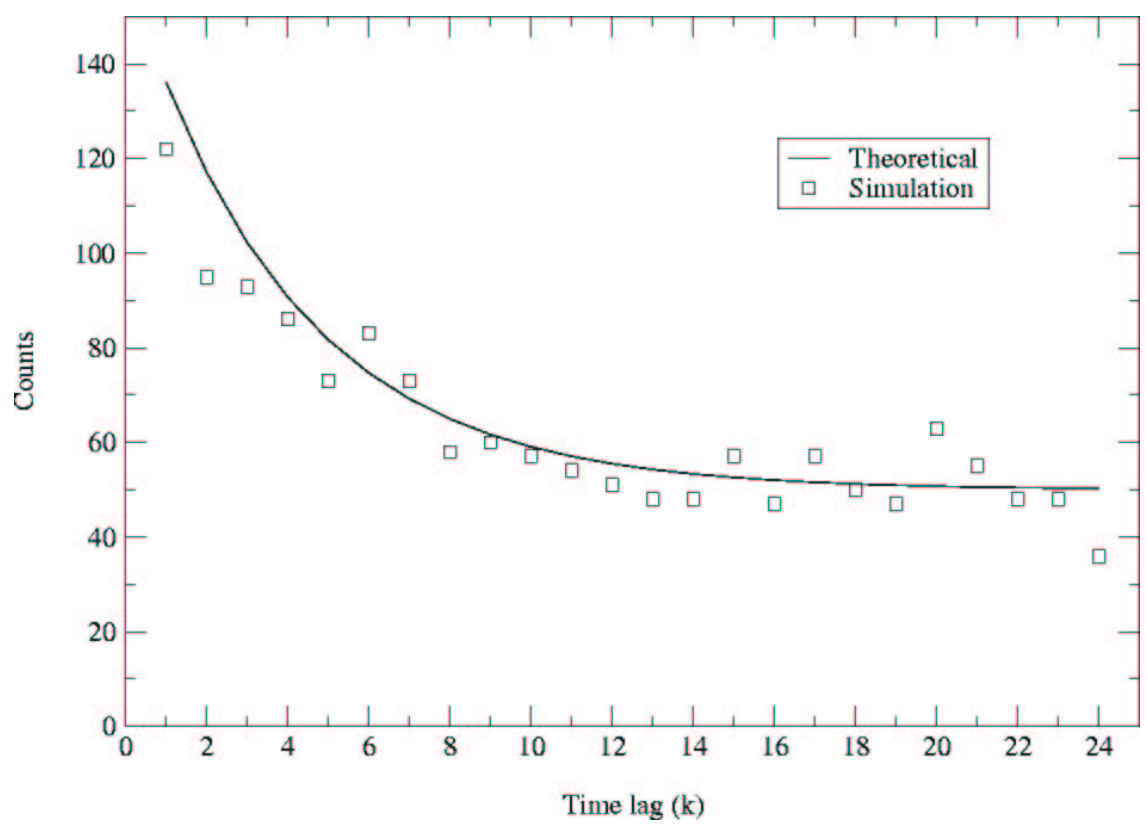

Figure 7: Source-detector correlations for a thermal detector for a source rate of $\frac{1}{10 \Delta t}$. The theoretical curve is from Equation (22).

\subsection{Correlation function in a fast neutron system}

In this final example the fast detectors used in this research are time correlated with a fission source. Assume an instrumented fission source has produced $N_{1}$ fissions during a measurement. Of these $\epsilon_{f}$ are detected in Channel 1. Each fission will produce $\bar{\gamma}$ gamma rays and $\bar{\nu}$ neutrons on average. These are of course the prompt radiations from fission and will be used that way throughout. The probability of detecting the gamma rays and neutrons is $\epsilon_{g}$ and $\epsilon_{n}$ respectively. The total number of fission-gamma and fission-neutron pairs will then be $\epsilon_{f} N_{1} \bar{\gamma} \epsilon_{g}$ and $\epsilon_{f} N_{1} \bar{\nu} \epsilon_{n}$ respectively.

The gamma rays will all arrive at the detector at nearly the same time. We can therefore represent the time distribution of this arrival time as

$$
p_{g}\left(\tau_{12}\right)=\delta\left(\tau_{12}-\frac{d_{2}}{c}\right)
$$

where $d_{2}$ is the distance between the fission source and the detector, $\tau_{12}$ is the difference between the time of detection $t_{2}$ and the time of the fission at $t_{1}$ and $c$ is the speed of light. The $\delta$ can be taken either literally or at least implying a narrow probability distribution. 
The neutron fission spectrum is a function of energy $\chi(E)$. The energy of a neutron is related to its velocity by

$$
E=\frac{1}{2} m v^{2}=\frac{1}{2} m\left(\frac{d_{2}}{\tau_{12}}\right)^{2} .
$$

The relationship between the spectrum as a function of time and the spectrum as a function of energy is $\chi(\tau) d \tau=\chi(E) d E$ where $\frac{d E}{d \tau}=\frac{E}{\tau}$. Therefore the time distribution of neutron detections subsequent to a fission can be written

$$
p_{n}\left(\tau_{12}\right)=\epsilon_{n}\left(\tau_{12}\right) \chi\left(\tau_{12}\right)=\epsilon_{n}\left(\tau_{12}\right) \chi\left(E \rightarrow \frac{1}{2} m\left(\frac{d_{2}}{\tau_{12}}\right)^{2}\right) \frac{E}{\tau_{12}} .
$$

Substituting into Equation 20 results in

$$
C_{12}\left(\tau_{12}\right)=\epsilon_{f} N_{1} \bar{\gamma} \epsilon_{g} \delta\left(\tau_{12}-\frac{d_{2}}{c}\right)+\epsilon_{f} N_{1} \bar{\nu} \epsilon_{n}\left(\tau_{12}\right) \chi\left(\tau_{12}\right) .
$$

A measurement of $C_{12}\left(\tau_{12}\right)$ is shown in Figure 8. Other correlations can be derived from this correlation as will be described in the next chapter.

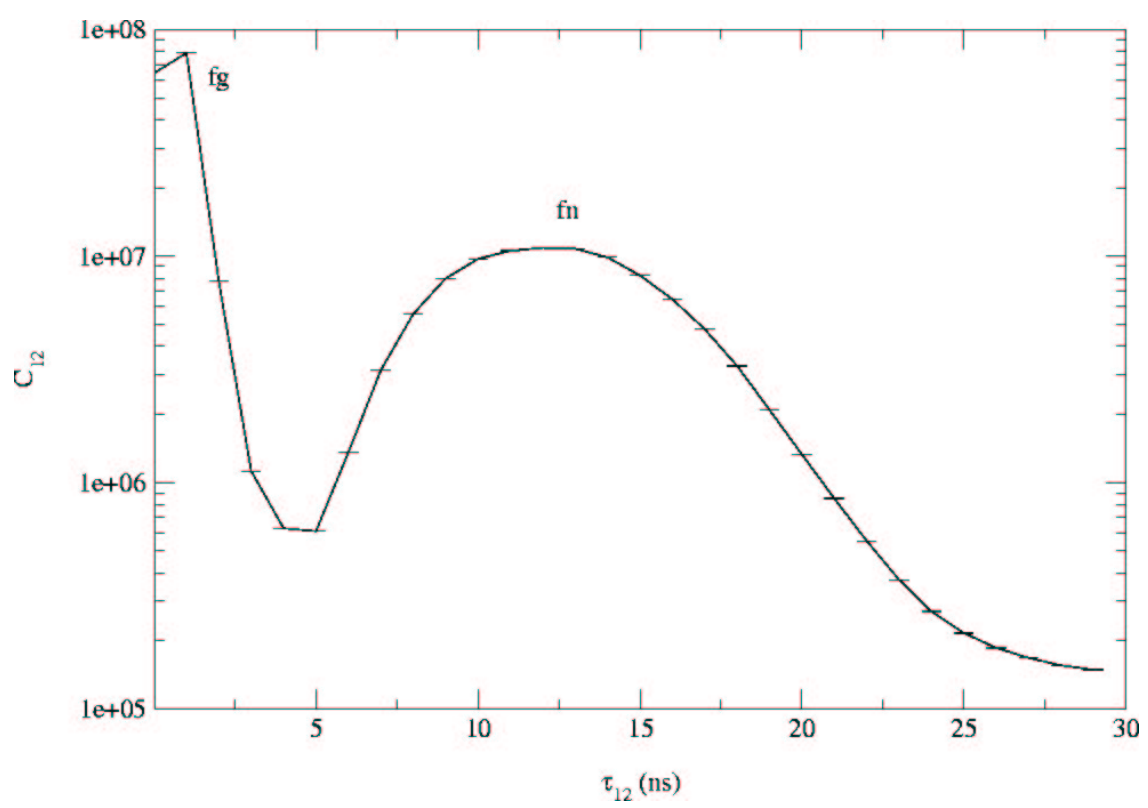

Figure 8: Measurement of $C_{12}\left(\tau_{12}\right)$ in total correlated counts for a 3 -inch detector from the experiment as described in Chapter 7 . 


\subsubsection{Detector efficiency in a fast neutron detector}

The most important factor affecting efficiency in these experiments is the detector geometry factor. The geometry factor can be written as $g=\frac{\Omega}{4 \pi}$. Knoll provides an expression for $\Omega$ for a right circular cylinder. ${ }^{3} \quad$ The cylindrical formula works reasonably well even for a rectangular detector. The geometry factor for the 3 -inch detector at the $23 \mathrm{~cm}$ distance is $8.5 \times 10^{-3}$ and $1.5 \times 10^{-2}$ for the 4 -inch detector. The intrinsic efficiency can also be considered. The most important feature of the detector intrinsic efficiency is the threshold. The detectors are virtually blind to neutrons below the threshold of $1 \mathrm{MeV}$. The intrinsic efficiency of the detectors can be measured.

The detection efficiency of a detector can be measured from the source detector correlation $C_{12}(\tau)$, provided that there is a reasonable distance between the source and detector, and there is no intervening material.[33] The source detector correlation can be converted to a function of energy by the relation $E=\frac{1}{2} m\left(\frac{d}{\tau}\right)^{2}$. The correlation $C_{12}(E)$ represents the number of neutrons in the energy range $(E, E+d E)$ detected per fission. The number of neutrons in that energy range impinging on the detector can also be predicted. This number is $N(E) d E=\bar{\nu} g \chi(E) d E$ per ${ }^{252} C f$ fission. The efficiency is then ${ }^{4}$

$$
\epsilon_{n}(E)=\frac{C_{12}(E)}{N(E)}
$$

The correlation $C_{12}(\tau)$ is actually integrated over the range from $\tau$ to $\tau+\Delta \tau$. Although $\Delta \tau$ is a constant, $\Delta E$ is not. The boundaries of the energy range, $E_{i}, E_{i+1}$ must therefore be integrated over to arrive at the predicted number of neutrons. It then becomes $N(E)=\bar{\nu} g \int_{E_{i}}^{E_{i+1}} \chi(E) d E$. The average number of neutrons emitted per ${ }^{252} C f$ fission is designated as $\bar{\nu}$.

An efficiency computed from Equation (28) is shown in Figure 9. Below the threshold of about $1 \mathrm{MeV}$, no neutrons are detected. The detection efficiency quickly rises to a

\footnotetext{
${ }^{3}$ The formula from Reference [24] at page 119 is given in Appendix B.6. A formula for a rectangular detector form Reference [19] is also given in that appendix.

${ }^{4}$ This formula assumes that there is a one-to-one correspondence between energy and time of detection. See reference [11] for an alternative formula.
} 


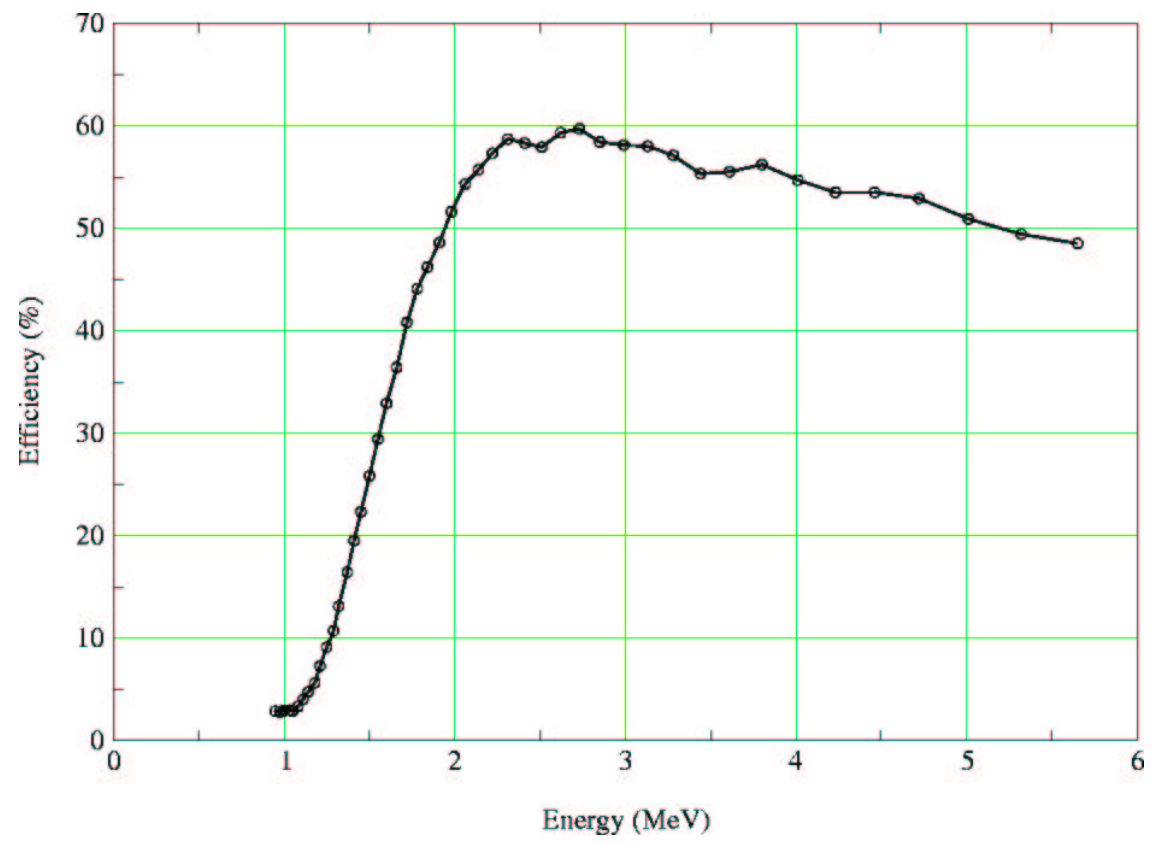

Figure 9: Intrinsic neutron detection efficiency computed from a time-of-flight measurement at $1 \mathrm{~m}$.

peak at about $2.5 \mathrm{MeV}$. As the neutron energy increases above the threshold the probability that it deposits enough energy to exceed the threshold increases. At energies above $2.5 \mathrm{MeV}$, the efficiency gradually declines as the neutron energy increases. This decline reflects the increasing probability that higher energy neutrons pass through the detector without interacting. There are alternative models for calculating detection efficiency.[11]

The neutron detection efficiency $\epsilon_{n}$ is a function of $\tau$ as long as the relation between $E_{n}$ and $\tau$ holds. The integral

$$
\epsilon_{n}=\frac{\int_{0}^{\infty} \epsilon_{n}\left(\tau_{12}\right) \chi\left(\tau_{12}\right) d \tau_{12}}{\int_{0}^{\infty} \chi\left(\tau_{12}\right) d \tau_{12}}
$$

is the average detection efficiency for fission neutrons. This average is used in traditional shift register analysis and will be employed in this research where a time variable is integrated out.

\subsection{Error Analysis}

An estimate of the correlation function $C_{i j}(k)$ in time bin $k$ is computed by subtracting the background $r_{i} r_{j}$ from the total count data $R_{i j}(k)$ and correcting for the window bias with 
the factor $W(k)$. This estimate can be written

$$
C_{i j}(k)=R_{i j}(k) W(k)-r_{i} r_{j}
$$

The background $r_{i} r_{j}$ is the product of the count rates per bin in each of the two detectors, $r_{i}=\frac{C_{i i}(0)}{T}$ where $T$ is the total number of time bins in the entire measurement time. The window bias factor is $W(k)=\frac{N}{N-k}$. The uncertainty in the estimate is then

$$
\begin{aligned}
\sigma_{C_{i j}(k)} & =\sqrt{W^{2}(k) R_{i j}(k)+r_{i} r_{j}\left(r_{i}+r_{j}\right)(\Delta t)^{2} T} \\
& \approx W(k) \sqrt{R_{i j}(k)} \\
& \approx \sqrt{C_{i j}(k)+r_{i} r_{j}(\Delta t)^{2}} .
\end{aligned}
$$

The background contribution to the uncertainty can be neglected. The background rate is $7 \times 10^{-5}$ and $2 \times 10^{-5}$ per nanosecond in the source and 3 -inch detectors respectively. This produces a background rate in the source-detector cross correlation of 0.14 counts per second per time bin. The count rate in the neutron peak at $12 \mathrm{~ns}$, on the other hand, is 80 counts per second per time bin. The total uncertainty in the neutron peak is then $\sigma_{C_{12}(12)}=1.05\left(80 s^{-1}+1.2 \times 10^{-4} s^{-1}\right) T$. The background contribution to the uncertainty of $1.2 \times 10^{-4} \mathrm{~s}^{-1}$ can clearly be neglected.

In the case of the bicorrelation, the uncertainty can be approximated as

$$
\begin{aligned}
\sigma_{C(m n)} & \approx \sqrt{W^{2}(m n) R(m n)+r_{i}^{2} \sigma_{C(m-n)}^{2}+r_{j}^{2} \sigma_{C(n)}^{2}+r_{k}^{2} \sigma_{C(m)}^{2}} \\
& \approx W(m n) \sqrt{R(m n)} .
\end{aligned}
$$

The approximation indicates that the error in the $r_{i} r_{j} r_{k}$ term was neglected because it is generally small. The window bias factor can also be neglected as long as the time bin $k$ is small compared to $N$ which is 512 .

Marginal correlations are frequently computed in the analysis by integrating over a time variable. An example would be

$$
\begin{aligned}
C_{j k \mid i}\left(\tau_{j k}\right) & =\int_{0}^{\infty} C_{i j k}\left(\tau_{j}, \tau_{j k}+\tau_{j}\right) d \tau_{j} \\
C_{j k \mid i}(n) & =\sum_{l=0}^{\infty} C_{i j k}(l, n+l)
\end{aligned}
$$


where $\tau_{k}=\tau_{j k}+\tau_{j}$. The variance in $C_{j k \mid i}\left(\tau_{j k}\right)$ is then

$$
\begin{aligned}
\sigma_{j k \mid i}^{2}\left(\tau_{j k}\right) & \approx \int_{0}^{\infty} W^{2}\left(\tau_{j}, \tau_{j k}+\tau_{j}\right) R_{i j k}\left(\tau_{j}, \tau_{j k}+\tau_{j}\right) d \tau_{j} \\
& \approx \int_{0}^{\infty} R_{i j k}\left(\tau_{j}, \tau_{j k}+\tau_{j}\right) d \tau_{j} .
\end{aligned}
$$

The window bias can be ignored for delay times which are much less than the $512 \mathrm{~ns}$ window. 


\section{CHAPTER IV}

\section{ANALYTICAL EXPRESSIONS AND TEMPORAL SEPARATION OF NEUTRON AND GAMMA COINCIDENCES FROM FISSION AND OTHER SOURCES}

This chapter develops a point model for the time dependence of the fission detection process by fast plastic detectors. In this case, "point" refers to a spontaneous fission source localized in space rather than a point reactor which is distributed in space. This chapter will first show the relationship between the source-detector correlation $C_{12}\left(\tau_{12}\right)$, the sourcedetector-detector bicorrelation $C_{123}\left(\tau_{12}, \tau_{13}\right)$, the detector-detector correlation $C_{23}\left(\tau_{23}\right)$ and the three-detector bicorrelation $C_{234}\left(\tau_{23}, \tau_{24}\right)$ from a spontaneous fission source.

The purpose of the expressions for the correlations is to predict the temporal regions in which various combinations of detections occur and to show the relationship between detection pairs and triples for these combinations. The region on $\tau_{12}$ in which fissiongamma $(f g)$ detection pairs and fission-neutron $(f n)$ detection pairs will be evident from the expressions. The regions in the $\tau_{12}, \tau_{13}$ in which $f g g, f g n, f n g$ and $f n n$ combinations occur can be predicted from $C_{123}\left(\tau_{12}, \tau_{13}\right)$. Similarly the region in the $\tau_{23}$ in which $g g, g n, n g$ and $n n$ combinations occur will be evident from the expression of $C_{23}\left(\tau_{23}\right)$. It is not necessary to know the exact time distribution of the correlations. It is only necessary to know that it is bounded by the detection of the most energetic neutrons and the detection threshold set in the constant fraction discriminator. Therefore, an expression for $\epsilon_{n}\left(\tau_{12}\right) \chi\left(\tau_{12}\right)$ will not be specified. The $f g$ detection distribution, $\epsilon_{g} \delta\left(\tau_{12}-\frac{d_{2}}{c}\right)$, is only specified to be a narrow distribution.

The relationship between detection pairs and triples for combinations of gamma rays and 
neutrons will then be analyzed in Sections 4.2 and 4.3 where various ratios will be presented. From these ratios, detection efficiency and ultimately the number of spontaneous fissions can be determined. The method and data to calculate the multiplicity moments will be described in Section 4.4 for both neutrons and gamma rays.

In Section 4.5 the factors affecting the exact time distribution of the correlations will be discussed. For a fast proton-recoil detection system, these factors include the spatial distribution of the fissile material,[9] the spatial distribution of scattering material as well as the multiplication of the fissile material.[31] Unless all but one of these factors are held constant, it futile to use the time distribution to determine these factors. For example, to determine the spatial distribution of a spontaneous fission source, Chiang restricted the distribution to non-multiplying configurations.[9]

Finally, non-fission reactions such as nuclear decay, scattering, and alpha-n reactions produce correlated detections. These correlations will be described in Section 4.6.

\section{$\underline{4.1 \quad \text { Source and detector correlations from a point fission source }}$}

The first correlation to be considered is between the instrumented source and a detector separated by a distance $d_{2}$ in air, i.e., there is no intervening material. A mathematical expression for this correlation was shown from Equation 27 to be

$$
\begin{aligned}
C_{12}\left(\tau_{12}\right) & =N_{1} p\left(\tau_{12}\right) \\
& =N_{1} \epsilon_{f}\left[\bar{\gamma} \epsilon_{g} \delta\left(\tau_{12}-\frac{d_{2}}{c}\right)+\bar{\nu} \epsilon_{n}\left(\tau_{12}\right) \chi\left(\tau_{12}\right)\right] .
\end{aligned}
$$

All of the uncorrelated background has been subtracted from $C_{12} . N_{1}$ represents the number of fissions occurring in the instrumented source, and $\epsilon_{f}$ represents the probability of detecting the fissions in the instrumented source.

It is convenient in the development of the analysis to rewrite the correlation function as $C_{12}\left(\tau_{12}\right)=C_{f g}\left(\tau_{12}\right)+C_{f n}\left(\tau_{12}\right)$ where

$$
\begin{array}{lr}
C_{f g}\left(\tau_{12}\right)=N_{1} \epsilon_{f} \bar{\gamma} \epsilon_{g} \delta\left(\tau_{12}-\frac{d}{c}\right) & \text { represents the gamma response, and } \\
C_{f n}\left(\tau_{12}\right)=N_{1} \epsilon_{f} \bar{\nu} \epsilon_{n}\left(\tau_{12}\right) \chi\left(\tau_{12}\right) & \text { represents the neutron response. }
\end{array}
$$


A measurement of this function is shown in Figure 10 on log scale. ${ }^{1}$ This measurement is taken from the source-detector setup described in Chapter 7. The source-to-detector distance $d_{2}$ is $23 \mathrm{~cm}$. The gamma response $C_{f g}\left(\tau_{12}\right)$ can clearly be seen at $\frac{d}{c}=0.8 \mathrm{~ns}$. It has a width of $\Delta_{g} \approx 2$ ns. The neutron spectrum $C_{f n}\left(\tau_{12}\right)$ is temporally separated from the gamma peak. It extends from the time the fastest neutrons $n_{f}$ are detected $\tau_{f}=\frac{d}{v_{f}}$ to the time that the threshold energy neutrons $n_{t}$ are detected $\tau_{t}=\frac{d}{v_{t}}$. The threshold is typically $1 \mathrm{MeV}$ corresponding to neutrons which have a velocity of $1.4 \mathrm{~cm} / \mathrm{ns}$. The fast neutrons have a velocity of about $4.8 \mathrm{~cm} / \mathrm{ns}$. This velocity is observed from Figure 10. It ultimately comes from a combination of the Maxwellian distribution and detection efficiency.

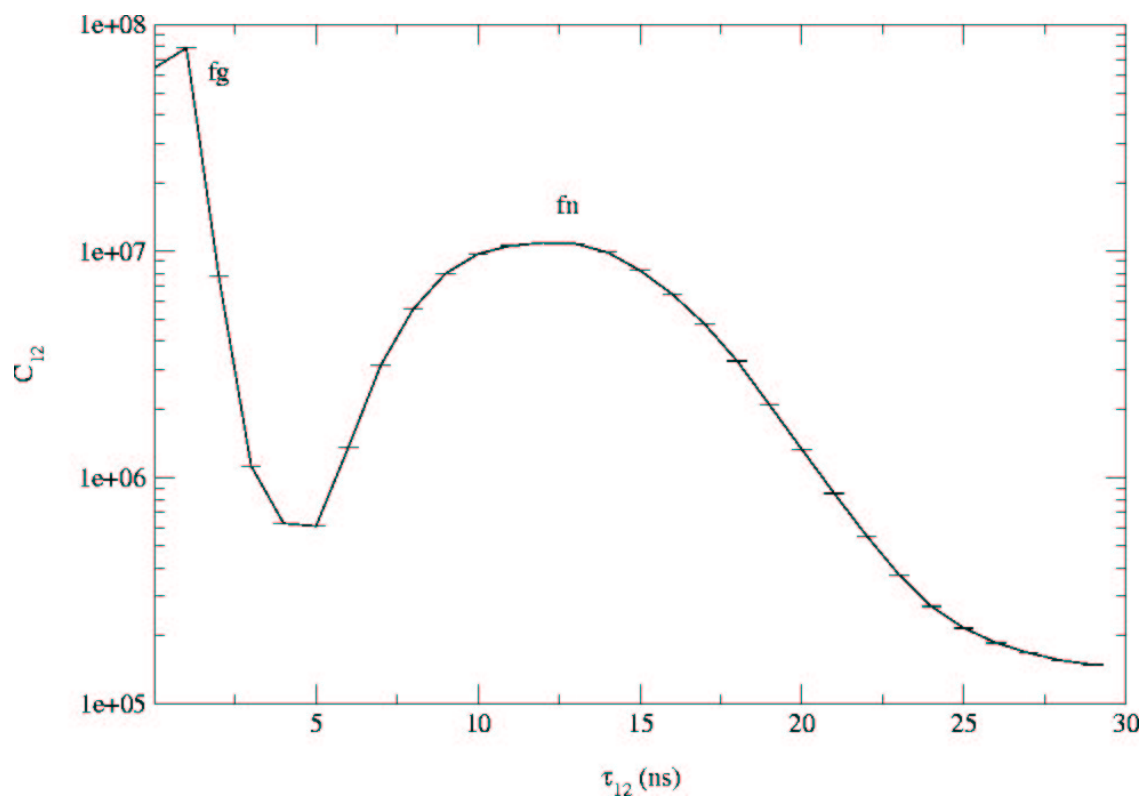

Figure 10: Measurement of $C_{12}\left(\tau_{12}\right)$ in total correlated counts for a 3 -inch detector from the experiment as described in Chapter 7.

The instrumented source can also be correlated with two detectors. This correlation is shown in Figure 11 where the source-to-detector distance $d_{2}$ and $d_{3}$ was $23 \mathrm{~cm}$ for each

\footnotetext{
${ }^{1}$ This correlation is also plotted on linear scale in Figure 40. For comparison, a purely theoretical version of this correlation can also be seen in Figure 23.
} 
detector. The correlation function can be written as

$$
C_{123}\left(\tau_{12}, \tau_{13}\right)=C_{f g g}\left(\tau_{12}, \tau_{13}\right)+C_{f g n}\left(\tau_{12}, \tau_{13}\right)+C_{f n g}\left(\tau_{12}, \tau_{13}\right)+C_{f n n}\left(\tau_{12}, \tau_{13}\right) .
$$

Recalling the definition of a correlation from Section 3.2 and assuming the dependence in the probability of a detection can be factored from the time variable, each term in Equation (36) can be written

$$
\begin{aligned}
& C_{f g g}\left(\tau_{12}, \tau_{13}\right)=N_{1} \epsilon_{f} \overline{\gamma(\gamma-1)} \epsilon_{g}^{2} \delta\left(\tau_{12}-\frac{d_{2}}{c}\right) \delta\left(\tau_{13}-\frac{d_{3}}{c}\right) \\
& C_{f g n}\left(\tau_{12}, \tau_{13}\right)=N_{1} \epsilon_{f} \overline{\nu \gamma} \epsilon_{g} \delta\left(\tau_{12}-\frac{d_{2}}{c}\right) \epsilon_{n}\left(\tau_{13}\right) \chi\left(\tau_{13}\right) \\
& C_{f n g}\left(\tau_{12}, \tau_{13}\right)=N_{1} \epsilon_{f} \overline{\nu \gamma} \epsilon_{g} \epsilon_{n}\left(\tau_{12}\right) \chi\left(\tau_{12}\right) \delta\left(\tau_{13}-\frac{d_{13}}{c}\right) \\
& C_{f n n}\left(\tau_{12}, \tau_{13}\right)=N_{1} \epsilon_{f} \overline{\nu(\nu-1)} \epsilon_{n}\left(\tau_{12}\right) \chi\left(\tau_{12}\right) \epsilon_{n}\left(\tau_{13}\right) \chi\left(\tau_{13}\right) .
\end{aligned}
$$

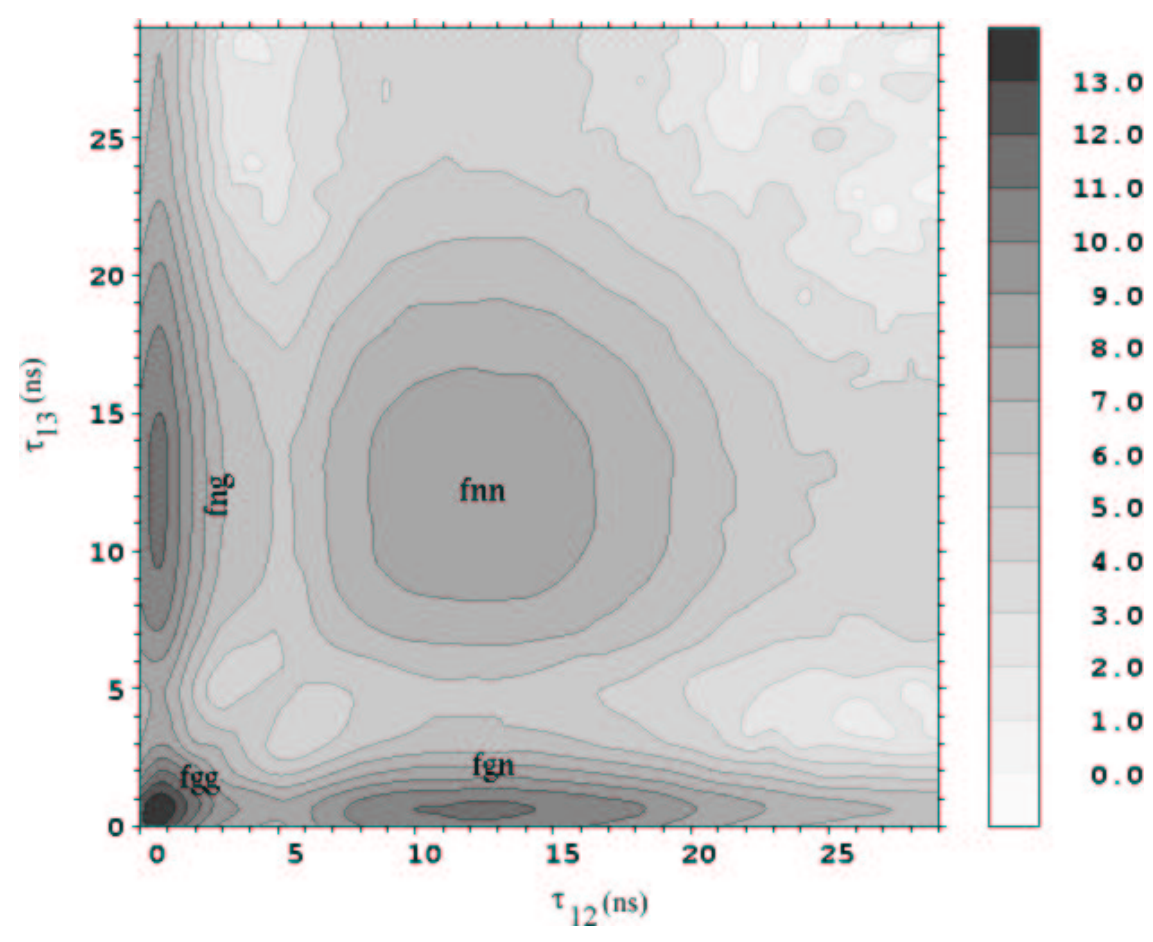

Figure 11: Measurement of $C_{123}\left(\tau_{12}, \tau_{13}\right)$ in $\log$ of total correlated counts. The source-to-detector distance is $23 \mathrm{~cm}$ for both detectors 2 and 3 . 
It is assumed that the only dependence between the correlations is the reduction in the number of the available radiations after the other detection. The reduction in the number of radiations is only true if the detectors are separated by a considerable distance. It is not improbable for a single radiation to register in two adjacent detectors.[43]

The bicorrelation $C_{123}\left(\tau_{12}, \tau_{13}\right)$ calculated from the individual terms is shown in Figure 12. A graph of the equation for the substitution of variables $\tau_{23}=\tau_{13} \tau_{12}$ is shown in the figure. Of course that graph represents the family of graphs for all $\tau_{23}$. The assumption that the time distribution of the two correlations are independent turns out to be reasonably correct. In other words, if a fast neutron is detected in one detector, the neutron detected in the second detector is no less likely to be fast or slow.

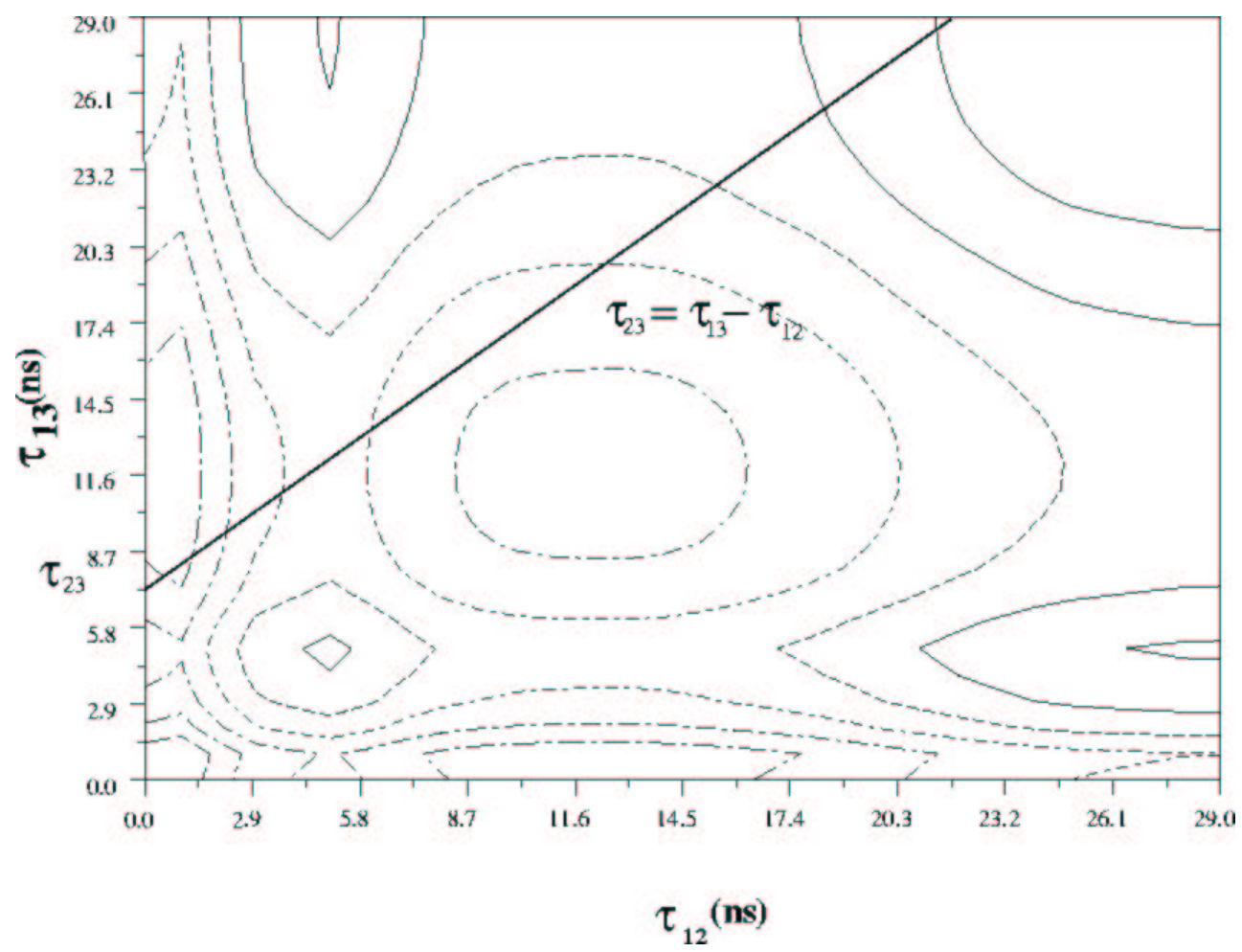

Figure 12: $C_{12}\left(\tau_{12}\right) C_{13}\left(\tau_{13}\right)$ with the substitution of variables $\tau_{23}=\tau_{13}-\tau_{12}$.

Each of these terms can be seen in the actual measured bicorrelation in Figure 11. Each term is temporally separated from the others in the $\tau_{12}, \tau_{13}$ plane. Each of the four regions can be defined by rectangular bounds as defined in Table 3. The fissions correlated with 
gamma-gamma pairs form essentially a point at $\tau_{12}=\frac{d_{2}}{c}$ and $\tau_{13}=\frac{d_{3}}{c}$ with a width of $\Delta_{g}$. The fissions correlated with gamma-neutron pairs form lines parallel to the $\tau_{12}$ and $\tau_{13}$ axes and a width of $\Delta_{g}$. The beginning and end of these lines are marked by fast $n_{f}$ and threshold $n_{t}$ neutrons respectively. The fission-neutron-neutron triplets are bounded by a rectangle formed from the delay times of each of the four pairs of fast and threshold neutrons.

Table 3: Temporal bounds for $C_{123}\left(\tau_{12}, \tau_{13}\right)$ and $C_{23}\left(\tau_{23}\right)$

\begin{tabular}{|lc|cc|c|}
\hline Correlation & Combination & $\tau_{12}$ & $\tau_{13}$ & $\tau_{23}$ \\
\hline$C_{f g g}$ & $f g g$ & $\frac{d_{2}}{c} \pm \frac{\Delta_{g}}{2}$ & $\frac{d_{3}}{c} \pm \frac{\Delta_{g}}{2}$ & $\frac{d_{3}-d_{2}}{c} \pm \frac{\Delta_{g}}{2}$ \\
\hline$C_{f g n}$ & $f g n_{f}$ & $\frac{d_{2}}{c} \pm \frac{\Delta_{g}}{2}$ & $\frac{d_{3}}{v_{f}}$ & $\frac{d_{3}}{v_{f}}-\frac{d_{2}}{c}$ \\
& $f g n_{t}$ & $\frac{d_{2}}{c} \pm \frac{\Delta_{g}}{2}$ & $\frac{d_{3}}{v_{t}}$ & $\frac{d_{3}}{v_{t}}-\frac{d_{2}}{c}$ \\
\hline$C_{f n g}$ & $f n_{f} g$ & $\frac{d_{2}}{v_{f}}$ & $\frac{d_{3}}{c} \pm \frac{\Delta_{g}}{2}$ & $\frac{d_{3}}{c}-\frac{d_{2}}{v_{f}}$ \\
& $f n_{t} g$ & $\frac{d_{2}}{v_{t}}$ & $\frac{d_{3}}{c} \pm \frac{\Delta_{g}}{2}$ & $\frac{d_{3}}{c}-\frac{d_{2}}{v_{t}}$ \\
\hline \multirow{3}{*}{$C_{f n n}$} & $f n_{f} n_{t}$ & $\frac{d_{2}}{v_{f}}$ & $\frac{d_{3}}{v_{t}}$ & $\frac{d_{3}}{v_{t}}-\frac{d_{2}}{v_{f}}$ \\
& $f n_{f} n_{f}$ & $\frac{d_{2}}{v_{f}}$ & $\frac{d_{3}}{v_{f}}$ & - \\
& $f n_{t} n_{t}$ & $\frac{d_{2}}{v_{t}}$ & $\frac{d_{3}}{v_{t}}$ & - \\
& $f n_{t} n_{f}$ & $\frac{d_{2}}{v_{t}}$ & $\frac{d_{3}}{v_{f}}$ & $\frac{d_{3}}{v_{f}}-\frac{d_{2}}{v_{t}}$ \\
\hline
\end{tabular}

A cross correlation $C_{23}$ between two detectors is shown in Figure 13 on log scale. ${ }^{2} \quad \mathrm{~A}$ related detector-detector correlation $C_{23 \mid 1}$ can be derived from the source-detector-detector correlation by a substitution of variables $\tau_{23}=\tau_{13}-\tau_{12}$ as shown in Figure 12. This correlation is conditional on a fission being detected in Channel 1. The boundaries of the four regions of this correlation are determined from this substitution of variables and also shown in Table 3. This correlation can be thought of as the shadow of the bicorrelation $C_{123}$ projected on the $\tau_{13}$ axis.

\footnotetext{
${ }^{2}$ This correlation is also plotted on linear scale in Figure 41.
} 


$$
\begin{aligned}
C_{23 \mid 1}\left(\tau_{23}\right) & =\int_{0}^{\infty} C_{123}\left(\tau_{12}, \tau_{23}+\tau_{12}\right) d \tau_{12} \\
& =C_{g g \mid f_{1}}\left(\tau_{23}\right)+C_{g n \mid f_{1}}\left(\tau_{23}\right)+C_{n g \mid f_{1}}\left(\tau_{23}\right)+C_{n n \mid f_{1}}\left(\tau_{23}\right)
\end{aligned}
$$

where

$$
\begin{aligned}
C_{g g \mid f_{1}}\left(\tau_{23}\right) & =N_{1} \epsilon_{f} \overline{\gamma(\gamma-1)} \epsilon_{g}^{2} \int_{0}^{\infty} \delta\left(\tau_{12}-\frac{d_{2}}{c}\right) \delta\left(\tau_{23}+\tau_{12}-\frac{d_{13}}{c}\right) d \tau_{12} \\
& =N_{1} \epsilon_{f} \overline{\gamma(\gamma-1)} \epsilon_{g}^{2} \delta\left(\tau_{23}-\frac{d_{3}-d_{2}}{c}\right) \\
C_{g n \mid f_{1}}\left(\tau_{23}\right) & =N_{1} \epsilon_{f} \overline{\nu \gamma} \epsilon_{g} \int_{0}^{\infty} \delta\left(\tau_{12}-\frac{d_{2}}{c}\right) \epsilon_{n}\left(\tau_{23}+\tau_{12}\right) \chi\left(\tau_{23}+\tau_{12}\right) d \tau_{12} \\
& =N_{1} \epsilon_{f} \overline{\nu \gamma} \epsilon_{g} \epsilon_{n}\left(\tau_{23}-\frac{d_{2}}{c}\right) \chi\left(\tau_{23}-\frac{d_{2}}{c}\right) \\
C_{n g \mid f_{1}}\left(\tau_{23}\right) & =N_{1} \epsilon_{f} \overline{\nu \gamma} \epsilon_{g} \int_{0}^{\infty} \epsilon_{n}\left(\tau_{12}\right) \chi\left(\tau_{12}\right) \delta\left(\tau_{23}+\tau_{12}-\frac{d_{3}}{c}\right) d \tau_{12} \\
& =N_{1} \epsilon_{f} \overline{\nu \gamma} \epsilon_{g} \epsilon_{n}\left(\frac{d_{3}}{c}-\tau_{23}\right) \chi\left(\frac{d_{3}}{c}-\tau_{23}\right) \\
C_{n n \mid f_{1}}\left(\tau_{23}\right) & =N_{1} \epsilon_{f} \overline{\nu(\nu-1)} \int_{0}^{\infty} \epsilon_{n}\left(\tau_{12}\right) \chi\left(\tau_{12}\right) \epsilon_{n}\left(\tau_{23}+\tau_{12}\right) \chi\left(\tau_{23}+\tau_{12}\right) d \tau_{12}
\end{aligned}
$$

The only difference in the case of the cross correlation $C_{23}$ is that the fissions need not be detected. This difference can be designated by substituting $N$ for $N_{1}$ and setting $\epsilon_{f}$ to one. This implies that $\frac{C_{23 \mid 1}}{C_{23}}=\frac{N_{1} \epsilon_{f}}{N}$. In addition, if the observed fission source $N_{1}$ is the only fission source then $N_{1}=N$.

Finally, a correlation between three detectors can be measured. Such a correlation is shown in Figure 14. Again the source-to-detector distance for all three detectors $\left(d_{1}, d_{2}\right.$ 


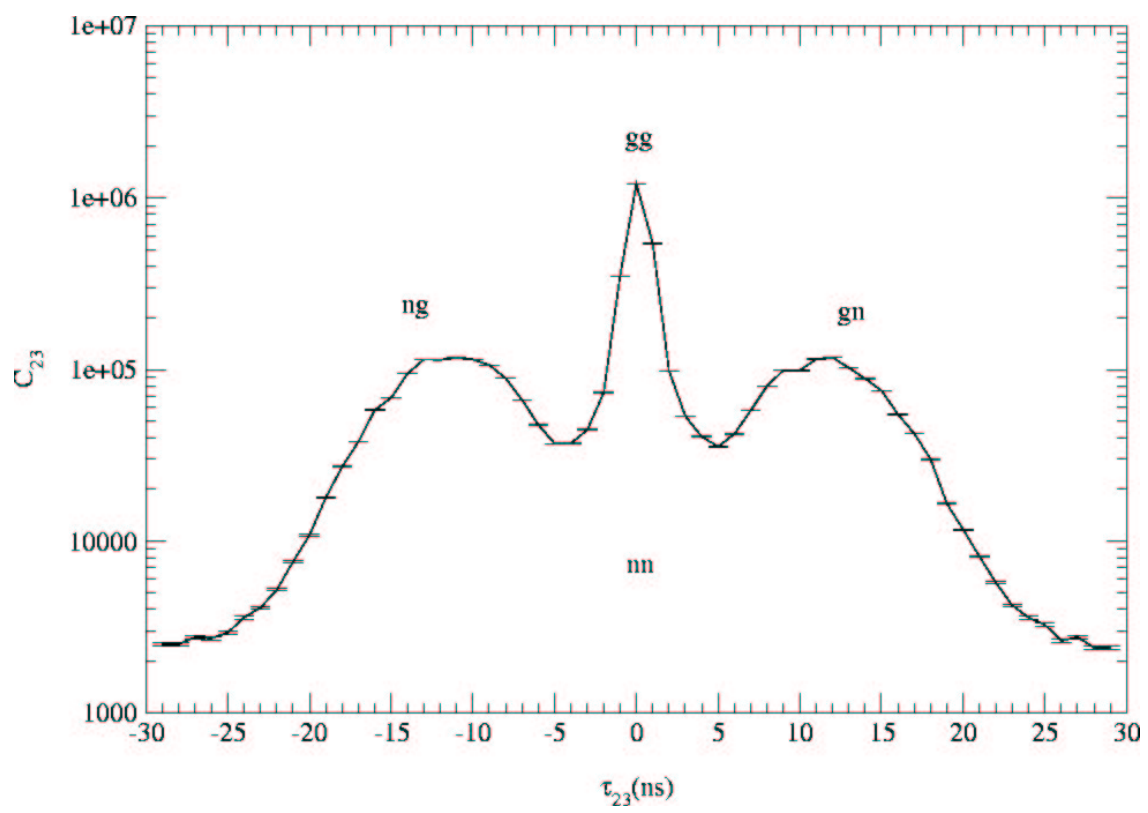

Figure 13: $C_{23}\left(\tau_{23}\right)$ in total correlated counts.

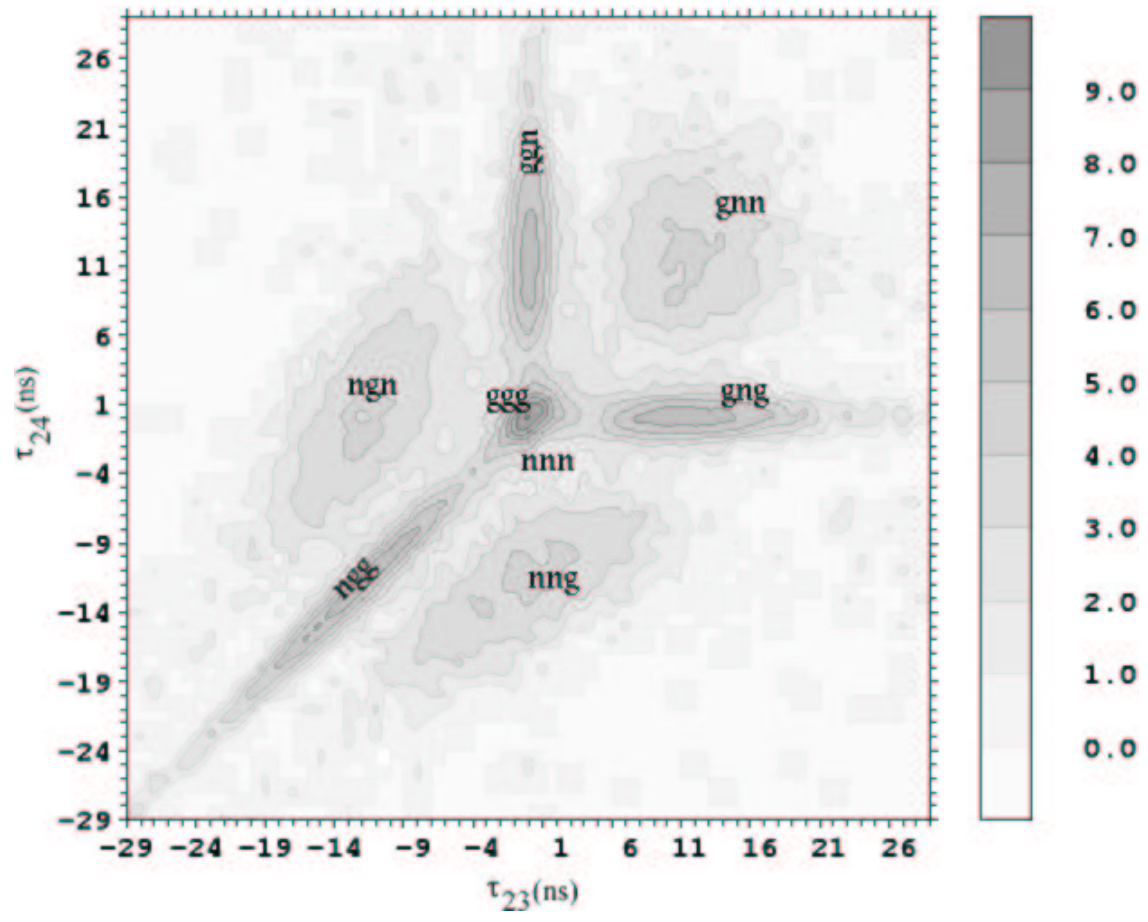

Figure 14: Measurement of $C_{234}\left(\tau_{23}, \tau_{24}\right)$ in log of total correlated counts. 
and $\left.d_{3}\right)$ is $23 \mathrm{~cm}$. An expression for this correlation can be written as

$$
\begin{aligned}
C_{234}\left(\tau_{23}, \tau_{24}\right) & =C_{g g g}\left(\tau_{23}, \tau_{24}\right) \\
& +C_{g g n}\left(\tau_{23}, \tau_{24}\right)+C_{g n g}\left(\tau_{23}, \tau_{24}\right)+C_{n g g}\left(\tau_{23}, \tau_{24}\right) \\
& +C_{g n n}\left(\tau_{23}, \tau_{24}\right)+C_{n g n}\left(\tau_{23}, \tau_{24}\right)+C_{n n g}\left(\tau_{23}, \tau_{24}\right) \\
& +C_{n n n}\left(\tau_{23}, \tau_{24}\right)
\end{aligned}
$$

where the eight terms are defined as

$$
\begin{aligned}
& C_{g g g}\left(\tau_{23}, \tau_{24}\right)=N \overline{\gamma(\gamma-1)(\gamma-2)} \epsilon_{g}^{3} \int_{0}^{\infty} \delta\left(\tau_{12}-\frac{d_{2}}{c}\right) \delta\left(\tau_{23}+\tau_{12}-\frac{d_{3}}{c}\right) \\
&\left.=N \overline{\gamma(\gamma-1)(\gamma-2)} \epsilon_{g}^{3}\right) \delta\left(\tau_{23}-\frac{d_{3}-d_{2}}{c}\right) \delta\left(\tau_{24}-\frac{d_{4}-d_{2}}{c}\right) \\
& C_{g g n}\left(\tau_{23}, \tau_{24}\right)=N \bar{\nu} \overline{\gamma(\gamma-1)} \epsilon_{g}^{2} \int_{0}^{\infty} \delta\left(\tau_{12}-\frac{d_{2}}{c}\right) \delta\left(\tau_{23}+\tau_{12}-\frac{d_{3}}{c}\right) \epsilon_{n}\left(\tau_{24}+\tau_{12}\right) \\
&\left.=N \bar{\nu} \overline{\gamma(\gamma-1)} \epsilon_{g}^{2}\right) \delta\left(\tau_{23}-\frac{d_{3}-d_{2}}{c}\right) \epsilon_{n}\left(\tau_{24}-\frac{d_{2}}{c}\right) \chi\left(\tau_{24}-\frac{d_{2}}{c}\right) \\
& C_{g n g}\left(\tau_{23}, \tau_{24}\right)=N \bar{\nu} \overline{\gamma(\gamma-1)} \epsilon_{g}^{2} \int_{0}^{\infty} \delta\left(\tau_{12}-\frac{d_{2}}{c}\right) \epsilon_{n}\left(\tau_{23}+\tau_{12}\right) \chi\left(\tau_{23}+\tau_{12}\right) \\
& C_{g n n}\left(\tau_{23}, \tau_{24}\right)= N \overline{\nu(\nu-1)} \bar{\gamma} \epsilon_{g} \int_{0}^{\infty} \delta\left(\tau_{12}-\frac{d_{2}}{c}\right) \epsilon_{n}\left(\tau_{23}+\tau_{12}\right) \chi\left(\tau_{23}+\tau_{12}\right) \epsilon_{n}\left(\tau_{24}+\tau_{12}\right) \\
&\left.=N \bar{\nu} \overline{\gamma(\gamma-1)} \epsilon_{g}^{2}\right) \epsilon_{n}\left(\tau_{23}-\frac{d_{2}}{c}\right) \chi\left(\tau_{23}-\frac{d_{2}}{c}\right) \delta\left(\tau_{24}-\frac{d_{3}-d_{2}}{c}\right) \\
& C_{n g g}\left(\tau_{23}, \tau_{24}\right)= N \bar{\nu} \overline{\gamma(\gamma-1)} \epsilon_{g}^{2} \int_{0}^{\infty} \epsilon_{n}\left(\tau_{12}\right) \chi\left(\tau_{12}\right) \delta\left(\tau_{23}+\tau_{12}-\frac{d_{3}}{c}\right) \\
& \cdot \delta\left(\tau_{24}+\tau_{12}-\frac{d_{4}}{c}\right) d \tau_{12}
\end{aligned}
$$




$$
\begin{gathered}
C_{n g n}\left(\tau_{23}, \tau_{24}\right)=N \overline{\nu(\nu-1)} \bar{\gamma} \epsilon_{g} \int_{0}^{\infty} \epsilon_{n}\left(\tau_{12}\right) \chi\left(\tau_{12}\right) \delta\left(\tau_{23}+\tau_{12}-\frac{d_{3}}{c}\right) \epsilon_{n}\left(\tau_{24}+\tau_{12}\right) \\
\cdot \chi\left(\tau_{24}+\tau_{12}\right) d \tau_{12} \\
C_{n n g}\left(\tau_{23}, \tau_{24}\right)=N \overline{\nu(\nu-1)} \bar{\gamma} \epsilon_{g} \int_{0}^{\infty} \epsilon_{n}\left(\tau_{12}\right) \chi\left(\tau_{12}\right) \epsilon_{n}\left(\tau_{23}+\tau_{12}\right) \chi\left(\tau_{23}+\tau_{12}\right) \\
\cdot \delta\left(\tau_{24}+\tau_{12}-\frac{d_{4}}{c}\right) d \tau_{12} \\
C_{n n n}\left(\tau_{23}, \tau_{24}\right)=N \frac{N \nu(\nu-1)(\nu-2)}{\nu} \int_{0}^{\infty} \epsilon_{n}\left(\tau_{12}\right) \chi\left(\tau_{12}\right) \epsilon_{n}\left(\tau_{23}+\tau_{12}\right) \chi\left(\tau_{23}+\tau_{12}\right) \\
\cdot \epsilon_{n}\left(\tau_{24}+\tau_{12}\right) \chi\left(\tau_{24}+\tau_{12}\right) d \tau_{12} .
\end{gathered}
$$

The bicorrelation as computed by Equation (46) is shown in Figure 15. The bicorrelation $C_{234}\left(\tau_{23}, \tau_{24}\right)$ has eight regions formed by all combinations of gamma and neutron detections in the three detectors. It is calculated in a manner analogous to $C_{23}\left(\tau_{23}\right)$ from the three correlations $C_{12}\left(\tau_{12}\right), C_{13}\left(\tau_{13}\right)$ and $C_{14}\left(\tau_{14}\right)$ and a substitution of the variables $\tau_{23}=\tau_{13}-\tau_{12}$ and $\tau_{24}=\tau_{14}-\tau_{12}$. The correlations have three components: the number of spontaneous fissions $N$, the factorial moments, the detection efficiency for gamma rays and neutrons, and the time distribution function. An alternative derivation of the factorial moments is provided in Appendix A.2 with the results in Equation (150).

The boundaries of the eight regions are shown in Table 4. The triplets of gamma ray detections essentially form a point with a width of $\Delta_{g}$. The boundary forms a hexagon from six of the eight combinations of $\pm \frac{\Delta_{g}}{2}$. The all minus and all plus combinations occur within the interior of this region. The triplets of neutrons are also bounded by a hexagon formed by the six combinations of fast and threshold neutrons. The two combinations with three fast and three threshold neutrons also occur within the boundary. There are three regions comprised of two gamma rays correlated with one neutron. Each of these regions form essentially a line with a width of $\Delta_{g}$. 
Table 4: Temporal bounds for $C_{234}\left(\tau_{23}, \tau_{24}\right)$

\begin{tabular}{|c|c|c|c|c|c|c|}
\hline Correlation & Combination & $\tau_{12}$ & $\tau_{13}$ & $\tau_{14}$ & $\tau_{23}$ & $\tau_{24}$ \\
\hline$C_{g g g}$ & $g g g$ & $\frac{d_{2}}{c} \pm \frac{\Delta_{g}}{2}$ & $\frac{d_{3}}{c} \pm \frac{\Delta_{g}}{2}$ & $\frac{d_{4}}{c} \pm \frac{\Delta_{g}}{2}$ & $\frac{d_{3}-d_{2}}{c} \pm \frac{\Delta_{g}}{2}$ & $\frac{d_{4}-d_{2}}{c} \pm \frac{\Delta_{g}}{2}$ \\
\hline \multirow{2}{*}{$C_{g g n}$} & $g g n_{f}$ & $\frac{d_{2}}{c} \pm \frac{\Delta_{g}}{2}$ & $\frac{d_{3}}{c} \pm \frac{\Delta_{g}}{2}$ & $\frac{d_{4}}{v_{f}}$ & $\frac{d_{3}-d_{2}}{c} \pm \frac{\Delta_{g}}{2}$ & $\frac{d_{4}}{v_{f}}-\frac{d_{2}}{c}$ \\
\hline & $g g n_{t}$ & $\frac{d_{2}}{c} \pm \frac{\Delta_{g}}{2}$ & $\frac{d_{3}}{c} \pm \frac{\Delta_{g}}{2}$ & $\frac{d_{4}}{v_{t}}$ & $\frac{d_{3}-d_{2}}{c} \pm \frac{\Delta_{g}}{2}$ & $\frac{d_{4}}{v_{t}}-\frac{d_{2}}{c}$ \\
\hline \multirow{2}{*}{$C_{g n g}$} & $g n_{f} g$ & $\frac{d_{2}}{c} \pm \frac{\Delta_{g}}{2}$ & $\frac{d_{3}}{v_{f}}$ & $\frac{d_{4}}{c} \pm \frac{\Delta_{g}}{2}$ & $\frac{d_{3}}{v_{f}}-\frac{d_{2}}{c}$ & $\frac{d_{4}-d_{2}}{c} \pm \frac{\Delta_{g}}{2}$ \\
\hline & $g n_{t} g$ & $\frac{d_{2}}{c} \pm \frac{\Delta_{g}}{2}$ & $\frac{d_{3}}{v_{t}}$ & $\frac{d_{4}}{c} \pm \frac{\Delta_{g}}{2}$ & $\frac{d_{3}}{v_{t}}-\frac{d_{2}}{c}$ & $\frac{d_{4}-d_{2}}{c} \pm \frac{\Delta_{g}}{2}$ \\
\hline \multirow{2}{*}{$C_{n g g}$} & $n_{f} g g$ & $\frac{d_{2}}{v_{f}}$ & $\frac{d_{3}}{c} \pm \frac{\Delta_{g}}{2}$ & $\frac{d_{4}}{c} \pm \frac{\Delta_{g}}{2}$ & $\frac{d_{3}}{c}-\frac{d_{2}}{v_{f}} \pm \frac{\Delta_{g}}{2}$ & $\frac{d_{4}}{c}-\frac{d_{2}}{v_{f}} \pm \frac{\Delta_{g}}{2}$ \\
\hline & $n_{t} g g$ & $\frac{d_{2}}{v_{t}}$ & $\frac{d_{3}}{c} \pm \frac{\Delta_{g}}{2}$ & $\frac{d_{4}}{c} \pm \frac{\Delta_{g}}{2}$ & $\frac{d_{3}}{c}-\frac{d_{2}}{v_{t}} \pm \frac{\Delta_{g}}{2}$ & $\frac{d_{4}}{c}-\frac{d_{2}}{v_{t}} \pm \frac{\Delta_{g}}{2}$ \\
\hline \multirow{4}{*}{$C_{g n n}$} & $g n_{f} n_{f}$ & $\frac{d_{2}}{c} \pm \frac{\Delta_{g}}{2}$ & $\frac{d_{3}}{v_{f}}$ & $\frac{d_{4}}{v_{f}}$ & $\frac{d_{3}}{v_{f}}-\frac{d_{2}}{c}$ & $\frac{d_{4}}{v_{f}}-\frac{d_{2}}{c}$ \\
\hline & $g n_{f} n_{t}$ & $\frac{d_{2}}{c} \pm \frac{\Delta_{g}}{2}$ & $\frac{d_{3}}{v_{f}}$ & $\frac{d_{4}}{v_{t}}$ & $\frac{d_{3}}{v_{f}}-\frac{d_{2}}{c}$ & $\frac{d_{4}}{v_{t}}-\frac{d_{2}}{c}$ \\
\hline & $g n_{t} n_{f}$ & $\frac{d_{2}}{c} \pm \frac{\Delta_{g}}{2}$ & $\frac{d}{v_{t}}$ & $\frac{d_{4}}{v_{f}}$ & $\frac{d_{3}}{v_{t}}-\frac{d_{2}}{c}$ & $\frac{d_{4}}{v_{f}}-\frac{d_{2}}{c}$ \\
\hline & $g n_{t} n_{t}$ & $\frac{d_{2}}{c} \pm \frac{\Delta_{g}}{2}$ & $\frac{d_{3}}{v_{t}}$ & $\frac{d_{4}}{v_{t}}$ & $\frac{d_{3}}{v_{t}}-\frac{d_{2}}{c}$ & $\frac{d_{4}}{v_{t}}-\frac{d_{2}}{c}$ \\
\hline \multirow{4}{*}{$C_{n g n}$} & $n_{f} g n_{f}$ & $\frac{d_{2}}{v_{f}}$ & $\frac{d_{3}}{c} \pm \frac{\Delta_{g}}{2}$ & $\frac{d_{4}}{v_{f}}$ & $\frac{d_{3}}{c}-\frac{d_{2}}{v_{f}}$ & $\frac{d_{4}}{v_{f}}-\frac{d_{2}}{v_{f}}$ \\
\hline & $n_{f} g n_{t}$ & $\frac{d_{2}}{v_{f}}$ & $\frac{d_{3}}{c} \pm \frac{\Delta_{g}}{2}$ & $\frac{d_{4}}{v_{t}}$ & $\frac{d_{3}}{c}-\frac{d_{2}}{v_{f}}$ & $\frac{d_{4}}{v_{t}}-\frac{d_{2}}{v_{f}}$ \\
\hline & $n_{t} g n_{f}$ & $\frac{d_{2}}{v_{t}}$ & $\frac{d_{3}}{c} \pm \frac{\Delta_{g}}{2}$ & $\frac{d_{4}}{v_{f}}$ & $\frac{d_{3}}{c}-\frac{d_{2}}{v_{t}}$ & $\frac{d_{4}}{v_{f}}-\frac{d_{2}}{v_{t}}$ \\
\hline & $n_{t} g n_{t}$ & $\frac{d_{2}}{v_{t}}$ & $\frac{d_{3}}{c} \pm \frac{\Delta_{g}}{2}$ & $\frac{d_{4}}{v_{t}}$ & $\frac{d_{3}}{c}-\frac{d_{2}}{v_{t}}$ & $\frac{d_{4}}{v_{t}}-\frac{d_{2}}{v_{t}}$ \\
\hline \multirow{4}{*}{$C_{n n g}$} & $n_{f} n_{f} g$ & $\frac{d_{2}}{v_{f}}$ & $\frac{d_{3}}{v_{f}}$ & $\frac{d_{4}}{c} \pm \frac{\Delta_{g}}{2}$ & $\frac{d_{3}}{v_{f}}-\frac{d_{2}}{v_{f}}$ & $\frac{d_{4}}{c}-\frac{d_{2}}{v_{f}}$ \\
\hline & $n_{f} n_{t} g$ & $\frac{d_{2}}{v_{f}}$ & $\frac{d_{3}}{v_{t}}$ & $\frac{d_{4}}{c} \pm \frac{\Delta_{g}}{2}$ & $\frac{d_{3}}{v_{t}}-\frac{d_{2}}{v_{f}}$ & $\frac{d_{4}}{c}-\frac{d_{2}}{v_{f}}$ \\
\hline & $n_{t} n_{f} g$ & $\frac{d_{2}}{v_{t}}$ & $\frac{d_{3}}{v_{f}}$ & $\frac{d_{4}}{c} \pm \frac{\Delta_{g}}{2}$ & $\frac{d_{3}}{v_{f}}-\frac{d_{2}}{v_{t}}$ & $\frac{d_{4}}{c}-\frac{d_{2}}{v_{t}}$ \\
\hline & $n_{t} n_{t} g$ & $\frac{d_{2}}{v_{t}}$ & $\frac{d_{3}}{v_{t}}$ & $\frac{d_{4}}{c} \pm \frac{\Delta_{g}}{2}$ & $\frac{d_{3}}{v_{t}}-\frac{d_{2}}{v_{t}}$ & $\frac{d_{4}}{c}-\frac{d_{2}}{v_{t}}$ \\
\hline \multirow{8}{*}{$C_{n n n}$} & $n_{f} n_{f} n_{f}$ & $\frac{d_{2}}{v_{f}}$ & $\frac{d_{3}}{v_{f}}$ & $\frac{d_{4}}{v_{f}}$ & - & - \\
\hline & $n_{f} n_{f} n_{t}$ & $\frac{d_{2}}{v_{f}}$ & $\frac{d_{3}}{v_{f}}$ & $\frac{d_{4}}{v_{t}}$ & $\frac{d_{3}}{v_{f}}-\frac{d_{2}}{v_{f}}$ & $\frac{d_{4}}{v_{t}}-\frac{d_{2}}{v_{f}}$ \\
\hline & $n_{f} n_{t} n_{f}$ & $\frac{d_{2}}{v_{f}}$ & $\frac{d_{3}}{v_{t}}$ & $\frac{d_{4}}{v_{f}}$ & $\frac{d_{3}}{v_{t}}-\frac{d_{2}}{v_{f}}$ & $\frac{d_{4}}{v_{f}}-\frac{d_{2}}{v_{f}}$ \\
\hline & $n_{f} n_{t} n_{t}$ & $\frac{d_{2}}{v_{f}}$ & $\frac{d_{3}}{v_{t}}$ & $\frac{d_{4}}{v_{t}}$ & $\frac{d_{3}}{v_{t}}-\frac{d_{2}}{v_{f}}$ & $\frac{d_{4}}{v_{t}}-\frac{d_{2}}{v_{f}}$ \\
\hline & $n_{t} n_{f} n_{f}$ & $\frac{d_{2}}{v_{t}}$ & $\frac{d_{3}}{v_{f}}$ & $\frac{d_{4}}{v_{f}}$ & $\frac{d_{3}}{v_{f}}-\frac{d_{2}}{v_{t}}$ & $\frac{d_{4}}{v_{f}}-\frac{d_{2}}{v_{t}}$ \\
\hline & $n_{t} n_{f} n_{t}$ & $\frac{d_{2}}{v_{t}}$ & $\frac{d_{3}}{v_{f}}$ & $\frac{d_{4}}{v_{t}}$ & $\frac{d_{3}}{v_{f}}-\frac{d_{2}}{v_{t}}$ & $\frac{d_{4}}{v_{t}}-\frac{d_{2}}{v_{t}}$ \\
\hline & $n_{t} n_{t} n_{f}$ & $\frac{d_{2}}{v_{t}}$ & $\frac{d_{3}}{v_{t}}$ & $\frac{d_{4}}{v_{f}}$ & $\frac{d_{3}}{v_{t}}-\frac{d_{2}}{v_{t}}$ & $\frac{d_{4}}{v_{f}}-\frac{d_{2}}{v_{t}}$ \\
\hline & $n_{t} n_{t} n_{t}$ & $\frac{d_{2}}{v_{t}}$ & $\frac{d_{3}}{v_{t}}$ & $\frac{d_{4}}{v_{t}}$ & . & \\
\hline
\end{tabular}




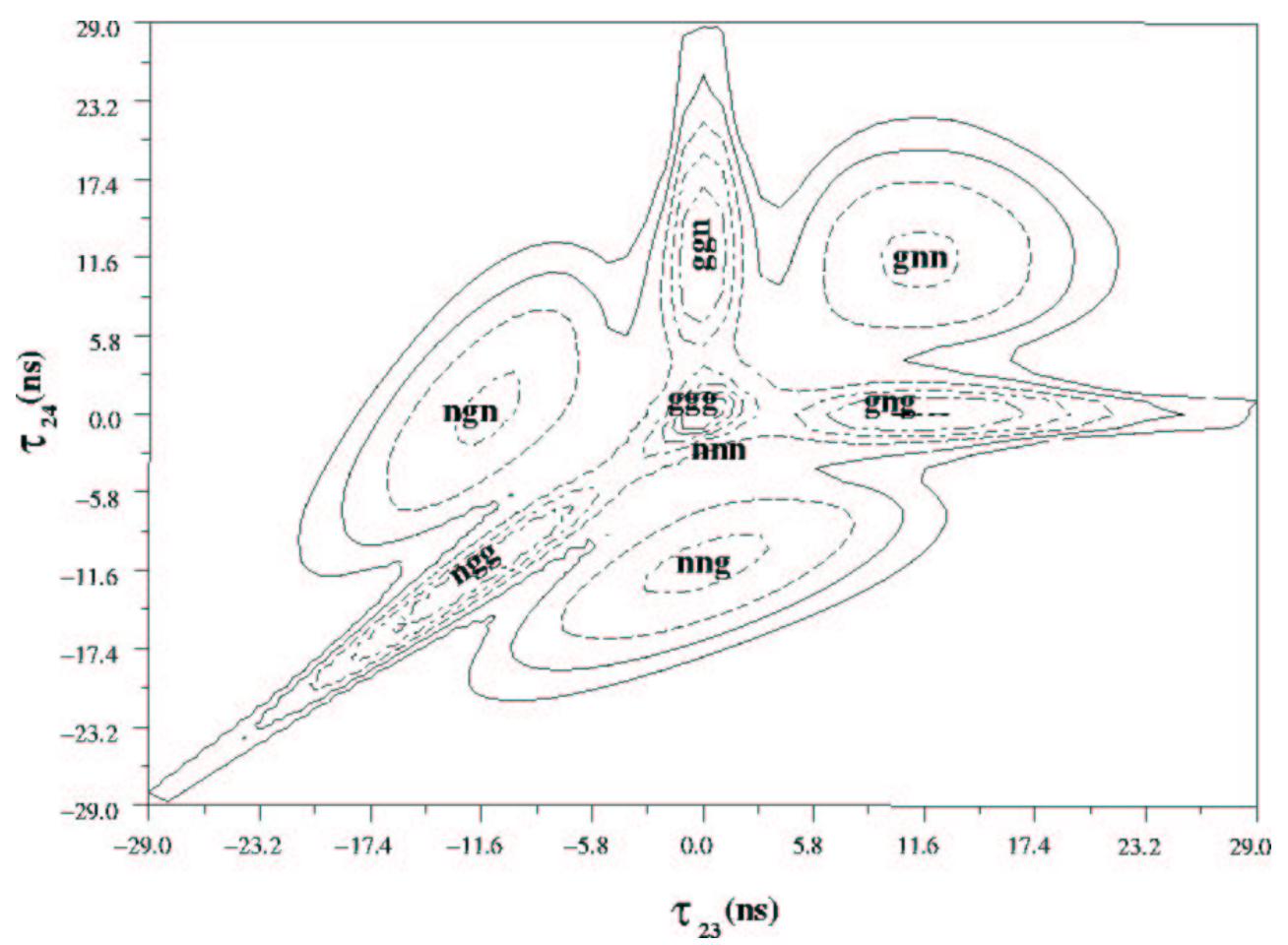

Figure 15: $C_{234}\left(\tau_{23}, \tau_{24}\right)$ calculated from Equation (46).

\section{$\underline{4.2}$ Ratios of source-detector correlations}

Active measurements (source correlated) provide several different ratios between pairs and triplets of detections. The first which was previously considered is the ratio of the detector cross-correlation with the source correlated detector cross-correlation.

$$
\frac{C_{23 \mid 1}\left(\tau_{23}\right)}{C_{23}\left(\tau_{23}\right)}=\frac{N_{1} \epsilon_{f}}{N_{1}+N_{b}}
$$

In this equation $N_{b}$ is a background source which produces correlated detections in detectors 2 and $3 .^{3} \quad$ The background could be a correlated gamma source such as fission products or ${ }^{238} U$ alpha decay products. Even an uncorrelated source can produce correlated detections through detector-detector scattering.[43]

Equation (55) will be examined in more detail here with an example. Assume for the moment the the background is comprised of gamma ray coincidences produced by beta

\footnotetext{
${ }^{3}$ Mattingly makes use of this relation to remove background from the detector-detector correlations. [32]
} 
decay of fission products. The ratio can be written

$$
\frac{C_{23 \mid 1}\left(\tau_{23}\right)}{C_{23}\left(\tau_{23}\right)}=\frac{C_{g g \mid f_{1}}\left(\tau_{23}\right)+C_{g n \mid f_{1}}\left(\tau_{23}\right)+C_{n g \mid f_{1}}\left(\tau_{23}\right)+C_{n n \mid f_{1}}\left(\tau_{23}\right)}{C_{g g}\left(\tau_{23}\right)+C_{g n}\left(\tau_{23}\right)+C_{n g}\left(\tau_{23}\right)+C_{n n}\left(\tau_{23}\right)+C_{r g g}\left(\tau_{23}\right)} .
$$

With a source-to-detector distance of $23 \mathrm{~cm}$ for both detectors, the neutron pairs extend from \pm 12 ns. Beyond this range the $n n$ correlation approaches zero. The $n g$ pairs extend to $-25 \mathrm{~ns}$ and the $g n$ pairs extend to $25 \mathrm{~ns}$. Gamma pairs occur between \pm 5 ns. From $-25 \mathrm{~ns}$ to $-12 \mathrm{~ns}$, all of the terms are zero except $C_{n g \mid f_{1}}\left(\tau_{23}\right)$ and $C_{n g}\left(\tau_{23}\right)$. The ratio becomes

$$
\begin{aligned}
\frac{C_{23 \mid 1}\left(\tau_{23}\right)}{C_{23}\left(\tau_{23}\right)} & =\frac{C_{n g \mid f_{1}}\left(\tau_{23}\right)}{C_{n g}\left(\tau_{23}\right)} \\
& =\frac{N_{1} \epsilon_{f} \overline{\nu \gamma} \epsilon_{g} \int_{0}^{\infty} \epsilon_{n}\left(\tau_{12}\right) \chi\left(\tau_{12}\right) \delta\left(\tau_{23}+\tau_{12}-\frac{d_{3}}{c}\right) d \tau_{12}}{N_{1} \overline{\nu \gamma} \epsilon_{g} \int_{0}^{\infty} \epsilon_{n}\left(\tau_{12}\right) \chi\left(\tau_{12}\right) \delta\left(\tau_{23}+\tau_{12}-\frac{d_{3}}{c}\right) d \tau_{12}} \\
& =\frac{N_{1} \epsilon_{f}}{N_{1}}=\epsilon_{f} .
\end{aligned}
$$

The region from $12 \mathrm{~ns}$ to $25 \mathrm{~ns}$ on the $\tau_{23}$ axis is identical. The region at zero $\pm 5 \mathrm{~ns}$ is dominated by gamma pairs. Although there are some $n n$ pairs in this region they are lower by several orders of magnitude. The ratio in this region then becomes

$$
\begin{aligned}
\frac{C_{23 \mid 1}\left(\tau_{23}\right)}{C_{23}\left(\tau_{23}\right)} & =\frac{C_{g g \mid f_{1}}\left(\tau_{23}\right)}{C_{g g}\left(\tau_{23}\right)+C_{r g g}\left(\tau_{23}\right.} \\
& =\frac{N_{1} \epsilon_{f} \overline{\gamma(\gamma-1)} \epsilon_{g}^{2} \delta\left(\tau_{23}-\frac{d_{3}-d_{2}}{c}\right)}{N_{1} \overline{\gamma(\gamma-1)} \epsilon_{g}^{2} \delta\left(\tau_{23}-\frac{d_{3}-d_{2}}{c}\right)+N_{r} \overline{\gamma_{r}\left(\gamma_{r}-1\right)} \epsilon_{g}^{2} \delta\left(\tau_{23}-\frac{d_{3}-d_{2}}{c}\right)} \\
& =\frac{N_{1} \epsilon_{f} \overline{\gamma(\gamma-1)}}{N_{1} \overline{\gamma(\gamma-1)}+N_{r} \overline{\gamma_{r}\left(\gamma_{r}-1\right)}}
\end{aligned}
$$

where $N_{r}$ is the number of radioactive decays from the fission products. In the last step, an assumption was made that the efficiency for detecting gamma rays from fission was the same as the efficiency for decay gamma rays. This is of course not necessarily so because of the energy differences. The results of this ratio are shown in Figure 42 in Section 8.1.

A second ratio is the source-detector outer product with the $C_{123}$ correlation,

$$
\frac{C_{12}\left(\tau_{12}\right) C_{13}\left(\tau_{13}\right)}{N_{1} \epsilon_{f} C_{123}\left(\tau_{12}, \tau_{13}\right)}=\underbrace{\frac{\bar{\gamma}^{2}}{\overline{\gamma(\gamma-1)}}}_{\mathrm{gg}}+\underbrace{\frac{\bar{\nu}^{2}}{\overline{\nu(\nu-1)}}}_{\mathrm{nn}}+\underbrace{1}_{\mathrm{ng} \text { or gn }}
$$


where each term occupies its own region in the $\tau_{12}, \tau_{13}$ plane. This ratio has the advantage that both numerator and denominator are source correlated. The background term $N_{b}$ is not present.

The numerator and denominator of this ratio can also be integrated into $\tau_{23}=\tau_{13}-\tau_{12}$ as follows:

$$
\frac{\int_{0}^{\infty} C_{12}\left(\tau_{12}\right) C_{13}\left(\tau_{23}+\tau_{12}\right) d \tau_{12}}{N_{1} \epsilon_{f} C_{23 \mid 1}\left(\tau_{23}\right)}=\underbrace{\frac{\bar{\gamma}^{2}}{\overline{\gamma(\gamma-1)}}}_{\mathrm{gg}}+\underbrace{\frac{\bar{\nu}^{2}}{\overline{\nu(\nu-1)}}}_{\mathrm{nn}}+\underbrace{1}_{\text {ng or gn }} .
$$

A similar ratio can be formed from the product of all three source-to-detector correlations: $C_{12}\left(\tau_{12}\right), C_{13}\left(\tau_{13}\right)$ and $C_{14}\left(\tau_{14}\right)$. This time the ratio is a function of the two variables $\tau_{23}=\tau_{13}-\tau_{12}$ and $\tau_{24}=\tau_{14}-\tau_{12}$.

$$
\begin{aligned}
& \frac{\int_{0}^{\infty} C_{12}\left(\tau_{12}\right) C_{13}\left(\tau_{23}+\tau_{12}\right) C_{14}\left(\tau_{24}+\tau_{12}\right) d \tau_{12}}{\left(N_{1} \epsilon_{f}\right)^{2} C_{234}\left(\tau_{23}, \tau_{24}\right)}=\underbrace{\frac{\bar{\gamma}^{3}}{\overline{\gamma(\gamma-1)(\gamma-2)}}}_{\text {ggg }}+\underbrace{\frac{\bar{\nu}^{3}}{\overline{\nu(\nu-1)(\nu-2)}}}_{\mathrm{nnn}}
\end{aligned}
$$

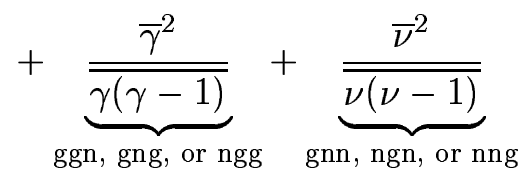

The autocorrelation of the source is also related to source fission rate and fission detection efficiency. The relation is

$$
C_{11}(0)=N_{1} \epsilon_{f}+N_{\alpha}
$$

where $N_{\alpha}$ is the number of false fission detections.

\subsection{Ratios of detector-detector correlations in passive measurement}

Various ratios of the form $\frac{C_{i j}\left(\tau_{i j}\right)}{C_{i j \mid k}\left(\tau_{i j}\right)}$ are possible. For three detectors $(2,3$ and 4$)$ there are three conditional correlations contained in the bicorrelation $C_{234}\left(\tau_{23}, \tau_{24}\right)$. Using the 
relation $\tau_{34}=\tau_{23}-\tau_{24}$, these three conditional correlations are

$$
\begin{aligned}
C_{23 \mid 4}\left(\tau_{23}\right) & =\int_{0}^{\infty} C_{234}\left(\tau_{23}, \tau_{24}\right) d \tau_{24} \\
C_{24 \mid 3}\left(\tau_{24}\right) & =\int_{0}^{\infty} C_{234}\left(\tau_{23}, \tau_{24}\right) d \tau_{23} \\
C_{34 \mid 2}\left(\tau_{34}\right) & =\int_{0}^{\infty} C_{234}\left(\tau_{34}+\tau_{24}, \tau_{24}\right) d \tau_{24} \\
& =\int_{0}^{\infty} C_{234}\left(\tau_{23}, \tau_{23}-\tau_{34}\right) d \tau_{23} .
\end{aligned}
$$

The time dependence can be eliminated with a second integration. The equations then become

$$
\begin{aligned}
C_{23 \mid 4} & =\int_{0}^{\infty} \int_{0}^{\infty} C_{234}\left(\tau_{23}, \tau_{24}\right) d \tau_{23} d \tau_{24} \\
C_{24 \mid 3} & =\int_{0}^{\infty} \int_{0}^{\infty} C_{234}\left(\tau_{23}, \tau_{24}\right) d \tau_{23} d \tau_{24} \\
C_{34 \mid 2} & =\int_{0}^{\infty} \int_{0}^{\infty} C_{234}\left(\tau_{34}+\tau_{24}, \tau_{24}\right) d \tau_{24} d \tau_{34} \\
& =\int_{0}^{\infty} \int_{0}^{\infty} C_{234}\left(\tau_{23}, \tau_{23}-\tau_{34}\right) d \tau_{23} d \tau_{34} .
\end{aligned}
$$

In addition, the correlations can be separated by reaction type. For example $C_{i j \mid k}(g g \mid n)$ represents a gamma detection in both detector $i$ and $j$ given that a neutron was detected in detector $k$. This conditional correlation is a function of $\tau_{i j}$ and comes from doing the appropriate integration on $C_{i j k}$. It can also conveniently be written as $C_{g g \mid n}\left(\tau_{i j}\right)$. Some of these ratios are

$$
\begin{gathered}
\frac{C_{g g \mid n}}{C_{g g}}=\bar{\nu} \epsilon_{n}, \\
\frac{C_{g n \mid g}\left(\tau_{i j}\right)}{C_{g n}\left(\tau_{i j}\right)}=\frac{\overline{\gamma(\gamma-1)}}{\bar{\gamma}} \epsilon_{g}, \\
\frac{C_{g n \mid n}\left(\tau_{i j}\right)}{C_{g n}\left(\tau_{i j}\right)}=\frac{\overline{\nu(\nu-1)}}{\bar{\nu}} \epsilon_{n}\left(\tau_{i j}\right),
\end{gathered}
$$




$$
\begin{gathered}
\frac{\left(C_{g n}\left(\tau_{i j}\right)\right)^{2}}{C_{g n \mid g}\left(\tau_{i j}\right)}=N \frac{\bar{\gamma}^{2} \bar{\nu}}{\overline{\gamma(\gamma-1)}} \epsilon_{n}\left(\tau_{i j}\right), \\
\frac{\left(C_{g n}\left(\tau_{i j}\right)\right)^{2}}{C_{g n \mid n}\left(\tau_{i j}\right)}=N \frac{\bar{\nu}^{2} \bar{\gamma}}{\overline{\nu(\nu-1)}} \epsilon_{g}, \\
\frac{C_{g g} C_{g n}}{C_{g n \mid g}}=N \bar{\gamma} \epsilon_{g}, \\
\frac{C_{n n}\left(\tau_{i j}\right) C_{g n}\left(\tau_{i j}\right)}{C_{g n \mid n}\left(\tau_{i j}\right)}=N \bar{\nu} \epsilon_{n}\left(\tau_{i j}\right), \\
\frac{C_{g g}}{C_{g g g}}=\frac{\frac{\gamma(\gamma-1)}{\gamma(\gamma-1)(\gamma-2)} \epsilon_{g}}{\overline{\gamma(\gamma-1}}
\end{gathered}
$$

Other ratios are of course conceivable. For example, the ratios involving pairs and triplets of only neutrons were omitted.

\subsection{Multiplicity data}

The factorial moments of the neutron and gamma ray multiplicities are calculated from data from various sources. A description of this data is given in the following two sections.

\subsubsection{Neutron multiplicity}

Table 5 lists the probabilities for the neutron multiplicities for the spontaneous fissioning isotopes, ${ }^{252} \mathrm{Cf}$ and ${ }^{240} \mathrm{Pu}$.[48] The first two factorial moments are also shown.

\subsubsection{Gamma-ray multiplicity}

The gamma ray multiplicity distribution from fission, $\gamma(G)$ can be computed using varying models.[49] One model is the double Poisson model used by Brunson[8] where $\gamma$ represents the number of prompt fission gamma rays.

$$
p(\gamma)=C_{1} \frac{C_{2}^{\gamma} e^{-C_{2}}}{\gamma !}+\left(1-C_{1}\right) \frac{C_{3}^{\gamma} e^{-C_{3}}}{\gamma !}
$$


Table 5: Neutron multiplicities and factorial moments

\begin{tabular}{|c|c|c|}
\cline { 2 - 3 } \multicolumn{1}{c|}{} & ${ }^{252} C f$ & $\mathrm{Pu}$ \\
\hline$\nu$ & $p_{\nu}$ & $p_{\nu}$ \\
\hline 0 & 0.00211 & 0.0631852 \\
1 & 0.02467 & 0.2319644 \\
2 & 0.12290 & 0.3333230 \\
3 & 0.27144 & 0.2528207 \\
4 & 0.30763 & 0.0986461 \\
5 & 0.18770 & 0.0180199 \\
6 & 0.06770 & 0.0020406 \\
7 & 0.01406 & 0.0 \\
8 & 0.00167 & 0.0 \\
9 & 0.00010 & 0.0 \\
\hline $\bar{\nu}$ & 3.77269 & 2.154 \\
$\overline{\nu(\nu-1)}$ & 12.04224 & 3.78894 \\
$\overline{\nu(\nu-1)(\nu-2)}$ & 31.96188 & 5.21050 \\
\hline
\end{tabular}

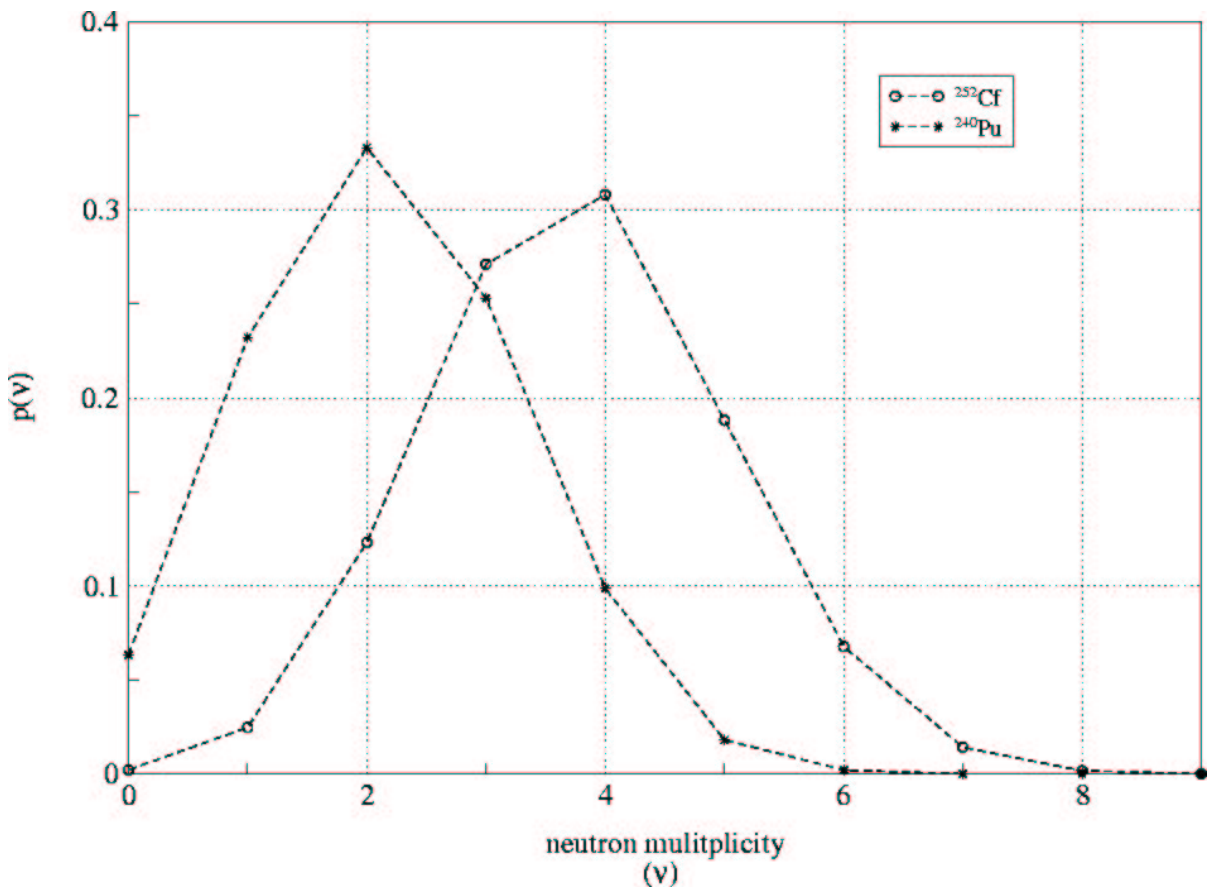

Figure 16: Neutron multiplicities for ${ }^{252} \mathrm{Cf}$ and ${ }^{240} \mathrm{Pu}$. 
The major drawback with this model is that three constants, $C_{1}, C_{2}$, and $C_{3}$ must be derived. A model that only requires two parameters is the negative binomial distribution[49]. This distribution is

$$
p(\gamma)=\left(\begin{array}{c}
\alpha^{\prime}+\gamma-1 \\
\gamma
\end{array}\right) p^{\alpha^{\prime}}(1-p)^{\gamma}
$$

where $\alpha^{\prime}$ is computed from the relative width $D_{\gamma}$ as shown in Equation (79) and $p$ is computed from $\alpha^{\prime}$ and $\bar{G}$ as shown in Equation (80).

$$
\begin{gathered}
\alpha^{\prime}=\frac{1}{D_{\gamma}-1} \\
p=\frac{\alpha^{\prime}}{\alpha^{\prime}+\bar{\gamma}}
\end{gathered}
$$

From Equation (78) the following prompt fission gamma ray multiplicities were calculated for ${ }^{252} \mathrm{Cf}$ and ${ }^{240} \mathrm{Pu}$ as shown in Table 6 and is plotted in Figure 17.

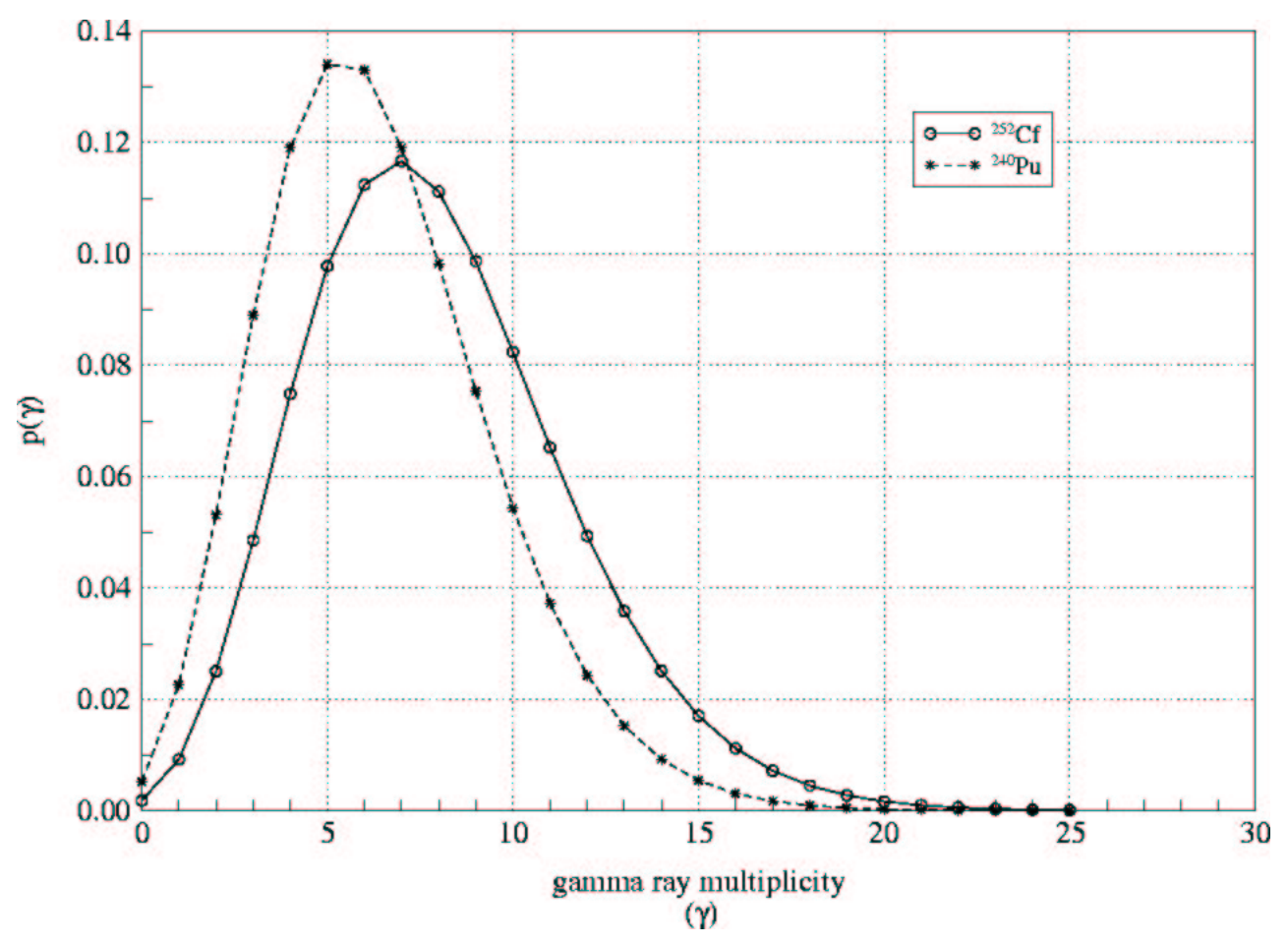

Figure 17: Gamma ray multiplicities for ${ }^{252} C f$ and ${ }^{240} \mathrm{Pu}$. 
Table 6: Gamma-ray multiplicities and factorial moments

\begin{tabular}{|c|c|c|}
\cline { 2 - 3 } \multicolumn{1}{c|}{} & ${ }^{252} C f$ & ${ }^{240} P u$ \\
\hline$\gamma$ & $p(\gamma)$ & $p(\gamma)$ \\
\hline 0 & $1.81 \mathrm{e}-03$ & $5.14 \mathrm{e}-03$ \\
1 & $9.19 \mathrm{e}-03$ & $2.26 \mathrm{e}-02$ \\
2 & $2.50 \mathrm{e}-02$ & $5.31 \mathrm{e}-02$ \\
3 & $4.85 \mathrm{e}-02$ & $8.89 \mathrm{e}-02$ \\
4 & $7.48 \mathrm{e}-02$ & $1.19 \mathrm{e}-01$ \\
5 & $9.78 \mathrm{e}-02$ & $1.34 \mathrm{e}-01$ \\
6 & $1.12 \mathrm{e}-01$ & $1.33 \mathrm{e}-01$ \\
7 & $1.17 \mathrm{e}-01$ & $1.19 \mathrm{e}-01$ \\
8 & $1.11 \mathrm{e}-01$ & $9.82 \mathrm{e}-02$ \\
9 & $9.86 \mathrm{e}-02$ & $7.53 \mathrm{e}-02$ \\
10 & $8.23 \mathrm{e}-02$ & $5.43 \mathrm{e}-02$ \\
11 & $6.52 \mathrm{e}-02$ & $3.72 \mathrm{e}-02$ \\
12 & $4.93 \mathrm{e}-02$ & $2.43 \mathrm{e}-02$ \\
13 & $3.58 \mathrm{e}-02$ & $1.52 \mathrm{e}-02$ \\
14 & $2.51 \mathrm{e}-02$ & $9.22 \mathrm{e}-03$ \\
15 & $1.70 \mathrm{e}-02$ & $5.40 \mathrm{e}-03$ \\
16 & $1.12 \mathrm{e}-02$ & $3.07 \mathrm{e}-03$ \\
17 & $7.17 \mathrm{e}-03$ & $1.70 \mathrm{e}-03$ \\
18 & $4.48 \mathrm{e}-03$ & $9.19 \mathrm{e}-04$ \\
19 & $2.74 \mathrm{e}-03$ & $4.86 \mathrm{e}-04$ \\
20 & $1.64 \mathrm{e}-03$ & $2.51 \mathrm{e}-04$ \\
21 & $9.65 \mathrm{e}-04$ & $1.28 \mathrm{e}-04$ \\
22 & $5.57 \mathrm{e}-04$ & $6.37 \mathrm{e}-05$ \\
23 & $3.17 \mathrm{e}-04$ & $3.13 \mathrm{e}-05$ \\
24 & $1.77 \mathrm{e}-04$ & $1.51 \mathrm{e}-05$ \\
25 & $9.78 \mathrm{e}-05$ & $7.22 \mathrm{e}-06$ \\
\hline $\bar{\gamma}$ & 7.98 & 6.40 \\
$\gamma(\gamma-1)$ & 68.15 & 43.88 \\
$\gamma(\gamma-1)(\gamma-2)$ & 620.18 & 320.81 \\
\hline & & \\
\hline & & \\
\hline 14 & & \\
\hline 1 & &
\end{tabular}




\subsection{Factors affecting time distribution of correlations}

For a point fission source with no intervening material between the source and detector, there is a direct relationship between neutron energy and the delay variable $\tau_{1 j}$. This relation was set out in Equation (25). The experiment described in Chapter 7 also roughly satisfies this condition, and the temporal bounds summarized in Tables 3 and 4 assume this condition. Generally in an NDA measurement, this condition will not hold. The material will be spatially distributed. The material may be in a matrix and also in a container. These conditions will affect the time correlations. In addition spontaneous fissions in one isotope may induce fissions in another isotope. This multiplication process will also effect the time correlations as well as the multiplicity moments. Each of these factors will be considered in more detail below.

The effect of these factors on the time distribution can be easily incorporated in the equations for the correlations previously described in this chapter. One merely needs to reinterpret $\epsilon_{n}(\tau)$ to include the effects of spatial distribution of the spontaneous fissioning material, scattering and multiplication. In addition, the temporal bounds summarized in Tables 3 and 4 can also be adjusted to take into account these additional factors. The effect will be more overlap primarily between the $n n$ pairs and the $n g$ regions; and $n n n$ triplets and both ggn and gnn regions.

\subsubsection{Spatial distribution of material}

Gamma-neutron temporal separation is not limited to a point source. As was previously shown, the detector correlations depend on the spatial distribution of the fissile material.[9] Although almost too trivial to state, the temporal boundaries in Tables 3 and 4 need a simple adjustment. The maximum distance $d_{i}$ from detector $i$ to any part of the sample should be used with respect to the threshold neutrons $v_{t}$. Conversely, the minimum distance should be used with respect to the fast neutrons, $v_{f}$. The parameter $\Delta_{g}$ should be made no smaller than $\frac{l}{2 c}$ where $l$ is the largest chord length through the sample. 


\subsubsection{Scattering in matrix and surrounding materials}

Scattering in matrix and adjacent materials will broaden both $\delta(\tau)$ and $\epsilon_{n}(\tau) \chi(\tau)$ distributions. This broadening will generally be toward longer delays. The scattered gamma rays will continue at the speed $c$. Scattering merely increases their effective path length to the detector. For neutrons, scattering not only increases the path length, but also always reduces the speed of the exiting neutron. There is however a limitation on the speed reduction because of the detector threshold. There is an exception to the increase in delay from scattering. The gamma from an inelastic scattered neutron can arrive before the original neutron would have. In any event the effect of scattering as well as spatial distribution of the sample material can be observed directly from the correlations.

In addition, scattering materials, which are not in the direct line of sight between the radiation source and the detector, will increase the number of radiations detected through scattering into the detector. Conversely, scattering material located directly in the line of sight between the radiation source and the detector will scatter radiations away from the detector preventing detection. Consequently, elastic scattering has a significant effect on both the magnitude and shape of both source-detector as well as detector-detector correlations.

\subsubsection{Neutron multiplication}

The multiplication process also broadens both $\delta(\tau)$ and $\epsilon_{n}(\tau) \chi(\tau)$ distributions. This effect is of course intertwined with spatial distribution and scattering. The probability of induced fission depend on the spatial distribution of the fissile material. In addition the probability of an induced fission increases as the energy of the neutrons are reduced through scattering. The effect on the time distribution of detections from the neutron multiplication process will be considered again in Section 5.1 in the chapter on multiplication.

\subsection{Correlated detections from non-fission reactions}

A number of background interactions can produce correlated detections. Beta decay, which is common among fission products, will produce correlated gamma detections. Neutron 
capture is frequently followed by a cascade of one or more gamma rays. These gamma rays will produce correlated gamma detections. In addition, neutron scattering interactions can produce correlations between gamma rays or between gamma rays and neutrons. Also common in the assay of nuclear materials are $(\alpha, n)$ reactions which produce a neutron and a cascade of up to several gamma rays. These reactions will also produce gamma ray coincidences as well as gamma-neutron coincidences between detectors. These reactions will be considered in some detail.

Both inelastic scattering and alpha-n $(\alpha, n)$ reactions will cause gamma-neutron correlations. This correlation makes both gamma-neutron correlations as well as g-g-n bicorrelations a suspect signature of fission. Both of these reactions will be discussed in more detail in the following subsections.

The multiplicity moments of these correlations are summarized below in Table 7 .

\subsubsection{Nuclear decay}

Fission products, some alpha decay products, and the products of neutron capture are typically radioactive. This radioactive decay is typically accompanied by a number of gamma rays. These gamma rays will produce $g g$ as well as $g g g$ coincidences. The equation for the $g g$ correlation is virtually identical to that for the $C_{g g}$ component for fission. It can be written as

$$
C_{g g \mid r}\left(\tau_{23}\right)=N_{r} \overline{\gamma_{r}\left(\gamma_{r}-1\right)} \epsilon_{g}^{2} \delta\left(\tau_{23}-\frac{d_{3}-d_{2}}{c}\right)
$$

where $N_{r}$ is the number of such decays and $\gamma_{r}$ is the multiplicity for the decay. The correlation for gamma triplets for nuclear decay is

$$
C_{g g g \mid r}\left(\tau_{23}, \tau_{24}\right)=N_{r} \overline{\gamma_{r}\left(\gamma_{r}-1\right)\left(\gamma_{r}-2\right)} \epsilon_{g}^{3} \delta\left(\tau_{23}-\frac{d_{3}-d_{2}}{c}\right) \delta\left(\tau_{24}-\frac{d_{4}-d_{2}}{c}\right) .
$$

The gamma detection efficiency $\epsilon_{g}$ will probably be different for gamma rays from radioactive decay than for gamma rays from fission. 


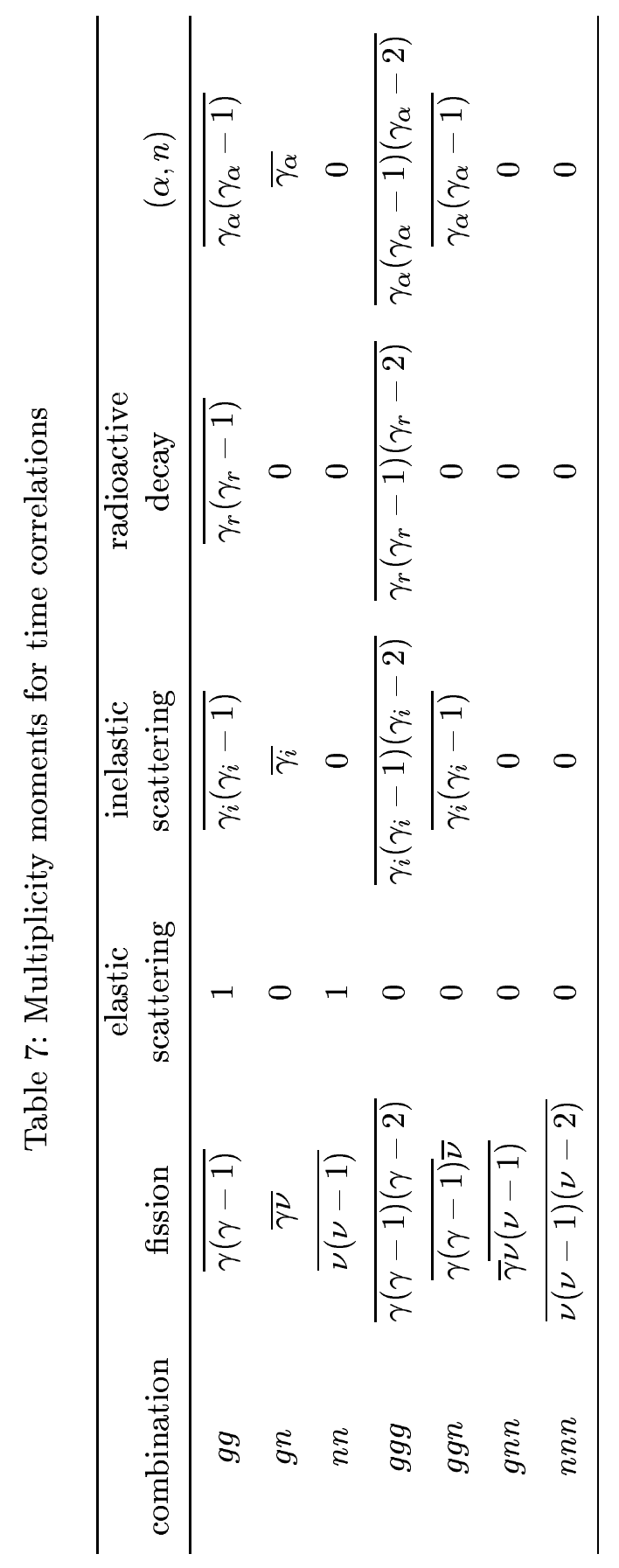




\subsubsection{Elastic scattering}

Elastic scattering can produce detector-detector correlations through cross-talk $[43,15,18$, $26,53,42]$ between the detectors. This arises when a neutron scatters from one detector to another while depositing enough energy in each for a detection. All elastic scattering delays the detection of the neutron. The distance the neutron travels from its birth to detection will always be increased by elastic scatter. In addition the velocity of the neutron will be decreased after the scatter. This effect will fortunately not cause aliasing among the correlated pairs or triplets. On the other hand, elastic as well as inelastic scattering will cause aliasing in a Rossi-alpha measurement.

\subsubsection{Inelastic scattering}

Inelastic scattering will produce all of the effects that elastic scattering produces. In addition, gamma rays are generated in the inelastic scattering process. One consequence of these gamma rays is that, unlike elastic scattering, the delay of the detection can be decreased. In addition, detection of the scattered neutron will be correlated with the detection of these gamma rays. Therefore inelastic scattering in any material in the detector or in the proximity of the detector will generate both $g g$ and $n g$ correlations. Proximity is relative to the distances from the source to detectors. Consequently, inelastic scattering will produce detector-detector correlations analogous to that produced by fission except that there will be no neutron-neutron pairs. This correlation is

$$
C_{23 \mid i}\left(\tau_{23}\right)=C_{g g \mid i}\left(\tau_{23}\right)+C_{g n \mid i}\left(\tau_{23}\right)+C_{n g \mid i}\left(\tau_{23}\right)
$$

where

$$
\begin{aligned}
& C_{g g \mid i}\left(\tau_{23}\right)=N_{i} \overline{\gamma_{i}\left(\gamma_{i}-1\right)} \epsilon_{g}^{2} \delta\left(\tau_{23}-\frac{d_{3}-d_{3}}{c}\right) \\
& C_{g n \mid i}\left(\tau_{23}\right)=N_{i} \overline{\gamma_{i}} \epsilon_{g} \epsilon_{n}\left(\tau_{23}-\frac{d_{2}}{c}\right) \chi_{i}\left(\tau_{23}-\frac{d_{2}}{c}\right) \\
& C_{n g \mid i}\left(\tau_{23}\right)=N_{i} \overline{\gamma_{i}} \epsilon_{g} \epsilon_{n}\left(\frac{d_{3}}{c}-\tau_{23}\right) \chi_{i}\left(\frac{d_{3}}{c}-\tau_{23}\right) .
\end{aligned}
$$


The spectrum of scattered neutrons $\chi_{i}(\tau)$ will depend on the incident neutron spectrum as well as the scattering material. The bicorrelation can be written

$$
\begin{aligned}
C_{234 \mid i}\left(\tau_{23}, \tau_{24}\right) & =C_{g g g \mid i}\left(\tau_{23}, \tau_{24}\right) \\
& +C_{g g n \mid i}\left(\tau_{23}, \tau_{24}\right)+C_{g n g \mid i}\left(\tau_{23}, \tau_{24}\right)+C_{n g g \mid i}\left(\tau_{23}, \tau_{24}\right)
\end{aligned}
$$

where the four terms are defined as

$$
\begin{aligned}
& C_{g g|i|}\left(\tau_{23}, \tau_{24}\right)=N_{i} \overline{\gamma_{i}\left(\gamma_{i}-1\right)\left(\gamma_{i}-2\right)} \epsilon_{g}^{3} \delta\left(\tau_{23}-\frac{d_{3}-\frac{d_{2}}{c}}{c}\right) \delta\left(\tau_{24}-\frac{d_{4-\frac{d_{2}}{c}}}{c}\right) \\
& C_{g g n \mid i}\left(\tau_{23}, \tau_{24}\right)=N_{i} \overline{\gamma_{i}\left(\gamma_{i}-1\right)} \epsilon_{g}^{2} \delta\left(\tau_{23}-\frac{d_{3}-d_{2}}{c}\right) \epsilon_{n}\left(\tau_{24}-\frac{d_{2}}{c}\right) \chi_{i}\left(\tau_{24}-\frac{d_{2}}{c}\right) \\
& C_{g n g \mid i}\left(\tau_{23}, \tau_{24}\right)=N_{i} \overline{\gamma_{i}\left(\gamma_{i}-1\right)} \epsilon_{g}^{2} \delta\left(\tau_{24}-\frac{d_{4}-d_{2}}{c}\right) \epsilon_{n}\left(\tau_{23}-\frac{d_{2}}{c}\right) \chi_{i}\left(\tau_{23}-\frac{d_{2}}{c}\right) \\
& C_{n g g \mid i}\left(\tau_{23}, \tau_{24}\right)=N_{i} \overline{\gamma_{i}\left(\gamma_{i}-1\right)} \epsilon_{g}^{2} \int_{0}^{\infty} \epsilon_{n}\left(\tau_{12}\right) \chi_{i}\left(\tau_{12}\right) \delta\left(\tau_{23}+\tau_{12}-\frac{d_{3}}{c}\right) \\
& \cdot \delta\left(\tau_{24}+\tau_{12}-\frac{d_{4}}{c}\right) d \tau_{12} .
\end{aligned}
$$

There will be no $n n$ pairs or $n n n$ triplets produced by inelastic scattering of single neutrons alone.

The detector-detector correlation from inelastic scattering was simulated in MCNPPOLIMI. $[27,28]^{4} \quad$ A small cylindrical lead target was placed between two large detectors. The lead target was $1 \mathrm{~cm}$ in diameter $50 \mathrm{~cm}$ long. The distance between the detectors was $50.6 \mathrm{~cm}$. The detectors were $5 \mathrm{~cm}$ thick with a $50 \mathrm{~cm}$ square face. A $14 \mathrm{MeV}$ neutron pencil beam impinged on the target at right angles with the line between the two detectors on one end of the cylindrical target. This geometry maximized the probability of a neutron interaction in the target and also minimizes the probability that a gamma ray will interact in the target. The resulting corelation from one-million source neutrons is shown in Figure 18.

\footnotetext{
${ }^{4}$ The Monte Carlo code MCNP4C and the derivative MCNP-DSP[47] do not preserve the number of gamma rays generated by the inelastic scatter of neutrons.[28]
} 


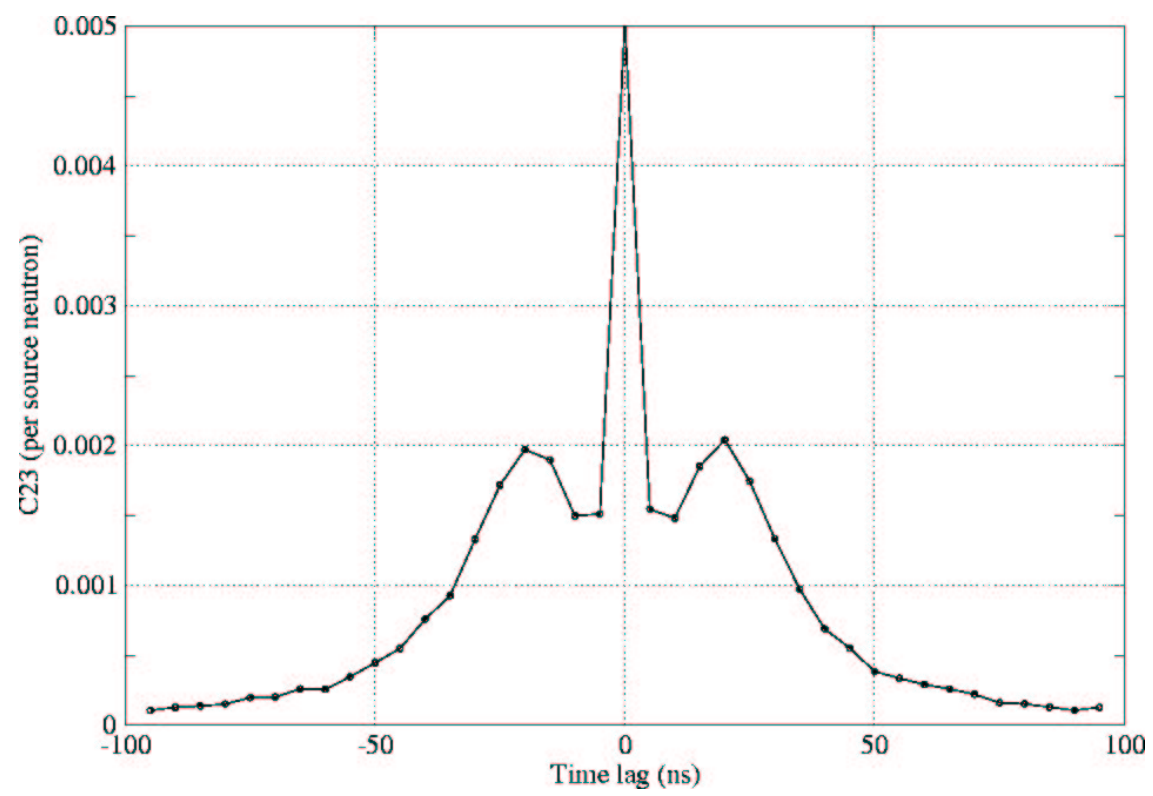

Figure 18: Cross correlations from the inelastic scattering of $14 \mathrm{MeV}$ neutrons on a lead target between two detectors.

\subsubsection{Alpha-n $(\alpha, n)$ reactions}

Neutrons produced by $(\alpha, n)$ reactions in nuclear materials can be considerable if the chemical composition is in the form of $\mathrm{PuO}_{2}, \mathrm{PuF}_{4}, U O_{2}$, or $U F_{6}$. The isotopic composition of the oxide or fluoride will also greatly influence the $(\alpha, n)$ reaction rate. All of the uranium and plutonium isotopes are alpha emitters. The $(\alpha, n)$ reaction rate will be greater if the isotopic composition is rich in isotopes with a high specific alpha activity.

These $(\alpha, n)$ reactions are accompanied by gamma rays. These gamma rays can arise from several sources. First, although generally very low in probability, the alpha decay may be accompanied by gamma emission. Second, the target nuclei may be left in an excited state after the reaction with the alpha particle resulting in the emission of one or more gamma rays along with the neutron. In addition, the neutron emitted may generate gamma rays through inelastic scattering. All of these sources of gamma rays will produce detector-detector correlations between the neutrons and gamma rays.

The correlations produced by $(\alpha, n)$ are completely analogous to those produced by inelastic scattering with the appropriate neutron spectrum, $\chi_{\alpha}(\tau)$ and gamma ray multiplicity 
$\gamma_{\alpha}$ substituted. As with inelastic scattering, there will be few $n n$ pairs. ${ }^{5}$

An experiment was performed to demonstrate the detector-detector correlation produced by an $(\alpha, n)$ source. The source was a Pu-Be source. The source contained $79.67 \mathrm{~g}$ of plutonium with an isotopic composition shown in Table 8 . Neutrons are generated by the reaction

$$
{ }^{9} \mathrm{Be}+{ }^{4} \alpha \rightarrow{ }^{12} \mathrm{C}+{ }^{1} n+5.708 \mathrm{MeV} .
$$

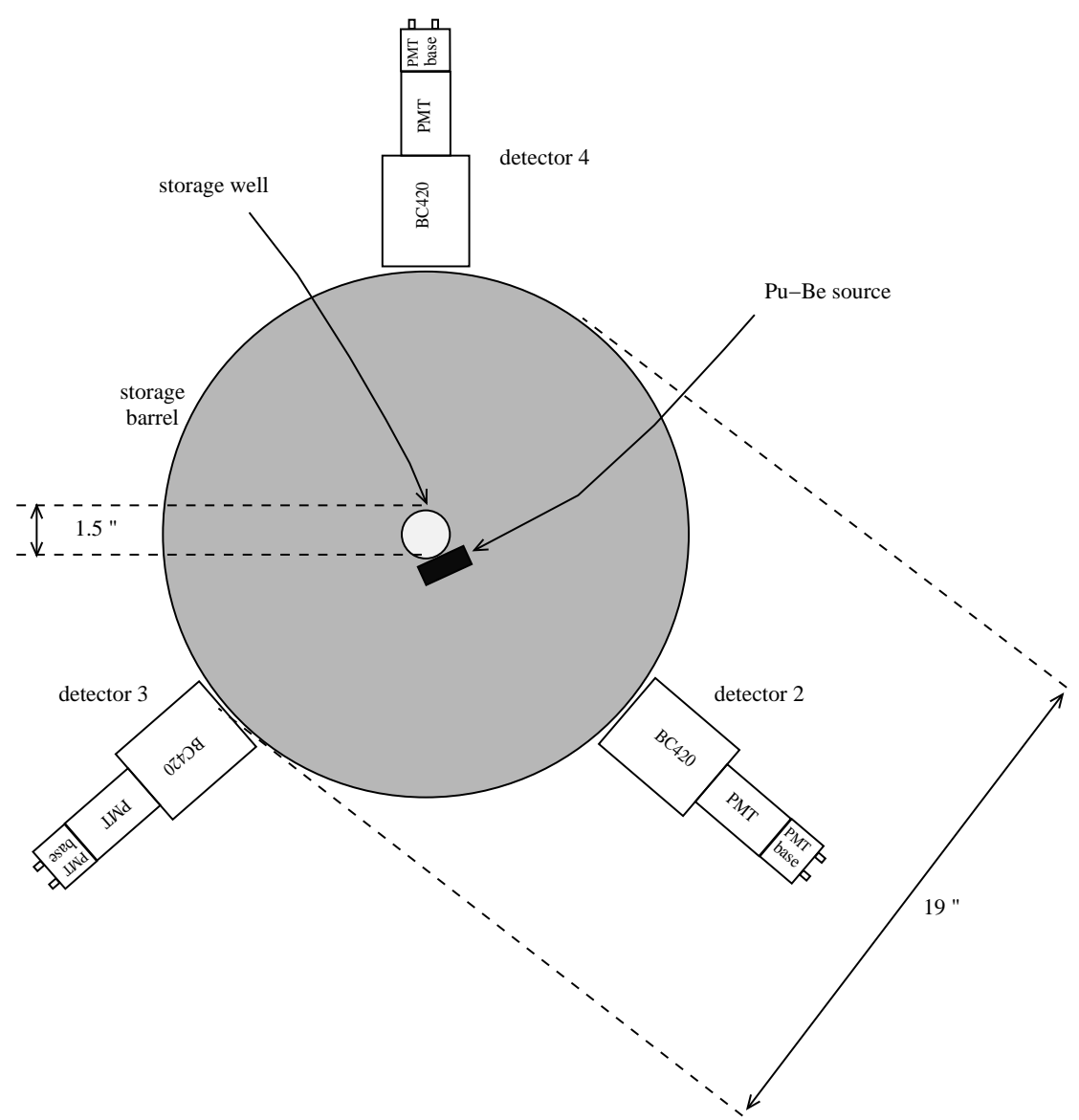

Figure 19: Experimental setup for the Pu-Be experiment.

The threshold of the reaction is about $1 \mathrm{MeV}$. The ${ }^{12} \mathrm{C}$ can be in the ground state or a $4.43 \mathrm{MeV}$ excited state accompanied by a $4.43 \mathrm{MeV}$ gamma ray or $7.65 \mathrm{MeV}$ excited state accompanied by a $4.43 \mathrm{MeV}$ and a $3.22 \mathrm{MeV}$ gamma ray typically. The probability of the second excited level increases rapidly for alpha rays above $4 \mathrm{MeV}$. According to

\footnotetext{
${ }^{5} \mathrm{~A}$ small number of neutron pairs can be produced through (n, 2n) reactions.[14]
} 
documentation on the Pu-Be source it produced nearly $5 \times 10^{6} \mathrm{n} / \mathrm{s}$. Of these only $2 \times 10^{-4}$ are from the spontaneous fission of ${ }^{240} \mathrm{Pu}$ and ${ }^{242} \mathrm{Pu} .{ }^{6}$

Table 8: Isotopic composition of the $\mathrm{Pu}-\mathrm{Be}$ source

\begin{tabular}{cr}
\hline Isotope & \% by mass \\
\hline${ }^{239} \mathrm{Pu}$ & 89.708 \\
${ }^{240} \mathrm{Pu}$ & 9.249 \\
${ }^{242} \mathrm{Pu}$ & 1.043 \\
\hline
\end{tabular}

The $\mathrm{Pu}$-Be source was placed about $2 \mathrm{~cm}$ from the center of a drum on top of the moderating material ${ }^{7}$ which was $77.5 \mathrm{~cm}$ above the floor. The geometry of the setup is shown in Figure 19. The asymmetry from not centering the source can be seen in the cross correlations in Figure 20. Three, 4-inch detectors were placed around the drum in an equilateral triangle at the level of the source. The center of the detector was $79.5 \mathrm{~cm}$ above the floor. The distance between the detectors was $43 \mathrm{~cm}$. The distance from each detector to the center of the drum was $25 \mathrm{~cm}$.

\subsubsection{Combined reactions}

In the previous subsections, each interaction was treated in isolation. In reality the interactions can occur in sequence. An example might be a fission followed by the inelastic scatter of a neutron. This combination of reactions is shown schematically in Figure 21. The time of fission is designated at time zero. Detector 2 detects a gamma from the fission at time $\tau_{a}$. A neutron from the fission undergoes an inelastic scatter at time $\tau_{b}$. This same neutron is then detected in detector $3, \tau_{c}$ after the scatter. A gamma ray from the inelastic scatter is then detected in detector $4, \tau_{d}$ after the inelastic scatter. The times of each of

\footnotetext{
${ }^{6}$ The specific neutron emissions from spontaneous fission from these isotopes are given in Section 2.2 in Table 1 .

${ }^{7}$ The moderating material was water equivalent polyester.
} 


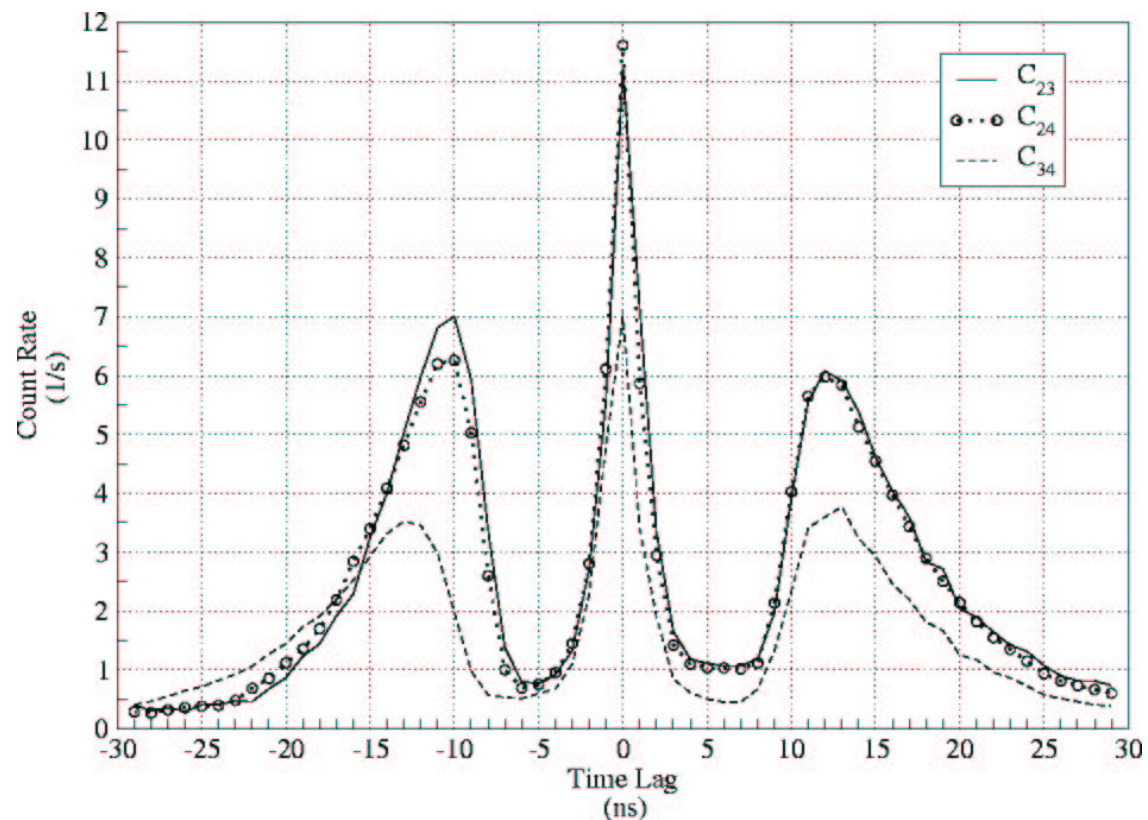

Figure 20: Cross correlations from a $\mathrm{Pu}-\mathrm{Be}$ source.

the detections is as follows:

$$
\begin{aligned}
& \tau_{12}=\tau_{a} \\
& \tau_{13}=\tau_{b}+\tau_{c} \\
& \tau_{14}=\tau_{b}+\tau_{d} .
\end{aligned}
$$

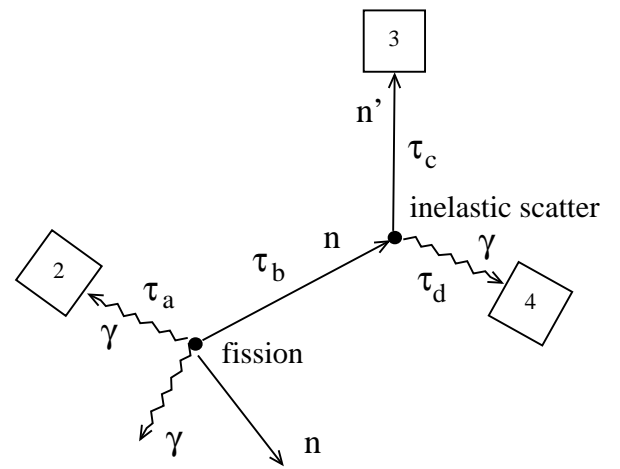

Figure 21: Fission followed by an inelastic scatter of a neutron. 
These detections would appear at the following location in the $C_{234}\left(\tau_{23}, \tau_{24}\right)$ correlation:

$$
\begin{aligned}
& \tau_{23}=\tau_{13}-\tau_{12}=\tau_{b}+\tau_{c}-\tau_{a} \\
& \tau_{24}=\tau_{13}-\tau_{12}=\tau_{b}+\tau_{d}-\tau_{a} .
\end{aligned}
$$

Note that $\tau_{b}-\tau_{a}$ appears in both time axes. This portion of the delay therefore appears on the $\tau_{23}=\tau_{24}$ diagonal. 


\section{CHAPTER V}

\section{NEUTRON MULTIPLICATION}

In this chapter the effects of neutron multiplication on FMD will be described. In Appendix A.3 the multiplicity moments resulting from multiplication are derived using probability generating functions. In this chapter the moments will be presented in a more intuitive fashion. In addition the effect on the time distribution of the correlations will be described. First the treatment of multiplication in traditional shift register analysis and TIA will be described.

Multiplication effects are handled in the traditional shift-register analysis through the super-fission approach developed by Böhnel.[6] This approach treats the multiplication process as occurring instantaneously with the initiating fission event. This approach is satisfactory when the duration of the multiplication process is short compared to the detector system response time, or when all of the time variables are integrated out. Both conditions apply in the case of shift register analysis.

According to Böhnel the non-multiplying factorial moments can be replaced with the multiplying version as follows:

$$
\begin{aligned}
\bar{\nu} & \longrightarrow M \nu_{s 1} \\
\overline{\nu(\nu-1)} & \longrightarrow M^{2}\left[\nu_{s 2}+\left(\frac{M-1}{\nu_{i 1}-1}\right) \nu_{s 1} \nu_{i 2}\right] \\
\overline{\nu(\nu-1)(\nu-2)} & \longrightarrow M^{3}\left[\nu_{s 3}+\left(\frac{M-1}{\nu_{i 1}-1}\right)\left[3 \nu_{s 2} \nu_{i 2}+\nu_{s 1} \nu_{i 3}\right]\right. \\
& \left.+3\left(\frac{M-1}{\nu_{i 1}-1}\right)^{2} \nu_{s 1} \nu_{i 2}^{2}\right]
\end{aligned}
$$

where $\nu_{s n}, \nu_{i n}$ are the $\mathrm{n} t h$ reduced factorial moments of the spontaneous and induced fission distributions respectively as used in Equation 6. For example $\nu_{s 2}$ is merely $\overline{\nu_{s}\left(\nu_{s}-1\right)}$. 
Equation (92) is derived in Appendix A.3 using probability generating functions as Böhnel did.

These multiplying factorial moments are used in shift register analysis as was seen in Equation (6) and also replace the factorial moments in Equations (10) and (12) in TIA when the fission process is fast compared to the detector die-away time.

The leakage multiplication factor $M$ is defined as follows. If a neutron is introduced into a fissile system with a probability $p$ of inducing a fission, there will be $p \overline{\nu_{i}}$ neutrons after the first fission on average. Each of these neutrons has a probability $p$ of inducing another fission and producing $\overline{\nu_{i}}$ more neutrons. The number of neutrons after an infinite number of generations is then

$$
m=\sum_{n=0}^{\infty}\left(p \overline{\nu_{i}}\right)^{n}=\frac{1}{1-p \overline{\nu_{i}}}
$$

The product $p \overline{\nu_{i}}$ is designated as $k_{e f f}$. If the probability that a neutron leaks from the system is $1-p$, then the leakage multiplication is

$$
M=\frac{1-p}{1-p \overline{\nu i}}=\frac{1-p}{1-k_{e f f}} .
$$

The leakage multiplication factor $M$ also comes from the derivation of the multiplying factorial moments derived in Appendix A.3. See specifically Equation (157). ${ }^{1}$

\section{$\underline{5.1}$ The time evolution of the multiplication process}

In TIA the time variables are not integrated out. Therefore, if the duration of the multiplication process is comparable or long compared with the detector die-away time, the super-fission approach is not applicable. In this case, Baeten makes the reasonable assumption that the neutron population evolves according to the differential equation

$$
\frac{d n}{d t}=\alpha n
$$

where $\alpha=\frac{1-k_{e f f}}{l}$ is the Rossi-alpha and $l$ is the mean neutron lifetime. This equation comes from the simple point reactor model. The higher-order time correlations are built up

\footnotetext{
${ }^{1}$ This definition of the leakage multiplication assumes that the probability that a neutron is captured through a non-fission event $p_{c}$ is negligible. If $p_{c}$ is not negligible, an additional unknown is introduced. The effect is derived in Appendix A.5
} 
from this model. ${ }^{2} \quad$ At all times, the amplitude (independent of time) is separable from the exponential time distribution in the point reactor derivations.

The solution to Equation (95) is

$$
n(\tau)=\frac{e^{-\alpha \tau}}{l}
$$

As was shown in Section 3.2 the the probability time distribution $p(\tau)$ can be separated from the amplitude of the correlation. This probability density in the case of Equation (96) is

$$
p(\tau)=\alpha e^{-\alpha \tau} .
$$

A graph of this probability density for $M=1.2$ and $\alpha=\frac{1}{4 n s}$ is shown in Figure 22. This probability density will be used as an example throughout this section. The probability $p(\tau)$ is not restricted to an exponential as in this example however.

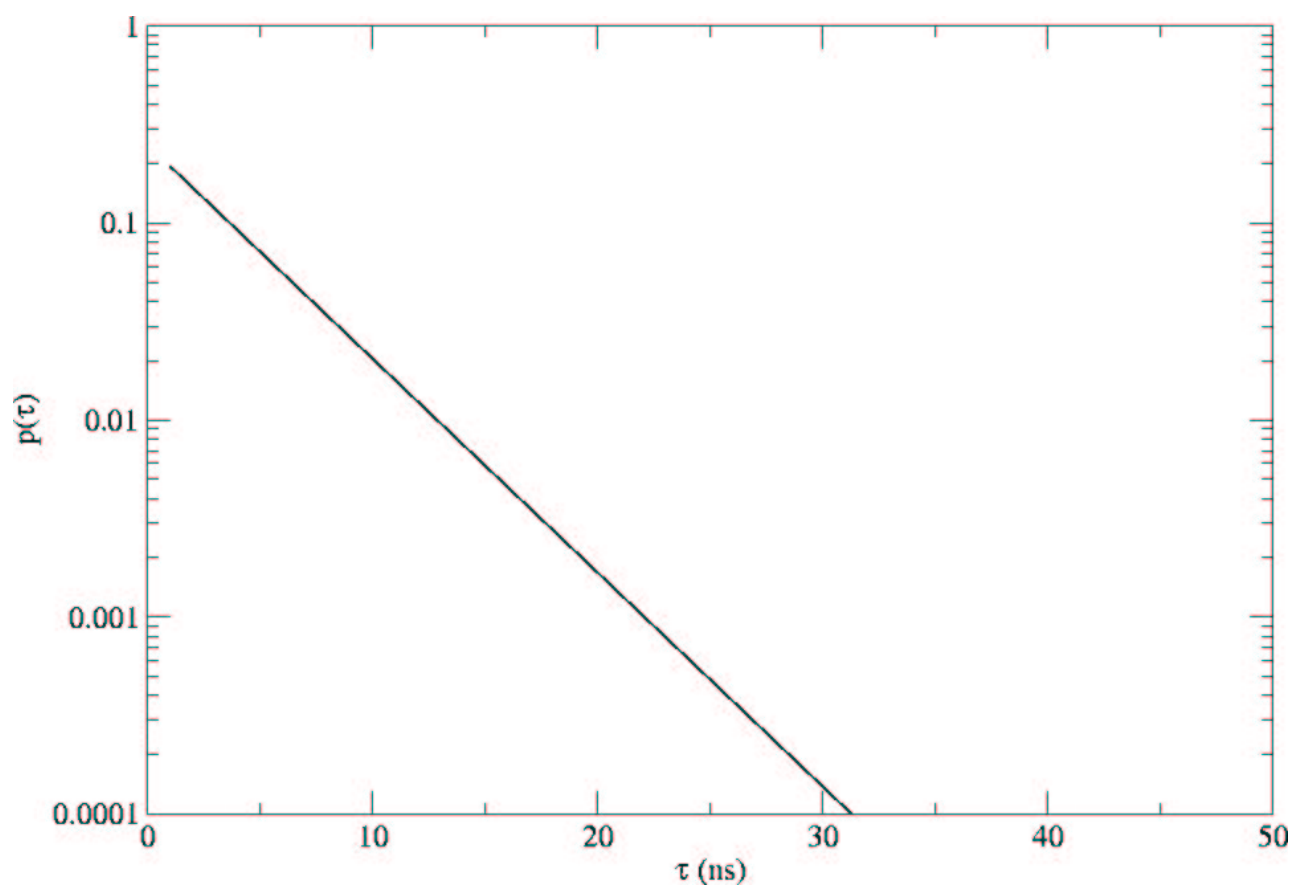

Figure 22: Probability of induced fission: $p(\tau)=\alpha e^{-\alpha t}$.

Assume that the time of a detection $\tau_{d}$ after a fission is a random variable and has the

\footnotetext{
${ }^{2}$ See Reference [1] at page 90. Mattingly derived similar equations using the point reactor model [30].
} 
probability density $p_{d}\left(\tau_{d}\right)$ where $p_{d}\left(\tau_{d}\right)=\epsilon_{\gamma} \delta\left(\tau_{d}\right)+\epsilon_{n}\left(\tau_{d}\right) \chi\left(\tau_{d}\right)$. This probability density, which was developed in Section 3.5 and Chapter 4, is shown in Figure 23. Assume, in addition, that the time of an induced fission after a spontaneous fission $\tau_{f}$ is a random variable with the probability density $p_{f}\left(\tau_{f}\right)$. For illustrative purposes it will be assumed that $p_{f}\left(\tau_{f}\right)$ is defined by Equation (97). The time of a detection of a fission $\tau$ following a spontaneous fission is then $\tau=\tau_{f}+\tau_{d}$ for fast recoil detectors. It is reasonable that $\tau_{f}$ and $\tau_{d}$ are independent. The probability density of a detection following a spontaneous fission is

$$
p(\tau)=\int_{0}^{\infty} p_{f}\left(\tau_{f}\right) p_{d}\left(\tau-\tau_{f}\right) d \tau_{f}
$$

This result is a well known property of the sum of independent random variables. ${ }^{3}$ A graph of this equation is shown in Figure 23. This probability density which can be decomposed into $p_{g}(\tau)$ and $p_{n}(\tau)$ is then used to calculate $C_{12}\left(\tau_{12}\right)$ as shown in Section 3.5. This correlation can then be used to derive the higher order correlations according to the method described in Section 4.1. The only difference is that the factorial moments used to determine the amplitude of the correlation must be replaced with the multiplying factorial moments which are derived in Appendix A and described in the Sections 5.2 to 5.4. Notice from Figure 23 that the gamma portion of the probability density has two distinct components. The first approximate delta function results from spontaneous fissions. The exponential portion comes from the induced fissions. The relative amplitude of these two components can be seen in the factorial moment in Equation (101) which will be described later in Section 5.3.

The probability density for the detector-detector correlation with multiplication is shown in Figure 24 along with the non-multiplying version. Each of the four components, $g g, g n$, $n g$ and $n n$, are shown along with the total. The dependencies and amplitude information is assumed to be contained in the factorial moments. Similarly the three-detector probability density with multiplication is shown in Figure 26. The eight regions for each triplet of neutron and gamma combinations are visible. For comparison, the non-multiplying version

\footnotetext{
${ }^{3}$ See Reference [13] at page 126 .
} 


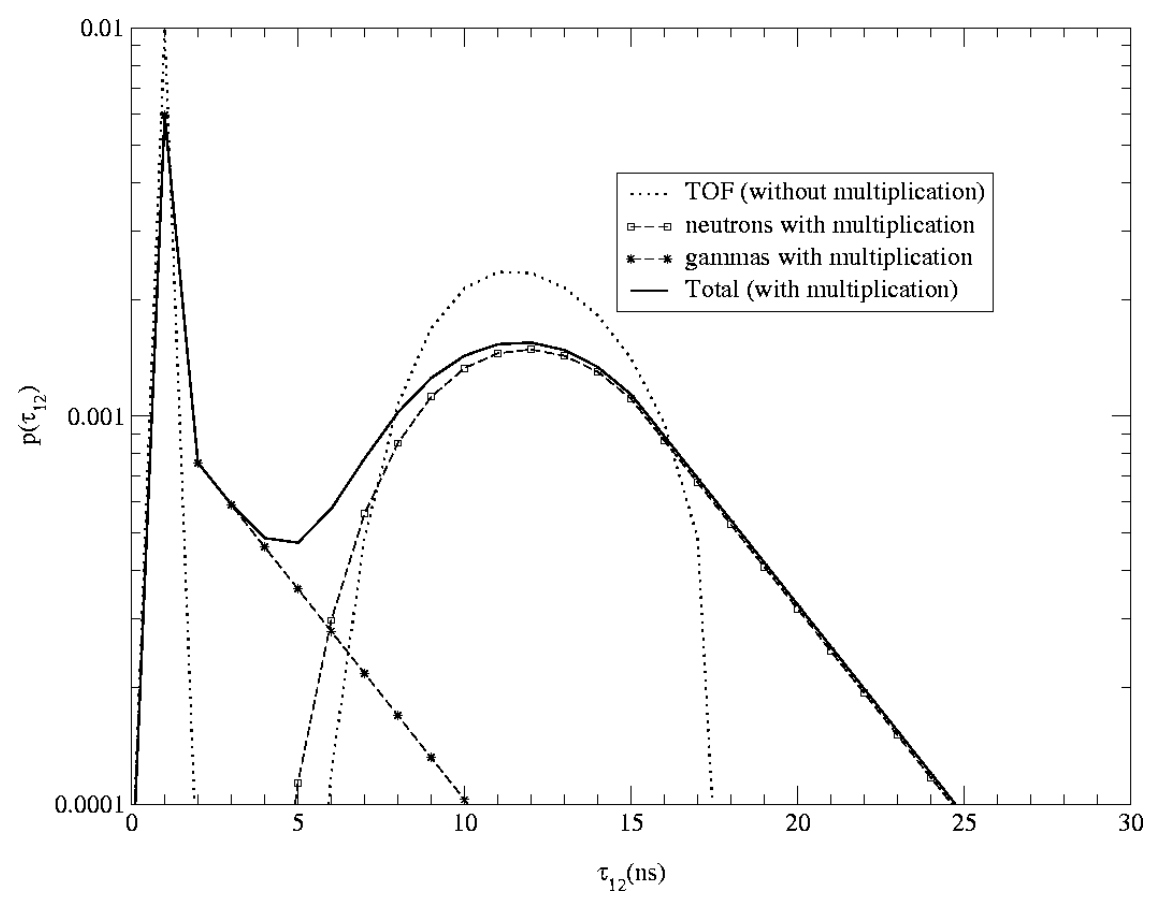

Figure 23: Theoretical $p_{12}\left(\tau_{12}\right)$ convolved with exponential fission distribution: $M=1.2, \alpha=\frac{1}{4 n s}$.

is shown in Figure 25.

\subsection{Neutron singles, doubles and triples}

The moments for neutron singles, doubles and triples have already been derived by Böhnel.[6] These moments are shown in Equation (92). The diagrams from reactor dynamics used by Mattingly [30] and Baeten [1] are shown in Figures 27, 28 and 29. The first dark circle represents a spontaneous fission. In the case of a single detection, the $\overline{\nu_{s}}$ neutrons from this spontaneous fission induce more fissions. This fission chain is represented by a solid line. Finally, of the neutrons generated by this process, one is detected. This detection is represented by an open circle.

There are an average of $\overline{\nu_{s}}$ neutrons from this spontaneous fission. If we call the average number of induced fissions $f_{i}$, there are $\nu_{i} f_{i}$ neutrons generated from induced fission. Of these, $1-p$ escape the system and are available for detection. Therefore,

$$
M \overline{\nu_{s}}=(1-p)\left[\overline{\nu_{s}}+f_{i} \overline{\nu_{i}}\right] .
$$




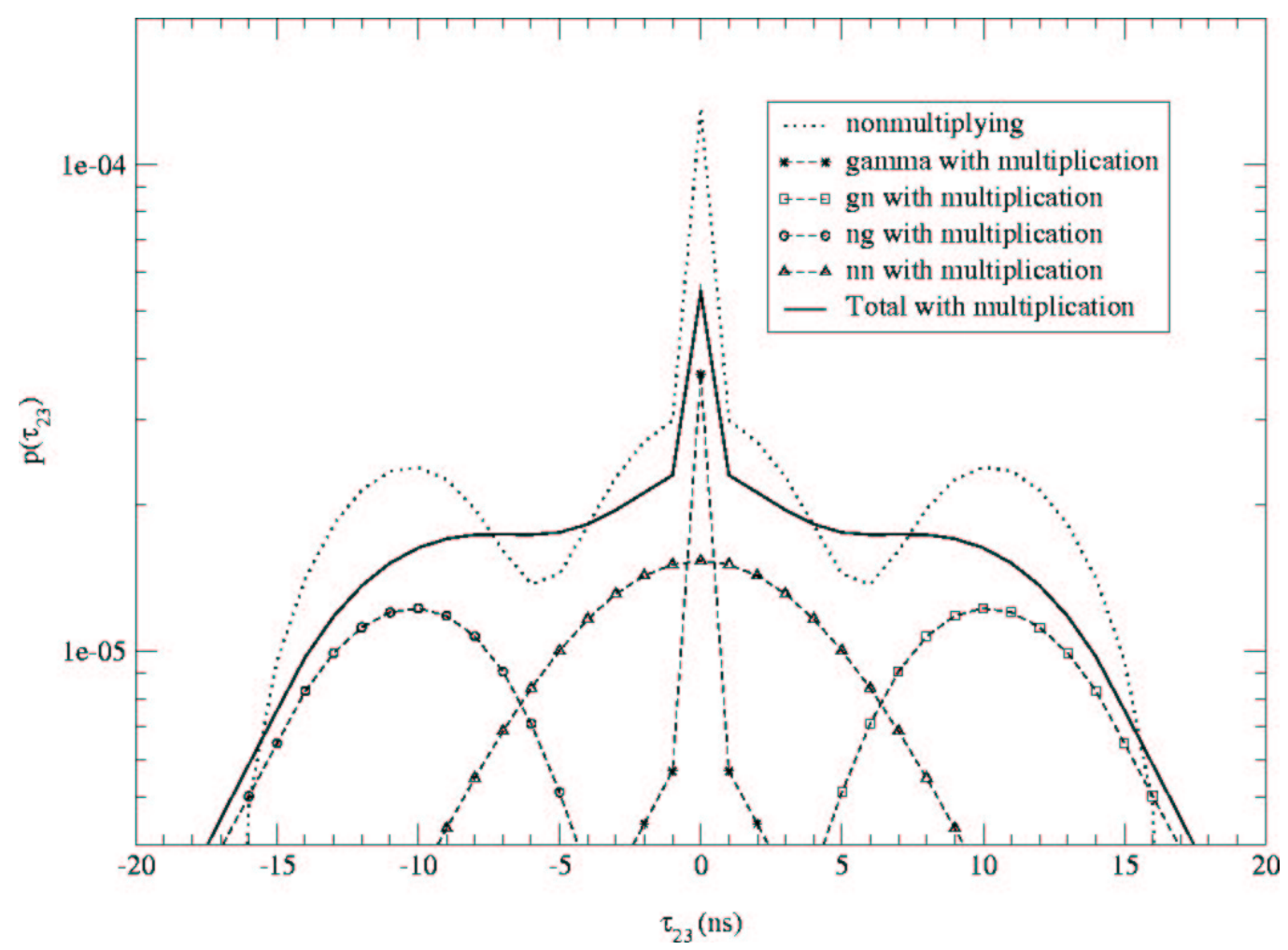

Figure 24: Comparison of theoretical $p_{23}\left(\tau_{23}\right)$ with and without multiplication: $M=1.2, \alpha=\frac{1}{4 n s}$.

From this equation, noting from Equation (94) that $p=\frac{M-1}{\nu_{i} M-1}$, the total number of induced fissions from a spontaneous fission is

$$
f_{i}=\frac{M-1}{\overline{\nu_{i}}-1} \overline{\nu_{s}}
$$

The diagram for a pair of neutron detections is shown in Figure 28. These two neutrons can be traced back to a single fission in the multiplication process. This parent fission can be either the spontaneous fission or an induced fission. Both of these cases can be seen in the figure. These two cases also produce two terms in the multiplicity moment as seen in the second line of Equation (92). In the case of double neutron detections the moment is proportional to the leakage multiplication squared. When the parent fission is the spontaneous fission, the moment is proportional to $\nu_{s}\left(\nu_{s}-1\right)$. When the parent fission is an induced fission, the moment is proportional to the number of fissions $f_{i}$ and $\nu_{i}\left(\nu_{i}-1\right)$.

Figure 29 shows the four combinations of triple neutron detections. As expected, the 


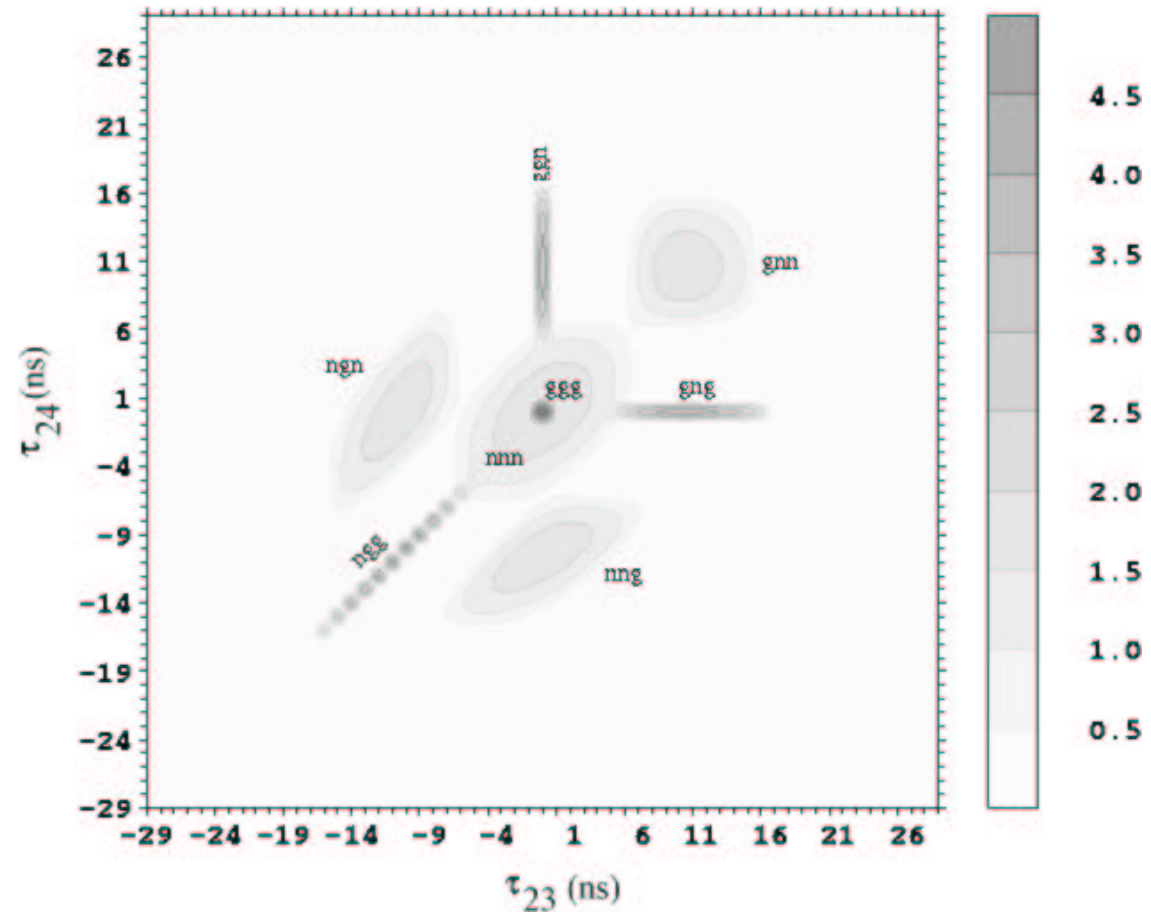

Figure 25: Theoretical $p_{234}\left(\tau_{23}, \tau_{24}\right)$ without multiplication. (amplitude is arbitrary)

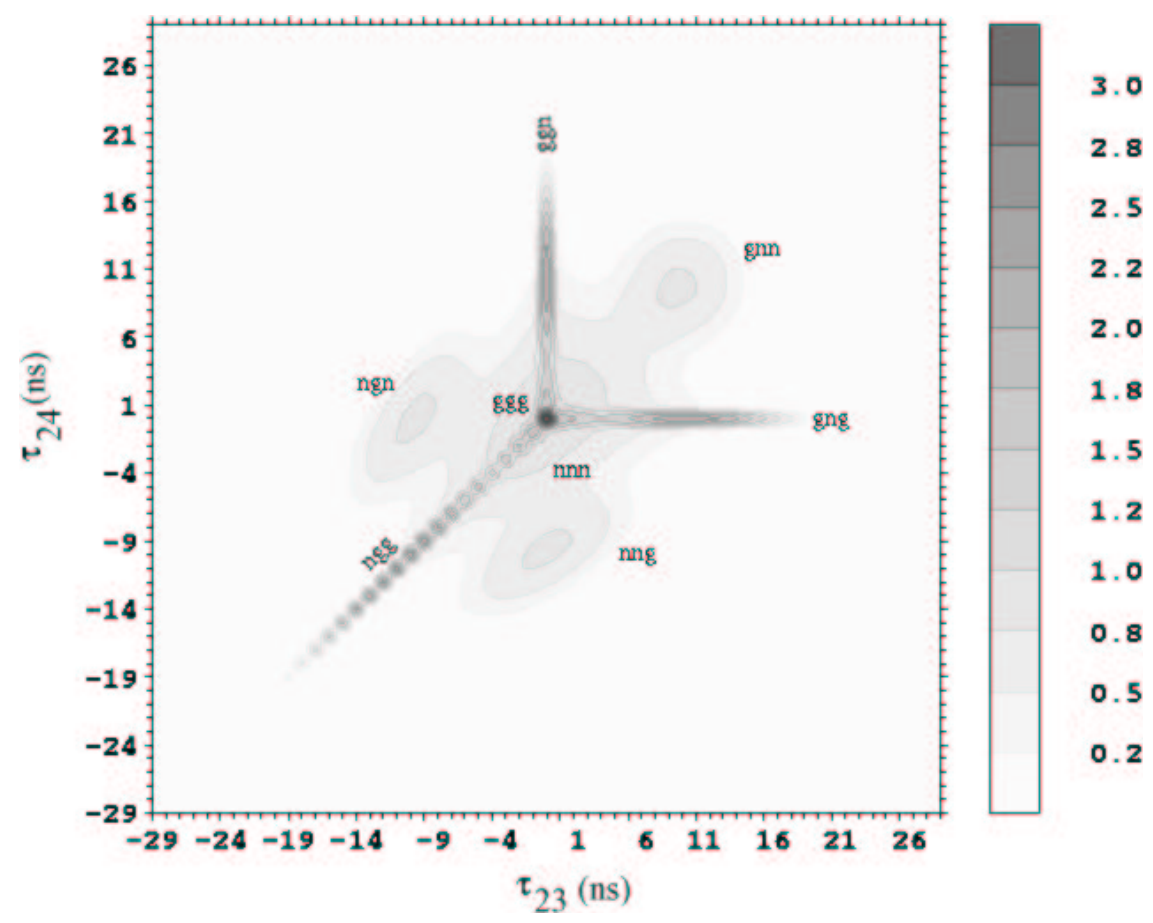

Figure 26: Theoretical $p_{234}\left(\tau_{23}, \tau_{24}\right)$ with multiplication: $M=1.2, \alpha=\frac{1}{4 n s}$. (amplitude is arbitrary) 


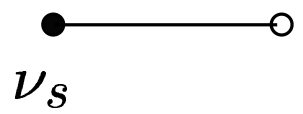

Figure 27: Neutron singles. The solid line represents a fission chain reaction process.
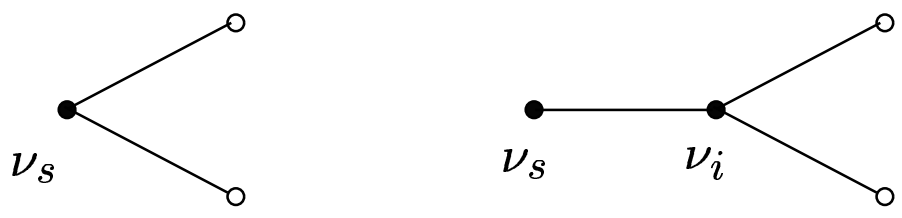

Figure 28: Neutron doubles.

third line of Equation (92) has four terms. The parent fission can be the same for all three neutrons or two neutrons can come from one parent fission and the third neutron from another fission. The parent fission can also be either a spontaneous fission or an induced fission. When all three neutrons arise from an induced fission, the multiplicity moment is $M^{3} \nu_{s}\left(\nu_{s}-1\right)\left(\nu_{s}-2\right)$. In the case of all three neutrons coming from a single induce fission, the multiplicity moment is $f_{i} M^{3} \nu_{i}\left(\nu_{i}-1\right)\left(\nu_{i}-2\right)$.
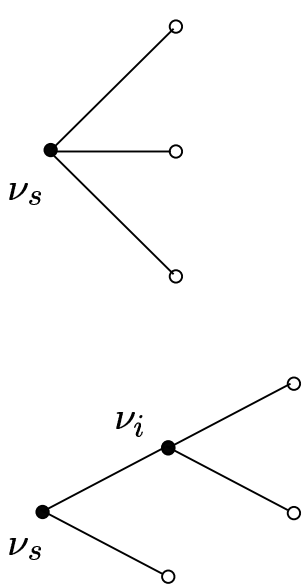
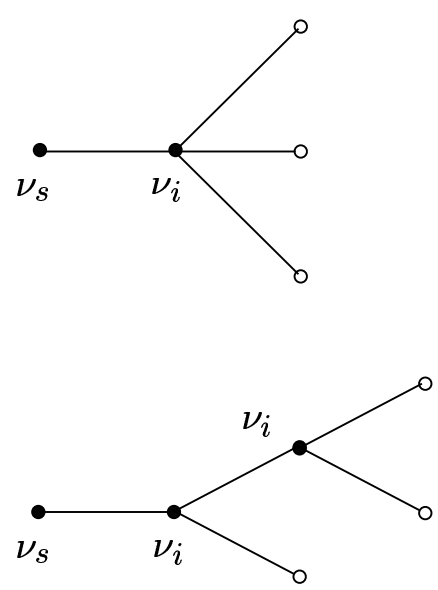

Figure 29: Neutron Triples.

The two fission parent cases can either involve a spontaneous fission or induced fission. In the case of two induced fissions, a $f_{i}^{2}$ factor is present. When one of the two fission parents is a spontaneous fission, the second reduced moments $\nu_{i}\left(\nu_{i}-1\right)$ and $\nu_{s}\left(\nu_{s}-1\right)$ are involved. Both of these cases have a factor of three in the moment. 
Why is there no case in Figure 29 involving two neutrons from a spontaneous fission and the third from an induced fission? This case reduces to three neutrons from spontaneous fission. Remember that each solid line represents a fission chain. The nodes are the common ancestor of the neutron.

\subsection{Gamma singles, doubles and triples}

The detection combinations for gamma rays in the fission multiplication process are shown in Figures 30, 31 and 32. The connection between the generation of a gamma ray by fission, represented by a solid circle, and its detection, represented by an open circle, is a dotted line. The dotted line indicates that this process, unlike a neutron, does not involve multiplication.

The two possible single gamma detections are shown in Figure 30. The gamma can come from the $\gamma_{s}$ gamma rays from a spontaneous fission or from the $\gamma_{i}$ gamma rays from $f_{i}$ induced fissions. The multiplicity moment for gamma rays should be replaced for multiplication as follows

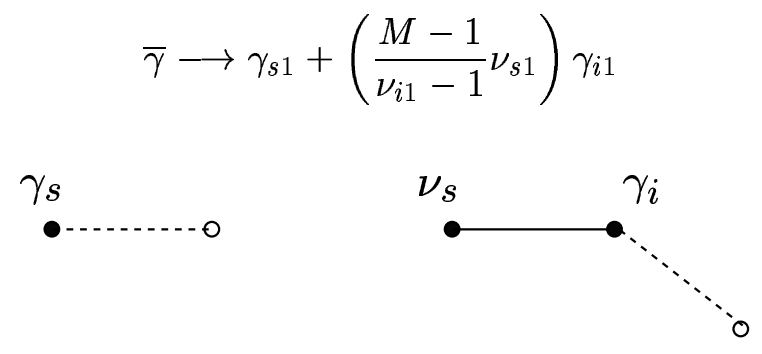

Figure 30: Gamma singles. The solid line represents a fission chain reaction process. The dotted line is a non-multiplying process.

The three combinations of gamma pairs are shown in Figure 31. The pair of gamma rays can come from the spontaneous fission, one of the $f_{i}$ induced fissions, or one from each.

The second reduced factorial moment for gamma rays should therefore be replaced by three terms as follows:

$$
\overline{\gamma(\gamma-1)} \longrightarrow \gamma_{s 2}+2\left(\frac{M-1}{\nu_{i 1}-1} \nu_{s 1}\right) \gamma_{s 1} \gamma_{i 1}+\left(\frac{M-1}{\nu_{i 1}-1} \nu_{s 1}\right)^{2} \gamma_{i 2}
$$



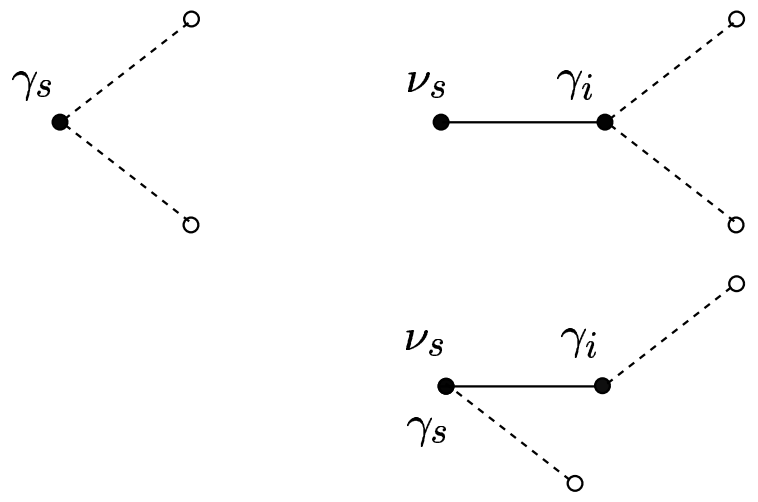

Figure 31: Gamma pairs.

The four combinations of gamma triplets are shown in Figure 32. The factorial moment for these triplets is

$$
\begin{aligned}
\overline{\gamma(\gamma-1)(\gamma-2)} & \longrightarrow \gamma_{s 3}+3\left(\frac{M-1}{\nu_{i 1}-1} \nu_{s 1}\right) \gamma_{s 2} \gamma_{i 1} \\
& +3\left(\frac{M-1}{\nu_{i 1}-1} \nu_{s 1}\right)^{2} \gamma_{s 1} \gamma_{i 2}+\left(\frac{M-1}{\nu_{i 1}-1} \nu_{s 1}\right)^{3} \gamma_{i 3}
\end{aligned}
$$
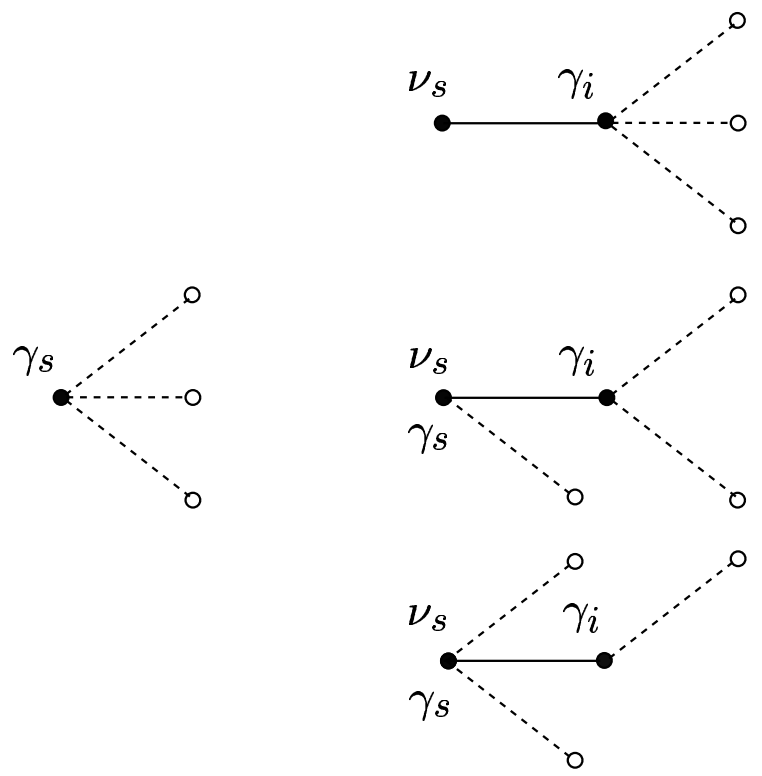

Figure 32: Gamma triples.

It should now be obvious how the four terms in Equation (103) relate to the four combinations in Figure 32. 


\subsection{Gamma-neutron combinations}

The gamma-neutron combinations, gn, ggn and gnn, are shown in Figures 33, 34 and 35 . As usual, there is a term for each combination. Fortunately, the moments are merely the product of the individual gamma and neutron moments from Sections 5.3 and 5.2. The factor of two in the $g n$ moment in Equation (104) represents that it is comprised of both $g n$ and $n g$. Similarly, the factor of three in the $g g n$ moment in Equation (105) represents the three combinations $g g n, g n g$ and $n g g$. The $g$ in the $g n n$ combination also has three positions as evident in the moment in Equation (106). The three combinations are separate and distinct in delay time as seen in Figures 14 and 15 in Chapter 4 and Figures 25 and 26 in this chapter.
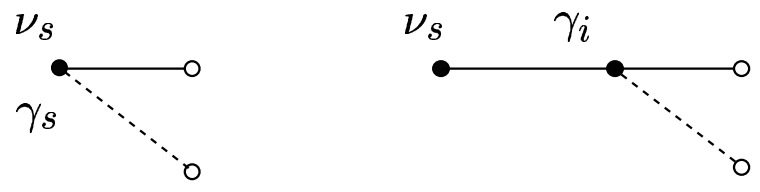

Figure 33: Gamma-neutron detection pairs.

$$
\overline{\nu \gamma} \longrightarrow 2 M \nu_{s 1}\left[\gamma_{s 1}+\left(\frac{M-1}{\nu_{i 1}-1} \nu_{s 1}\right) \gamma_{i 1}\right]
$$
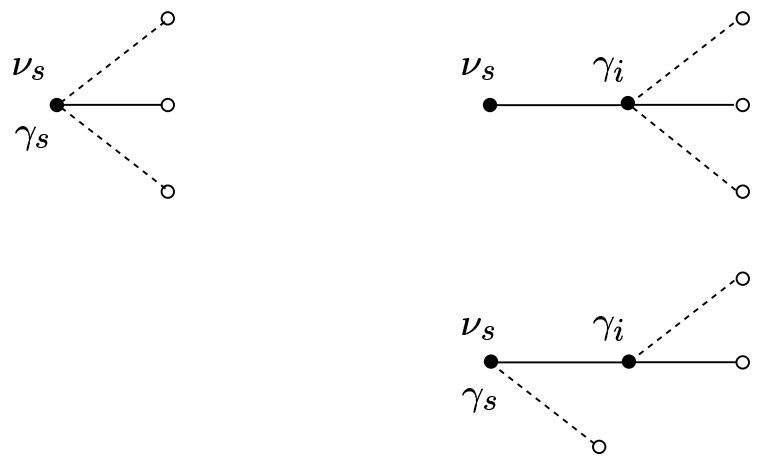

Figure 34: Gamma-gamma-neutron detection triples.

$$
\begin{aligned}
\overline{\gamma(\gamma-1)} \bar{\nu} & \longrightarrow 3 M \nu_{s 1}\left\{\gamma_{s 2}+2\left(\frac{M-1}{\nu_{i 1}-1} \nu_{s 1}\right) \gamma_{s 1} \gamma_{i 1}\right. \\
& \left.+\left(\frac{M-1}{\nu_{i 1}-1} \nu_{s 1}\right)^{2} \gamma_{i 2}\right\}
\end{aligned}
$$



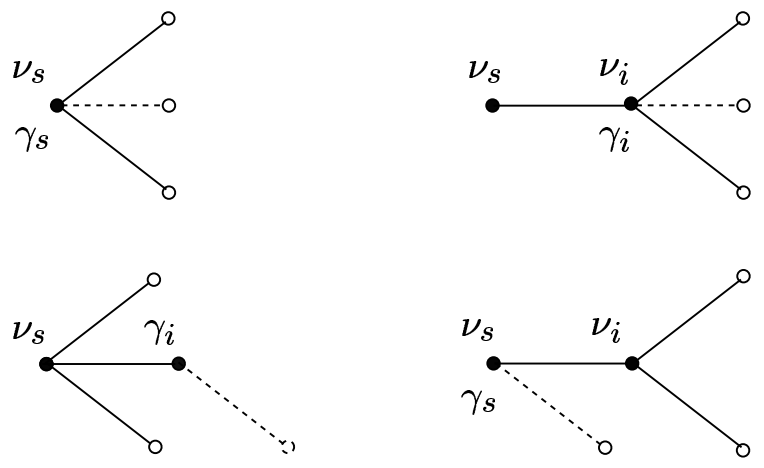

Figure 35: Gamma-neutron-neutron detection triples.

$$
\bar{\gamma} \overline{\nu(\nu-1)} \longrightarrow 3 M^{2}\left[\nu_{s 2}+\left(\frac{M-1}{\nu_{i 1}-1}\right) \nu_{s 1} \nu_{i 2}\right]\left\{\gamma_{s 1}+\left(\frac{M-1}{\nu_{i 1}-1} \nu_{s 1}\right) \gamma_{i 1}\right\}
$$




\section{CHAPTER VI}

\section{DETECTOR DEAD-TIME AND ALTERNATIVE}

\section{ANALYSIS}

Both the source and detector dead-time have a time dependent effect on NMIS correlations. Dead-time is determined primarily by the pulse width setting on the CFD. The sourcedetector correlation can easily be corrected for dead-time. ${ }^{1} \quad$ The detected correlation with dead-time is proportional to the probability of a detection at time $\tau$ after fission, $p(\tau)$, minus the probability that the detector is dead at $\tau$. The detector is dead if a detection precedes $\tau$ within the dead-time $\tau_{d}$. This probability can be written as

$$
p(\tau)=\int_{\tau-\tau_{d}}^{\tau} p^{\prime}(t \mid \tau) d t
$$

where $p^{\prime}(t \mid \tau)$ is the probability of a detection at time $t$ after a fission, including dead-time loss, given that a detection occurred at $\tau$. The probability of a detection at time $\tau$ after a fission, also including the effect of dead-time, is then

$$
p^{\prime}(\tau)=p(\tau)\left[1-\int_{\tau-\tau_{d}}^{\tau} p^{\prime}(t \mid \tau) d t\right]
$$

The true probability of detection $p(\tau)$ without dead-time is then

$$
p(\tau)=\frac{p^{\prime}(\tau)}{1-\int_{\tau-\tau_{d}}^{\tau} p^{\prime}(t \mid \tau) d t}
$$

Because the detection efficiency in these experiments is low, dead-time is not extremely important. Figure 36 shows a typical source-detector correlation along with the probability of dead-time loss from Equation 107. Because the probability of dead-time loss is much less than one (on the order of $10^{-3}$ ), the correction is inconsequential.

\footnotetext{
${ }^{1} \mathrm{~A}$ similar analysis is performed in reference [54].
} 


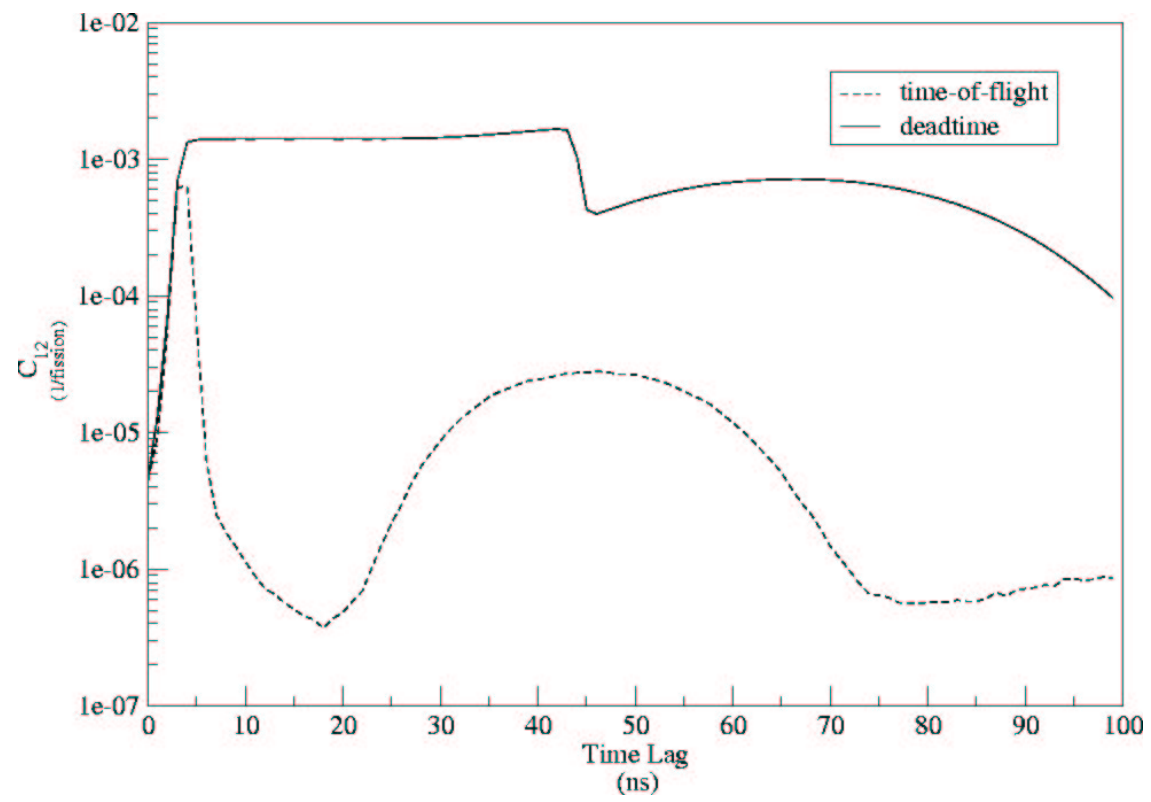

Figure 36: Source-detector correlation along with the probability of dead-time loss from Equation 107.

The source in the above analysis can be thought of as perfectly efficient with no deadtime. Such a source is useful as an unobserved source in deriving detector-detector correlations. A real, observed source is also characterized by the above analysis even when dead-time is considered. The only provision is that the source is not multiplying as is typically the case in an observed source. Fissions in a non-multiplying source occur with a probability that is uniformly distributed in time. Therefore source detection dead-time reduces the probability of pair detection independent of time. It merely scales the correlation just as source detection efficiency does.

The effect of dead-time can also be seen in the autocorrelation of either a detector or the source. This observation is a convenient way to measure and characterize detector dead-time.[40]

As shown in Section 4.1, the detector-detector correlation can be derived from unobserved source detector correlations as

$$
\left.p_{23}\left(\tau_{23}\right)=\int_{-\infty}^{\infty} p_{12}\left(\tau_{12}\right) p_{13}\left(\tau_{23}+\tau_{12}\right) \mid \tau\right) d \tau_{12} .
$$

In terms of the dead-time correction from Equation 98 and assuming independence, the 
detector-detector correlation is

$$
p_{23}\left(\tau_{23}\right)=\int_{-\infty}^{\infty} \frac{p_{12}^{\prime}\left(\tau_{12}\right) p_{13}^{\prime}\left(\tau_{23}+\tau_{12}\right)}{\left(1-\int_{\tau_{12}-\tau_{d}}^{\tau_{12}} p^{\prime}(t) d t\right)\left(1-\int_{\tau_{23}+\tau_{12}-\tau_{d}}^{\tau_{23}+\tau_{2}} p^{\prime}(t) d t\right)} d \tau_{12} .
$$

In the detector-detector correlation, the source detector correlations are generally unobserved. Without the source correlations the dead-time correction cannot be made. A similar problem arises in the well counter.

Without dead-time, the correlation between the source and detector in NMIS is

$$
C_{12}=\bar{\gamma} \epsilon_{g} \delta\left(\tau-\frac{d}{c}\right)+\bar{\nu} \epsilon_{n}(\tau) \chi(\tau)
$$

Consider first the gamma rays. The probability of detecting a gamma ray is equal to the detection efficiency times the number of gamma rays, $\gamma \epsilon_{g}$, or $\bar{\gamma} \epsilon_{g}$ on average. Because of dead-time only one of the $\gamma$ gamma rays can be detected. Therefore the probability becomes $1-p(0)$, i.e., the probability of detecting not-zero gamma rays. From the binomial distribution this probability is

$$
1-\left(1-\epsilon_{g}\right)^{\gamma}=\gamma \epsilon_{g}-\frac{\gamma(\gamma-1)}{2} \epsilon_{g}^{2}+\frac{\gamma(\gamma-1)(\gamma-2)}{6} \epsilon_{g}^{3}-\ldots
$$

In the binomial expansion, there can be only $\gamma+1$ terms, and furthermore small values of $\epsilon_{g}$ make some of these inconsequential.

A formalism was developed by Van der Werf[52] to infer multiplicity in the case of $N$ detectors when a detector could register either a detection or miss from a multiplet event, i. e., two or more hits are indistinguishable from one hit. ${ }^{2} \quad$ Let $k$ be the number of $\gamma$ gamma rays which strike any of $N$ detectors. The probability of $k$ hits on $N$ detectors from $\gamma$ gamma rays is

$$
p_{N}(k \mid \gamma)=\left(\begin{array}{l}
\gamma \\
k
\end{array}\right)\left(N \epsilon_{g}\right)^{k}\left(1-N \epsilon_{g}\right)^{\gamma-k}
$$

Of the $k$ hits on the $N$ detectors, the probability that they are distributed over $n$ of the $N$ detectors is

$$
p_{N}(n \mid k)=\left(\begin{array}{c}
N \\
n
\end{array}\right) \frac{k !}{N^{k}} S_{k}^{(n)}
$$

\footnotetext{
${ }^{2}$ Additional discussion of the derivation can be found in reference [8] at pages 35 and 75 .
} 
where $S_{k}^{(n)}$ is the Stirling numbers of the second kind. The Stirling number describes the number of ways a set with $k$ elements can be partitioned into $n$ disjoint, non-empty subsets. The probability of $n$ of $N$ detectors being triggered by $\gamma$ gamma rays is then the product of the foregoing:

$$
p_{N}(n \mid \gamma)=\left(\begin{array}{l}
\gamma \\
k
\end{array}\right) k ! \epsilon_{g}^{k}\left(1-N \epsilon_{g}\right)^{\gamma-k}\left(\begin{array}{c}
N \\
n
\end{array}\right) S_{k}^{(n)}
$$

In this form, the Stirling numbers can be tabulated in advance. Typically the equation is manipulated into the following form:

$$
p_{N}(n \mid \gamma)=\left(\begin{array}{c}
N \\
n
\end{array}\right) \sum_{i=0}^{n}(-1)^{n-i}\left(\begin{array}{c}
n \\
i
\end{array}\right)\left[1-(N-i) \epsilon_{g}\right]^{\gamma} .
$$

This formula has been extended to include cross-talk from scatter between detectors ${ }^{3}$

$$
p_{N}(n \mid \gamma)=\left(\begin{array}{c}
N \\
n
\end{array}\right) \sum_{i=0}^{n}(-1)^{n-i}\left(\begin{array}{c}
n \\
i
\end{array}\right)\left\{\left[1-(N-i) \epsilon_{g}\right]\left[1+f(1-N-i) \epsilon_{g}\right]\right\}^{\gamma}
$$

where $f$ is the probability that a radiation detected in one detector escapes and is detected in another detector. This correction for cross-talk will not be used here. First, the geometry of the experiments was chosen to minimize the cross-talk. Second, when cross-talk becomes significant, the time correlation characteristics will be useful in identifying it. ${ }^{4}$

Using Equation 117 for one detector, the probability of detecting zero of $\gamma$, gamma rays and one of $\gamma$, gamma rays is

$$
\begin{aligned}
p_{1}(0 \mid \gamma) & =\left(1-\epsilon_{g}\right)^{\gamma} \\
& =1-\epsilon_{g} \gamma+\frac{\gamma(\gamma-1)}{2 !} \epsilon_{g}^{2}-\frac{\gamma(\gamma-1)(\gamma-2)}{3 !} \epsilon_{g}^{3}+\ldots \\
p_{1}(1 \mid \gamma) & =1-\left(1-\epsilon_{g}\right)^{\gamma} \\
& =\epsilon_{g} \gamma-\frac{\gamma(\gamma-1)}{2 !} \epsilon_{g}^{2}+\frac{\gamma(\gamma-1)(\gamma-2)}{3 !} \epsilon_{g}^{3}+\ldots
\end{aligned}
$$

The second line of each equation is the binomial expansion.

\footnotetext{
${ }^{3}$ This formulation is typically used in physics experiments[38, 39].

${ }^{4}$ See Reference [43].
} 
For two detectors, the probability of detecting 0,1 or 2 gamma rays out of $\gamma$ emitted is

$$
\begin{aligned}
p_{2}(0 \mid \gamma) & =\left(1-2 \epsilon_{g}\right)^{\gamma} \\
& =1-2 \epsilon_{g} \gamma+\gamma(\gamma-1) 2 \epsilon_{g}^{2}-\gamma(\gamma-1)(\gamma-2) \frac{4}{3} \epsilon_{g}^{3}+\ldots \\
p_{2}(1 \mid \gamma) & =2\left[\left(1-\epsilon_{g}\right)^{\gamma}-\left(1-2 \epsilon_{g}\right)^{\gamma}\right] \\
& =2 \epsilon_{g} \gamma-\gamma(\gamma-1) 3 \epsilon_{g}^{2}+\gamma(\gamma-1)(\gamma-2) \frac{7}{3} \epsilon_{g}^{3}-\ldots \\
p_{2}(2 \mid \gamma) & =1+\left(1-2 \epsilon_{g}\right)^{\gamma}-2\left(1-\epsilon_{g}\right)^{\gamma} \\
& =\gamma(\gamma-1) \epsilon_{g}^{2}-\gamma(\gamma-1)(\gamma-2) \epsilon_{g}^{3}+\ldots
\end{aligned}
$$

For three detectors, the probability of detecting $0,1,2$ or 3 gamma rays out of $\gamma$ emitted is

$$
\begin{aligned}
p_{3}(0 \mid \gamma) & =\left(1-3 \epsilon_{g}\right)^{\gamma} \\
& =1-3 \epsilon_{g} \gamma+\gamma(\gamma-1) \frac{9}{2} \epsilon_{g}^{2}-\gamma(\gamma-1)(\gamma-2) \frac{9}{2} \epsilon_{g}^{3}+\ldots \\
p_{3}(1 \mid \gamma) & =3\left[\left(1-2 \epsilon_{g}\right)^{\gamma}-\left(1-3 \epsilon_{g}\right)^{\gamma}\right] \\
& =3 \epsilon_{g} \gamma-\gamma(\gamma-1) \frac{15}{2} \epsilon_{g}^{2}+\gamma(\gamma-1)(\gamma-2) \frac{19}{2} \epsilon_{g}^{3}+\ldots \\
p_{3}(2 \mid \gamma) & =3\left[\left(1-3 \epsilon_{g}\right)^{\gamma}-2\left(1-2 \epsilon_{g}\right)^{\gamma}+\left(1-\epsilon_{g}\right)^{\gamma}\right] \\
& =\gamma(\gamma-1) 3 \epsilon_{g}^{2}-\gamma(\gamma-1)(\gamma-2) 6 \epsilon_{g}^{3}+\ldots \\
p_{3}(3 \mid \gamma) & =1-\left(1-3 \epsilon_{g}\right)^{\gamma}+3\left(1-2 \epsilon_{g}\right)^{\gamma}-3\left(1-\epsilon_{g}\right)^{\gamma} \\
& =\gamma(\gamma-1)(\gamma-2) \epsilon_{g}^{3}-\gamma(\gamma-1)(\gamma-2)(\gamma-3) \frac{13}{12} \epsilon_{g}^{4}+\ldots
\end{aligned}
$$

After the detection of a gamma ray, the detector will be dead not only for subsequent gamma rays but also for the detection of neutrons for a period of $\tau_{d}$. The probability of this dead-time is equal to the probability of detecting a gamma $1-\left(1-\epsilon_{g}\right)^{\gamma} \approx \epsilon_{g} \gamma$. Practically, $\tau_{d}$ extends over a substantial portion of the neutron distribution. Furthermore, it can easily be extended to cover virtually all subsequent neutrons. To simplify the analysis it is assumed that $\tau_{d}$ covers virtually all subsequent neutrons. 
For a single detector

$$
\begin{aligned}
p(n) & =p_{1}(0 \mid \gamma) p_{1}(1 \mid \nu) \\
& =\left(1-\epsilon_{g}\right)^{\gamma}\left[1-\left(1-\epsilon_{n}\right)^{\nu}\right] \\
& \approx \epsilon_{n} \nu\left(1-\epsilon_{g} \gamma\right) \\
p(g) & =p_{1}(1 \mid \gamma) p_{0}(0 \mid \nu) \\
& =1-\left(1-\epsilon_{g}\right)^{\gamma} \\
& \approx \epsilon_{g} \gamma .
\end{aligned}
$$

These equations should satisfy one's intuition. A neutron can be detected only if no gamma is detected. In addition, $p_{0}(0 \mid \nu)=1$ can be thought of as the conditional probability $p_{1}(n \mid g)$. In other words if a gamma is detected in the only detector, the probability of detecting zero neutrons is a certainty.

The approximations in these equations come from keeping the first nonzero term from the binomial expansion. The approximation represents the case of very low detection efficiency and agrees with the probabilities for no dead-time for gamma rays.

Similar analysis can be extended to two and three detectors. For two detectors,

$$
\begin{aligned}
p(n, n) & =p_{2}(0 \mid \gamma) p_{2}(2 \mid \nu) \\
& =\left(1-2 \epsilon_{g}\right)^{\gamma}\left[1+\left(1-2 \epsilon_{n}\right)^{\nu}-2\left(1-\epsilon_{n}\right)^{\nu}\right] \\
& \approx\left(1-2 \epsilon_{g} \gamma\right) \nu(\nu-1) \epsilon_{n}^{2} \\
p(n, g) & =p_{2}(1 \mid \gamma) p_{1}(1 \mid \nu) \\
& =2\left[2\left(1-\epsilon_{g}\right)^{\gamma}-\left(1-2 \epsilon_{g}\right)^{\gamma}\right]\left[1-\left(1-\epsilon_{n}\right)^{\nu}\right] \\
& \approx 2 \epsilon_{g} \gamma \epsilon_{n} \nu \\
p(g, g) & =p_{2}(2 \mid \gamma) p_{0}(0 \mid \nu) \\
& =1+\left(1-2 \epsilon_{g}\right)^{\gamma}-2\left(1-\epsilon_{g}\right)^{\gamma} \\
& \approx \gamma(\gamma-1) \epsilon_{g}^{2} .
\end{aligned}
$$


For three detectors,

$$
\begin{aligned}
p(n, n, n) & =p_{3}(0 \mid \gamma) p_{3}(3 \mid \nu) \\
& =\left(1-3 \epsilon_{g}\right)^{\gamma}\left[1-\left(1-3 \epsilon_{n}\right)^{\nu}+3\left(1-2 \epsilon_{n}\right)^{\nu}-3\left(1-\epsilon_{n}\right)^{\nu}\right] \\
& \approx\left(1-3 \epsilon_{g} \gamma\right) \nu(\nu-1)(\nu-2) \epsilon_{n}^{3} \\
p(n, n, g) & =p_{3}(1 \mid \gamma) p_{2}(2 \mid \nu) \\
& =3\left[\left(1-2 \epsilon_{g}\right)^{\gamma}-\left(1-3 \epsilon_{g}\right)^{\gamma}\right]\left[1+\left(1-2 \epsilon_{n}\right)^{\nu}-2\left(1-\epsilon_{n}\right)^{\nu}\right] \\
& \approx 3 \epsilon_{g} \gamma \nu(\nu-1) \epsilon_{n}^{2} \\
p(n, g, g) & =p_{3}(2 \mid \gamma) p_{1}(1 \mid \nu) \\
& =3\left[\left(1-3 \epsilon_{g}\right)^{\gamma}-2\left(1-2 \epsilon_{g}\right)^{\gamma}+\left(1-\epsilon_{g}\right)^{\gamma}\right]\left[1-\left(1-\epsilon_{n}\right)^{\nu}\right] \\
& \approx \gamma(\gamma-1) 3 \epsilon_{g}^{2} \epsilon_{n} \nu \\
p(g, g, g) & =p_{3}(3 \mid \gamma) p_{0}(0 \mid \nu) \\
& =1-\left(1-3 \epsilon_{g}\right)^{\gamma}+3\left(1-2 \epsilon_{g}\right)^{\gamma}-3\left(1-\epsilon_{g}\right)^{\gamma} \\
& \approx \gamma(\gamma-1)(\gamma-2) \epsilon_{g}^{3} .
\end{aligned}
$$




\section{CHAPTER VII}

\section{EXPERIMENTAL DESCRIPTION}

The source-detector geometry for the experiments is shown in Figure 37. It is comprised of three plastic scintillating detectors arranged in an equilateral triangle around a ${ }^{252} C f$ source contained in an ionization chamber. The ion-chamber as shown in Figure 39 was pointed down with the plates parallel to the floor. The distance $s$ between the centers of the front face of the detectors, was $40 \mathrm{~cm}$. The radial distance from the source to the front face of each detector was $23 \mathrm{~cm}$. The bottom of the detectors was $33.5 \mathrm{~cm}$ above the surface of the table, which in turn was $88.7 \mathrm{~cm}$ above the floor. The ${ }^{252} \mathrm{Cf}$ source is intended to represent the unknown sample to be assayed such as plutonium. In practice, the sample would not be contained in an ion-chamber. The purpose of the ion-chamber is to provide additional timing information which is not available in standard active and passive correlation measurements.

The time bins were set to 1 ns. The correlation window was set to 512 ns.

\subsection{Functional block diagram}

Figure 38 shows a functional block diagram of the experimental setup. The three detectors were Bicron Model BC-420 fast plastic scintillating detectors and are described more fully in Section 7.2. The high voltage (HV) for each detector was set so that the full energy deposition from a $1 \mathrm{MeV}$ neutron produced a $40 \mathrm{mV}$ pulse which corresponds to the threshold setting in the constant fraction discriminators (CFDs). The CFDs are ORTEC 935 Quad channel CFDs. The output of the photomultiplier tubes were connected to the input of the CFDs. The CFDs produce a zero to negative $800 \mathrm{mV}$ transition at the output to mark the time of a detection event. This output is sent to the NMIS data acquisition board, also called the Data Acquisition/Data Capture and Compression (DA/DCC) board, which 


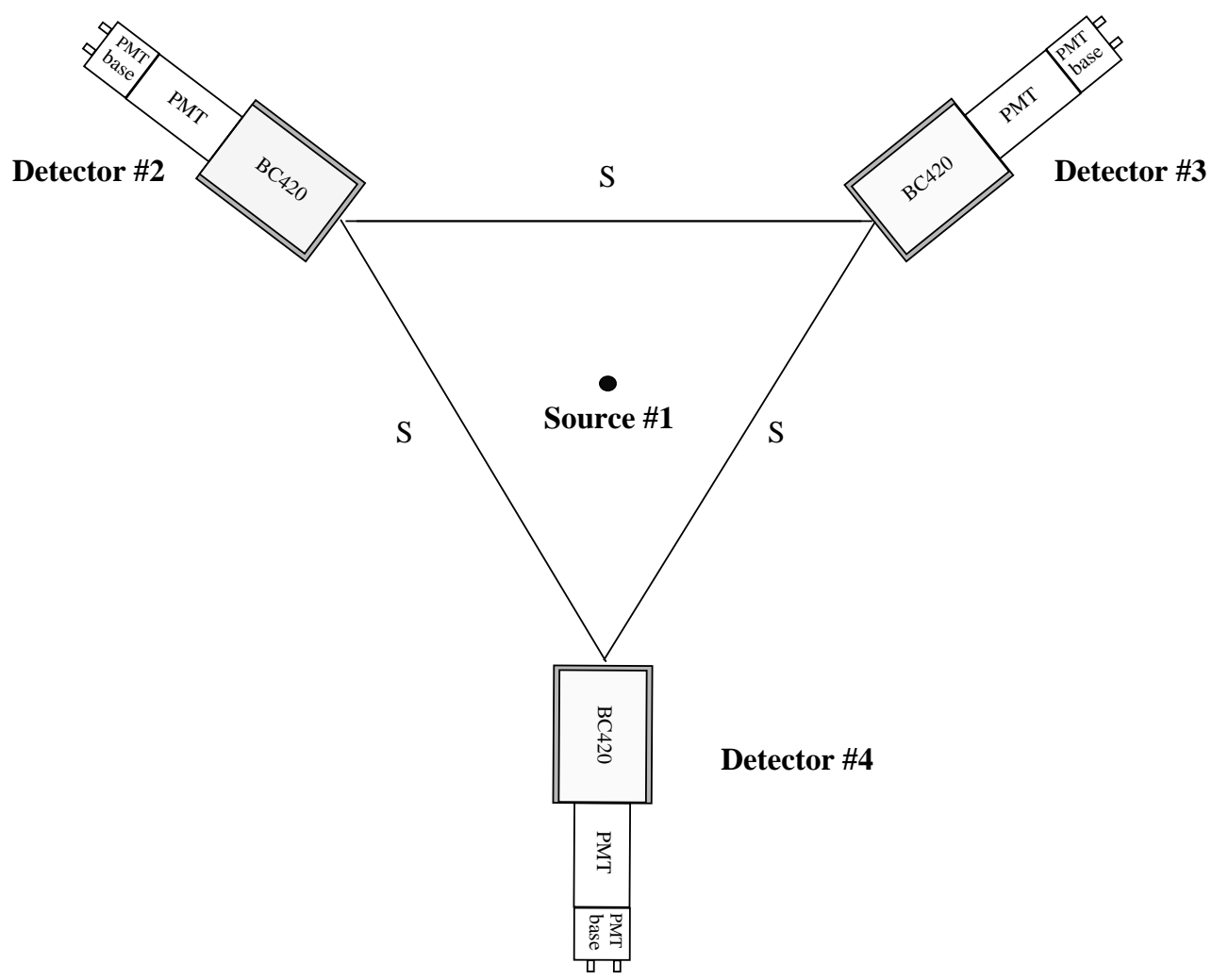

Figure 37: Experimental setup.

is installed in a personal computer. The software computes the time correlations between detection events. An intuitive description of this computation is given in Section 3.1. These correlations include the source with each detector $\left(C_{12}, C_{13}\right.$, and $\left.C_{14}\right)$, each pair of detectors $\left(C_{23}, C_{24}\right.$, and $\left.C_{34}\right)$, the source and each pair of detectors $\left(C_{123}, C_{124}\right.$, and $\left.C_{134}\right)$ and the correlation between the three detectors $\left(C_{234}\right)$.

\section{$\underline{7.2 \quad \text { Detectors }}$}

The detectors are Bicron BC-420 fast plastic scintillating detectors. Technical specifications for the detectors are listed in Table 9. A quarter inch of lead shielding was placed on the front face and sides of the detectors.

The multiplicity data was acquired with two sets of detectors, a 3-inch set and a 4-inch 


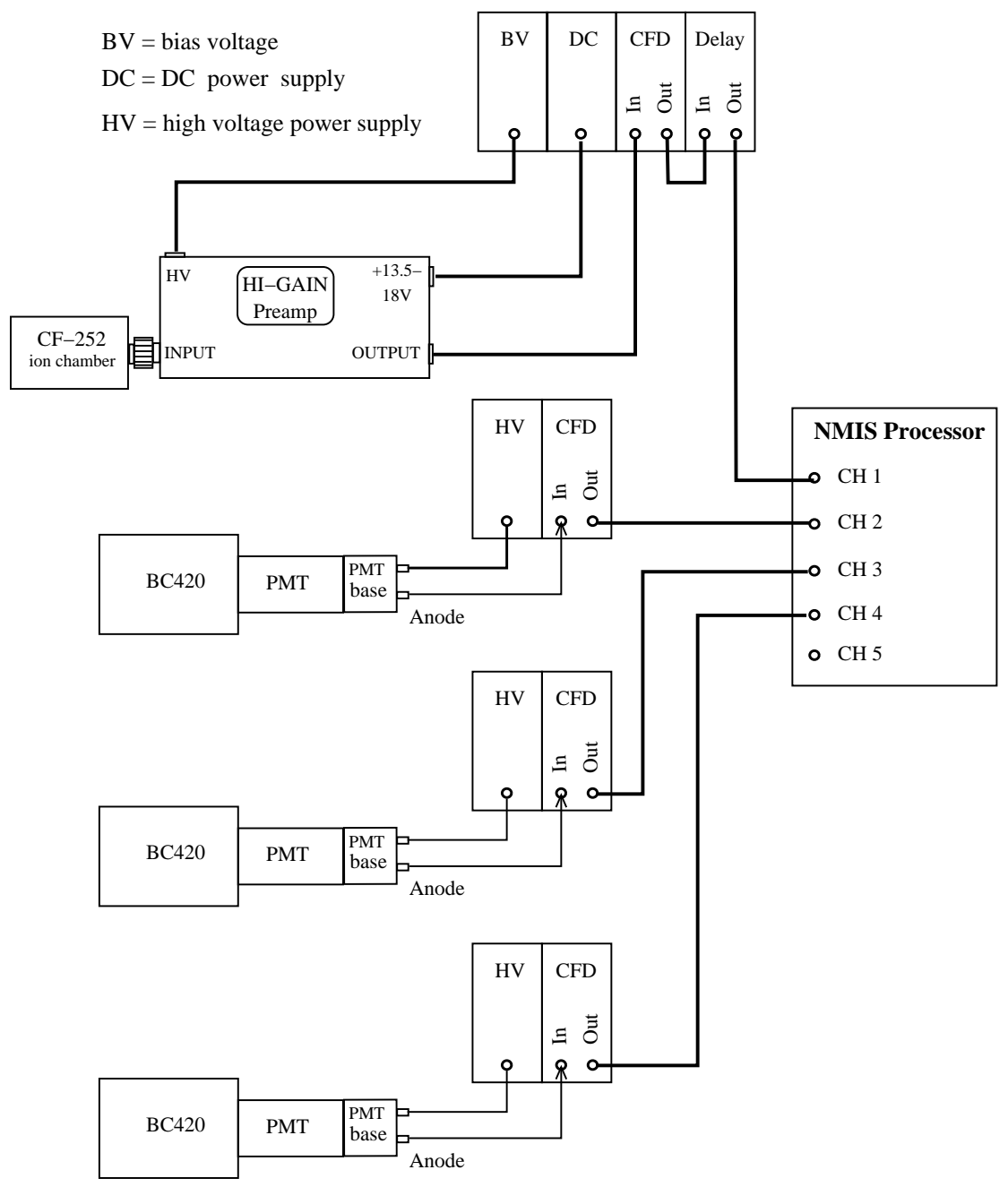

Figure 38: Block diagram of NMIS.

Table 9: Technical specifications for BC-420

\begin{tabular}{ll}
\hline material & polyvinyl toluene \\
density & $1.032 \mathrm{~g} / \mathrm{cm}^{3}$ \\
atomic ratio, $H / C$ & $\sim 1.1$ \\
atomic number density of hydrogen & $5.21 \times 10^{22} / \mathrm{cm}^{3}$ \\
atomic number density of carbon & $4.74 \times 10^{22} / \mathrm{cm}^{3}$ \\
electron density & $3.37 \times 10^{23} / \mathrm{cm}^{3}$ \\
light output, percent of anthracene & 64 \\
rise time & $0.5 \mathrm{~ns}$ \\
decay time & $1.5 \mathrm{~ns}$ \\
pulse width at FWHM & $1.3 \mathrm{~ns}$ \\
1/e light attenuation length & $140 \mathrm{~cm}$ \\
maximum wavelength of emission & $391 \mathrm{~nm}$ \\
\hline
\end{tabular}


set. The first experiment using the 3 -inch $(2.75 \times 2.75 \times 4 \text { in. })^{1} \quad$ detectors was performed on 2/15/01. The bias voltages on the photomultiplier tubes were $1470 \mathrm{~V}, 1365 \mathrm{~V}$, and $1435 \mathrm{~V}$ on detectors 1, 2, and 3 respectively. The threshold voltages on the detector CFDs were all set to $40 \mathrm{mV}$. This multiplicity data was acquired for 5000 loops which corresponds to 71 hours.

The second experiment used the 4 -inch detectors $(3.75 \times 3.75 \mathrm{x} 4 \mathrm{in}$.$) and was performed$ on 3/01/01. The multiplicity data for this experiment was also acquired for 5000 loops or 71 hours.

Table 10: Parameters for 4-inch detectors

\begin{tabular}{cccccc}
\hline $\begin{array}{c}\text { detector } \\
\text { number } \\
(\#)\end{array}$ & $\begin{array}{c}\text { serial } \\
\text { number }\end{array}$ & $\begin{array}{c}\text { bias } \\
\text { voltage } \\
(\mathrm{V})\end{array}$ & $\begin{array}{c}\text { neutron } \\
\text { threshold } \\
(\mathrm{MeV})\end{array}$ & $\begin{array}{c}\text { peak } \\
\text { efficiency } \\
(\%)\end{array}$ & $\begin{array}{c}\text { CFD } \\
\text { threshold } \\
(\mathrm{mV})\end{array}$ \\
\hline 2 & PH275 & 1550 & 0.96 & 60.0 & 40 \\
3 & PH276 & 1460 & 0.99 & 59.7 & 40 \\
4 & PH277 & 1470 & 0.96 & 59.0 & 40 \\
\hline
\end{tabular}

The geometry factor $\left(\frac{\Omega}{4 \pi}\right)$ for the 3 -inch detector is 0.00718 and 0.0131 for a 4 -inch detector.

\section{3 $\quad{ }^{252} C f$ ionization chamber source}

The source was developed by John Mihalczo and others.[23, 33] It consists of a $1 \mathrm{~cm}$ diameter spot of ${ }^{252} \mathrm{Cf}$ plated onto the center of a circular platinum disk. The disk is then oriented inside an ionization chamber in a parallel plate configuration as shown in Figure 39. The instrumented ${ }^{252} C f$ source is used to provide a trigger for NMIS whenever a source fission occurs. In order to detect when the fissions occur, a bias voltage is applied between the disk and the shell of the source container. The ionization chamber was filled with high purity $97 \% \mathrm{Ar}, 3 \% \mathrm{CO}_{2}$ gas mixture at 1 atm and operated in pulse-mode. Because the ionization chamber detects both the alpha decays and the spontaneous fissions from the

\footnotetext{
${ }^{1} 3$-inch and 4-inch is used throughout the thesis as the name of the detectors rather than the exact size. Both sets of detectors were 4 -inches thick.
} 
${ }^{252} C f$, the user must discriminate against the lower amplitude alpha pulses and electronic noise by properly setting the threshold voltage on the CFD. A CFD threshold setting of $80 \mathrm{mV}$ was used. Source CF-252-3412 SR\# 21 with an activity of $51.1 \mu \mathrm{Ci}$ on April 17, 2001 and a half life of 2.638 years was used in the experiments. Some pertinent data on the spontaneous decay of ${ }^{252} \mathrm{Cf}$ are summarized in Table 11.

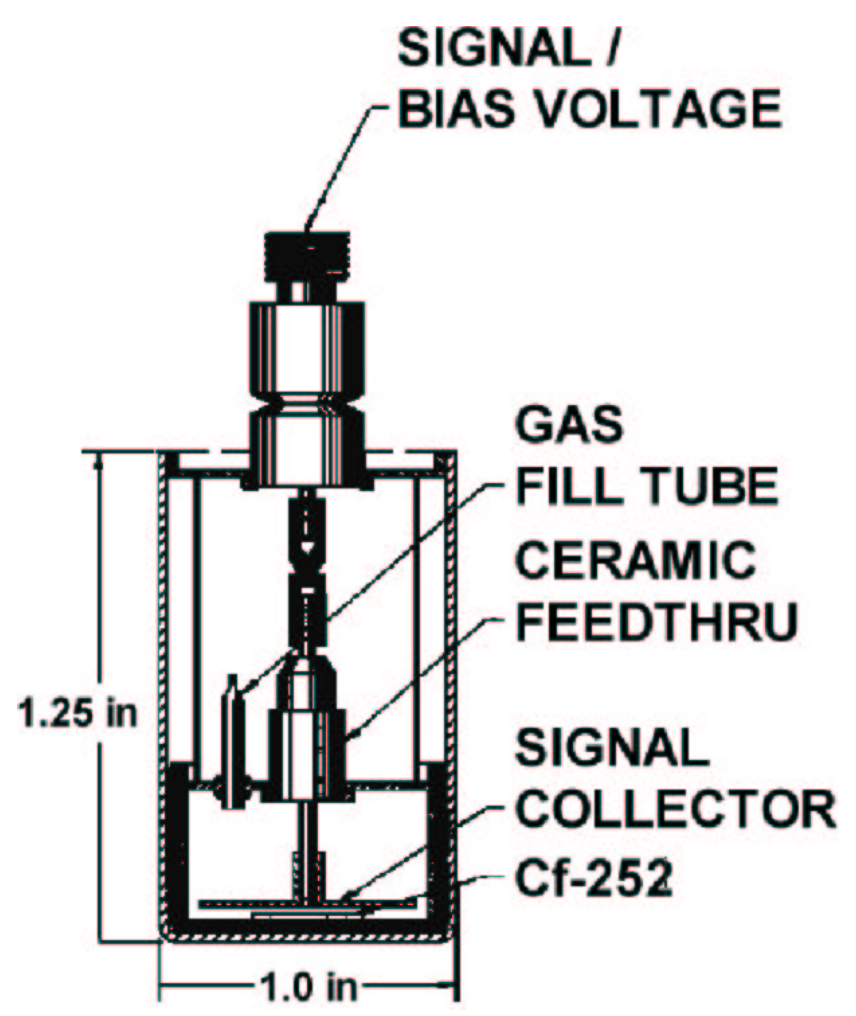

Figure 39: Schematic of ${ }^{252} C f$ ionization chamber.

Table 11: Spontaneous Decay of ${ }^{252} C f$

\begin{tabular}{ll}
\hline spontaneous fission specific activity & $6.14 \times 10^{5} / \mathrm{s} \cdot \mu \mathrm{g}$ \\
alpha-decay specific activity & $1.92 \times 10^{7} / \mathrm{s} \cdot \mu \mathrm{g}$ \\
energy of most probable light fission fragment & $\sim 105 \mathrm{MeV}$ \\
energy of most probable heavy fission fragment & $\sim 80 \mathrm{MeV}$ \\
average alpha energy & $6.11 \mathrm{MeV}$ \\
average energy of prompt neutron from spontaneous fission & $2.13 \mathrm{MeV}$ \\
average number of prompt neutrons from spontaneous fission & $3.77 /$ fission \\
\hline
\end{tabular}




\section{CHAPTER VIII}

\section{EXPERIMENTAL RESULTS}

This chapter presents the results of the experiments described in Chapter 7. The basic source-to-detector correlation $C_{1 j}\left(\tau_{1 j}\right)$ is displayed in Figure 40 for each of the three, 3 -inch detectors. The three detector-detector correlations $C_{i j}\left(\tau_{i j}\right)$ are shown in Figure 41. Because $d_{i}$ is $23 \mathrm{~cm}, \frac{d_{i}}{c}$ is $0.8 \mathrm{~ns}, \frac{d_{i}}{v_{f}}$ is about $5 \mathrm{~ns}, \frac{d_{i}}{v_{t}}$ is about $20 \mathrm{~ns}$ and $\Delta g$ is approximately $5 \mathrm{~ns}$. These parameters define the temporal boundaries as described in Section 4.1.

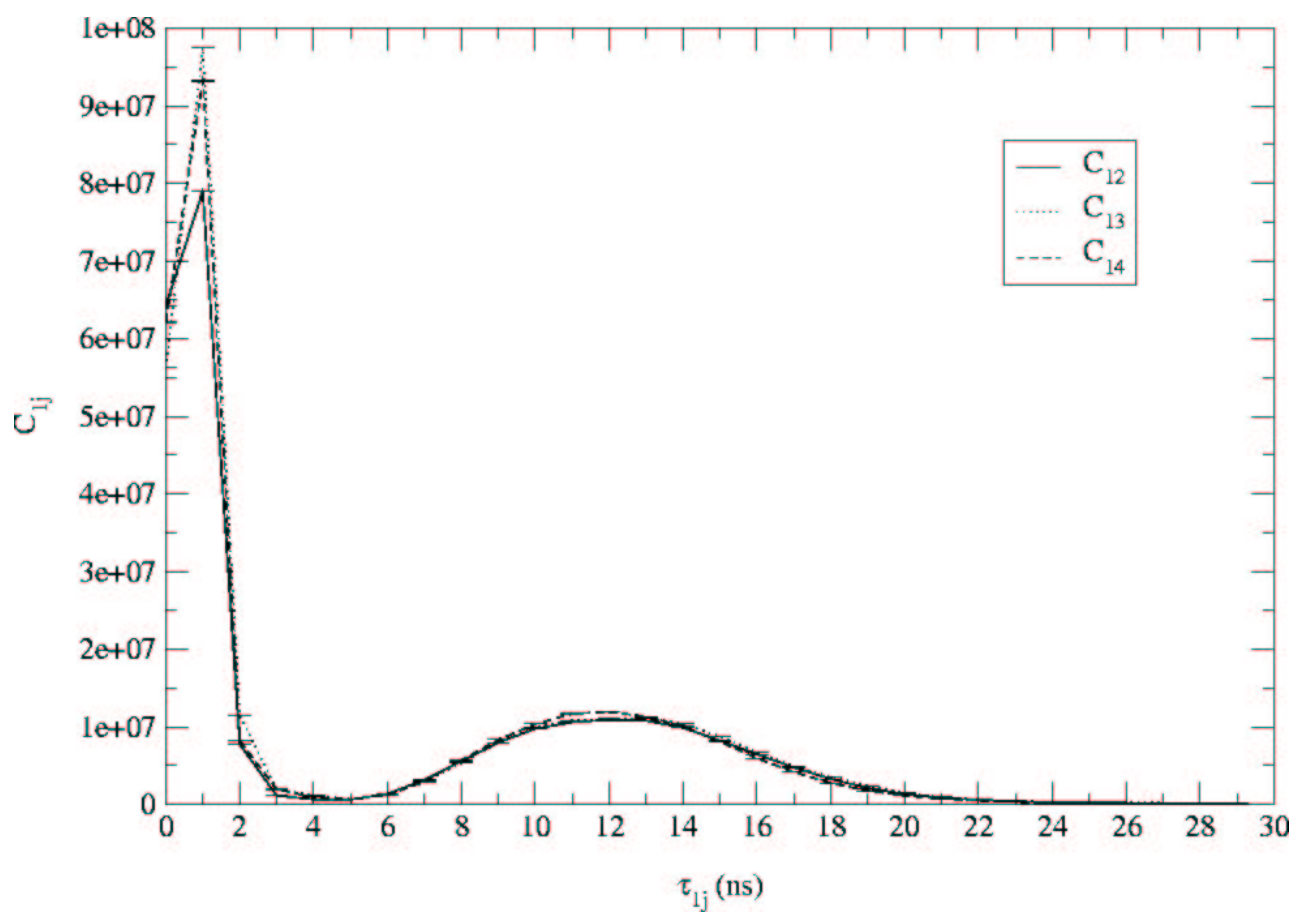

Figure 40: Experimental source-detector correlations $C_{1 j}\left(\tau_{1 j}\right)$ in total correlated counts for 3 -inch detectors.

The results of the auto-correlations $C_{i i}(0)$ are shown in Table $12 . C_{i i}(0)$ is the total number of detections in detector $i$ during the total counting time $T$. Dividing $C_{i i}(0)$ by this time gives the detection rate for the detector. The auto-correlation $C_{11}(0)$ represents the 


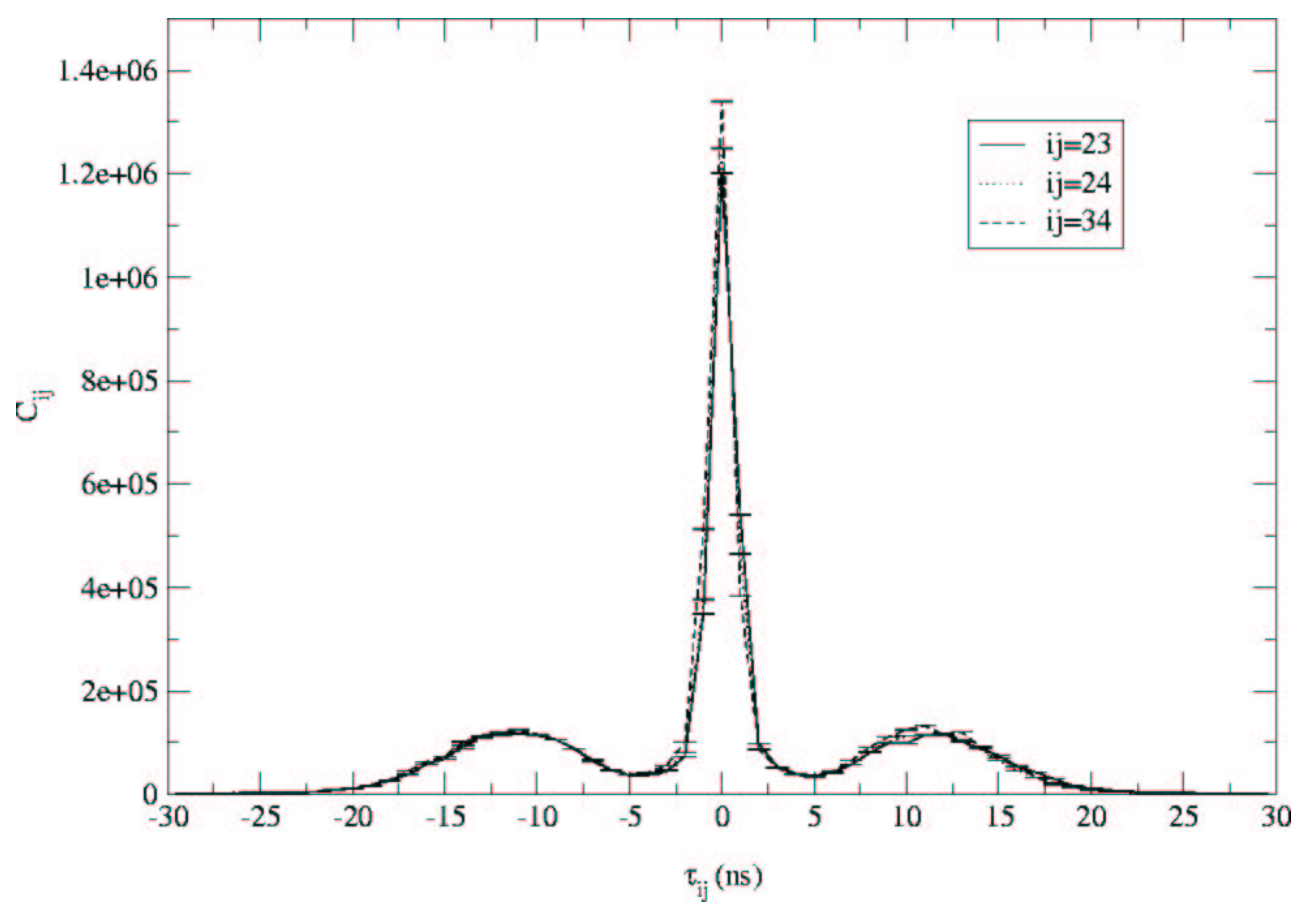

Figure 41: Experimental detector-detector correlations $C_{i j}\left(\tau_{i j}\right)$ in total correlated counts for 3 inch detectors.

total number of spontaneous fissions detected by the ${ }^{252} C f$ ion-chamber.

Table 12: Correlation data for 3 -inch and 4-inch detectors. $T=2.56 \times 10^{5} \mathrm{~s}$

\begin{tabular}{|r|rr|rr|}
\cline { 2 - 5 } \multicolumn{1}{c|}{} & \multicolumn{2}{|c|}{ 3-inch } & \multicolumn{2}{c|}{ 4-inch } \\
\hline $\mathrm{i}$ & \multicolumn{1}{c|}{$C_{i i}(0)$} & \multicolumn{1}{c|}{$r_{i}(0)$} & $r_{i}$ \\
\hline 1 & $18,148,280,000$ & $70891.71 s^{-1}$ & $17,936,460,000$ & $70064.29 s^{-1}$ \\
2 & $522,091,500$ & $2039.42 s^{-1}$ & $692,959,600$ & $2706.874 s^{-1}$ \\
3 & $561,954,600$ & $2195.135 s^{-1}$ & $664,763,600$ & $2596.733 s^{-1}$ \\
4 & $553,495,700$ & $2162.093 s^{-1}$ & $636,998,200$ & $2488.274 s^{-1}$ \\
\hline
\end{tabular}

First, source-detector measurements will be described. These measurements are not available in a passive measurement. However they provide useful information about the validity of the equations developed in Chapter 4 . In the next section the results of some of the passive detector ratios will be shown. 


\subsection{Source correlations}

In this section, the results of measurements of ratios developed in Section 4.2 are presented. Figure 42 shows the ratio $\frac{C_{i j \mid 1}\left(\tau_{i j}\right)}{C_{i j}\left(\tau_{i j}\right)}$ for the 3 -inch detectors. If spontaneous fission from the ${ }^{252} C f$ source were the only source measured, the ratio would be a constant. This constant would be the fission detection efficiency $\epsilon_{f}$ of the ${ }^{252} C f$ ion-chamber. The ratio is not constant, however. It is clearly a function of the delay variable $\tau_{i j}$. An explanation for this variation is correlated background.

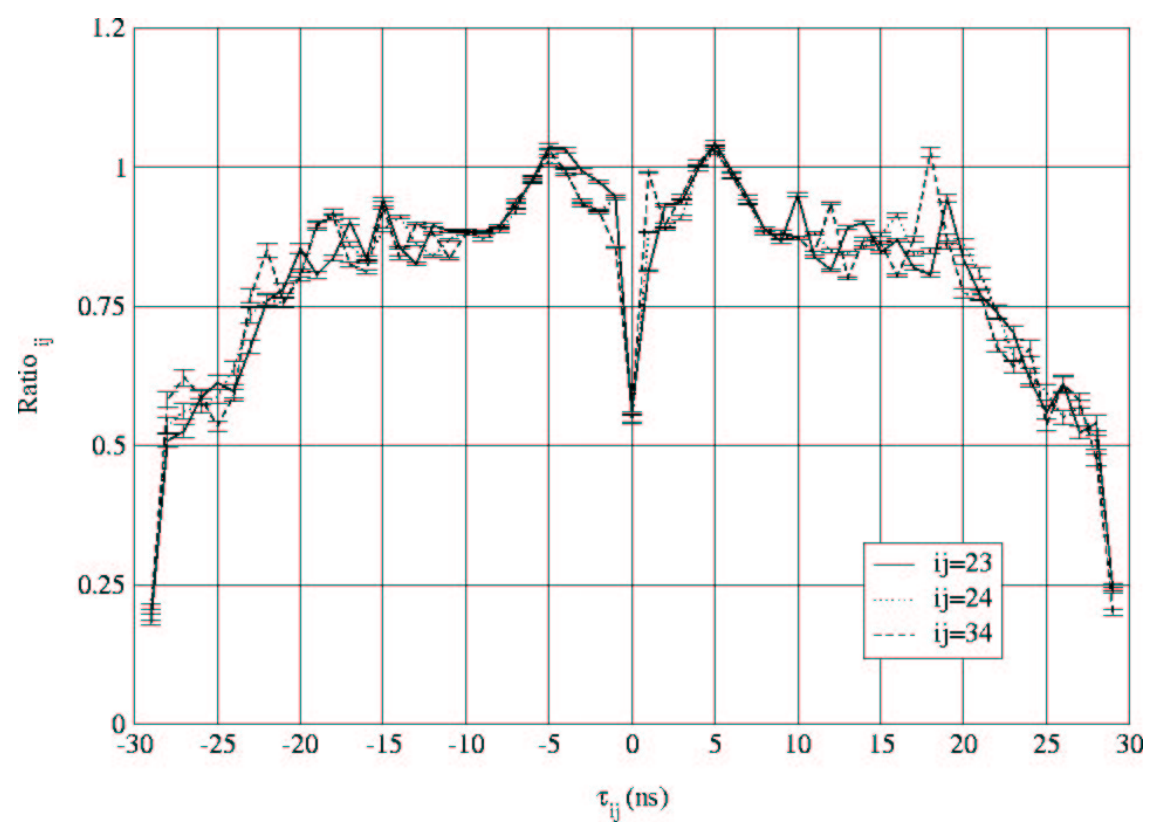

Figure 42: Ratio of $C_{i j \mid 1}\left(\tau_{i j}\right)$ with $C_{i j}\left(\tau_{i j}\right)$ from Equation (55).

First consider the $g g$ region around delay zero. There would be some $g g$ coincidences without the ${ }^{252} \mathrm{Cf}$ source present. This background could be measured. In addition, the ${ }^{252} \mathrm{Cf}$ source generates radioactive fission products. The radiations produced by these fission products are not correlated with the source fissions on the time scale of interest. Multiple gamma rays from the beta decay of these products will be correlated between detectors however. Other nuclear materials also produce radioactive nuclei through alpha decay as well. Consequently it is not a simple matter of measuring the background $g g$ pairs 
with the spontaneous fission source absent. In addition, $g g$ pairs are produced through inelastic scattering of neutrons and through $(\alpha, n)$ reactions. Recall that in Equation (58), gamma pairs from radioactive decay were included in the ratio. By also including gamma coincidences from inelastic scattering, the ratio becomes

$$
\frac{C_{i j \mid 1}}{C_{i j}}=\frac{\epsilon_{f} N_{1}\left(\overline{\gamma(\gamma-1)}+p_{i} \overline{\gamma_{i}\left(\gamma_{i}-1\right)}\right)}{\left(N_{1} \overline{\gamma(\gamma-1)}+N_{r} \overline{\gamma_{r}\left(\gamma_{r}-1\right)}+N_{i} \overline{\gamma_{i}\left(\gamma_{i}-1\right)}\right)}
$$

where $p_{i}$ is the probability of an inelastic scatter from fission neutrons, and in addition to $N_{r}$, the number of radioactive decays, $N_{i}$ is the total number of inelastic scatters from all neutrons in the vicinity of the detectors. The numerator represents gamma detections which are correlated with source fissions. They include fission gamma rays as well as gamma rays from the inelastic scattering of fission neutrons. The numerator represents detectordetector correlations not necessarily correlated with the source. These correlations include those from fission gamma rays, gamma rays from radioactive decay and inelastic scattering.

The ratio in Figure 42 peaks at about 5 ns. Although $n n$ pairs should be visible in this region, the alternative possibility of gamma scattering exists and could result in values larger than unity. Some evidence of this scattering can be seen in Figure 11.

Assuming that the $g n$ and $n g$ regions of Figure 42 provide the most reliable measure of fission detection efficiency $\epsilon_{f}$, the fission detection efficiency can be estimated to be about $90 \% \pm 10 \%$. From Table 12, the number of detected fissions is $1.81 \times 10^{10}$ and $1.79 \times 10^{10}$ respectively for the 3 -inch and 4-inch experiments. The number of actual fissions is then $2.01 \times 10^{10}$ and $1.99 \times 10^{10}$ which implies a ${ }^{252} C f$ mass of 0.128 and $0.127 \mu$ g respectively.

Another ratio involving source correlations in Equation (126) is shown in Figure 43. The value of this ratio in the gamma-neutron regions is one. Using the data from Tables 5 and 6 , the value in the $g g$ region is 0.98 and 1.18 in the $n n$ region. The graph undershoots in the $g g$ region indicating that the multiplicity distribution for gamma rays from Section 4.4.2 is not correct for the gamma rays detected. The value in the $n n$ region appears to be about 


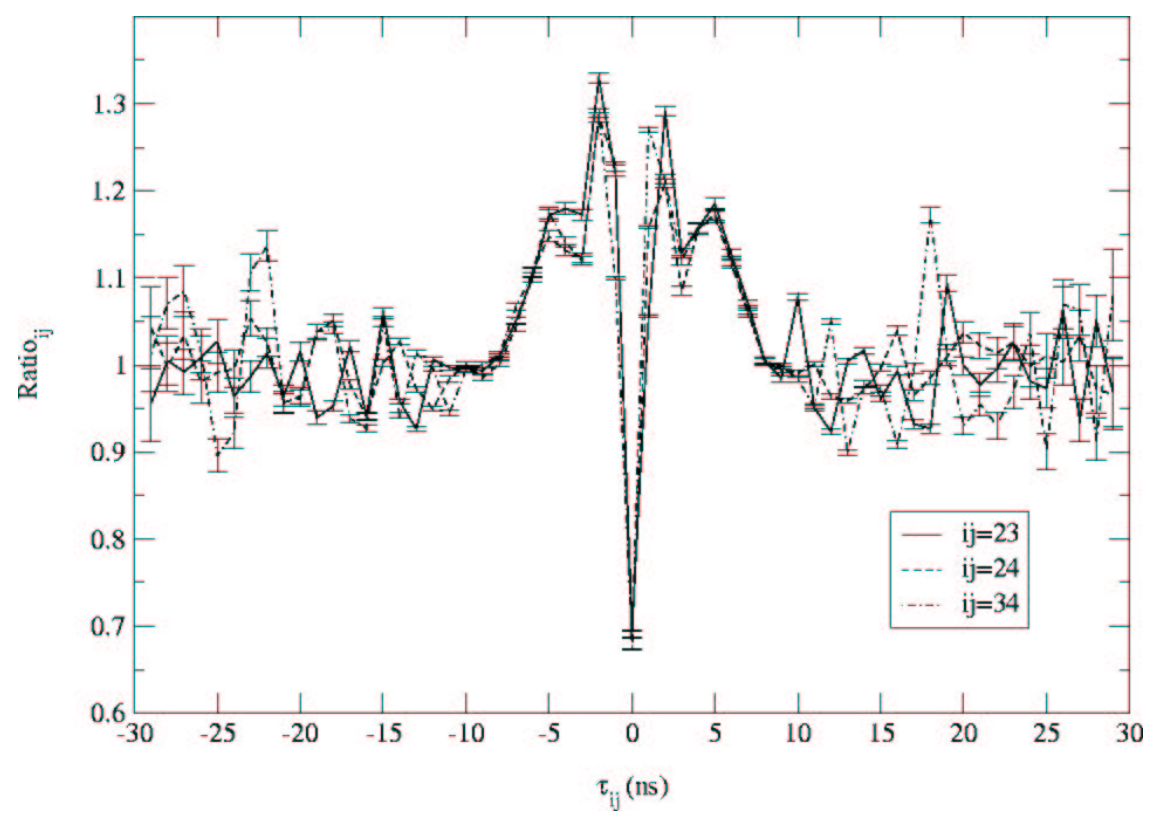

Figure 43: Ratio of $\int_{0}^{\infty} C_{1 i}\left(\tau_{1 i}\right) C_{1 j}\left(\tau_{i j}+\tau_{1 i}\right) d \tau_{1 i}$ with $\int_{0}^{\infty} C_{1 i j}\left(\tau_{1 i}, \tau_{i j}+\tau_{1 i}\right) d \tau_{1 i}$ from Equation (60).

right except for an overshoot just adjacent to the $g g$ region.

$$
\frac{\int_{-\infty}^{\infty} C_{1 i}\left(\tau_{1 i}\right) C_{1 j}\left(\tau_{i j}+\tau_{1 i}\right) d \tau_{1 i}}{\left.N_{1} \epsilon_{f} \int_{-\infty}^{\infty} C_{123}\left(\tau_{12}, \tau_{i j}+\tau_{1 i}\right)\right) d \tau_{1 i}}=\underbrace{\frac{\bar{\gamma}^{2}}{\overline{\gamma(\gamma-1)}}}_{\mathrm{gg}}+\underbrace{\frac{\bar{\nu}^{2}}{\overline{\nu(\nu-1)}}}_{\mathrm{nn}}+\underbrace{1}_{\mathrm{ng} \text { or gn }}
$$

\section{$\underline{8.2}$ Passive detector correlations}

Ratios of passive detector-detector correlations were developed in Section 4.3. The ratios with all time variables integrated out will first be presented, and detector efficiency and the mass of ${ }^{252} C f$ will be calculated from these ratios. Then in the subsequent Section 8.2.1, the time dependent ratios will be presented. A comparison between the results from the 3 -inch and 4-inch detectors will be presented in Section 8.3 . Finally ${ }^{252} C f$ mass will also be calculated from gnn coincidences in Section 8.4.

The results of the passive measurements of Equations (127) to (132) are shown in Table 13 for the 3-inch detectors and Table 14 for the 4-inch detectors. Evaluation of the factorial moments comes from Section 4.4.1 for neutrons and Section 4.4.2 for gamma rays. These factorial moments are for a non-multiplying system $(M=1)$. In the case of a 
multiplying system, these moments are replaced with those derived in Chapter 5 in which case $M$ becomes an additional unknown. From these measurements, the neutron detection efficiency can be computed from Equation (130) or from Equation (128) with less reliable results. The gamma ray detection efficiency can be computed from Equation (130) or Equation (127) with less reliable results. Recall from Section 8.1 that there are many sources of all-gamma combinations which make Equations (127) and (128) troublesome. There are three measurements for each of these equations, one for each of the pairs of detectors $(23$, 24 and 34). The results for these detection efficiencies are shown in Tables 15 and 16 .

$$
\begin{gathered}
\frac{C_{g g}}{C_{g g g}}=\frac{\overline{\gamma(\gamma-1)}}{\overline{\gamma(\gamma-1)(\gamma-2) \epsilon_{g}}}=\frac{0.11}{\epsilon_{g}} \\
\frac{C_{g g \mid n}}{C_{g g}}=\bar{\nu} \epsilon_{n}=3.77 \epsilon_{n} \\
\frac{C_{g n \mid g}}{C_{g n}}=\frac{\overline{\gamma(\gamma-1)}}{\bar{\gamma}} \epsilon_{g}=8.54 \epsilon_{g} \\
\frac{C_{g n \mid n}}{C_{g n}}=\frac{\frac{\nu(\nu-1)}{\bar{\nu}}}{\epsilon_{n}}=3.19 \epsilon_{n}
\end{gathered}
$$

The following two equations (131) and (132) can be used to determine the number of fissions $N$. The mass of ${ }^{252} C f$ is then determined from the specific activity and total measurement time $T$ by $m_{c f}=\frac{N}{6.14 \times 10^{5} / s \cdot \mu g \cdot T}$.

$$
\begin{aligned}
& \frac{\left(C_{g n}\right)^{2}}{C_{g n \mid g}}=N \frac{\bar{\gamma}^{2} \bar{\nu}}{\overline{\gamma(\gamma-1)}} \epsilon_{n}=3.53 N \epsilon_{n} \\
& \frac{\left(C_{g n}\right)^{2}}{C_{g n \mid n}}=N \frac{\bar{\nu}^{2} \bar{\gamma}}{\overline{\nu(\nu-1)}} \epsilon_{g}=9.43 N \epsilon_{g}
\end{aligned}
$$

With the detection efficiencies $\epsilon_{g}$ and $\epsilon_{n}$ already determined, the number of spontaneous fissions $N$ are easily calculated from Equations (131) and (132). The results of these calculations are shown in Table 17. 
Table 13: Passive ratios for 3 -inch detectors

\begin{tabular}{|c|c|c|c|c|c|c|c|}
\hline Equation & Combination & $\tau_{23}$ & $\frac{\sigma}{R}(\%)$ & $\tau_{24}$ & $\frac{\sigma}{R}(\%)$ & $\tau_{34}$ & $\frac{\sigma}{R}(\%)$ \\
\hline 127 & $g g g$ & 107.0 & 0.7 & 106.9 & 0.7 & 114.8 & 0.7 \\
\hline \multirow{3}{*}{128} & $g g n$ & 0.00457 & 1.0 & - & - & - & - \\
\hline & $g n g$ & - & - & 0.00466 & 1.0 & - & - \\
\hline & $n g g$ & - & - & - & - & 0.00446 & 1.0 \\
\hline \multirow{3}{*}{129} & $g n g$ & 0.00933 & 1.0 & - & - & 0.00864 & 1.0 \\
\hline & $g g n$ & - & - & 0.00945 & 1.0 & 0.00869 & 1.0 \\
\hline & $n g g$ & 0.00918 & 1.0 & 0.00917 & 1.0 & - & - \\
\hline \multirow{3}{*}{130} & $g n n$ & 0.00445 & 1.5 & 0.00459 & 1.5 & - & - \\
\hline & $n g n$ & 0.00448 & 1.4 & - & - & 0.00444 & 1.4 \\
\hline & $n n g$ & - & - & 0.00434 & 1.4 & 0.00433 & 1.4 \\
\hline \multirow{3}{*}{131} & $g n g$ & $1.21 \mathrm{E}+08$ & 1.0 & - & - & $1.41 \mathrm{E}+08$ & 1.0 \\
\hline & $g g n$ & - & - & $1.16 \mathrm{E}+08$ & 1.0 & $1.37 \mathrm{E}+08$ & 1.0 \\
\hline & $n g g$ & $1.28 \mathrm{E}+08$ & 1.0 & $1.29 \mathrm{E}+08$ & 1.0 & - & - \\
\hline \multirow{3}{*}{132} & $g n n$ & $2.54 \mathrm{E}+08$ & 1.4 & $2.38 \mathrm{E}+08$ & 1.4 & - & - \\
\hline & $n g n$ & $2.63 \mathrm{E}+08$ & 1.4 & - & - & $2.68 \mathrm{E}+08$ & 1.4 \\
\hline & $n n g$ & - & - & $2.72 \mathrm{E}+08$ & 1.4 & $2.82 \mathrm{E}+08$ & 1.4 \\
\hline
\end{tabular}

Table 14: Passive ratios for 4-inch detectors

\begin{tabular}{|c|c|c|c|c|c|c|c|}
\hline Equation & Combination & $\tau_{23}$ & $\frac{\sigma}{R}(\%)$ & $\tau_{24}$ & $\frac{\sigma}{R}(\%)$ & $\tau_{34}$ & $\frac{\sigma}{R}(\%)$ \\
\hline 127 & $g g g$ & 118.6 & 0.6 & 115.6 & 0.6 & 109.9 & 0.6 \\
\hline \multirow{3}{*}{128} & $g g n$ & 0.00734 & 0.6 & - & - & - & - \\
\hline & $g n g$ & - & - & 0.00790 & 0.6 & - & - \\
\hline & $n g g$ & - & - & - & - & 0.00777 & 0.7 \\
\hline \multirow{3}{*}{129} & $g n g$ & 0.00905 & 0.6 & - & - & 0.00976 & 0.6 \\
\hline & $g g n$ & - & - & 0.00926 & 0.7 & 0.00977 & 0.6 \\
\hline & $n g g$ & 0.00885 & 0.7 & 0.00906 & 0.7 & - & \\
\hline \multirow{3}{*}{130} & $g n n$ & 0.00762 & 0.7 & 0.00817 & 0.7 & - & - \\
\hline & $n g n$ & 0.00764 & 0.7 & - & - & 0.00827 & 0.7 \\
\hline & $n n g$ & - & - & 0.00800 & 0.7 & 0.00788 & 0.7 \\
\hline \multirow{3}{*}{131} & $g n g$ & $3.13 \mathrm{E}+08$ & 0.6 & - & - & $2.69 \mathrm{E}+08$ & 0.6 \\
\hline & $g g n$ & - & - & $2.85 \mathrm{E}+08$ & 0.6 & $2.57 \mathrm{E}+08$ & 0.6 \\
\hline & $n g g$ & $3.06 \mathrm{E}+08$ & 0.6 & $2.92 \mathrm{E}+08$ & 0.6 & - & - \\
\hline \multirow{3}{*}{132} & $g n n$ & $3.72 \mathrm{E}+08$ & 0.7 & $3.23 \mathrm{E}+08$ & 0.7 & - & - \\
\hline & $n g n$ & $3.55 \mathrm{E}+08$ & 0.7 & - & - & $3.03 \mathrm{E}+08$ & 0.7 \\
\hline & $n n g$ & - & - & $3.31 \mathrm{E}+08$ & 0.7 & $3.33 \mathrm{E}+08$ & 0.7 \\
\hline
\end{tabular}


Table 15: Gamma-ray detection efficiency for detector $k$. Average gamma-ray detection efficiency for 3 -inch detectors is $\epsilon_{g}=1.04 \times 10^{-03} \pm 4.5 \%$ and 4-inch detectors is $\epsilon_{g}=$ $1.04 \times 10^{-03} \pm 7.3 \%$.

\begin{tabular}{lccccccc}
\hline Equation & Combination & \multicolumn{3}{c}{ Index } & \multicolumn{2}{c}{3 -inch } & \multicolumn{2}{c}{ 4-inch } \\
Number & & ij & $k$ & $\epsilon_{g k}$ & $\frac{\sigma}{\epsilon}(\%)$ & $\epsilon_{g k}$ & $\frac{\sigma}{\epsilon}(\%)$ \\
\hline \multirow{3}{*}{127} & $g g g$ & 23 & 4 & $1.03 \mathrm{E}-03$ & 0.7 & $9.26 \mathrm{E}-04$ & 0.6 \\
& $g g g$ & 24 & 3 & $1.03 \mathrm{E}-03$ & 0.7 & $9.51 \mathrm{E}-04$ & 0.6 \\
& $g g g$ & 34 & 2 & $9.58 \mathrm{E}-04$ & 0.7 & $1.00 \mathrm{E}-03$ & 0.6 \\
\hline \multirow{4}{*}{129} & $g n g$ & 23 & 4 & $1.09 \mathrm{E}-03$ & 1.0 & $1.06 \mathrm{E}-03$ & 0.6 \\
& $n g g$ & 23 & 4 & $1.07 \mathrm{E}-03$ & 1.0 & $1.04 \mathrm{E}-03$ & 0.7 \\
& $g g n$ & 24 & 3 & $1.11 \mathrm{E}-03$ & 1.0 & $1.08 \mathrm{E}-03$ & 0.7 \\
& $n g g$ & 24 & 3 & $1.07 \mathrm{E}-03$ & 1.0 & $1.06 \mathrm{E}-03$ & 0.7 \\
& $g g n$ & 34 & 2 & $1.02 \mathrm{E}-03$ & 1.0 & $1.14 \mathrm{E}-03$ & 0.6 \\
& $g n g$ & 34 & 2 & $1.01 \mathrm{E}-03$ & 1.0 & $1.14 \mathrm{E}-03$ & 0.6 \\
\hline
\end{tabular}

Table 16: Neutron detection efficiency for detector $k$. Average neutron detection efficiency for 3-inch detectors is $\epsilon_{n}=$ $1.33 \times 10^{-03} \pm 7.1 \%$ and 4-inch is $\epsilon_{n}=2.33 \times 10^{-03} \pm 10.2 \%$.

\begin{tabular}{lccccccc}
\hline Equation & Combination & \multicolumn{2}{c}{ Index } & \multicolumn{2}{c}{3 -inch } & \multicolumn{2}{c}{ 4-inch } \\
Number & & ij & $k$ & $\epsilon_{n k}$ & $\frac{\sigma}{\epsilon}(\%)$ & $\epsilon_{n k}$ & $\frac{\sigma}{\epsilon}(\%)$ \\
\hline \multirow{2}{*}{128} & $g g n$ & 23 & 4 & $1.21 \mathrm{E}-03$ & 1.0 & $1.95 \mathrm{E}-03$ & 0.6 \\
& $g n g$ & 24 & 3 & $1.24 \mathrm{E}-03$ & 1.0 & $2.09 \mathrm{E}-03$ & 0.6 \\
& $n g g$ & 34 & 2 & $1.18 \mathrm{E}-03$ & 1.0 & $2.06 \mathrm{E}-03$ & 0.7 \\
\hline \multirow{4}{*}{130} & $g n n$ & 23 & 4 & $1.39 \mathrm{E}-03$ & 1.5 & $2.39 \mathrm{E}-03$ & 0.7 \\
& $n g n$ & 23 & 4 & $1.40 \mathrm{E}-03$ & 1.4 & $2.39 \mathrm{E}-03$ & 0.7 \\
& $g n n$ & 24 & 3 & $1.44 \mathrm{E}-03$ & 1.5 & $2.56 \mathrm{E}-03$ & 0.7 \\
& $n n g$ & 24 & 3 & $1.36 \mathrm{E}-03$ & 1.4 & $2.51 \mathrm{E}-03$ & 0.7 \\
& $n g n$ & 34 & 2 & $1.39 \mathrm{E}-03$ & 1.4 & $2.59 \mathrm{E}-03$ & 0.7 \\
& $n n g$ & 34 & 2 & $1.36 \mathrm{E}-03$ & 1.4 & $2.47 \mathrm{E}-03$ & 0.7 \\
\hline
\end{tabular}


Table 17: Ratio of $\left(C_{g n}\right)^{2}$ with $C_{g n \mid g}$ and $C_{g n \mid n}$ for 3 -inch detectors $\left(N=2.71 \times 10^{10} \pm 6.5 \%, m_{c f}=0.18 \mu g\right)$ and 4 inch $\left(N=3.45 \times 10^{10} \pm 7.1 \%, m_{c f}=0.22 \mu g\right)$ detectors.

\begin{tabular}{lcccc}
\hline $\begin{array}{c}\text { Equation } \\
\text { Number }\end{array}$ & Combination & $\begin{array}{c}\text { Index } \\
i j\end{array}$ & $\begin{array}{c}3 \text {-inch } \\
N\end{array}$ & $\begin{array}{c}4 \text {-inch } \\
N\end{array}$ \\
\hline \multirow{4}{*}{131} & & 23 & $2.58 \mathrm{E}+10$ & $3.81 \mathrm{E}+10$ \\
& $n g g$ & 23 & $2.74 \mathrm{E}+10$ & $3.72 \mathrm{E}+10$ \\
& $g g n$ & 24 & $2.47 \mathrm{E}+10$ & $3.47 \mathrm{E}+10$ \\
& $n g g$ & 24 & $2.74 \mathrm{E}+10$ & $3.55 \mathrm{E}+10$ \\
& $g g n$ & 34 & $2.92 \mathrm{E}+10$ & $3.12 \mathrm{E}+10$ \\
& $g n g$ & 34 & $3.01 \mathrm{E}+10$ & $3.27 \mathrm{E}+10$ \\
\hline \multirow{4}{*}{32} & $g n n$ & 23 & $2.58 \mathrm{E}+10$ & $3.78 \mathrm{E}+10$ \\
& $n g n$ & 23 & $2.67 \mathrm{E}+10$ & $3.60 \mathrm{E}+10$ \\
& $g n n$ & 24 & $2.42 \mathrm{E}+10$ & $3.28 \mathrm{E}+10$ \\
& $n n g$ & 24 & $2.76 \mathrm{E}+10$ & $3.35 \mathrm{E}+10$ \\
& $n g n$ & 34 & $2.72 \mathrm{E}+10$ & $3.07 \mathrm{E}+10$ \\
& $n n g$ & 34 & $2.86 \mathrm{E}+10$ & $3.38 \mathrm{E}+10$ \\
\hline
\end{tabular}

The results for the number of fissions $N$ and ${ }^{252} C f$ mass shown in Table 17 have an unfortunate disagreement between the 3 -inch and 4 -inch detectors. The results of the experiment with the 3 -inch detectors estimates the mass of ${ }^{252} C f$ to be $0.18 \mu \mathrm{g}$. The result for the 4-inch detectors is $0.22 \mu \mathrm{g}$. The problem can be attributed to contamination of the gamma-neutron combinations with neutron triples. A comparison of the 3-inch and 4-inch detector data will be examined following an examination of the time dependence of the passive ratios used.

\subsubsection{Time dependent ratios}

Equations (127) to (132) can also be written with one of the time variables $\tau_{i j}$ left in. In this case, the final integration is not performed. These equations are shown below.

$$
\begin{gathered}
\frac{C_{g n \mid g}\left(\tau_{i j}\right)}{C_{g n}\left(\tau_{i j}\right)}=\frac{\overline{\gamma(\gamma-1)}}{\bar{\gamma}} \epsilon_{g} \\
\frac{C_{g n \mid n}\left(\tau_{i j}\right)}{C_{g n}\left(\tau_{i j}\right)}=\frac{\overline{\nu(\nu-1)}}{\bar{\nu}} \epsilon_{n}\left(\tau_{i j}\right)
\end{gathered}
$$




$$
\begin{gathered}
\frac{\left(C_{g n}\left(\tau_{i j}\right)\right)^{2}}{C_{g n \mid g}\left(\tau_{i j}\right)}=N \frac{\bar{\gamma}^{2} \bar{\nu}}{\overline{\gamma(\gamma-1)}} \epsilon_{n}\left(\tau_{i j}\right) \\
\frac{\left(C_{g n}\left(\tau_{i j}\right)\right)^{2}}{C_{g n \mid n}\left(\tau_{i j}\right)}=N \frac{\bar{\nu}^{2} \bar{\gamma}}{\overline{\nu(\nu-1)}} \epsilon_{g}
\end{gathered}
$$

Equations (133) and (134) are the time dependent version of the equations used to determine the detection efficiency for gamma rays and neutrons respectively. Each equation can be integrated with respect to any of the three time variables, $\tau_{23}, \tau_{24}$ and $\tau_{34}$. Each of these integrations is on one of the three regions of $g g n$ and $g n n$. Graphs of these time dependent ratios for the 3 -inch detectors are shown in Figure 44 for $\tau_{23}$ dependence, Figure 45 for $\tau_{24}$ dependence and Figure 46 for $\tau_{34}$ dependence. These graphs show a region of time independence. The ratio with $\tau_{34}$ dependence shows considerable distortion. This distortion is not surprising since $C_{234}\left(\tau_{23}, \tau_{24}\right)$ is not a function of $\tau_{34}$. The integration is nevertheless performed with a substitution of variables based on the relation, $\tau_{34}=\tau_{24}-\tau_{23}$. However, the fully integrated ratio from Table 17 shows that this region is consistent with the other two regions.

Equations (135) and (136) are the time dependent version of the equations used to determine the total number of fissions $N$. Viewing the time dependence is useful in diagnosing why the results were less than satisfactory. Graphs of these rations are shown in Figures 47, 48 and 49 . The effect of $n n$ and $n n n$ contamination is evident in the graphs. This influence can be seen in the region between \pm 10 ns. The minimum in that region is more than an order of magnitude greater than at the long delays.

\subsection{Comparison between 3-inch and 4-inch detectors}

The results in the experiments between the 3-inch and 4-inch detectors have some inconsistencies. The 4-inch detector resulted in a higher ${ }^{252} C f$ mass determination from Equations 131 and 132.

The first anomaly between the 3-inch and 4-inch detectors is that the gamma-ray detection efficiency from Table 15 between the two are nearly identical. From the solid angle 


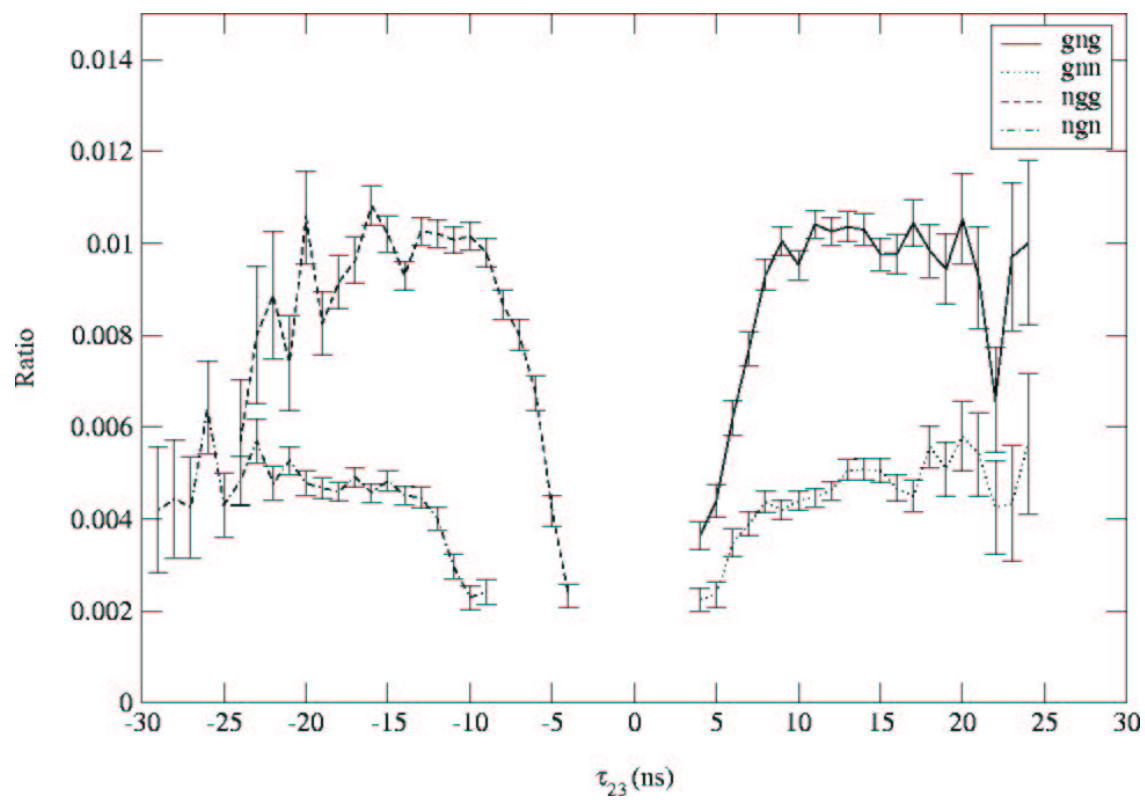

Figure 44: Ratio of $C_{n g \mid n}\left(\tau_{23}\right)$ and $C_{n g \mid g}\left(\tau_{23}\right)$ with $C_{n g}\left(\tau_{23}\right)$ on the left and ratio of $C_{g n \mid n}\left(\tau_{23}\right)$ and $C_{g n \mid g}\left(\tau_{23}\right)$ with $C_{g n}\left(\tau_{23}\right)$ on the right from Equations (133) and (134).

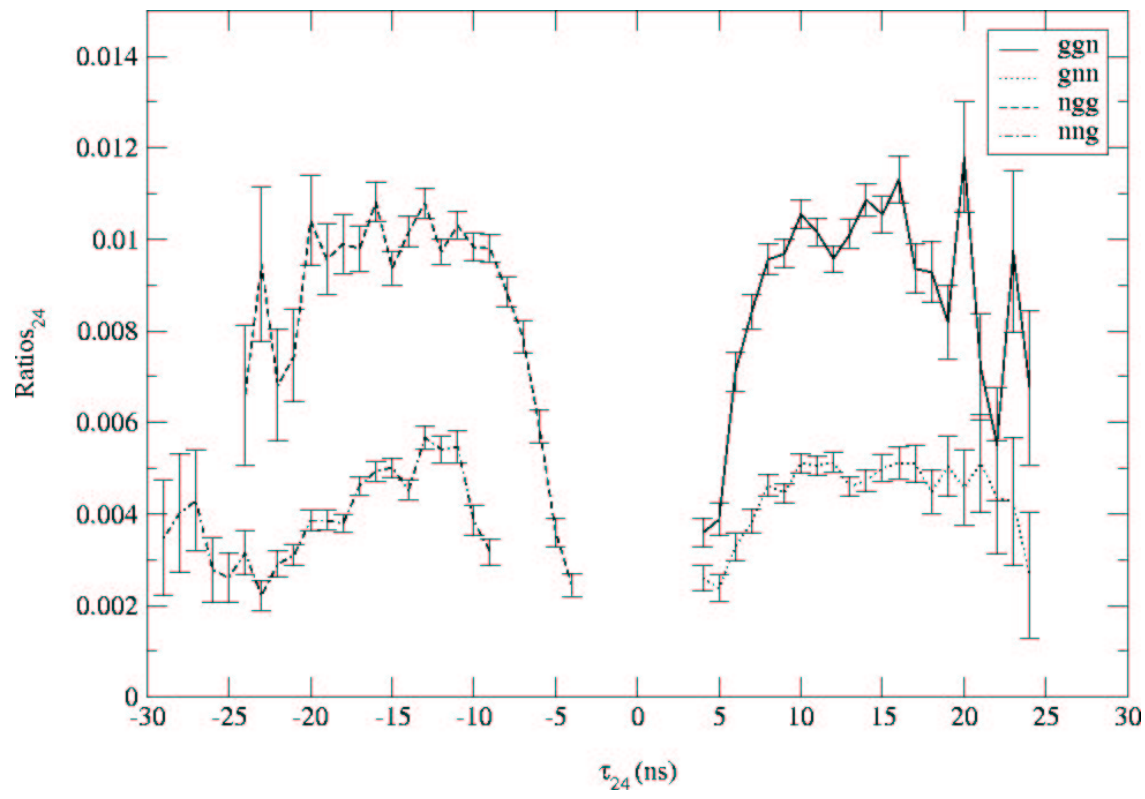

Figure 45: Ratio of $C_{n g \mid n}\left(\tau_{24}\right)$ and $C_{n g \mid g}\left(\tau_{24}\right)$ with $C_{n g}\left(\tau_{24}\right)$ on the left and ratio of $C_{g n \mid n}\left(\tau_{24}\right)$ and $C_{g n \mid g}\left(\tau_{24}\right)$ with $C_{g n}\left(\tau_{24}\right)$ on the right from Equations (133) and (134). 


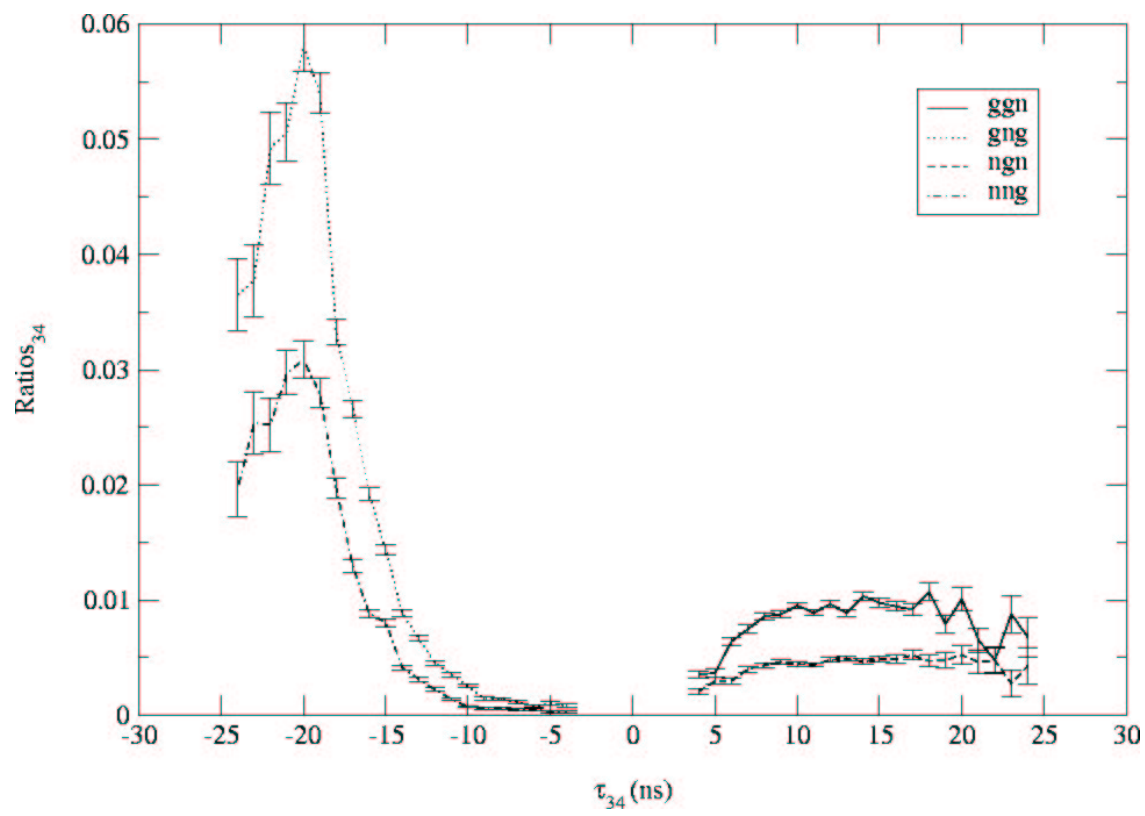

Figure 46: Ratio of $C_{n g \mid n}\left(\tau_{34}\right)$ and $C_{n g \mid g}\left(\tau_{34}\right)$ with $C_{n g}\left(\tau_{34}\right)$ on the left and ratio of $C_{g n \mid n}\left(\tau_{34}\right)$ and $C_{g n \mid g}\left(\tau_{34}\right)$ with $C_{g n}\left(\tau_{34}\right)$ on the right from Equations (133) and (134).

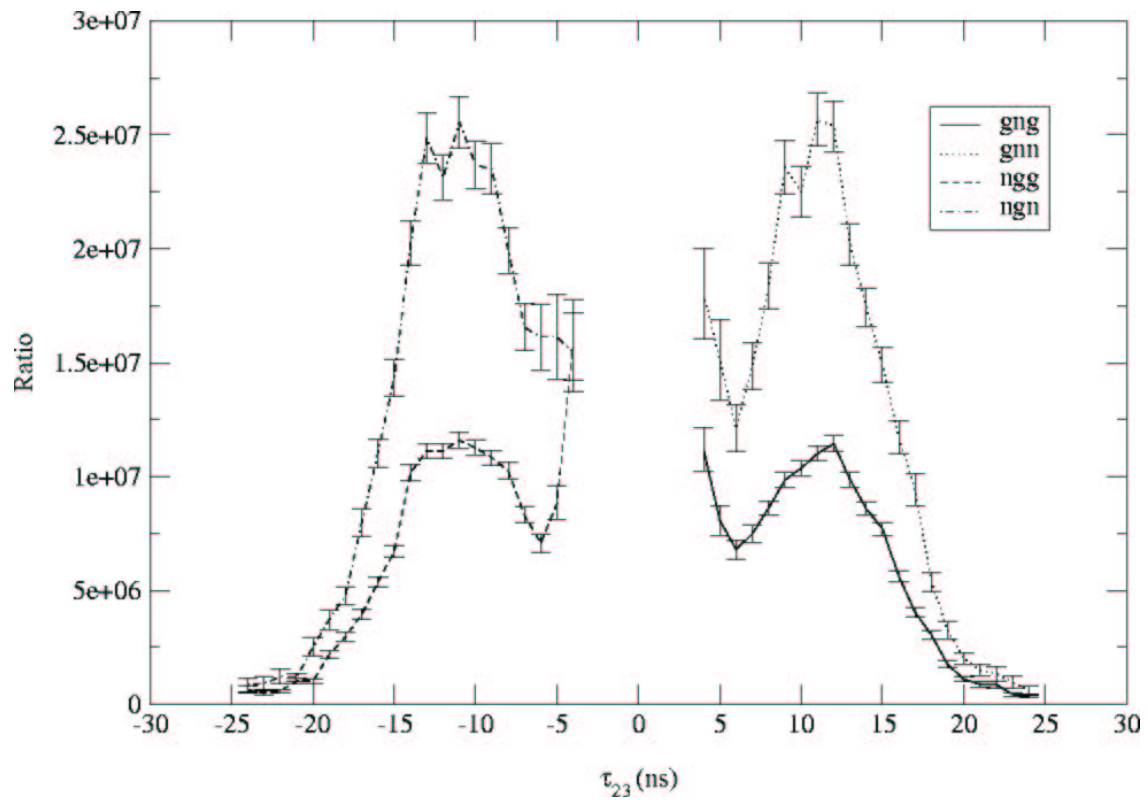

Figure 47: Ratio of $C_{n g}\left(\tau_{23}\right)^{2}$ with $C_{n g \mid n}\left(\tau_{23}\right)$ and $C_{n g \mid g}\left(\tau_{23}\right)$ on the left and ratio of $C_{g n}\left(\tau_{23}\right)^{2}$ with $C_{g n \mid n}\left(\tau_{23}\right)$ and $C_{g n \mid g}\left(\tau_{23}\right)$ on the right from Equations (135) and (136). 


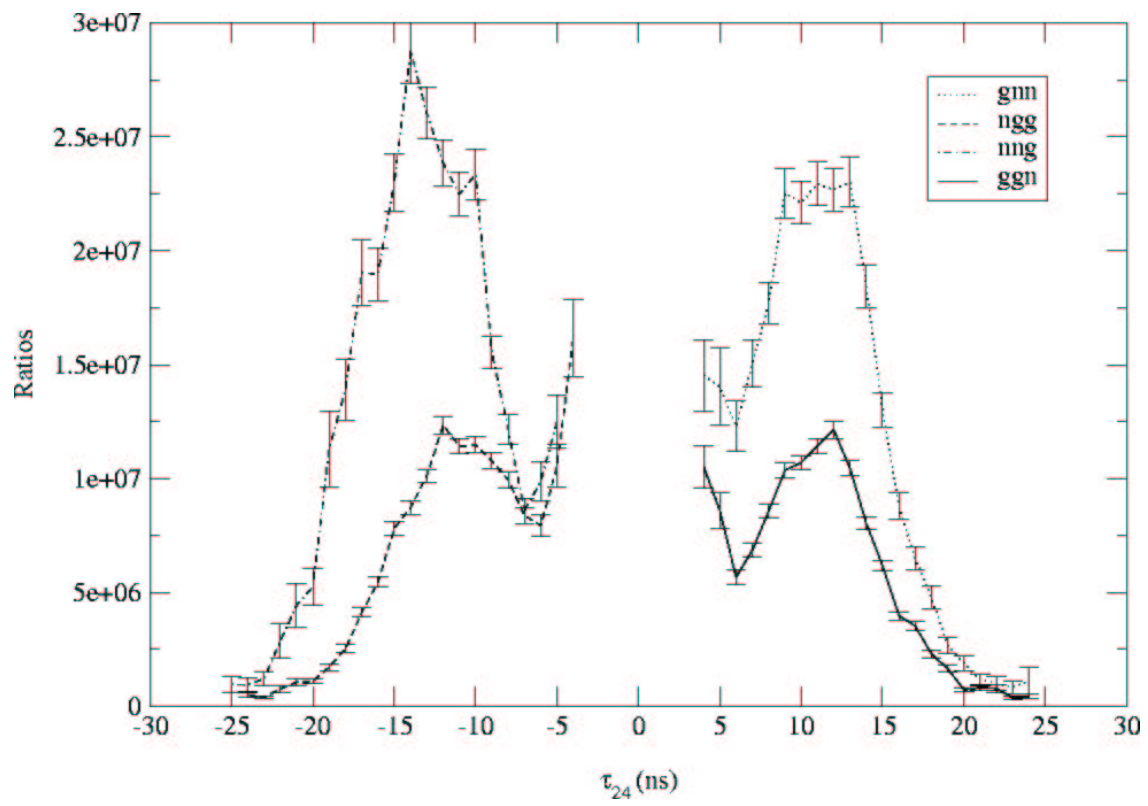

Figure 48: Ratio of $C_{n g}\left(\tau_{24}\right)^{2}$ with $C_{n g \mid n}\left(\tau_{24}\right)$ and $C_{n g \mid g}\left(\tau_{24}\right)$ on the left and ratio of $C_{g n}\left(\tau_{24}\right)^{2}$ with $C_{g n \mid n}\left(\tau_{24}\right)$ and $C_{g n \mid g}\left(\tau_{24}\right)$ on the right. Equations (135) and (136).

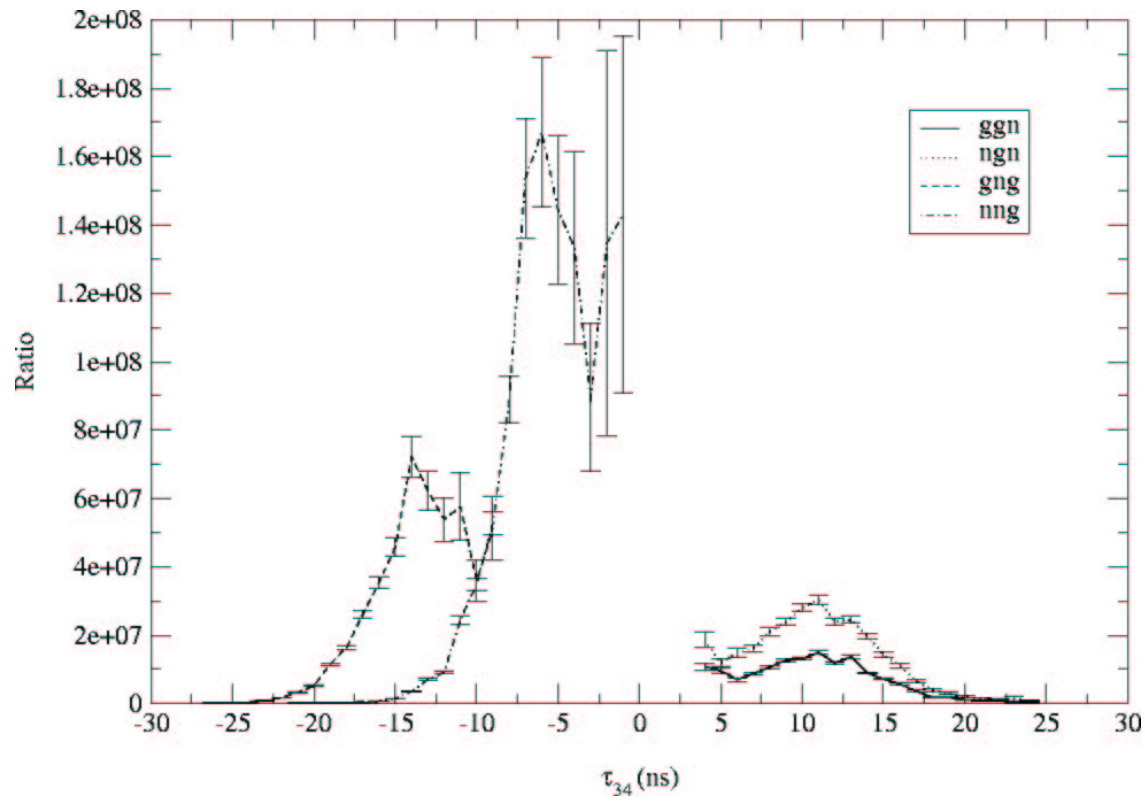

Figure 49: Ratio of $C_{n g}\left(\tau_{34}\right)^{2}$ with $C_{n g \mid n}\left(\tau_{34}\right)$ and $C_{n g \mid g}\left(\tau_{34}\right)$ on the left and ratio of $C_{g n}\left(\tau_{34}\right)^{2}$ with $C_{g n \mid n}\left(\tau_{34}\right)$ and $C_{g n \mid g}\left(\tau_{34}\right)$ on the right from Equations (135) and (136). 
effect, the 4 -inch detector should have about a $16 / 9=1.78$ advantage over the 3 -inch detectors. The detection efficiency for neutrons from Table 16 reflects this solid angle effect but the gamma detection efficiency does not.

Dead-time can be dismissed as an explanation for this anomaly. Assuming that the gamma-ray detection efficiency is $1.05 \times 10^{-3}$, the average number of detections per fission would be $\bar{\gamma} \epsilon_{g}=(7.98)\left(1.05 \times 10^{-3}\right)=0.008$. From Equation (113) in Chapter 6 we know that less than $\frac{\overline{\gamma(\gamma-1)}}{2} \epsilon_{g}^{2}=\frac{(68.15)}{2}\left(1.05 \times 10^{-3}\right)^{2}=3.8 \times 10^{-5}$ are lost to dead-time. This loss is less than $0.5 \%$.

A comparison between the time domain version of Equation 129 is shown in Figure 50. This comparison is consistent with equal detection efficiency between the two sizes of detectors. The ratio is nearly identical for both sizes of detectors. A similar comparison between the 3-inch and 4-inch detectors is shown in Figure 51 for the determination of the neutron detection efficiency from Equation 130. In this case the ratio reflects the expected effect of solid angle.

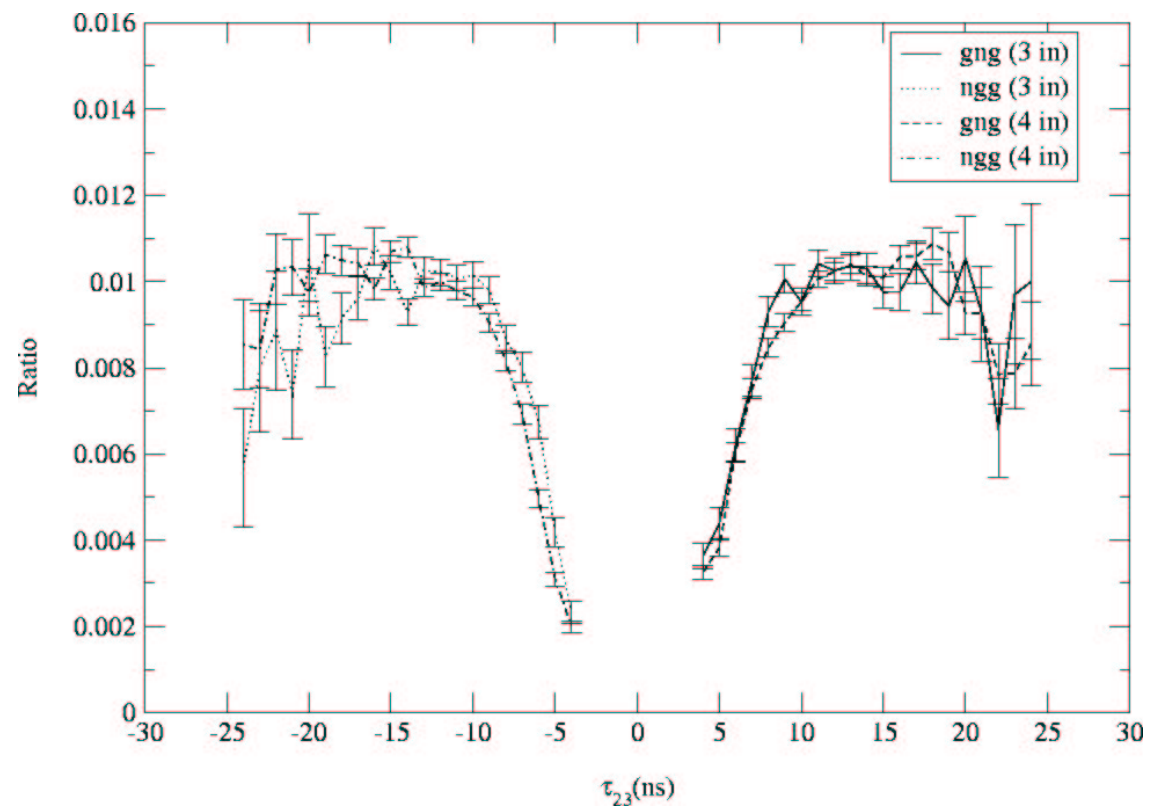

Figure 50: Ratio of $C_{n g \mid g}\left(\tau_{23}\right)$ with $C_{n g}\left(\tau_{23}\right)$ on the left and ratio of $C_{g n \mid g}\left(\tau_{23}\right)$ with $C_{g n}\left(\tau_{23}\right)$ on the right from Equation (133).

The determination of gamma-ray detection efficiency form Equation 129 depends also on 


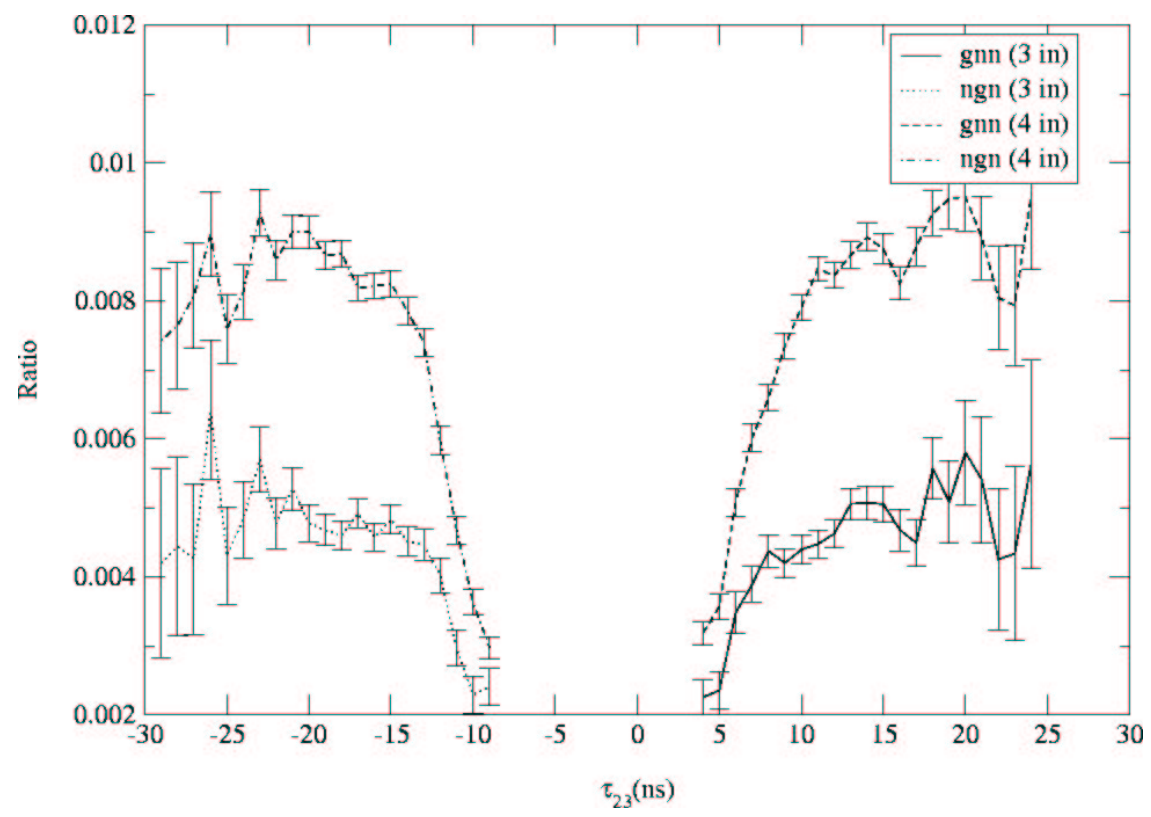

Figure 51: Ratio of $C_{n g \mid n}\left(\tau_{23}\right)$ with $C_{n g}\left(\tau_{23}\right)$ on the left and ratio of $C_{g n \mid n}\left(\tau_{23}\right)$ with $C_{g n}\left(\tau_{23}\right)$ on the right from Equations (134).

the first and second moments of $\gamma$. The source to detector correlation $C_{1 j}$ depends only on the first moment of $\gamma$ and detection efficiency. This correlation for all three of the 3 -inch and 4-inch detectors is shown in Figure 52. The neutron distributions are consistent with the difference in solid angle between the 3 -inch and 4-inch detectors. The gamma distributions at about $1 \mathrm{~ns}$ do not reflect the effect of solid angle. A closer view of the gamma distribution is shown in Figure 53. It is gratifying that the amplitude of the gamma distributions in Figure 53 is consistent with the gamma-ray detection efficiencies from Equation 129 in Table 15. The gamma-ray and neutron detection efficiencies computed from $C_{1 j}$ are shown in Table 18.

From a comparison at a delay beyond $20 \mathrm{~ns}$, it appears that the threshold energy for neutrons was slightly lower for the 4-inch detectors than for the 3 -inch detectors. For a given threshold on the CFD, the energy threshold for neutrons is higher than that for gamma rays.[10][12] This relation does not generally depend on detector size.

The 3-inch detectors detect about 1.6 gamma rays per neutron from fission while the 4-inch detectors detect about 0.94 gamma rays per neutron. Although the gamma-ray 
Table 18: Comparison of 3-inch and 4-inch detector efficiencies.

\begin{tabular}{ccccc}
\hline & \multicolumn{2}{c}{3 -inch } & \multicolumn{2}{c}{ 4-inch } \\
\cline { 2 - 5 }$k$ & $\epsilon_{n}$ & $\epsilon_{g}$ & $\epsilon_{n}$ & $\epsilon_{g}$ \\
\hline 2 & $1.45 \mathrm{E}-03$ & $1.05 \mathrm{E}-03$ & $2.90 \mathrm{E}-03$ & $1.31 \mathrm{E}-03$ \\
3 & $1.50 \mathrm{E}-03$ & $1.16 \mathrm{E}-03$ & $2.87 \mathrm{E}-03$ & $1.23 \mathrm{E}-03$ \\
4 & $1.47 \mathrm{E}-03$ & $1.15 \mathrm{E}-03$ & $2.71 \mathrm{E}-03$ & $1.21 \mathrm{E}-03$ \\
\hline
\end{tabular}

detections per fission between the 3-inch and 4-inch detectors are closer than expected, they are not as close as indicated by the passive estimation of gamma ray detection efficiency from Table 15. The ratio of the two is $\frac{C_{1 j g 4-i n}}{C_{1 j g 3-i n}}=1.11$.

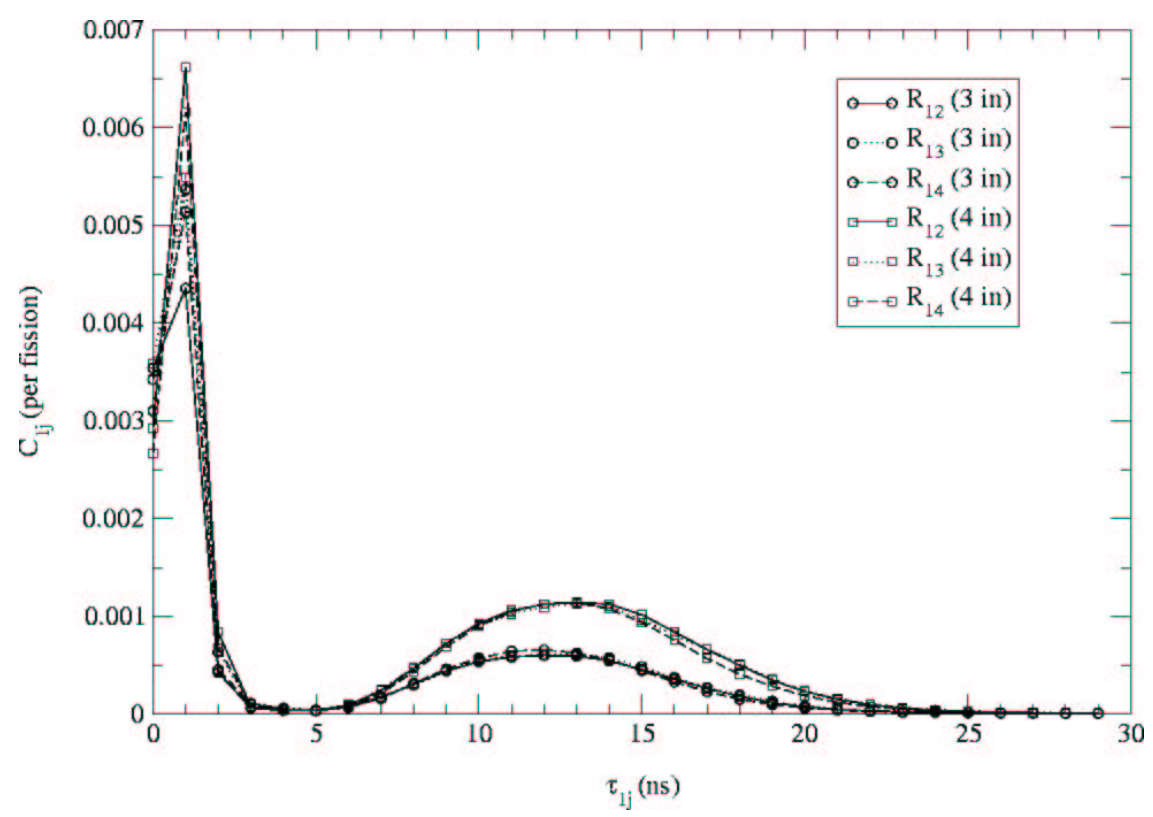

Figure 52: Comparison of the source-detector correlations $C_{1 j}\left(\tau_{1 j}\right)$ in correlated counts per fission between the 3 -inch and 4 -inch detectors.

A comparison between the 3-inch and 4-inch detectors of the detector-detector correlation $C_{i j}$ is shown in Figure 54 with a closeup of the $g g$ peak in in Figure 55. This correlation is proportional to the square of the detection efficiency and the second multiplicity moment. In general it is consistent with the previous discussion. The ratio of the 


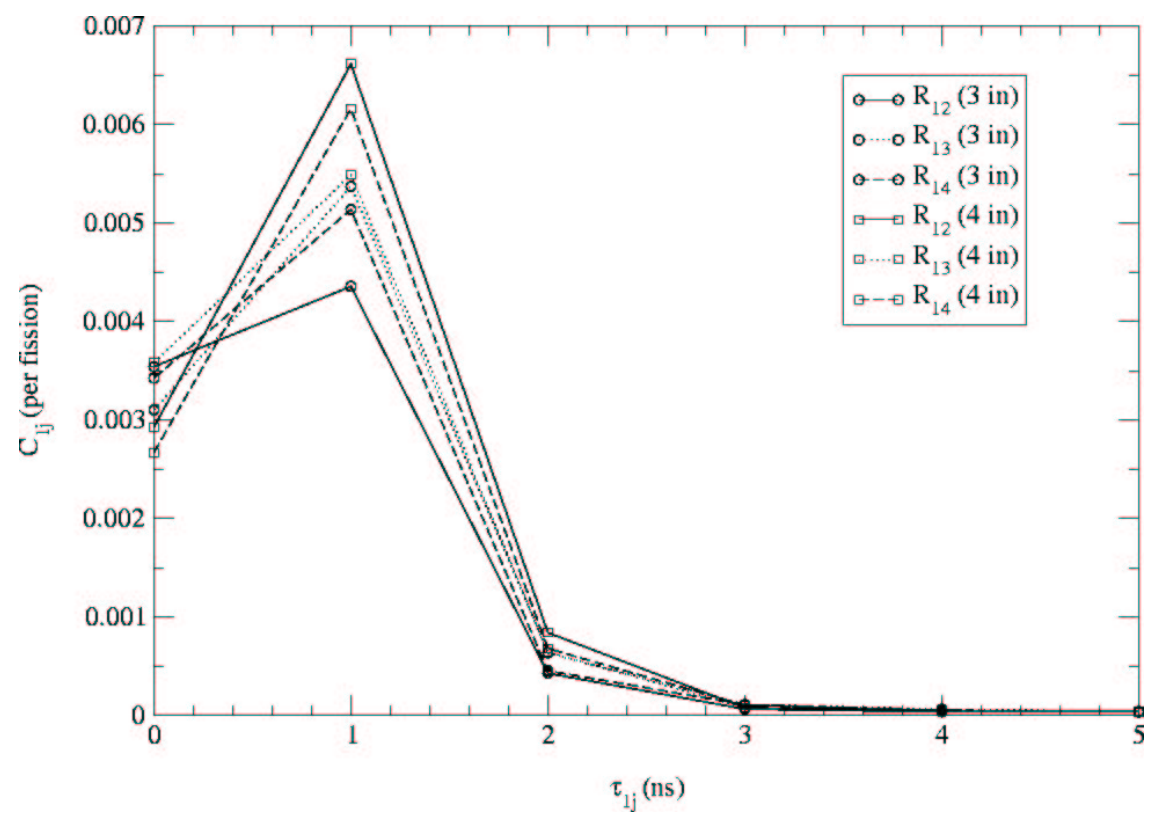

Figure 53: Comparison of the gamma peak of the sourcedetector correlations $C_{1 j}\left(\tau_{1 j}\right)$ in correlated counts per fission between the 3 -inch and 4 -inch detectors.

two is $\frac{C_{i j g 4-i n}}{C_{i j g 3-i n}}=1.54$. This ratio indicates that the detection efficiency for the 4-inch detectors for gamma rays from all sources is 1.24 times that of the 3-inch detectors. Recall however that for gamma rays, the detector-detector correlation may contain gamma pairs from sources other than fission.

The detection efficiency for gamma rays was lower for the 4-inch detectors than would be expected from both solid angle effects and neutron detection efficiency. In addition, the passive method from Equation 129 to determine gamma ray detection efficiency was lower than indicated from the source-to-detector correlation $C_{1 j}$. Underestimating gamma-ray detection efficiency will result in an overestimate in the ${ }^{252} \mathrm{Cf}$ mass from Equation 132.

\subsection{Determination of mass from gamma-neutron-neutron triplets}

An alternative to Equations (131) and (132) may be better to determine the number of spontaneous fissions. With $\epsilon_{n}$ and $\epsilon_{g}$ determined from $C_{1 j}$ in Section 8.3, the number of fissions are proportional to $C_{g n n}$. The neutron pairs assures that the neutron source is not from $(\alpha, n)$ reactions.[14] Furthermore, the gnn regions are more temporally distinct than 


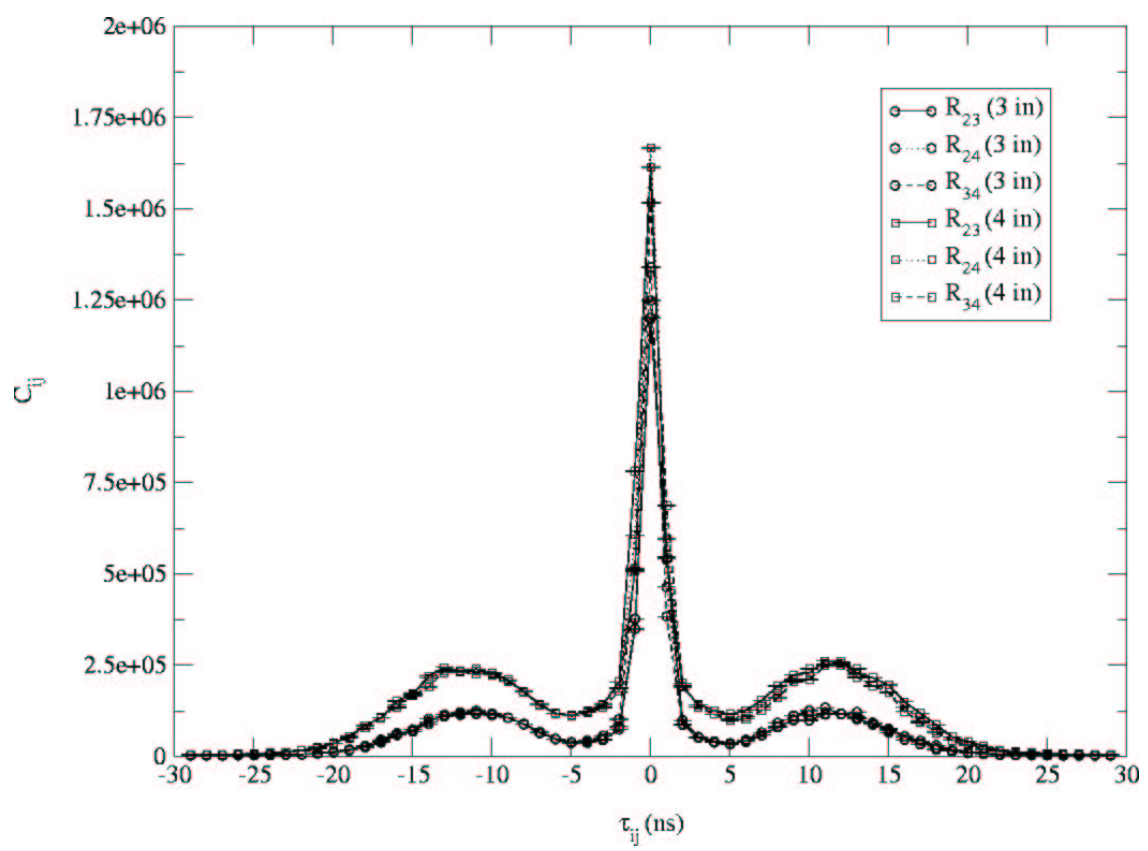

Figure 54: Comparison of the detector-detector correlations $C_{i j}\left(\tau_{i j}\right)$ in total correlated counts between the 3 -inch and 4inch detectors.

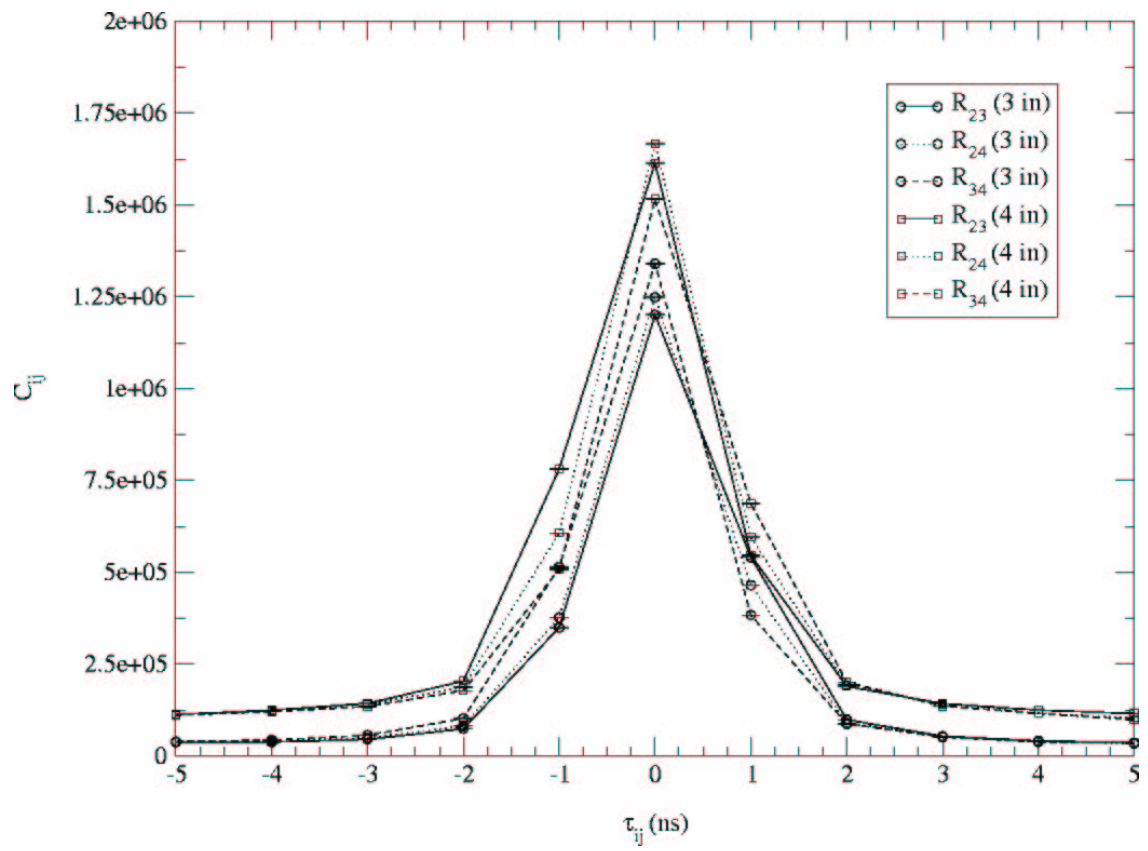

Figure 55: Comparison of the gamma peak of the detectordetector correlations $C_{i j}\left(\tau_{i j}\right)$ in total correlated counts between the 3 -inch and 4 -inch detectors. 
the $n n$ pairs in $C_{n n}$. The equation for this correlation is

$$
C_{g n \mid n}=N \bar{\gamma} \overline{\nu(\nu-1)} \epsilon_{n}^{2} \epsilon_{g}
$$

As before, each of the three regions $g n n, n g n$ and $n n g$ can be integrated with respect to one time variable leaving the correlation a function of the other time variable. For example the region gnn can be integrated on $\tau_{23}$ leaving it as a function of $\tau_{24}$ and vice versa. The six combinations are shown in Figure 56. Better results are obtained if the nnn triplets are subtracted from the data. From Figure 56 one can see the the neutron triplet rate at $\tau=5 \mathrm{~ns}$ is 89 counts per 1 ns time bin. The total number of neutron triplets in the gnn region is shown in Table 19 along with the results for each of the three regions gnn, ngn and nng. The equations for each of these three regions are shown below with the appropriate detector efficiencies used.

$$
\begin{aligned}
& C_{g n n}=N \overline{\nu(\nu-1)} \bar{\gamma} \epsilon_{g 2} \epsilon_{n 3} \epsilon_{n 4} \\
& C_{n g n}=N \overline{\nu(\nu-1)} \bar{\gamma} \epsilon_{g 3} \epsilon_{n 2} \epsilon_{n 4} \\
& C_{n n g}=N \overline{\nu(\nu-1)} \bar{\gamma} \epsilon_{g 4} \epsilon_{n 2} \epsilon_{n 3}
\end{aligned}
$$

The results from these equations are presented in Table 19. The value of $C_{i j k}$ is shown with the $n n n$ contribution subtracted out. The detection efficiencies are from Table 18. The counts in $C_{i j k}$, nnn and accidentals contributed to the estimate of the uncertainty. The relative uncertainty for the 4-inch detector is less because of the greater count rate. Both the $g n n$ and $n g n$ regions produced very consistent results. The $n n g$ region produced slightly lower estimates for the number of fissions for both the 3-inch and 4-inch detectors.

The ${ }^{252} C f$ source was independently measured at ORNL on October $18,2002^{1}$ by comparing total neutron count from ${ }^{3} \mathrm{He}$ detectors to a NIST traceable ${ }^{252} \mathrm{Cf}$ source. This measurement resulted in $4.6 \times 10^{4} \pm 3 \%$ fissions per second for a ${ }^{252} C f$ mass of $0.0749 \mu \mathrm{g}$. This measurement implies a ${ }^{252} C f$ mass of $0.115 \mu \mathrm{g}$ and $0.114 \mu \mathrm{g}$ on the dates of the 3 -inch

\footnotetext{
${ }^{1}$ Personal communication with John Neal, John Mihalczo and Sara Pozzi.
} 


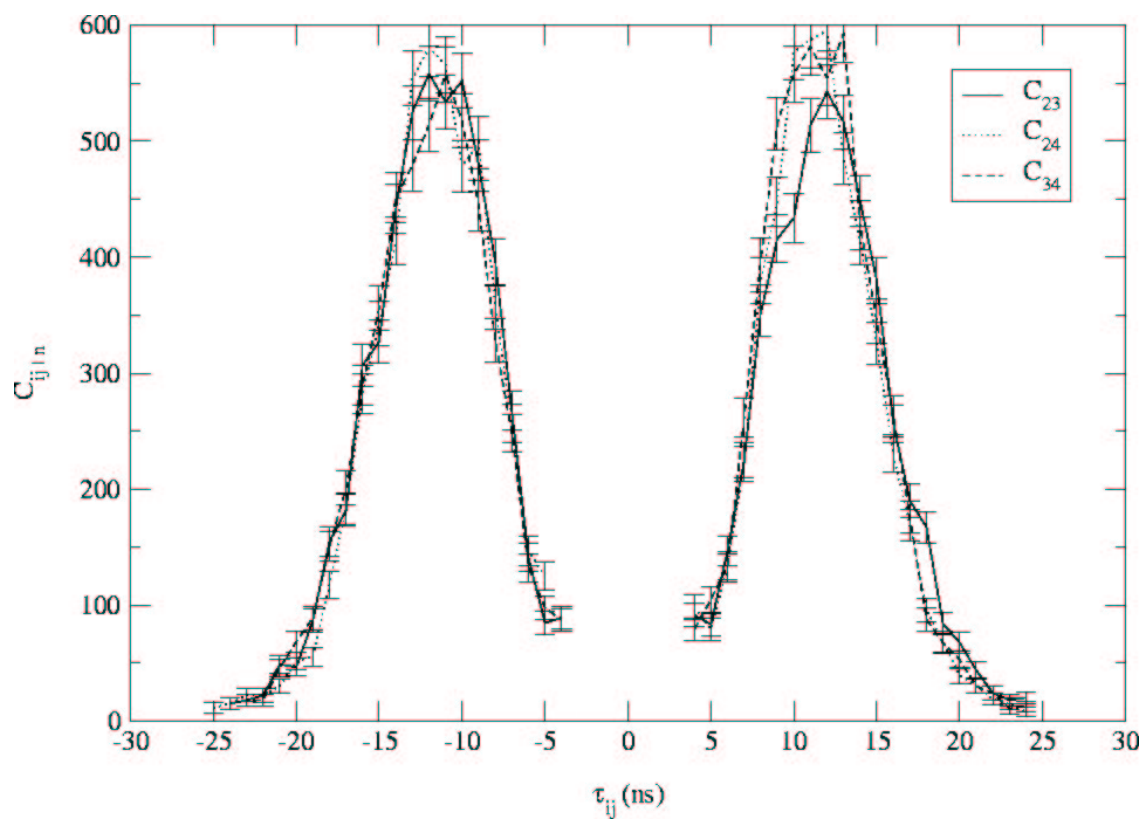

Figure 56: Gamma-neutron-neutron triplets as a function of delay time $\tau_{i j}$ given that a neutron was detected in the third detector $k$.

Table 19: Determination of the number of spontaneous fissions $N$ from Equation (132) from the 3-inch and 4-inch detectors.

\begin{tabular}{|c|c|c|c|c|c|c|c|c|}
\hline \multirow[b]{2}{*}{ triplet } & \multicolumn{4}{|c|}{3 -in } & \multicolumn{4}{|c|}{ 4-in } \\
\hline & $C_{i j k}$ & $N$ & $\begin{array}{l}\frac{\sigma_{N}}{N} \\
(\%)\end{array}$ & $\begin{array}{l}m_{c f} \\
(\mu g)\end{array}$ & $C_{i j k}$ & $N$ & $\begin{array}{l}\frac{\sigma_{N}}{N} \\
(\%)\end{array}$ & $\begin{array}{l}m_{c f} \\
(\mu g)\end{array}$ \\
\hline$n n n$ & 895 & - & - & - & 3940 & - & - & - \\
\hline$g n n$ & $4.12 \mathrm{E}+03$ & $1.85 \mathrm{E}+10$ & $1.7 \%$ & 0.118 & $1.77 \mathrm{E}+04$ & $1.80 \mathrm{E}+10$ & $0.8 \%$ & 0.115 \\
\hline$n g n$ & $4.38 \mathrm{E}+03$ & $1.84 \mathrm{E}+10$ & $1.7 \%$ & 0.117 & $1.68 \mathrm{E}+04$ & $1.81 \mathrm{E}+10$ & $0.9 \%$ & 0.115 \\
\hline$n n g$ & $4.22 \mathrm{E}+03$ & $1.75 \mathrm{E}+10$ & $1.7 \%$ & 0.112 & $1.72 \mathrm{E}+04$ & $1.78 \mathrm{E}+10$ & $0.8 \%$ & 0.113 \\
\hline
\end{tabular}


and 4-inch experiments respectively. The results from the $C_{g n \mid n}$ method in Table 19 are consistent with this independent measurement. 


\section{CHAPTER IX}

\section{CONCLUSIONS}

A new, passive method to measure the mass of a spontaneous fission source with fast-plastic scintillating detectors was developed. It was demonstrated for the simple case of a nonmultiplying point source provided by a ${ }^{252} C f$ source. The method is based on physical principles rather than a calibration. Furthermore, it is possible to determine detection efficiency from the measurement of the unknown. Therefore calibration and independent determination of detector efficiency are not necessary.

The analysis is aided by the temporal separation of gamma-neutron combinations. However, even for a point source, the gamma-neutron temporal separation was not adequate to provide perfect separation. However, removing $n n n$ triplets from the $g n n$ combinations provided an accurate measure of fissile mass.

The mass of the ${ }^{252} \mathrm{Cf}$ source was estimated from three methods. The results of these methods are compared to an independent measurement of the source at ORNL in Table 20. The three methods include 1) the count rate of the ion-chamber containing the source; 2) ratios of $\frac{C_{g n}^{2}}{C_{g n \mid g}}$ and $\frac{C_{g n}^{2}}{C_{g n \mid n}}$; and 3) the $C_{g n \mid n}$ correlations.

The ${ }^{252} C f$ source was independently measured at ORNL on October $18,2002^{1}$ by comparing total neutron count from ${ }^{3} \mathrm{He}$ detectors to a NIST traceable ${ }^{252} \mathrm{Cf}$ source. This measurement resulted in $4.6 \times 10^{4} \pm 3 \%$ fissions per second for a ${ }^{252} C f$ mass of $0.0749 \mu \mathrm{g}$. This measurement implies a ${ }^{252} C f$ mass of $0.115 \mu \mathrm{g}$ and $0.114 \mu \mathrm{g}$ on the dates of the 3 -inch and 4-inch experiments respectively.

The number of fissions detected in the ion-chamber containing the ${ }^{252} \mathrm{Cf}$, adjusted for the fission detection-efficiency $\epsilon_{f}$ is the most reliable of these. Of the two passive methods, the $C_{g n \mid n}$ correlation proved to be more accurate with an adjustment for neutron triplets.

\footnotetext{
${ }^{1}$ Personal communication with John Neal, John Mihalczo and Sara Pozzi.
} 
The ratio of the $g n$ correlation squared suffered from both $n n$ contamination in $C_{g n}$ as well as $n n n$ contamination in $C_{g n \mid g}$ and $C_{g n \mid n}$. The results for each of these methods to determine the ${ }^{252} \mathrm{Cf}$ mass are shown below in Table 20 for both the 3 -inch and 4 -inch detector experiments.

Table 20: Summary of the ${ }^{252} C f$ mass determination from various methods

\begin{tabular}{llll}
\hline & & 3-inch detectors & 4-inch detectors \\
\cline { 3 - 4 } Method & Equation & $\begin{array}{c}{ }^{252} C f \text { mass } \\
(\mu \mathrm{g})\end{array}$ & $\begin{array}{c}{ }^{252} C f \text { mass } \\
(\mu \mathrm{g})\end{array}$ \\
\hline Independent & - & $0.115 \pm 3 \%$ & $0.114 \pm 3 \%$ \\
Ion-Chamber & Eq. $(55)$ & $0.128 \pm 10 \%$ & $0.127 \pm 10 \%$ \\
$C_{g n}^{2}$ ratio & Eq. $(131),(132)$ & $0.18 \pm 7 \%$ & $0.22 \pm 7 \%$ \\
$C_{g n \mid n}$ & Eq. $(137)$ & $0.117 \pm 1.7 \%$ & $0.115 \pm 0.9 \%$ \\
\hline
\end{tabular}

The analysis developed in Chapter 4 provides the tools to determine both the quantity of a fission source as well as detector efficiency in a single measurement without the necessity of a calibration standard. The temporal separation between gamma-neutron combinations will be degraded in more complex measurement scenarios by scattering, spatial distribution of the fissile material and multiplication. In these cases greater care will be required to separate overlapping regions. The analytical tools necessary to do this were developed in Sections 4.5 and 5.1. In any event, the analysis developed in Chapter 4 with the extension to multiplying material in Chapter 5 are perfectly valid without temporal separation.

In addition to the temporal effects, correlated detections can result from both elastic and inelastic scattering, radioactive decay and alpha-n reactions. The analysis of the time correlations was developed to include these interactions in Section 4.6. The moments for each of these are summarized in Table 7. In more complex measurement scenarios, greater care in the analysis may be required to separate these correlations. The gnn combinations as used in this experiment to determine the ${ }^{252} \mathrm{Cf}$ mass appears to be the most immune from these interfering interactions.

Larger detectors are of course desirable to make this method more practical. Larger 
detectors will reduce measurement time. On the other hand, larger detectors will be subject to greater cross-talk and more dead-time as count rates increase. This advantage must also be weighed against practical considerations.

Because of the extremely short correlation time allowed by this method, (one thousandth that of a well counter) this technique should prove superior to well counters in the measurement of oxides and fluorides where the background from $(\alpha, \mathrm{n})$ reactions is troublesome.

The next experiment using this method might be the measurement of a thin spherical shell of $\mathrm{Pu}$. This experiment will provide the extension of a spatial distribution of a different fissile material while minimizing the effects of attenuation and multiplication. 


\section{APPENDIX A}

\section{FACTORIAL MOMENTS FROM PROBABILITY GENERATING FUNCTIONS}

\section{$\underline{\text { A.1 Definitions }}$}

In this appendix, probability generating functions will be used to generate the factorial moments for gamma and neutron counting from fission for multiplying media. First the moments used in Chapter 4 will be derived using probability generating functions. The effect of multiplication will then be included in the equation and the moments re-derived to include the effect of self multiplication.

The number of neutrons produced in a fission reaction is a random variable $\nu$ with a probability density $p_{\nu}(\nu)$. A similar statement can be made about the number of gamma rays $\gamma$ and $p_{\gamma}(\gamma)$. The probability generating function for these discrete random variables is

$$
p_{\nu}(z)=\sum_{\nu=0}^{\infty} p_{\nu}(\nu) z^{\nu}
$$

and

$$
p_{\gamma}(z)=\sum_{\gamma=0}^{\infty} p_{\gamma}(\gamma) z^{\gamma}
$$

respectively.

The $n$th moment $\nu_{n}$ of the random variable is

$$
\nu_{n}=\left.\frac{d^{n} p_{\nu}(z)}{d z^{n}}\right|_{z=1}
$$


The zeroth and first three moments of $\nu$ are then

$$
\begin{aligned}
& \nu_{0}=\left.\sum_{\nu=0}^{\infty} p_{\nu}(\nu) z^{\nu}\right|_{z=1}=1 \\
& \nu_{1}=\left.\frac{d p_{\nu}(z)}{d z}\right|_{z=1}=\left.\sum_{\nu=0}^{\infty} \nu p_{\nu}(\nu) z^{\nu-1}\right|_{z=1}=\bar{\nu} \\
& \nu_{2}=\left.\frac{d^{2} p_{\nu}(z)}{d z^{2}}\right|_{z=1}=\left.\sum_{\nu=0}^{\infty} \nu(\nu-1) p_{\nu}(\nu) z^{\nu-2}\right|_{z=1}=\overline{\nu(\nu-1)} \\
& \nu_{3}=\left.\frac{d^{3} p_{\nu}(z)}{d z^{3}}\right|_{z=1}=\left.\sum_{\nu=0}^{\infty} \nu(\nu-1)(\nu-2) p_{\nu}(\nu) z^{\nu-3}\right|_{z=1}=\overline{\nu(\nu-1)(\nu-2)}
\end{aligned}
$$

Of course the same goes for $\gamma$.

\section{A.2 Combined $\gamma, \nu$ moments}

The combined $\gamma, \nu$ moments can also be derived by forming a new random variable $n=\nu+\gamma$. The probability generating function for $n$ is

$$
p_{n}(z)=p_{\nu}(z) p_{\gamma}(z) .
$$

One can again write the factorial moments for the combined gamma-neutron detection. Beginning with the 0th moment

$$
\begin{aligned}
n_{0} & =\left.p_{\nu}(z) p_{\gamma}(z)\right|_{z=1} \\
& =\left.\sum_{\nu=0}^{\infty} p_{\nu}(\nu) z^{\nu} \sum_{\gamma=0}^{\infty} p_{\gamma}(\gamma) z^{\gamma}\right|_{z=1}=1
\end{aligned}
$$

The first moment of the combined gamma-neutron detection is

$$
\begin{aligned}
n_{1} & =\left.\frac{d p_{n}}{d z}\right|_{z=1} \\
& =\frac{d p_{\nu}}{d z} p_{\gamma}+\left.\frac{d p_{\gamma}}{d z} p_{\nu}\right|_{z=1}
\end{aligned}
$$

where the functional $(z)$ was dropped to save on clutter. 
The second moment is then found by taking the derivative again

$$
\begin{aligned}
n_{2}= & \left.\frac{d^{2} p_{n}}{d z^{2}}\right|_{z=1} \\
& =\frac{d^{2} p_{\nu}}{d z^{2}} p_{\gamma}+\frac{d p_{\nu}}{d z} \frac{d p_{\gamma}}{d z}+\frac{d^{2} p_{\gamma}}{d z^{2}} p_{\nu}+\left.\frac{d p_{\gamma}}{d z} \frac{d p_{\nu}}{d z}\right|_{z=1} \\
& =\frac{d^{2} p_{\nu}}{d z^{2}} p_{\gamma}+2 \frac{d p_{\nu}}{d z} \frac{d p_{\gamma}}{d z}+\left.\frac{d^{2} p_{\gamma}}{d z^{2}} p_{\nu}\right|_{z=1} \\
n_{3}= & \left.\frac{d^{3} p_{n}}{d z^{3}}\right|_{z=1} \\
= & \frac{d^{3} p_{\nu}}{d z^{3}} p_{\gamma}+\frac{d^{2} p_{\nu}}{d z^{2}} \frac{d p_{\gamma}}{d z} \\
+ & \frac{d^{2} p_{\nu}}{d z^{2}} \frac{d p_{\gamma}}{d z}+\frac{d p_{\nu}}{d z} \frac{d^{2} p_{\gamma}}{d z^{2}} \\
+ & \frac{d^{3} p_{\gamma}}{d z^{3}} p_{\nu}+\frac{d^{2} p_{\gamma}}{d z^{2}} \frac{d p_{\nu}}{d z} \\
+ & \frac{d^{2} p_{\gamma}}{d z^{2}} \frac{d p_{\nu}}{d z}+\left.\frac{d p_{\gamma}}{d z} \frac{d^{2} p_{\nu}}{d z^{2}}\right|_{z=1} p_{\gamma}+3 \frac{d^{2} p_{\nu}}{d z^{2}} \frac{d p_{\gamma}}{d z}+3 \frac{d p_{\nu}}{d z} \frac{d^{2} p_{\gamma}}{d z^{2}}+\left.\frac{d^{3} p_{\gamma}}{d z^{3}} p_{\nu}\right|_{z=1}
\end{aligned}
$$

Now substituting the non-multiplying moments from Equation (144) for the values of the various derivatives yields

$$
\begin{aligned}
n_{1} & =\bar{\nu}+\bar{\gamma} \\
n_{2} & =\overline{\nu(\nu-1)}+\overline{\nu \gamma}+\overline{\gamma(\gamma-1)}+\overline{\gamma \nu} \\
n_{3} & =\overline{\nu(\nu-1)(\nu-2)}+\overline{\nu(\nu-1)} \bar{\gamma} \\
& +\overline{\nu(\nu-1)} \bar{\gamma}+\overline{\nu \gamma(\gamma-1)} \\
& +\overline{\gamma(\gamma-1)(\gamma-2)}+\overline{\gamma(\gamma-1)} \bar{\nu} \\
& +\overline{\gamma(\gamma-1)} \bar{\nu}+\overline{\gamma(\nu-1)} .
\end{aligned}
$$


The moments for the four regions in the detector-detector correlation and the eight regions in the bicorrelation are produced in the resulting Equation (150).

\section{A.3 Multiplication and neutron moments}

Böhnel introduces the probability generating function $h(z)$ to account for multiplication. It represents the number of neutrons leaving the system after the introduction of a single neutron

$$
h(z)=(1-p) z+p p_{\nu_{i}}[h(z)]
$$

where $p$ is the probability that a neutron induces a fission. The subscript $i$ on $\nu_{i}$ indicates the multiplicity of an induced fission.

The first three derivatives of $h(z)$ are

$$
\begin{aligned}
\frac{d h(z)}{d z} & =(1-p)+p \frac{d p_{\nu_{i}}[h]}{d h} \frac{d h(z)}{d z} \\
\frac{d^{2} h(z)}{d z^{2}} & =p\left[\frac{d^{2} p_{\nu_{i}}[h]}{d h^{2}}\left(\frac{d h(z)}{d z}\right)^{2}+\frac{d p_{\nu_{i}}[h]}{d h} \frac{d^{2} h(z)}{\left.d z^{2}\right]}\right. \\
\frac{d^{3} h(z)}{d z^{3}} & =p\left[\frac{d^{3} p_{\nu_{i}}[h]}{d h^{3}}\left(\frac{d h(z)}{d z}\right)^{3}\right. \\
& \left.+3 \frac{d^{2} p_{\nu_{i}}[h]}{d h^{2}} \frac{d h(z)}{d z} \frac{d^{2} h(z)}{d z^{2}}+\frac{d p_{\nu_{i}}[h]}{d h} \frac{d^{3} h(z)}{d z^{3}}\right] .
\end{aligned}
$$

Notice that the derivatives of $h(z)$ with respect to $z$ appear on both sides of the equation. The derivatives can now be solved and evaluated at $z=1$. It should be considered that $\left.h(z)\right|_{z=1}=1$. Therefore

$$
\left.\frac{d p_{\nu_{i}}[h(z)]}{d z}\right|_{z=1}=\left.\frac{d p_{\nu_{i}}[h]}{d h}\right|_{h=1}=\overline{\nu_{i}}
$$

etc.

The derivatives of $h(z)$ can now be explicitly solved for later use.

$$
\begin{aligned}
&\left.\frac{d h(z)}{d z}\right|_{z=1}=\frac{(1-p)}{1-p \overline{\nu_{i}}} \\
&\left.\frac{d^{2} h(z)}{d z^{2}}\right|_{z=1}=\frac{p(1-p)^{2} \overline{\nu_{i}\left(\nu_{i}-1\right)}}{\left(1-p \overline{\nu_{i}}\right)^{3}} \\
&\left.\frac{d^{3} h(z)}{d z^{3}}\right|_{z=1}=\frac{p(1-p)^{3}}{\left(1-p \overline{\nu_{i}}\right)^{4}}\left[\overline{\nu_{i}\left(\nu_{i}-1\right)\left(\nu_{i}-2\right)}+3{\overline{\nu_{i}\left(\nu_{i}-1\right)}}^{2} \frac{p}{1-p \overline{\nu_{i}}}\right]
\end{aligned}
$$


The function $h(z)$ represents the response of the system to the introduction of a single neutron. The function $\nu(z)=f_{s}[h(z)]$ represents the response of the system to a spontaneous fission source. The process of taking derivatives is now repeated with $\nu(z)$.

$$
\begin{aligned}
\frac{d \nu(z)}{d z} & =\frac{d f_{s}}{d h} \frac{d h}{d z} \\
\frac{d^{2} \nu(z)}{d z^{2}} & =\frac{d^{2} f_{s}}{d h^{2}}\left(\frac{d h}{d z}\right)^{2}+\frac{d f_{s}}{d h} \frac{d^{2} h}{d z^{2}} \\
\frac{d^{3} \nu(z)}{d z^{3}} & =\frac{d^{3} f_{s}}{d h^{3}}\left(\frac{d h}{d z}\right)^{3}+3 \frac{d^{2} f_{s}}{d h^{2}} \frac{d h}{d z} \frac{d^{2} h}{d z^{2}}+\frac{d f_{s}}{d h} \frac{d^{3} h}{d z^{3}}
\end{aligned}
$$

The equations are again solved for $z=1$.

$$
\begin{aligned}
\left.\frac{d \nu(z)}{d z}\right|_{z=1} & =\left(\frac{1-p}{1-p \overline{\nu_{i}}}\right) \overline{\nu_{s}} \\
\left.\frac{d^{2} \nu(z)}{d z^{2}}\right|_{z=1} & =\left(\frac{1-p}{1-\nu_{i} p}\right)^{2}\left[\overline{\nu_{s}\left(\nu_{s}-1\right)}+\frac{p \overline{\nu_{i}\left(\nu_{i}-1\right)}}{1-\nu_{i} p} \overline{\nu_{s}}\right] \\
\left.\frac{d^{3} \nu(z)}{d z^{3}}\right|_{z=1} & =\left(\frac{1-p}{1-\nu_{i} p}\right)^{3}\left\{\overline{\nu_{s}\left(\nu_{s}-1\right)\left(\nu_{s}-2\right)}\right. \\
& +\frac{p}{1-\nu_{i} p}\left[3 \overline{\nu_{i}\left(\nu_{i}-1\right) \nu_{s}\left(\nu_{s}-1\right)}+\overline{\nu_{i}\left(\nu_{i}-1\right)\left(\nu_{i}-2\right)} \overline{\nu_{s}}\right] \\
& \left.+3\left(\frac{p}{1-\nu_{i} p}\right)^{2} \overline{\nu_{i}\left(\nu_{i}-1\right)} \bar{\nu}^{2}\right\}
\end{aligned}
$$

Usually Equation (156) is written in terms of the leakage multiplication $M$ rather than the variable $p$. Where the leakage multiplication is defined as

$$
M=\frac{1-p}{1-p \overline{\nu_{i}}}
$$

The other factor involving $p$ then becomes

$$
\frac{p}{1-p \overline{\nu_{i}}}=\frac{M-1}{\overline{\nu_{i}}-1}
$$

With these two substitutions and the substitution $\nu_{n}$ for the $n$th factorial moment 
Equation (156) becomes

$$
\begin{aligned}
\left.\frac{d \nu(z)}{d z}\right|_{z=1} & =M \nu_{s 1} \\
\left.\frac{d^{2} \nu(z)}{d z^{2}}\right|_{z=1} & =M^{2}\left[\nu_{s 2}+\left(\frac{M-1}{\nu_{i 1}-1}\right) \nu_{s 1} \nu_{i 2}\right] \\
\left.\frac{d^{3} \nu(z)}{d z^{3}}\right|_{z=1} & =M^{3}\left\{\nu_{s 3}+\left(\frac{M-1}{\overline{\nu_{i}}-1}\right)\left[3 \nu_{s 2} \nu_{i 2}+\nu_{s 1} \nu_{i 3}\right]\right. \\
& \left.+3\left(\frac{M-1}{\nu_{i 1}-1}\right)^{2} \nu_{s 1} \nu_{i 2}^{2}\right\}
\end{aligned}
$$

\section{A.4 Multiplication and gamma moments}

At this point the gamma rays must be considered. The average number of neutrons escaping from the sample from a single spontaneous fission and fissions induced thereby is $M \overline{\nu_{s}}$. The number of induced fissions can therfore be inferred.

$$
M \overline{\nu_{s}}=(1-p)\left[\overline{\nu_{s}}+f_{i} \overline{\nu_{i}}\right]
$$

Solving for $f_{i}$ in terms of $M$ and $\nu$ gives

$$
f_{i}=\frac{M-1}{\overline{\nu_{i}}-1} \overline{\nu_{s}}
$$

Now for a spontaneous fission, the total number of gamma rays generated will be

$$
\gamma=\gamma_{s}+f_{i} \gamma_{i}
$$

The probability generating function will be

$$
p_{\gamma}(z)=p_{\gamma_{s}}(z) p_{\gamma_{i}}\left(f_{i} z\right)
$$


Again the first three derivatives of $p_{\gamma}(z)$ are taken,

$$
\begin{aligned}
\frac{d p_{\gamma}}{d z} & =\frac{d p_{\gamma_{s}}}{d z} p_{\gamma_{i}}\left(f_{i} z\right)+p_{\gamma_{s}}(z) \frac{d p_{\gamma_{i}}}{d z} f_{i} \\
\frac{d^{2} p_{\gamma}}{d z^{2}} & =\frac{d^{2} p_{\gamma_{s}}}{d z^{2}} p_{\gamma_{i}}\left(f_{i} z\right)+\frac{d p_{\gamma_{s}}}{d z} \frac{d p_{\gamma_{i}}}{d z} f_{i} \\
& +\frac{d p_{\gamma_{s}}}{d z} \frac{d p_{\gamma_{i}}}{d z} f_{i}+p_{\gamma_{s}}(z) \frac{d^{2} p_{\gamma_{i}}}{d z^{2}} f_{i}^{2} \\
& =\frac{d^{2} p_{\gamma_{s}}}{d z^{2}} p_{\gamma_{i}}\left(f_{i} z\right)+2 \frac{d p_{\gamma_{s}}}{d z} \frac{d p_{\gamma_{i}}}{d z} f_{i}+p_{\gamma_{s}}(z) \frac{d^{2} p_{\gamma_{i}}}{d z^{2}} f_{i}^{2} \\
\frac{d^{3} p_{\gamma}}{d z^{3}} & =\frac{d^{3} p_{\gamma_{s}}}{d z^{3}} p_{\gamma_{i}}\left(f_{i} z\right)+\frac{d^{2} p_{\gamma_{s}}}{d z^{2}} \frac{d p_{\gamma_{i}}}{d z} f_{i} \\
& +\frac{d^{2} p_{\gamma_{s}}}{d z^{2}} \frac{d p_{\gamma_{i}}}{d z} f_{i}+\frac{d p_{\gamma_{s}}}{d z} \frac{d^{2} p_{\gamma_{i}}}{d z^{2}} f_{i}^{2} \\
& +\frac{d^{2} p_{\gamma_{s}}}{d z^{2}} \frac{d p_{\gamma_{i}}}{d z} f_{i}+\frac{d p_{\gamma_{s}}}{d z} \frac{d^{2} p_{\gamma_{i}}}{d z^{2}} f_{i}^{2} \\
& +\frac{d p_{\gamma_{s}}}{d z} \frac{d^{2} p_{\gamma_{i}}}{d z^{2}} f_{i}^{2}+p_{\gamma_{s}}(z) \frac{d^{3} p_{\gamma_{i}}}{d z^{3}} f_{i}^{3} \\
& =\frac{d^{3} p_{\gamma_{s}}}{d z^{3}} p_{\gamma_{i}}\left(f_{i} z\right)+3 \frac{d^{2} p_{\gamma_{s}}}{d z^{2}} \frac{d p_{\gamma_{i}}}{d z} f_{i}+3 \frac{d p_{\gamma_{s}}}{d z} \frac{d^{2} p_{\gamma_{i}}}{d z^{2}} f_{i}^{2}+p_{\gamma_{s}}(z) \frac{d^{3} p_{\gamma_{i}} f_{i}^{3}}{d z^{3}}
\end{aligned}
$$

and again solving at $z=1$ produces

$$
\begin{aligned}
\left.\frac{d p_{\gamma}}{d z}\right|_{z=1} & =\gamma_{s 1}+\left(\frac{M-1}{\nu_{i 1}-1} \nu_{s 1}\right) \gamma_{i 1} \\
\left.\frac{d^{2} p_{\gamma}}{d z^{2}}\right|_{z=1} & =\gamma_{s 2}+2\left(\frac{M-1}{\nu_{i 1}-1} \nu_{s 1}\right) \gamma_{s 1} \gamma_{i 1} \\
& +\left(\frac{M-1}{\nu_{i 1}-1} \nu_{s 1}\right)^{2} \gamma_{i 2} \\
\left.\frac{d^{3} p_{\gamma}}{d z^{3}}\right|_{z=1} & =\gamma_{s 3}+3\left(\frac{M-1}{\nu_{i 1}-1} \nu_{s 1}\right) \gamma_{s 2} \gamma_{i 1} \\
& +3\left(\frac{M-1}{\nu_{i 1}-1} \nu_{s 1}\right)^{2} \gamma_{s 1} \gamma_{i 2}+\left(\frac{M-1}{\nu_{i 1}-1} \nu_{s 1}\right)^{3} \gamma_{i 3}
\end{aligned}
$$

Now inserting the derivatives from Equations (159) and (165) into the Equations (147),(148) 
and (149) for $n_{1}, n_{2}$ and $n_{3}$, produces the factorial moments for combined gamma and neutron coincidences with multiplication.

$$
\begin{aligned}
n_{1} & =\underbrace{M \nu_{s 1}}_{\mathrm{n}}+\underbrace{\gamma_{s 1}+\left(\frac{M-1}{\nu_{i 1}-1} \nu_{s 1}\right) \gamma_{i 1}}_{\mathrm{g}} \\
n_{2} & =\underbrace{\gamma_{s 2}+2\left(\frac{M-1}{\nu_{i 1}-1} \nu_{s 1}\right) \gamma_{s 1} \gamma_{i 1}+\left(\frac{M-1}{\nu_{i 1}-1} \nu_{s 1}\right)^{2} \gamma_{i 2}}_{\mathrm{gg}} \\
& +\underbrace{2 M \nu_{s 1}\left[\gamma_{s 1}+\left(\frac{M-1}{\nu_{i 1}-1} \nu_{s 1}\right) \gamma_{i 1}\right]}_{\mathrm{gn}} \\
& +\underbrace{M^{2}\left[\nu_{s 2}+\left(\frac{M-1}{\nu_{i 1}-1}\right) \nu_{s 1} \nu_{i 2}\right]}_{\mathrm{nn}} \\
& +\underbrace{\gamma_{s 3}+3\left(\frac{M-1}{\nu_{i 1}-1} \nu_{s 1}\right) \gamma_{s 2} \gamma_{i 1}+3\left(\frac{M-1}{\nu_{i 1}-1} \nu_{s 1}\right)^{2} \gamma_{s 1} \gamma_{i 2}+\left(\frac{M}{\nu_{i 1}-1} \nu_{s 1}\right)^{3} \gamma_{i 3}}_{\mathrm{nnn}} \\
& +\underbrace{3 M \nu_{s 1}\left\{\gamma_{s 2}+2\left(\frac{M-1}{\nu_{i 1}-1} \nu_{s 1}\right) \gamma_{s 1} \gamma_{i 1}+\left(\frac{M-1}{\nu_{i 1}-1} \nu_{s 1}\right)^{2} \gamma_{i 2}\right\}}_{\mathrm{ggg}}
\end{aligned}
$$

\section{A.5 Effect of neutron capture on the neutron moments}

In the the previous derivation of the neutron moments with multiplication in Section A.3 as well as in the neutron multiplicity point Equations (6) from Section 2.2, it is assumed that the probability that a neutron is lost do to capture $p_{c}$ is small. If this probability is 
considered, the leakage multiplication becomes

$$
M=\frac{1-p-p_{c}}{1-k_{e f f}}
$$

$p \overline{\nu_{i}}$ is rewritten as $k_{e f f}$.

The probability generating function for the multiplication of a single neutron from Equation (151) must be rewritten to include $p_{c}$.

$$
h(z)=\left(1-p-p_{c}\right) z+p p_{\nu_{i}}[h(z)]
$$

The first three derivatives of $h(z)$ are now

$$
\begin{aligned}
\frac{d h(z)}{d z} & =\left(1-p-p_{c}\right)+p \frac{d p_{\nu_{i}}[h]}{d h} \frac{d h(z)}{d z} \\
\frac{d^{2} h(z)}{d z^{2}} & =p\left[\frac{d^{2} p_{\nu_{i}}[h]}{d h^{2}}\left(\frac{d h(z)}{d z}\right)^{2}+\frac{d p_{\nu_{i}}[h]}{d h} \frac{d^{2} h(z)}{\left.d z^{2}\right]}\right. \\
\frac{d^{3} h(z)}{d z^{3}} & =p\left[\frac{d^{3} p_{\nu_{i}}[h]}{d h^{3}}\left(\frac{d h(z)}{d z}\right)^{3}\right. \\
& +3 \frac{d^{2} p_{\nu_{i}}[h]}{d h^{2}} \frac{d h(z)}{d z} \frac{d^{2} h(z)}{d z^{2}}+\frac{d p_{\nu_{i}}[h]}{d h} \frac{d^{3} h(z)}{\left.d z^{3}\right]}
\end{aligned}
$$

The derivatives of $h(z)$ are now explicitly solved as before for later use.

$$
\begin{aligned}
& \left.\frac{d h(z)}{d z}\right|_{z=1}=M \\
& \left.\frac{d^{2} h(z)}{d z^{2}}\right|_{z=1}=M^{2} \frac{p}{\left(1-p \overline{\nu_{i}}\right)} \overline{\nu_{i}\left(\nu_{i}-1\right)} \\
& \left.\frac{d^{3} h(z)}{d z^{3}}\right|_{z=1}=M^{3} \frac{p}{\left(1-p \overline{\nu_{i}}\right)}\left[\overline{\nu_{i}\left(\nu_{i}-1\right)\left(\nu_{i}-2\right)}+3{\overline{\nu_{i}\left(\nu_{i}-1\right.}}^{2} \frac{p}{1-p \overline{\nu_{i}}}\right]
\end{aligned}
$$

The process of taking derivatives is now repeated with $\nu(z)=f_{s}[h(z)]$.

$$
\begin{aligned}
\frac{d \nu(z)}{d z} & =\frac{d f_{s}}{d h} \frac{d h}{d z} \\
\frac{d^{2} \nu(z)}{d z^{2}} & =\frac{d^{2} f_{s}}{d h^{2}}\left(\frac{d h}{d z}\right)^{2}+\frac{d f_{s}}{d h} \frac{d^{2} h}{d z^{2}} \\
\frac{d^{3} \nu(z)}{d z^{3}} & =\frac{d^{3} f_{s}}{d h^{3}}\left(\frac{d h}{d z}\right)^{3}+3 \frac{d^{2} f_{s}}{d h^{2}} \frac{d h}{d z} \frac{d^{2} h}{d z^{2}}+\frac{d f_{s}}{d h} \frac{d^{3} h}{d z^{3}}
\end{aligned}
$$

The equations are again solved for $z=1$. 


$$
\begin{aligned}
\left.\frac{d \nu(z)}{d z}\right|_{z=1} & =M \overline{\nu_{s}} \\
\left.\frac{d^{2} \nu(z)}{d z^{2}}\right|_{z=1} & =M^{2}\left[\overline{\nu_{s}\left(\nu_{s}-1\right)}+\frac{p \overline{\nu_{i}\left(\nu_{i}-1\right)}}{1-\nu_{i} p} \overline{\nu_{s}}\right] \\
\left.\frac{d^{3} \nu(z)}{d z^{3}}\right|_{z=1} & =M^{3}\left\{\overline{\nu_{s}\left(\nu_{s}-1\right)\left(\nu_{s}-2\right)}\right. \\
& +\frac{p}{1-\nu_{i} p}\left[3 \overline{\nu_{i}\left(\nu_{i}-1\right) \nu_{s}\left(\nu_{s}-1\right)}+\overline{\nu_{i}\left(\nu_{i}-1\right)\left(\nu_{i}-2\right)} \overline{\nu_{s}}\right] \\
& \left.+3\left(\frac{p}{1-\nu_{i} p}\right)^{2} \overline{\nu_{i}\left(\nu_{i}-1\right)} \bar{\nu}^{2}\right\}
\end{aligned}
$$

Equation (174) cannot be written entirely in terms of the leakage multiplication $M$ as was previously done in Equation (159). The other factor involving $p$ cannot be written in terms of the leakage multiplication alone because they do not contain $p_{c}$. Rather than treating $p$ as an unknown it may be more convenient to treat the additional unknown as the $k_{e f f}$ with the following substitution:

$$
\frac{p}{1-p \overline{\nu_{i}}}=\frac{k_{e f f}}{\overline{\nu_{i}}\left(1-k_{e f f}\right)}
$$

With this substitution and the substitution $\nu_{n}$ for the $n t h$ factorial moment Equation (174) becomes

$$
\begin{aligned}
\left.\frac{d \nu(z)}{d z}\right|_{z=1} & =M \nu_{s 1} \\
\left.\frac{d^{2} \nu(z)}{d z^{2}}\right|_{z=1} & =M^{2}\left[\nu_{s 2}+\left(\frac{k_{e f f}}{\overline{\nu_{i}}\left(1-k_{e f f}\right)}\right) \nu_{s 1} \nu_{i 2}\right] \\
\left.\frac{d^{3} \nu(z)}{d z^{3}}\right|_{z=1} & =M^{3}\left\{\nu_{s 3}+\left(\frac{k_{e f f}}{\overline{\nu_{i}}\left(1-k_{e f f}\right)}\right)\left[3 \nu_{s 2} \nu_{i 2}+\nu_{s 1} \nu_{i 3}\right]\right. \\
& \left.+3\left(\frac{k_{e f f}}{\overline{\nu_{i}}\left(1-k_{e f f}\right)}\right)^{2} \nu_{s 1} \nu_{i 2}^{2}\right\}
\end{aligned}
$$

In this form, the point equations will have two unknowns, leakage multiplication $M$ as well as the neutron multiplication constant $k_{e f f}$, rather than just one unknown. 


\section{APPENDIX B}

\section{FORMULAE}

\section{B.1 Discrete probability relationships}

General case

$$
P[A \cup B]=P[A]+P[B]-P[A \cap B]
$$

or

$$
P[A \cup B]=P[A]+P[B]-P[A] P[B]
$$

if $\mathrm{A}$ and $\mathrm{B}$ are independent.

Conditional probability

$$
P[A \mid B]=\frac{P[A \cap B]}{P[B]} .
$$

\section{B.2 Continuous probability relationships}

For the continuous case

$$
\begin{gathered}
f(x \mid y)=\frac{f(x, y)}{f_{Y}(y)} \\
f_{X}(x)=\int_{-\infty}^{\infty} f(x, y) d y=\int_{-\infty}^{\infty} f(x \mid y) f_{Y}(y) d y
\end{gathered}
$$

\section{B.3 Combinations, permutations and binomial expansion}

There are $\left(\begin{array}{l}n \\ k\end{array}\right)$ combinations of $n$ objects taken $k$ at a time:

$$
\left(\begin{array}{l}
n \\
k
\end{array}\right)=\frac{n !}{k !(n-k) !}
$$


Note that $\left(\begin{array}{l}n \\ k\end{array}\right)$ are the coefficients in the expansion

$$
\begin{aligned}
(x+y)^{n} & =\sum_{k=0}^{n}\left(\begin{array}{l}
n \\
k
\end{array}\right) x^{k} y^{n-k} \\
& =x^{n}+n x^{n-1} y+\frac{n(n-1)}{2 !} x^{n-2} y^{2}+\frac{n(n-1)(n-2)}{3 !} x^{n-3} y^{3}+\ldots
\end{aligned}
$$

The number of permutations of $n$ objects taken $k$ at a time:

$$
P(n, k)=k !\left(\begin{array}{l}
n \\
k
\end{array}\right)=\frac{n !}{(n-k) !}
$$

The probability of $k$ successes out of $n$ experiments with a probability of success being $p$ is

$$
P_{n}(k)=\left(\begin{array}{c}
n \\
k
\end{array}\right) p^{k}(1-p)^{n-k}=\frac{n !}{k !(n-k) !} p^{k}(1-p)^{n-k}
$$

\section{B.4 Discrete to continuous probability relationships}

Stirling's formula

$$
n !=n^{n} e^{-n} \sqrt{2 \pi n}
$$

DeMoivre-Laplace theorem: If $n p(1-p)<<1$ and $|k-n p| \approx<\sqrt{n p(1-p)}$ then

$$
P_{n}(k)=\left(\begin{array}{l}
n \\
k
\end{array}\right) p^{k}(1-p)^{n-k} \approx \frac{1}{\sqrt{2 \pi n p(1-p)}} e^{-\frac{k-n p}{2 n p(1-p)}}
$$

Poisson's theorem

$$
P_{n}(k)=\left(\begin{array}{l}
n \\
k
\end{array}\right) p^{k}(1-p)^{n-k} \approx \frac{(n p)^{k}}{k !} e^{-n p}
$$

\section{B.5 Stirling numbers of the second kind}

$$
S_{k}^{(n)}=\frac{1}{n !} \sum_{i=0}^{n}(-1)^{n-i}\left(\begin{array}{c}
n \\
i
\end{array}\right) i^{k}
$$

or recursively

$$
S_{k}^{(n)}=S_{k-1}^{(n-1)}+k S_{k}^{(n-1)}
$$

\section{B.6 Detector solid angle}

Cylindrical detector

$$
\Omega=2 \pi\left(1-\frac{d}{\sqrt{d^{2}+r^{2}}}\right)
$$


Rectangular detector[19]

$$
\begin{array}{r}
\Omega \\
=\tan ^{-1}\left(\frac{\left(x_{2}-x_{p}\right)\left(y_{2}-y_{p}\right)}{z_{p}\left[\left(x_{2}-x_{p}\right)^{2}+\left(y_{2}-y_{p}\right)^{2}+z_{p}^{2}\right]}\right) \\
-\tan ^{-1}\left(\frac{\left(x_{1}-x_{p}\right)\left(y_{2}-y_{p}\right)}{z_{p}\left[\left(x_{1}-x_{p}\right)^{2}+\left(y_{2}-y_{p}\right)^{2}+z_{p}^{2}\right]}\right) \\
-\tan ^{-1}\left(\frac{\left(x_{2}-x_{p}\right)\left(y_{1}-y_{p}\right)}{z_{p}\left[\left(x_{2}-x_{p}\right)^{2}+\left(y_{1}-y_{p}\right)^{2}+z_{p}^{2}\right]}\right) \\
+\tan ^{-1}\left(\frac{\left(x_{1}-x_{p}\right)\left(y_{1}-y_{p}\right)}{z_{p}\left[\left(x_{1}-x_{p}\right)^{2}+\left(y_{1}-y_{p}\right)^{2}+z_{p}^{2}\right]}\right)
\end{array}
$$




\section{REFERENCES}

[1] P. Baeten. Quantification of Transuranic Elements by Neutron Multiplicity Counting: A New Approach by Time Interval Analysis. PhD thesis, Vrije University of Brussel VUB, Brussel Belgium, April 1999.

[2] P. Baeten, M. Bruggeman, and R. Carchon. Single- and multi-deadtime parameter corrections of one- and two-dimensional Rossi-alpha distributions for time interval analysis in neutron coincidence counting. Nuclear Instruments and Methods in Physics Research, A 390:345-358, 1997.

[3] P. Baeten, M. Bruggeman, and R. Carchon. A generalized multiplication correction model for the description of fast and thermal neutron multiplication in neutron coincidence counting and time interval analysis. Nuclear Instruments and Methods in Physics Research, A 428:466-477, 1999.

[4] P. Baeten, M. Bruggeman, R. Carchon, and W. De Boeck. Neutron multiplicity measurements on $220 \mathrm{l}$ waste drums containing $\mathrm{Pu}$ in the range $0.1-1 \mathrm{~g}{ }^{240} P u_{\text {eff }}$ with the time interval analysis method. Nuclear Instruments and Methods in Physics Research, A 413:333-340, 1998.

[5] P. Baeten, M. Bruggeman, J. Paepen, and R. Carchon. Quantification of transuranic elements by time interval correlation spectroscopy of the detected neutrons. Applied Radiation and Isotopes, 52:399-402, 2000.

[6] K. Böhnel. The effect of multiplication on the quantitative determination of spontaneously fissioning isotopes by neutron correlation analysis. Nuclear Science and Engineering, 90:75-82, 1985.

[7] M. Bruggeman, P. Baeten, W. De Boeck, and R. Carchon. Neutron coincidence counting based on time interval analysis with one- and two-dimensional Rossi-alpha distributions: An application for passive neutron waste assay. Nuclear Instruments and Methods in Physics Research, A 382:511-518, 1996.

[8] G. S. Brunson. Multiplicity and correlated energy of gamma rays emitted in the spontaneous fission of Californium-252. Technical Report LA-9408-T, Los Alamos National Laboratory, June 1982.

[9] L. G. Chiang. Passive Coincidence Technique to Determine the Shape of Plutonium Objects Using Second Order Statistics. PhD thesis, Georgia Institute of Technology, March 2002.

[10] L. G. Chiang, M. Marseguerra, R. B. Oberer, S. A. Pozzi, and S. Ramoni. Utilization of neural networks and genetic algorithms to determine energy-threshold calibration curves in a safeguard experiment with fast neutrons and gammas. In IMORN2000, 2000. 
[11] L. G. Chiang and R. B. Oberer. Computation of detection efficiencies for NMIS fast plastic scintillators using a thick detector model. Technical Report ORNL/TM2000/316, Oak Ridge National Laboratory, November 2000.

[12] L. G. Chiang, R. B. Oberer, and S. A. Pozzi. Method to correlate CFD discriminator level and energy deposition by neutrons and photons in a fast plastic scintillating detector. Technical Report ORNL/TM-2000/193, Oak Ridge National Laboratory, 2000 .

[13] G. R. Cooper and C. D. McGillem. Probabilistic Methods of Signal and System Analysis. Harcourt-Brace-Jovanovich, 2 edition, 1986.

[14] S. Croft and P. B. Sharp. The measurement and calculation of the correlated neutron production rate from ${ }^{241} \mathrm{AmO}_{2} / \mathrm{Be}(\alpha, \mathrm{n})$ sources. Nuclear Instruments and Methods, A354:458-463, 1995.

[15] P. Desesquelles, A. J. Cole, A. Dauchy, A. Giorni, D. Heuer, A. Lleres, C. Morand, J. Saint-Martin, P. Stassi, J. B. Viano, B. Chambon, B. Cheynis, D. Drain, and C. Pastor. Cross talk and diaphony in neutron detectors. Nuclear Instruments and Methods in Physics Research, A307:366, 1991.

[16] N. Ensslin, W. C. Harker, M. S. Krick, D. G. Langner, M. M. Pickrell, and J. E. Stewart. Application guide to neutron multiplicity counting. Technical Report LA13422-M, Los Alamos National Laboratory, November 1998.

[17] E. P. Ficaro. KENO-NR: A Monte Carlo Code Simulating the ${ }^{252}$ Cf-source-driven Noise analysis Experimental Method for Determining Subcriticality. PhD thesis, University of Michigan, 1991.

[18] R. Ghetti, N. Colonna, and J. Helgesson. Influence of cross-talk rejection procedures on two-neutron intensity interferometry. Nuclear Instruments and Methods in Physics Research, A421:542, 1999.

[19] H. Gotoh and H. Yagi. Solid angle subtended by a rectangular slit. Nuclear Instruments and Methods, 96:485-486, 1971.

[20] T. Gozani. Active nondestructive assay of nuclear materials. Technical Report NUREG/CR-0602, US Nuclear Regulatory Commission, January 1981.

[21] W. Hage and D. M. Cifarelli. Correlation analysis with neutron count distributions in randomly or signal triggered time intervals for assay of special fissile materials. Nuclear Science and Engineering, 89:159, 1985.

[22] G. E. Hansen, H. H. Helmick, and J. D. Orndoff. Neutron counting statistics in basic fast critical asemblies. In Joint Japan-United States Seminar on Reactor Noise Analysis, 1968.

[23] N. W. Hill, J. T. Mihalczo, J. W. Allen, and M. M. Chiles. Optimization of nanosecond fission ion chambers for reactor physics applications. IEEE Transactions on Nuclear Science, NS-22:686, February 1975. 
[24] Glenn F. Knoll. Radiation Detection and Measurement, chapter 4. John Wiley \& Sons, Inc., 2nd. edition, 1989.

[25] M. S. Krick and J. E. Swansen. Neutron multiplicity and multiplication measurements. Nuclear Instruments and Methods in Physics Research, 219:384-393, 1984.

[26] A. V. Kuznetsov, I. D. Alkhazov, D. N. Vakhtin, V. G. Lyapin, V. A. Rubchenya, W. H. Trzaska, K. Loberg, and A. V. Daniel. Neutron multi-detector system: Mutual influence of its modules. Nuclear Instruments and Methods in Physics Research, A346:259-265, 1994.

[27] M. Marseguerra, E. Padovani, and S. A. Pozzi. MCNP-POLIMI: a Monte Carlo code for correlation measurements. Not yet published.

[28] M. Marseguerra, E. Padovani, and S.A. Pozzi. Simulating the wrong physics can yield correct results. In 3rd IMACS Seminar on Monte Carlo Methods MCM2001, September 2001.

[29] J. K. Mattingly. High Order Statistical Signatures from Source-Driven Measurements of Subcritical Fissile Systems. PhD thesis, University of Tennessee, 1998.

[30] J. K. Mattingly. Multivariate high order statistics of measurements of the temporal evolution of fission chain-reactions. Technical Report ORNL/TM-2001/45, Oak Ridge National Laboratory, 2001.

[31] J. K. Mattingly, J. T. Mihalczo, L. G. Chiang, and J. S. Neal. Preliminary analysis of joint RFNC-VNIEF/ORNL measurement performed in year 2000. Technical Report Y/LB-16,097, Y-12 National Security Complex, September 2001.

[32] J. K. Mattingly, J. T. Mihalczo, T. E. Valentine, and R.B. Perez. Reduction of background by higher order correlations with NMIS. In 41st Annual Meeting, New Orleans, Louisiana, July 16-20 2000. Institute for Nuclear Materials Management.

[33] J. T. Mihalczo. Use of ${ }^{252}$ Cf as a Randomly Pulsed Neutron Source for Prompt Neutron Decay Measurements. PhD thesis, University of Tennessee, 1970.

[34] J. T. Mihalczo. Comparison of source-sample fission coincidence method with the random driver method. Nuclear Methods of Analysis, 16:62, 1973.

[35] J. T. Mihalczo, J. A. Mullens, J. K. Mattingly, and T. E. Valentine. Physical description of Nuclear Materials Identification System (NMIS) signatures. Nuclear Instruments and Methods, 450:531-555, August 2000.

[36] J. T. Mihalczo, V. K. Paré, E. D. Blakeman, B. Damiano, R. B. Phillips, R. B. Bonner, D. B. Bopp, T. R. Chilcoat, J. DeClue, E. P. Elliott, G. D. Hackett, N. W. Hill, D. J. Nypaver, L. H. Thacker, W. T. Thomas, J. A. Williams, and R. E. Zumstein. NWIS signatures for confirmatory measurements with B33 trainers. Journal of Nuclear Materials Management, 25(3):64, 1997. 
[37] J. A. Mullens, J. K. Mattingly, T. E. Valentine, J. T. Mihalczo, and R. B. Perez. Incorporation of neutron and gamma multiplicity measurements in the ORNL Nuclear Materials Identification System (NMIS): Passive and active measurements. Technical Report Y/LB-15,984 R2, Oak Ridge Y-12 Plant, October 1998.

[38] T. Murakami, J. Kasagi, and H. Harada. Performance of a neutron multiplicity filter composed of six liquid scintillation detectors. Nuclear Instruments and Methods in Physics Research, A 241:172-176, 1985.

[39] R. Palit, H. C. Jain, P. K. Joshi, S. Nagaraj, H. V. Panchal, S. Bhattacharya, S. Chandra, S. K. Basu, and M. B. Chatterjee. Neutron detector array for measurement of neutron multiplicity. Nuclear Instruments and Methods in Physics Research, A 443:386-391, 2000.

[40] M. J. Paulus, J. T. Mihalczo, T. E. Valentine, J. A. Mullens, J. E. Breeding, T. Uckan, J. K. Mattingly, G. Turner, M. C. Smith, and J. A. McEvers. A novel method for determining pulse counting circuitry dead time using the Nuclear Weapons Inspection System. IEEE Transactions on Nuclear Science, 45(3):710, June 1998.

[41] R. B. Perez, J. L. Muñoz-Cobo, T. E. Valentine, J. T. Mihalczo, and J. A. Mullens. Incorporation of neutron and gamma multiplicity measurements in the ORNL Nuclear Materials Identification System (NMIS): Passive and active measurements. In INMM 1999, July 1999.

[42] J. Pluta, G. Bizard, P. Desesquelles, A. Dlugosz, O. Dorvaux, P. Duda, D. Durand, B. Erazmus, F. Hanappe, B. Jakobsson, C. Lebrun, F. R. Lecolley, R. Lednicky, P. Leszczynski, K. Mikhailov, K. Miller, B. Noren, T. Pawlak, M. Przewlocki, O. Skeppstedt, A. Stavinsky, L. Stuttge, B. Tamain, and K. Wosinska. Two-neutron interferometry measurements. Nuclear Instruments and Methods in Physics Research, A411:417, 1998.

[43] S. A. Pozzi, R. B. Oberer, and L. G. Chiang. The effect of neutron and gamma ray cross talk between plastic scintillating detectors. Nuclear Instruments and Methods in Physics Research, A481:739-748, 2002. Also ORNL/TM-2000/295.

[44] P. L. Reeder, A. J. Peurrung, R. R. Hansen, D. C. Stromwold, W. K. Hensley, and C. W. Hubbard. Detection of fast neutrons in a plastic scintillator using digital pulse processing to reject gammas. Nuclear Instruments and Methods, A422:84-88, 1999.

[45] T. D. Reilly, N. Ensslin, and H. A. Smith. Passive nondestructive assay of nuclear materials. Technical Report NUREG/CR-5550, US Nuclear Regulatory Commission, 1991.

[46] R. Sher and S. Untermyer II. The Detection of Fissionable Materials by Nondestructive Means. American Nuclear society, 1980.

[47] T. E. Valentine. MCNP-DSP: A Neutron and Gamma Ray Monte Carlo Calculation of Source Driven Noise-Measured Parameters. PhD thesis, University of Tennessee, 1995. 
[48] T. E. Valentine. MCNP-DSP: Monte Carlo N-Particle Transport Code System with Digital Signal Processing. Technical Report CCC-699, Oak Ridge National Laboratory, October 2000. Radiation Safety Information Computational Center.

[49] T. E. Valentine. Evaluation of prompt fission gamma rays for use in simulating nuclear safeguard measurements. Annals of Nuclear Energy, 28:191-201, 2001.

[50] T. E. Valentine, J. T. Mihalczo, R. C. Kryter, and V. C. Miller. Subcritical noise analysis measurements with fresh and spent research reactor fuel elements. Technical Report ORNL/TM-13752, Oak Ridge National Laboratory, February 1999.

[51] T. E. Valentine, R. B. Perez, J. A. Mullens, and J. T. Mihalczo. Passive multiplicity counting with the Nuclear Materials Identification System for plutonium. Technical Report P99-104067, Oak Ridge National Laboratory, July 1999.

[52] S. Y. Van der Werf. On the formalism of multiplicity measurements. Nuclear Instruments and Methods in Physics Research, 153:221-228, 1978.

[53] J. Wang, A. Galonsky, J. J. Kruse, P. D. Zecher, F. Deak, A. Horvath, A. Kiss, Z. Seres, K. Ieki, and Y. Iwata. Neutron cross-talk in a multi-detector system. Nuclear Instruments and Methods in Physics Research, A397:380, 1997.

[54] Z. Wang, Z. Xu, and S. Huang. Measuring time spectra with time-to-amplitude converters. Nuclear Instruments and Methods in Physics Research, A 450:415-418, 2000. 


\section{VITA}

Rick Oberer worked for the United States Air Force as an electronics engineer after receiving his B.S. degree in electrical engineering from the University of Dayton. He received his M.S. in physics while working on the fire control avionics for the F-16 aircraft at Ogden Logistics Center. He and his wife Lisa Chiang began course work in Nuclear and Radiological engineering in the Fall of 1994 through the distance-learning program, while in Utah. He continued this coursework after transferring to Robbins Air Force Base to work on the F-15 aircraft. They moved to Atlanta in the Fall of 1996 to finish the Ph.D. coursework. After two years in Atlanta, both Rick and Lisa took assignments at Oak Ridge National Laboratory for their Ph.D. research. Rick spent the first two years working on a solid state neutron detector for the Spallation Neutron Source with David Holcomb. After this he worked with John Mihalczo on the Nuclear Material Identification System which resulted in the dissertation, "Fission Multiplicity Detection with Temporal Gamma-Neutron Discrimination from Higher-Order Time Correlation Statistics." 